\title{
Multiagent Learning: dynamic games \& applications
}

Citation for published version (APA):

Hennes, D. (2013). Multiagent Learning: dynamic games \& applications. [Doctoral Thesis, Maastricht University]. Maastricht University. https://doi.org/10.26481/dis.20130516dh

Document status and date:

Published: 01/01/2013

DOI:

$10.26481 /$ dis.20130516dh

Document Version:

Publisher's PDF, also known as Version of record

\section{Please check the document version of this publication:}

- A submitted manuscript is the version of the article upon submission and before peer-review. There can be important differences between the submitted version and the official published version of record.

People interested in the research are advised to contact the author for the final version of the publication, or visit the DOI to the publisher's website.

- The final author version and the galley proof are versions of the publication after peer review.

- The final published version features the final layout of the paper including the volume, issue and page numbers.

Link to publication

\footnotetext{
General rights rights.

- You may freely distribute the URL identifying the publication in the public portal. please follow below link for the End User Agreement:

www.umlib.nl/taverne-license

Take down policy

If you believe that this document breaches copyright please contact us at:

repository@maastrichtuniversity.nl

providing details and we will investigate your claim.
}

Copyright and moral rights for the publications made accessible in the public portal are retained by the authors and/or other copyright owners and it is a condition of accessing publications that users recognise and abide by the legal requirements associated with these

- Users may download and print one copy of any publication from the public portal for the purpose of private study or research.

- You may not further distribute the material or use it for any profit-making activity or commercial gain

If the publication is distributed under the terms of Article $25 \mathrm{fa}$ of the Dutch Copyright Act, indicated by the "Taverne" license above, 
Daniel Hennes

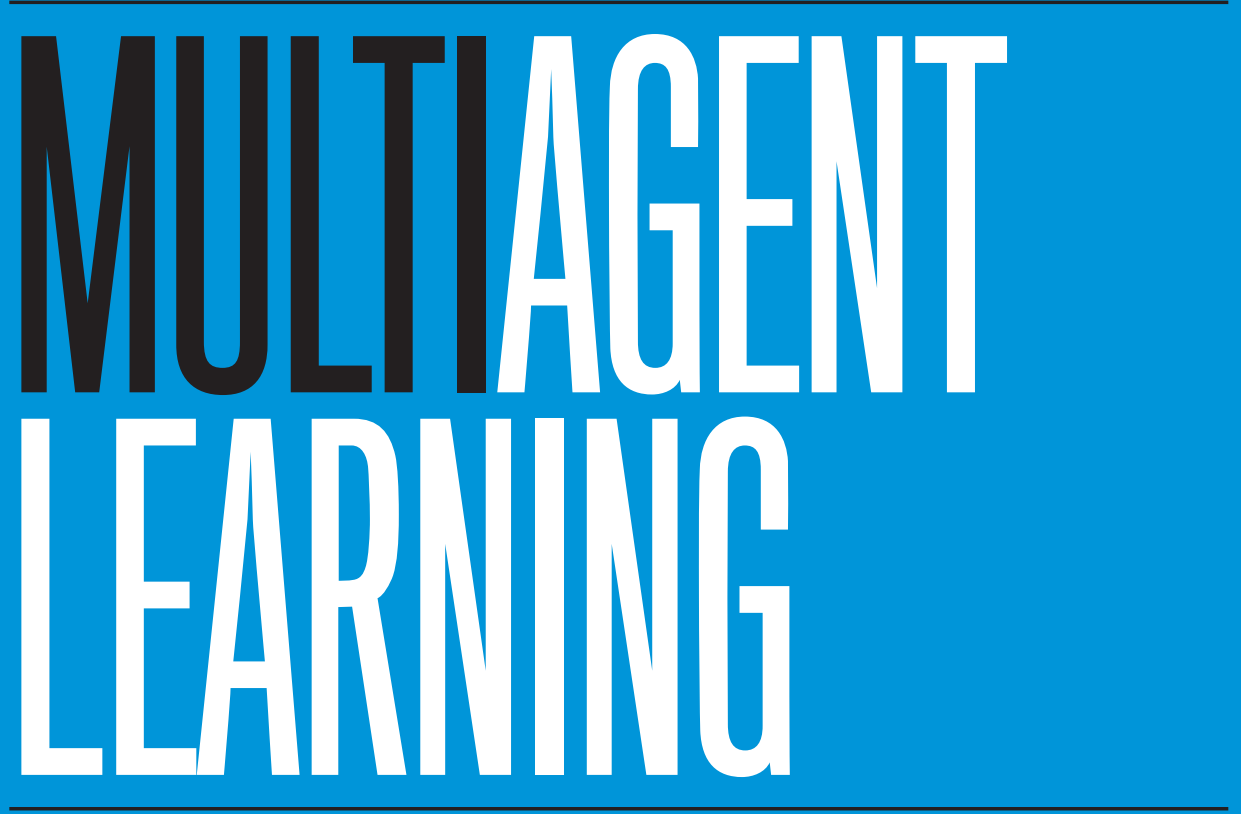

Dynamic Games \& Applications 


\section{Multiagent LeARNiNG \\ Dynamic Games \& Applications}




\title{
Multiagent LeARNiNG \\ Dynamic Games \& Applications
}

\author{
DISSERTATION
}

to obtain the degree of Doctor at

Maastricht University,

on the authority of the Rector Magnificus,

Prof. dr. L. L. G. Soete,

in accordance with the decision

of the Board of Deans,

to be defended in public

on Thursday May I6, 2013 at 14:00 hours

by

Daniel Hennes 


\section{Supervisor:}

Prof. dr. G. Weiss

\section{Co-supervisors:}

Dr. K. P. Tuyls

Prof. dr. K. Tumer (Oregon State University)

\section{Assessment Committee:}

Prof. dr. ir. R. L. M. Peeters (chairman)

Prof. dr. R. Babuska (Delf University of Technology)

Dr. ir. K. Driessens

Prof. dr. ir. J. C. Scholtes

Prof. dr. M. Tambe (University of Southern California)

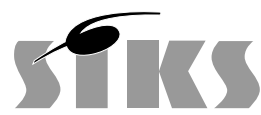

SIKS Dissertation Series No. 2013-15

The research reported in this thesis has been carried out under the auspices of SIKS, the Dutch Research School for Information and Knowledge Systems.

Printed by Optima Grafische Communicatie, Rotterdam, The Netherlands.

ISBN 978-94-6169-389-I

(C) Daniel Hennes, 2013.

All rights reserved. No part of this publication may be reproduced, stored in a retrieval system, or transmitted, in any form or by any means, electronically, mechanically, photocopying, recording or otherwise, without prior permission of the author. 


\section{Contents}

\section{Contents $\diamond \mathrm{i}$}

\section{Introduction $\diamond \mathrm{I}$}

I.I Games, Equilibria and Evolution $\diamond \quad 2$

I.2 The Need for Multiagent Learning $\diamond 7$

I.3 Problem Statement and Research Questions $\diamond 9$

I.4 Related Fields $\diamond$ I2

I.5 Contributions and Dissertation Outline $\diamond \mathrm{I} 3$

\section{Part I: Formal Framework $\diamond$ I5}

2 Fundamentals of Multiagent Learning $\diamond \mathrm{I} 7$

2.I Markov Decision Processes $\diamond \mathrm{I} 8$

2.2 Value Iteration and Policy Iteration $\diamond 20$

2.3 Q-Learning $\diamond 2 \mathrm{I}$

2.4 Learning Automata $\diamond 24$

2.5 Stochastic Games $\diamond 26$

2.6 Multiagent Learning Algorithms $\diamond 27$

2.7 Summary $\diamond 35$

3 Evolutionary Models of Learning $\diamond 37$

3.I Evolutionary Game Theory $\diamond 38$

3.2 Replicator Dynamics $\diamond 40$

3.3 Learning Dynamics $\diamond 44$

3.4 Dynamics of a Population of Learners $\diamond 53$

3.5 Summary $\diamond 55$

4 Stochastic Game Dynamics $\diamond 57$

4.I Piecewise Replicator Dynamics $\diamond 58$

4.2 State-Coupled Replicators Dynamics $\diamond 68$

4.3 Symmetric State-Coupled Replicator Dynamics $\diamond 74$

4.4 State-Coupled Replicator Dynamics with Mutation $\diamond 76$

4.5 Summary $\diamond 8 \mathrm{I}$ 


\section{Part II: Applications $\diamond 83$}

5 Take-it or Leave-it Negotiation $\diamond 85$

5.I Colored Trails $\diamond 87$

5.2 Proposer Metastrategies $\diamond 89$

5.3 Generalizing the Analysis $\diamond 96$

5.4 Empirical Evaluation $\diamond 98$

5.5 Evolutionary Analysis $\diamond \mathrm{IO2}$

5.6 Discussion $\diamond$ IO3

5.7 Summary $\diamond$ IO4

6 The Value of Information $\diamond$ IO5

6.I Auctions and the Value of Information $\diamond$ Io6

6.2 Market Model $\diamond$ Io8

6.3 Evolutionary Analysis $\diamond$ II4

6.4 Summary $\diamond$ II7

7 Collision Avoidance $\diamond$ II9

7.I The Game of Chicken $\diamond$ I2O

7.2 Velocity Obstacles $\diamond$ I2I

7.3 Evolutionary Analysis $\diamond$ I24

7.4 Summary $\diamond \mathrm{I} 28$

8 Conclusion $\diamond \mathrm{I} 29$

8.I Contributions and Answers to the Research Questions $\diamond \quad$ I29

8.2 Limitations and Future Research Perspectives $\diamond \mathrm{I} 32$

References $\diamond \mathrm{I} 35$

Publications $\diamond \mathrm{I} 5 \mathrm{I}$

List of Figures $\diamond \mathrm{I} 55$

List of Tables $\diamond$ I57

Summary $\diamond \mathrm{I} 59$

Samenvatting $\diamond \mathrm{I} 6 \mathrm{I}$

Acknowledgements $\diamond \mathrm{I}_{3}$

SIKS Dissertation Series $\diamond \mathrm{I}_{6} 5$ 


\section{1 \\ Introduction}

Game theory has a great influence on computer science, most notably on the branches of artificial intelligence and theoretical computer science. Shoham (2008) names multiagent learning as one of most the fruitful interaction grounds between computer science and game theory. The study of learning has a long and rich history in both game theory (Fudenberg and Levine, 1998) and artificial intelligence (Mitchell, I997). In traditional game theory, equilibrium play results from deliberative reasoning of players under a strict rationality assumption and with common knowledge of the structure of the game, i.e., payoffs. Interactive learning is the study of how equilibria arise as the long-run outcome of a process in which players under bounded rationality seek optimality. Machine learning, as one of the core fields of artificial intelligence, is concerned with the computational aspects of learning in natural as well as technical single- and multiagent systems (Sen and Weiss, 1999). In particular, reinforcement learning is a machine learning technique by which agents learn from repeated interactions with the environment. The crux of multiagent learning is to understand the dynamics of interactions and mutual adaptation. The inherent complexity of any multiagent learning process is due to a moving target: the learning process of one agent causes a change in the behavior of other agents, causing the first agent to keep adapting too - and thus closing the circle. This dissertation is contributing to both the theory of learning in games, as well as multiagent reinforcement learning.

This chapter introduces games, equilibria and population dynamics. We argue why adaptation is inevitable in multiagent systems and hereupon discuss learning algorithms that fulfill this need. Furthermore, the strengths and benefits of an evolutionary game theoretic approach to multiagent learning are emphasized. The problem statement and objective of this dissertation are formulated, as well as a number of research questions that have guided this work. Finally, this chapter is concluded by an overview of related fields, a brief review of the main contributions, and an outline of this dissertation. 


\section{I.I Games, Equilibria and Evolution}

Game theory studies strategic interactions where multiple players act rationally in order to maximize their expected payoffs. The origin of modern game theory dates back to the seminal work by von Neumann and Morgenstern (I944). Game theory has since found applications in very diverse fields. Players can represent companies in the realm of economics, single individuals in the psychological or social science domain, animals, bacteria, or plants in the field of biology, as well as computer agents situated in a multiagent or multi-robot setting.

To provide an intuitive understanding of the methodology of game theory we shall first consider a particular strategic encounter. The prisoners' dilemma (Axelrod, I984) is a prevalent example to showcase game theoretical decision making and analysis. We first introduce the classical predicament, while gradually extending the problem to repeated interaction, and evolution.

Example I (Prisoners' dilemma). The dilemma presents itself in the following situation. Two subjects are under arrest. The police has not collected enough evidence to provide the prosecution with grounds for a severe accusation. Thus the two prisoners are separated for interrogation and are both confronted with the same choice: either confess the crime and hence "defect" and betray the other suspect, or to deny any accusation and hence "cooperate" with the other suspect. If one suspect testifies while the other remains silent, the defector is released while his loyal fellow is convicted to serve the full sentence. If both defect, both have to serve a long sentence, only slightly reduced for cooperating with the police. If both deny all accusations, they serve a short sentence for a minor charge. How should the prisoners choose rationally in order to minimize penalty?

Seemingly, the best option is to cooperate, both parties only serve a short sentence and are set free after a relatively short time. However, this intuition suggests a nonrational decision. Game theory provides the tools to reason about such a strategic situation and identify the rational choice. Let us consider the options of a suspect under the following set of assumptions. A suspect is self-interested and therefore only cares about his own well-being (or degree of punishment in our example) and thus does not profit from lowering the sentence of the other. Furthermore, any communication between suspects is inhibited and both are ensured that the other is provided with the same information and faces the same choice. Both have the same option to choose exactly one of the two strategies "defect" or "cooperate" and the outcome of the game is independent of the order or identity of the players. In other words, the game is symmetric. Since no communication is allowed we might as well assume that players act simultaneously.

Suppose a player chooses to "cooperate". If the other one cooperates as well the player is convicted to serve a short sentence; if the other one betrays him he serves the 
Table I.I: Payoff table of the prisoners' dilemma. The first value in each cell represents the payoff to the row player and the second value represents the payoff to the column player.

\begin{tabular}{c|c|c|}
\multicolumn{1}{c}{} & \multicolumn{1}{c}{ Cooperate } & Defect \\
\cline { 2 - 3 } Cooperate & 3,3 & 0,5 \\
\cline { 2 - 3 } Defect & 5,0 & 1,1 \\
\cline { 2 - 3 } & &
\end{tabular}

full sentence. If the first player instead choses to "defect", he is set free in the former case while as well serving the full sentence in the later case. We observe that cooperating is dominated by defecting, i.e. for each adversarial response, "defect" yields as least as much payoff as "cooperate". Thus classical game theoretic analysis concludes that players make the rational decision to mutually defect and hence end up serving both the maximum sentence. While intuition suggests otherwise, in the light of rationality the suspects face an unwinnable situation.

\section{Normal Form Games}

In accordance with von Neumann and Morgenstern (1944), we represent an $n$-player game by an $n$-dimensional matrix. Each matrix entry is an $n$-tuple determining the payoff for each player and there is one entry for each possible combination of strategies. Such a matrix game is also called a normal form game or a game in strategic form. The behavior of a player can be captured by a probability distribution over all strategies. If the probability mass function of said distribution equals 1 for one of the strategies, the player follows a pure strategy otherwise a mixed strategy. The n-tuple consisting of all strategies is called a strategy profile.

The normal form representation of our example, the prisoners' dilemma, is shown in Table I.I. If both players serve the full sentence the payoff for each player is 1 ; if one suspect betrays the other the payoffs are 5 and 0 respectively; while being convicted only for the minor felony results in a payoff of 3 . Note that the matrix assigns numerical payoffs that describe the preference relation for players over outcomes of the game. Only the preference relation is important, not the exact numerical values. We could just as well define these payoffs as the negative of years served on a certain sentence.

\section{Solution Concepts}

For two-player fully antagonistic games, the gain of one player becomes the other's loss. That is to say that each 2-tuple in the payoff matrix sums up to zero (alternatively to any constant) and the game is hence called zero-sum (or constant-sum). Consider 
Table I.2: Payoff table of the matching pennies game. Values represent the payoff to the row player; loss of the column player.

\begin{tabular}{c|c|c|}
\multicolumn{1}{c}{} & \multicolumn{1}{c}{ Heads } & \multicolumn{1}{c}{ Tails } \\
\cline { 2 - 3 } Heads & 1 & -1 \\
\cline { 2 - 3 } Tails & -1 & 1 \\
\cline { 2 - 3 } & &
\end{tabular}

the payoff matrix shown in Table I.2, where entries show only the payoff to the row player and thus the loss of the column player.

Example 2 (Matching pennies). Both players choose for one side of a penny and reveal their choice simultaneously. If both pennies show the same face, the row player keeps the coins; for a mismatch the column player gets rewarded.

Von Neumann's work contains a proof that each zero-sum game has a value. The row player can guarantee this value against any play of the opponent. Accordingly, the column player can guarantee a maximum loss of this value. We can thus compute the value of a zero-sum game as the minimum payoff the row player can guarantee if the column player maximizes reward, i.e. the minimax value (von Neumann, I928). The value of matching pennies is 0 , by playing a mixed strategy of probability $\frac{1}{2}$ "heads" and $\frac{1}{2}$ "tails" the row player can guarantee an expected payoff of 0 . However, our original example, the prisoners' dilemma, is clearly not zero-sum. We call this a coopetitive encounter, featuring flavors of cooperation and competition, a general-sum game. What is the solution to such a game?

In general, game theory is concerned with identifying so called equilibrium solutions in which each player is committed to a certain strategy guided by rational selfinterest. In his fundamental work, Nash (1950, I95I) studies non-cooperative games and devises a solution concept that would become the renowned Nash equilibrium. A Nash equilibrium is a strategy profile for which no player can improve her payoff by unilaterally deviating from her strategy. Players play a profile of mutual best responses in equilibrium. Nash's existence theorem (1950) states that any normal form game with a finite number of players and finitely many actions has at least one Nash equilibrium. In the prisoners' dilemma, strategy "cooperate" is dominated by strategy "defect". This means that "defect" yields a higher payoff, no matter which strategy the other player adopts. Hence, the Nash equilibrium is located in the bottom-right corner of Table I.I, corresponding to both players defecting.

We have arrived at the Nash solution by purely rational reasoning. However, this is an unfavorable outcome for the two suspects. If for example communication between the players were allowed and trust would not play a role, mutual commitment 
to "cooperate" would clearly lead to a more desirable outcome. In cooperative settings, Pareto optimal or Pareto efficient solutions are preferred over Nash's solution concept. A Pareto optimal solution implies that no player can improve his expected payoff without making at least one other player worse off. Note that Pareto efficiency does not require players to change their actions unilaterally, they may as well coordinate their action in order to increase payoff. In the prisoners' dilemma game, mutual cooperation is Pareto efficient. However, if only one player cooperates the joint strategy is also Pareto efficient: the cooperative player has to switch to defecting in order to improve his payoff, which of course makes the other player worse off.

Classical game theory postulates that the analyzed game is played once by rational players who are fully informed about the particular strategic situation they are facing, in particular each other's preferences over outcomes. The players' rationality is unbounded, meaning not limited by time, information or computing constraints. Solution concepts, like the Nash equilibrium, offer predictions on the outcome of games, however, they do not state how players arrive at an equilibrium. For a more detailed account of classical game theory, the interested reader is advised to consult the books by Fudenberg and Tirole (1991), Gibbons (1992), or Osborne and Rubinstein (1994).

\section{Evolutionary Game Theory}

Nash (I950, I95I) provided a powerful tool in formalizing the solution concept that bears his name. Small games, like the ones presented in Example I and 2, have only one Nash equilibrium solution and this equilibrium offers a unique prediction of the result of the rational thought process of both players. In general, more complex games have typically many equilibria and thus give rise to the question of which equilibrium one should select in order to predict the rational outcome of the game. Further refining the equilibrium criteria allows to discard certain Nash equilibria and thus seems like a viable option to solve this equilibrium selection problem. Many different refinements were proposed, each following an alternative take on the definition of rationality (Govindan and Wilson, 2008). This vast variety of interpretations soon lessened the usefulness of equilibrium refinements. The seminal work by Maynard Smith (1974) on The Theory of Games and the Evolution of Animal Conflicts has added a new perspective to game theory: game theory predicts the behavioral patterns of animals - animals with very limited cognitive abilities, e.g., birds and insects. In addition, behavioral economics has shown that also humans do not always act rationally and are often unable to estimate or calculate likely costs and benefits of their actions, e.g., see Kahneman et al. (I982). Evolutionary game theory relaxes the assumption of hyper rationality and argues that equilibrium play is the mere result of an evolutionary process guided by trial and error. 
Let us reconsider our initial example, the prisoners' dilemma, in order to illustrate the application of an evolutionary process to games. Instead of playing the game just once, the interaction is assumed to be repeated over multiple stages. In the iterated prisoners' dilemma the two suspects encounter the same predicament over and over again. Clearly, this can affect the equilibrium strategies. If the game is repeated infinitely often, rational players can obtain the Pareto efficient reward by invariably cooperating. If however the game is played $N$ times and this information is known to the players, the rational strategy is to always defect which can be shown by backwards induction (Gintis, 2000).

Axelrod and Hamilton (I98I) have proposed a tournament version of the iterated prisoners' dilemma. Each player has to choose a strategy, possibly depending on memory of previous encounters. Interactions between pairs of individuals occur on a probabilistic basis while natural selection favors individuals that have performed better in the past. The derived model as well as results of the tournament show how cooperation based on reciprocity can evolve in a mechanism that advocates self-interest. The simplest deterministic strategy, called tit-for-tat, has won the tournament. Tit-for-tat starts by cooperating and hereafter responds by playing the action the opponent has played in the last stage.

Population games provide a simple and general framework to study the dynamics of strategy changes in large populations where individuals are randomly matched to play a normal form game. In particular, the success of a strategy depends on biologicalinspired genetic operators such as natural selection and mutation. A population is formed by a finite or infinite number of individuals each of whom is associated with a pure strategy. Two randomly matched individuals play a normal form game according to their pure strategies while their payoff determines the evolutionary success of the represented strategies.

The core solution concept of evolutionary game theory is evolutionary stability. We call a strategy an evolutionarily stable strategy (Maynard Smith, I974) if it cannot be invaded by a mutant strategy. Initially the entire population plays a particular mixed strategy. At some moment in time a mutation occurs and the mutant strategy is played by a small fraction of the population. The mutant strategy might invade the population, thus growing until it overtakes the entire population or it might go extinct due to natural selection.

Maynard Smith only defines a static solution concept that does not model the dynamic evolution of the population explicitly. Taylor and Jonker (I978) have introduced the replicator dynamics to provide a dynamic model of evolution based on ordinary differential equations. The replicator dynamics describe the deterministic evolutionary dynamics of population games. Although initially biologically inspired, replicator dynamics also find application in economics (Weibull, I997). A general introduction 
as well as more detailed treatments of evolutionary game theory can be found in the excellent books by Hofbauer and Sigmund (1998), Weibull (I995), and Sandholm (201I).

\section{I.2 The Need for Multiagent Learning}

Learning in multiagent systems gains a lot of interest in the international research community (Hoen et al., 2006; Shoham et al., 2007; Vohra and Wellman, 2007; Stone, 2007; Tuyls and Parsons, 2007; Buşoniu et al., 2008; Tuyls and Weiss, 2012). Multiagent systems is a research area of artificial intelligence that studies the decentralized interactive behaviors of autonomous artificial agents. Multiagent systems offer a novel solution paradigm that can even cope with complex problems of today's society. More and more contemporary technological challenges require decentralized solutions. Examples include, but are not limited to, the control of multiple autonomous vehicles for exploration, surveillance or rescue missions, distributed sensing, small interconnected smart devices as well as automated control systems for data, urban or air traffic (Tumer and Agogino, 2007). Multiagent systems represent an effective framework to study the interactions of agents and furthermore to develop solutions to control and improve such systems. This strong focus on agent interactions distinguishes the multiagent approach from other disciplines like distributed control or distributed artificial intelligence.

The demand for multiagent systems is widespread and originates from a collection of significant advantages. Multiagent systems have built-in redundancies that allow for robustness; if one or a few components fail the system is still able to operate properly. In addition, different tasks can be assigned to independent agents pursuing these goals in parallel. Multiagent systems are modular per definition and components can be easily added or removed, and as such also scale better than centralized systems (Weiss, I999). Furthermore, multiagent systems reflect nature more precisely. In reality, agents are usually not isolated. They need to interact with each other and quickly adapt to changing environments. These qualities make multiagent systems desirable or even essential for a variety of applications.

While a multiagent approach offers an elegant solution paradigm to issues like fault tolerance and load balancing, it also introduces new challenges. The presence of multiple agents results in a highly dynamic and nondeterministic environment. Clearly, it is hardly possible to derive a straight forward mapping for each agent that transforms observed input to actions. Interactions between agents and with the environment inevitably cause very complex systems which are not trivial to control. This prohibits the possibility to directly engineer intelligent behavior. However, if adaptation is used on an individual level it often leads to the emergence of intelligent behavior of the system as a whole (Brooks, I99I). In order to facilitate adaptation to changes in the environment and other agents, learning is crucial. 


\section{Reinforcement Learning}

The learning process of an adaptive agent within a multiagent system can be modeled as a reinforcement learning task. The agent iteratively interacts with its environment and other agents while observing feedback from the environment. In order to maximize expected long term reward, the action behavior is adapted according to this reinforcement signal. As opposed to supervised learning the agent is not provided with the correct or optimal state-action pair at any time. For a general introduction to reinforcement learning, consult the book by Sutton and Barto (I998) or for a concise overview the review paper by Thrun and Littman (2000).

Reinforcement learning techniques are subdivided in value iteration and policy iteration. Value based learners estimate a state-action value function that determines the utility of performing a given action in a given state. Hereafter, this value function is used to derive a policy that describes the behavior of the agent. Contrary to the value based approach, policy iterators learn directly in the policy space. Traditionally, reinforcement learning is strongly associated with the notion of value iteration, e.g. Q-learning (Watkins, I989). The learning automaton (Narendra and Thathachar, I974) is an example of a simple reinforcement learning algorithm following the policy iteration scheme.

The learning performance of contemporary reinforcement learning techniques has been studied in great depth experimentally and formally for a diversity of single agent control tasks (Kaelbling et al., I996; Sutton and Barto, I998). Assuming sufficient learning cycles and a stationary environment convergence to the optimal policy is guaranteed. However, these results do not hold for complex multiagent environments (Shoham et al., 2007). Learning in multiagent systems is inherently more challenging than singleagent learning. Several agents interact with the environment and other agents; in addition their behavior might alter by self-adaptation which complicates any reasonable prediction. Thus, with the presence of multiple adaptive agents the environment becomes highly dynamic and non-deterministic. Although multiagent learning is far more complex and conditions differ, single-agent reinforcement learning algorithms have been applied in multiagent settings with respectable results (Thathachar and Phansalkar, I995; Peeters, 2003). This naïve extension to multiagent setting is henceforth called independent multiagent reinforcement learning. Independent learners mutually ignore each other and only implicitly perceive the interaction with other agents as noise in a stochastic environment.

\section{Joint Action and Equilibrium Learners}

In addition to the naïve approach of independent learners, mostly value-iteration based reinforcement learning techniques have been extended to the multiagent case. Joint 
action learners (Claus and Boutilier, I998) estimate the value of playing a certain joint action rather than independent state-action pairs. This approach allows to represent the decision problem with a lower level of abstraction, however, it requires that the joint action is observable. In addition to joint action value estimates, learners track the empirical frequencies of joint action play. This "opponent model" allows to devise an optimal policy under current value estimates and the assumption that all other agents play stationary policies.

If besides the joint action also the payoffs to all players are observable, joint action values can be tracked for all players and represent a normal form game. Equilibrium learners make use of a specific game theoretic solution concept to predict which joint action is going to be played in the long-run and use this to estimate the value of the state. Nash-Q learning (Hu and Wellman, 2003) and minimax-Q (Littman, I994) are well known examples of equilibrium learners, using the Nash equilibrium concept and the value of zero-sum games respectively.

\section{I.3 Problem Statement and Research Questions}

Many sophisticated multiagent learning algorithms, for instance equilibrium learners (Littman, I994, 200I; Hu and Wellman, 2003; Greenwald and Hall, 2003), have been studied in great detail. Most of these studies focus on simple, abstracted environments often modeled by games. The structure of the game is either known to all agents a priori or immediate payoffs are fully observable. While these algorithms show promising results, applications in real-life domains are lacking. Especially in competitive domains, full payoff observability and thus the preferences over outcomes of other agents, is not a realistic assumption. For most realized adaptive multiagent systems, simple independent learners are employed, i.e., single-agent learning in a multiagent system. Although not nearly as sophisticated, and in principle ignorant to the presence of other agents, this approach has been shown to be successful despite the fact that guarantees of convergence do no longer hold.

The aim of this dissertation is to contribute to the understanding of the complex dynamics of independent reinforcement learners in multiagent settings. Börgers and Sarin (1997) have derived a formal relation between independent multiagent reinforcement learning and the replicator dynamics. This relation between replicators and reinforcement learning has been extended to different algorithms such as learning automata and Q-learning (Tuyls et al., 2003b; Tuyls, 2004). Exploiting the link between reinforcement learning and evolutionary game theory is beneficial for a number of reasons. The majority of contemporary reinforcement learning algorithms are blackbox models. This makes it difficult to gain detailed insight into the learning process and parameter tuning becomes a cumbersome task. Analyzing the learning dynamics 
helps to determine parameter configurations before learners are actually deployed in the task domain. Furthermore, the possibility to formally analyze multiagent learning algorithms helps to derive and compare new algorithms (Tuyls et al., 2003a; Panait et al., 2008; Kaisers and Tuyls, 20Io). However, the approach has been limited to simple, abstract, two-player games. Considering the strengths and benefits but also the limitations of this approach we can formulate the following problem statement:

Problem statement: Population games, and in particular the replicator dynamics, provide the tools and a formal framework to study the dynamics of reinforcement learning in multiagent systems. The two main limitations of this approach are: (a) the restriction to stateless repeated two-player games and $(b)$ the limited application to complex real world problems.

In order to approach this general problem, we refine said problem statement in six research questions. Question I through 3 address the problem of advancing the theoretical framework of evolutionary models of learning; question 4 through 6 address the practical application to complex real world problems.

The majority of the literature on replicator dynamics considers two-player games: symmetric games with one population of individuals or asymmetric games with two co-evolving populations of individuals. The later case relates to the situation of two learning agents, each represented by a population. Although the asymmetric or multipopulation replicator dynamics can be generalized to games with more than two players, the resulting dynamics are difficult to analyze or visualize and thus loose much of their explanatory power. If the number of agents (populations) is increased to hundreds or thousands, the resulting system becomes simply intractable. Symmetric "one population" replicator dynamics model random interactions of two individuals, where the interaction is defined by the underlying game. This model is very suitable for most multiagent systems, e.g.: negotiations or auctions, where random and anonymous pairwise interactions between buyers and sellers occur frequently. However, the replicator model assumes fixed phenotypes, i.e., each individual within a population plays a predetermined strategy that must not be adopted over time. This leads to the first research question which is answered in Section 3.4:

Question I: What is the multiagent learning interpretation of a single population under the symmetric continuous time replicator dynamics?

Furthermore, as the replicator dynamics are limited to the analysis of single-state normal form games with symmetric or asymmetric payoff structures, so is the derived approach to model multiagent learning dynamics. Although some real world problems might be modeled by single-state game abstractions, the majority of problems naturally relates to multi-state situations. Research question 2 and 3 investigate to 
what extent the evolutionary game theoretic approach to multiagent learning can be extended to multi-state (stochastic) games. Both questions are answered in Chapter 4.

Question 2: To what extent does the relation between asymmetric continuous time replicator dynamics and individual reinforcement learning generalize to stochastic games?

Question 3: What is the stochastic game generalization of the selection-mutation model of Q-learning by Tuyls et al. (2003b)?

In addition to the extension of the formal framework of evolutionary models of reinforcement learning, we also investigate the application of this approach to complex real world problems. For many such problems the full game-theoretic analysis is intractable. Walsh et al. (2002) have developed a method to analyze exogenously specified heuristic strategies, rather than atomic actions. The methodology allows to compute expected payoffs to agents as a function of the joint strategy space, identify equilibria, and analyze the evolutionary dynamics. The research guided by question 3 through 6 applies a similar methodology to three real world application domains: bargaining, markets, and collision avoidance.

Question 4 studies multiagent bargaining settings which feature multiple players, large action spaces, and a relationship among players' goals, tasks and resources. We show how to reduce such interactions to a set of bilateral normal form games in which the strategy space is significantly smaller than the original setting. Research question 4 is answered in Chapter 5 .

Question 4: How can the evolutionary model of learning be applied to complex real-world problems such as multiagent bargaining situations?

Question 5 investigates the competitive advantage of price signal information for traders in continuous double auctions. Previous work has established that more information about the future price development does not guarantee higher returns. We investigate if this finding also holds for a large population of traders and how the dynamics are influenced when information comes at a cost. Question 5 is answered in Chapter 6.

Question 5: What is the value of future price signal information; and in particular: is there an evolutionary advantage of foresight in markets?

The final research question studies the evolutionary stability of various collision avoidance methods all following the velocity obstacle paradigm (Fiorini and Shiller, I998). Collision avoidance is crucial to everyday car, air, and vessel traffic as well as crowd dynamics in evacuation scenarios. Question 6 is answered in Chapter 7.

Question 6: What are the evolutionary dynamics of collision avoidance; and in particular, is reciprocity in collision avoidance an evolutionarily stable strategy? 


\section{I.4 Related Fields}

This section briefly discusses the related fields we consider most relevant to multiagent reinforcement learning - in particular, empirical game theory, algorithmic game theory, and optimal adaptive control.

\section{Empirical Game Theory}

Empirical game theory (Walsh et al., 2002) combines simulation and traditional game theoretic tools to estimate a model of complex strategic interactions. The typical game studied in classical game theoretic literature is an abstract, very stylized strategic encounter. Often, the goal is to study a game that is representative of a wide variety of situations and thus allows us to draw general conclusions about strategic reasoning. The prisoners' dilemma is not studied to improve law enforcement procedures in particular, rather to show how self-interest hampers cooperation and leads to Pareto suboptimal outcomes. In addition to the study of general phenomena, one might be interested in a specific interaction. Although in theory possible, modeling complex multiagent systems quickly results in intractable game models. Often the state- and strategy-spaces are large and thus pose limits on the computability of equilibrium solutions. A popular approach is to abstract the strategy space to a small number of metastrategies. However, one of the toughest challenges in game theory is to set meaningful payoff values. Empirical game theory provides the tools to estimate these payoff values from simulation or real data in a variety of domains. Walsh et al. (2002) estimate heuristic payoff tables for a continuous double auction and a dynamic pricing game through simulation. Kiekintveld et al. (2006) use empirical game theoretic methods to analyze an ancient variant of chess with a particularly large strategy space. Ponsen et al. (2009) use a heuristic payoff table to analyze a large number of real world poker games. Empirical methods are often indispensable when analyzing learning in complex domains. In particular, the work presented in Part II of this dissertation makes use of such tools.

\section{Algorithmic Game Theory}

Like multiagent learning, algorithmic game theory (Nisan et al., 2007) is a field situated at the crossroad of computer science and game theory. It is primarily concerned with the computational aspects of solving games, i.e., the complexity of computing equilibria, but also includes topics such as algorithmic mechanism design (Nisan and Ronen, I999) and the inefficiency of equilibria (Koutsoupias and Papadimitriou, I999). Algorithmic game theory finds application in a variety of domains such as networks (Roughgarden, 2005), security (Tambe, 20II), and information markets (Feigenbaum et al., 
2005). Though learning has been successfully employed as an iterative way to solve games (Akchurina, 2009), the foci of multiagent learning and algorithmic game theory are very distinct. Algorithmic game theory yields algorithms, centralized or distributed, that compute solutions to a game. Multiagent learning studies intelligent agents with limited information that learn to adapt through interaction, which may or may not result in equilibrium play.

\section{Optimal Adaptive Control}

An adaptive controller adapts to a system with time-varying or initially unknown parameters. In optimal adaptive control, the controller's objective is to minimize the cost function over the system's state space. If the control system is linear and the cost function quadratic, the optimal controller is found by solving the algebraic Riccati equation. For non-linear control problems, one must solve the Hamilton-Jacobi equation. As a reinforcement learner is essentially a controller that adapts its behavior subject to a reward function of the system, optimal adaptive control can be realized as reinforcement learning (Sutton et al., I991; Khan et al., 20I2). Vamvoudakis and Lewis (20II) extend this relation to multiagent learning and present an online adaptive control algorithm for general sum games. The algorithm is based on a separate actor/critic parametric network approximator for each player and converges to the coupled Riccati and Hamilton-Jacobi equations for linear and nonlinear systems respectively.

\section{I.5 Contributions and Dissertation Outline}

This dissertation is organized in two parts. In Part l, we investigate evolutionary game theory as a formal framework to model the dynamics of multiagent learning. In particular, we devise evolutionary models of individual reinforcement learners in dynamic games. In Part Il, we focus on the application of said models to real world domains.

Chapter 2 provides an introduction to the formal concepts and key results of multiagent learning. We define the fundamental models of single- and multiagent learning, i.e., Markov decision processes and stochastic games. Furthermore, we give an introduction to value and policy iteration as well as $Q$-learning and learning automata. The chapter concludes with a brief yet comprehensive survey and categorization of multiagent learning algorithms in Section 2.6.

In Chapter 3, we explore the relation between evolutionary game theory and multiagent reinforcement learning. We discuss two key concepts of evolutionary game theory, i.e., evolutionarily stable strategies and replicator dynamics. In Section 3.3, we show the formal relation between games of learning automata and asymmetric continuous time replicator dynamics as well as the link between $Q$-learning and selection- 
mutation dynamics. In Section 3.4, we advance existing work by proving that a large collective of learning agents can be approximated by the symmetric continuous time replicator dynamics. The formal relation is affirmed by a selection of experiments for both single- and multi-population dynamics.

In Chapter 4, we extend the link between multiagent reinforcement learning and evolutionary game theory to multi-state games. In particular, we introduce the average reward game and state-coupled replicator dynamics for irreducible stochastic games. To the best of our knowledge, our work presents the first evolutionary model for sequential multi-state decision making. The average reward game aggregates payoff information by averaging stage rewards over the interim immediate rewards. Statecoupled replicator dynamics use direct state-coupling by incorporating these expected average payoffs in all states under current strategies, weighted by the frequency of state occurrences. Results presented in this chapter have been published in various conference and workshop proceedings (Hennes et al., 2008, 2009b, 2010).

In Chapter 5, we analyze strategic interactions in settings that include multiple actors, many possible actions, and relationships among goals, tasks and resources. In particular, we introduce meta-strategies as a method to reduce large-scale strategic interactions to bilateral normal-form games with a significantly smaller strategy space, while preserving many of the strategic characteristics of the original setting. This work has been published in Hennes et al. (2009b) and de Jong et al. (20IIa,b).

In Chapter 6, we analyze the competitive advantage of price signal information for traders in simulated double auctions. More information does not guarantee higher performance, in particular, traders with limited information perform below market average and are outperformed by random traders; only insiders beat the market. We analyze the market dynamics with an evolutionary model of agents with competing information levels. Results show that the highest information level will dominate if information comes for free. If information is costly, less-informed traders may prevail reflecting a more realistic distribution over information levels. The work presented in Chapter 6 is based on Hennes et al. (2012a).

In Chapter 7, we perform an evolutionary analysis of collision avoidance based on the velocity obstacle paradigm. The velocity obstacle is a geometric representation of all velocities that will eventually result in a collision given that the dynamic obstacle maintains the observed velocity. We compare three variations of the velocity obstacle in a competitive setting and identify which of the techniques is most robust. The work presented in Chapter 7 builds on and is published in parts in Claes et al. (20II, 20I2b) and Hennes et al. (2012b, 2013).

Chapter 8 concludes this dissertation. We reflect on the research questions and the original contributions presented in this dissertation. Finally, we discuss limitations and open challenges and give directions for future work. 
Part

Formal Framework 



\section{2 Fundamentals of Multiagent Learning}

This chapter provides an introduction to the formal concepts and some key results of multiagent learning. The learning process of an adaptive agent within a multiagent system can be modeled as a reinforcement learning task. The agent iteratively interacts with the environment and other agents while observing feedback from the environment. In order to maximize expected long term reward, the action behavior is adapted according to this reinforcement signal. In contrast to supervised learning the agent is not provided with the correct or optimal state-action pair at any time. Most singleagent reinforcement learning techniques are modeled as sequential decision making based on the formal framework of Markov decision processes (Puterman, 2005). Valueiteration and policy-iteration are two algorithms that compute an optimal policy for a Markov decision process given full information. If full information is not available, the agent's objective is to learn an optimal policy. We call this type of technique singleagent model-free reinforcement learning, of which Q-learning (Watkins, I989) is a well-known example. Along with Q-learning, which follows the value-iteration scheme, we discuss the learning automaton (Narendra and Thathachar, I974) - a model-free reinforcement learner based on policy iteration. As Markov decision processes include only one decision maker, this framework is insufficient for modeling multiagent interactions. Stochastic games (Shapley, I953; Thuijsman, I992; Filar and Vrieze, I996) provide a generalization to multiple agents. Nash Q-learning ( $\mathrm{Hu}$ and Wellman, 2003), an extension of single-agent Q-learning, is one prominent example of multiagent learning algorithms based on stochastic games.

This chapter is organized as follows. We provide a formal definition of Markov decision processes in Section 2.I. In Section 2.2 we discuss optimization based on valueiteration and policy-iteration. Q-learning and learning automata are introduced in Section 2.3 and 2.4 respectively. Section 2.5 defines general-sum stochastic games. Section 2.6 gives a brief account of multiagent learning algorithms and summarizes key results. 


\section{I Markov Decision Processes}

Markov decision processes formulate sequential decision making under uncertainty of a single decision maker. The decision maker, also called agent or controller, observes the system state at each stage of the process. The agent chooses an action from its admissible action-set, which may or may not be the same in all states. An immediate reward is received (or cost is incurred) and the system transitions to the next stage. At this point the system may reside in the same or a different state. Both the reward and the transition probability depend on the last state and the choice of action.

For a full account of discrete time Markov decision processes, the interested reader is advised to consult Puterman (2005) or, for an algorithmic perspective, Kolobov (2012). White (I993) surveys a collection of papers on the application of Markov decision processes, examples range from water resource management, through epidemics, to sports. To allow for a self-contained but yet concise recap, only fully observable Markov processes are addressed below. Partially observable Markov decision processes are discussed in Kaelbling et al. (I998).

Definition I. A Markov decision process is an agent controlled discrete time stochastic process defined by the quadruple $\langle S, A, R, T\rangle$. At each stage $t \in \mathbb{N}^{0}$, the agent observes the current state $s_{t} \in S$ and chooses an action $a_{t} \in A$. The process transitions to the next state $s_{t+1}$ according to the transition function $T: S \times A \mapsto \triangle(S) .{ }^{\mathrm{I}}$ The agent receives an immediate reward according to the reward function $R: S \times A \mapsto \mathbb{R} .^{2}$

The transition function $T$ is action and state dependent and maps to a probability distribution over the set of states $S$. In other words, the probability that the system transitions to some state $i$ at time $t+1$ is the $i$-th entry of the vector $T\left(s_{t}, a_{t}\right)$, i.e.:

$$
\operatorname{Pr}\left(s_{t+1}=i \mid s_{t}, a_{t}\right)=T_{i}\left(s_{t}, a_{t}\right)
$$

The conditional probability of future states depends only on the present, not on the sequence of previous states. Therefore, we call the process memoryless or Markovian.

When history is of no relevance, a deterministic and stationary policy can be written as a function of the current state, i.e. $\pi: S \rightarrow A$. The current stage action is generated by $a_{t}=\pi\left(s_{t}\right)$ and the state transitions according to:

$$
s_{t+1} \sim T\left(s_{t}, \pi\left(s_{t}\right)\right)
$$

The agent's objective is to identify a policy maximizing long term reward from a given initial state. It is essential to also consider future rewards rather than just con-

\footnotetext{
${ }^{\mathrm{I}} \triangle(\cdot)$ defines the set of probability distributions over a set.

${ }^{2}$ The reward can alternatively be defined to depend on the next state, i.e. $R: S \times A \times S \mapsto \mathbb{R}$.
} 
sidering the immediate reward a certain action has triggered. Taking the regret of a low immediate reward might be beneficial in the long run. The return of a Markov decision process is usually a cumulative criterion over discounted or averaged stage rewards.

The total $\beta$-discounted expected reward, with discount factor $\beta \in[0,1)$, in an infinite horizon Markov decision process $\langle S, A, R, T\rangle$ under stationary deterministic policy $\pi$ is defined as:

$$
V(s, \pi)=\mathbb{E}\left[\sum_{t=0}^{\infty} \beta^{t} R\left(s_{t}, \pi\left(s_{t}\right)\right) \mid s_{0}=s\right],
$$

where $V$ is commonly referred to as the value function. The discount factor determines to which extend immediate rewards are preferred over future stage rewards. Discounting results in favorable contraction properties with regard to dynamic programming.

For the same process, the limiting average expected reward is given by:

$$
V(s, \pi)=\liminf _{T \rightarrow \infty} \frac{1}{T} \mathbb{E}\left[\sum_{t=0}^{T-1} R\left(s_{t}, \pi\left(s_{t}\right)\right) \mid s_{0}=s\right] .
$$

The average reward gives equal weight to all immediate rewards received over time and does not discount rewards at the horizon. If $\beta \rightarrow 1$, the discounted reward (2.2), when appropriately scaled, approximates the average reward (2.3). For an in-depth discussion of average and discounted reward criteria, see Tsitsiklis and Roy (2002).

We here consider the case where the decision maker is maximizing discounted rewards. The value function (2.2) is the unique solution of the Bellman Equation (Bellman, 1957) of the form:

$$
V(s, \pi)=\sum_{s^{\prime} \in S} T_{s^{\prime}}(s, \pi(s))\left[R(s, \pi(s))+\beta V\left(s^{\prime}, \pi\right)\right], \quad \forall s \in S .
$$

Finding an optimal policy is the crux of Markov decision processes. We define optimality on the basis of the optimal value function. The optimal value function $V^{*}$ of a Markov decision process is given by:

$$
V^{*}(s)=\max _{\pi} V(s, \pi), \quad \forall s \in S,
$$

and corresponds to the Bellman Equation of the form:

$$
V^{*}(s)=\max _{a \in A} \sum_{s^{\prime} \in S} T_{s^{\prime}}(s, a)\left[R(s, a)+\beta V^{*}\left(s^{\prime}\right)\right], \quad \forall s \in S .
$$

The optimal value $V^{*}(s)$ is the best expected total discounted reward that can be obtained from state $s$. 
Definition 2. A policy $\pi^{*}$ is optimal if and only if

$$
\pi^{*}(s) \in \underset{a \in A}{\arg \max } \sum_{s^{\prime} \in S} T_{s^{\prime}}(s, a)\left[R(s, a)+\beta V^{*}\left(s^{\prime}\right)\right], \quad \forall s \in S .
$$

That is to say that $\pi^{*}$ determines an optimal choice of action in every state and results in maximum expected return, i.e. $V\left(s, \pi^{*}\right)=V^{*}(s)$ for all $s \in S$.

\subsection{Value Iteration and Policy Iteration}

Value iteration and policy iteration are both algorithms that iteratively compute the value function of a given Markov decision process.

Value iteration (Bellman, I957) starts from an arbitrary value function $V_{0}$ and refines the state value estimations in each step according to the following equation:

$$
V_{t+1}(s)=\max _{a \in A}\left[R\left(s_{t}, a\right)+\gamma \sum_{s^{\prime} \in S} T_{s^{\prime}}\left(s_{t}, a\right) V_{t}\left(s^{\prime}\right)\right], \quad \forall s \in S, t \geq 0
$$

It can be shown that the series $\left(V_{t} ; t \geq 0\right)$ converges to the optimal value function $V^{*}$. As the convergence proof is beyond the scope of this thesis, we will only provide a supporting intuition. Based on the Bellman equation (2.6), we define a contraction mapping $\mathscr{T}$ with contraction factor $\gamma$ for a generic function $f: \mathbb{R}^{|S|} \rightarrow \mathbb{R}^{|S|}$. Next, we rewrite (2.7) using this contraction mapping, also called the Bellman operator:

$$
V_{t+1}=\mathscr{T} V_{t}
$$

From the Banach fixed-point theorem it follows that the series (2.8) converges to the unique fixed point $V^{*}$ at a geometric rate, i.e., for any arbitrary initial value function $V_{0} \in \mathbb{R}^{|S|}$ :

$$
\lim _{t \rightarrow \infty}(\mathscr{T})^{t} V_{0}=V^{*}
$$

For the full proof and accompanying definitions, the reader may consult Bellman (I957), Russell and Norvig (2010), and Szepesvári (2010). Once the optimal value function $V^{*}$ is known, an optimal policy $\pi^{*}$ directly follows from Definition 2. In practice, (2.7) is calculated until for two successive iterations $t$ and $t+1$ the following threshold criterion is reached:

$$
\max _{s \in S}\left|V_{t}(s)-V_{t+1}(s)\right|<\epsilon,
$$

where $\epsilon \in \mathbb{R}$ is some small number. A smaller threshold $\epsilon$ results in a better approximation of the optimal value function but comes at the cost of a longer runtime. Naturally, when $\epsilon \rightarrow 0$ then $V_{t+1} \rightarrow V^{*}$. 
Similar to Definition 2, we can define the following greedy policy with respect to $V_{t}$ at any given step $t$ of the value iteration process:

$$
\pi_{t}(s)=\underset{a \in A}{\arg \max }\left[R(s, a)+\gamma \sum_{s^{\prime} \in S} T_{s^{\prime}}(s, a) V_{t}\left(s^{\prime}\right)\right] .
$$

Howard (I960) observes that the greedy policy often becomes exactly optimal long before the value iteration has converged; a problem which is addressed by policy iteration. Initially, an arbitrary policy $\pi_{0}$ is fixed. The fixed policy is evaluated by solving the Bellman equation (2.4) in form of a system of linear equations. Subsequently, a corresponding updated greedy policy $\pi_{t+1}$ is devised and the process is repeated. The stopping criteria is reached when two subsequent policies are equal, i.e., $\pi_{t+1}(s)=\pi_{t}(s), \forall s \in S$.

For large state spaces, solving 2.4 as a system of linear equations may become intractable. Modified policy iteration (Puterman and Shin, 1978) uses a modified form of value iteration for the policy evaluation. Value estimates are updated iteratively, however, (2.7) is adapted to use the fixed policy $\pi_{t}$ rather than computing the current best action in each iteration. For a discussion on the number of iterations required in each policy evaluation step see Puterman (2005).

\subsection{Q-Learning}

So far, we have assumed that both the reward function $R$ and the state transition function $T$ are known to the decision maker. If the agent does not know either one, the agent's objective becomes to learn an optimal policy. Q-learning (Watkins, I989; Watkins and Dayan, I992) is one example of model-free reinforcement learning and thus without the requirement for a specific model of the environment.

Q-learning provides a simple way to learn optimal policies by successively improving the evaluation of state-action pairs. Watkins and Dayan (1992) proof that Q-learning converges to the optimal policy in Markov decision processes with finite state and action spaces as long as all actions are repeatedly sampled in all states. $Q$ values, stateaction pair evaluations, or simply action values are defined by:

$$
Q^{\pi}(s, a)=R(s, a)+\gamma \sum_{s^{\prime} \in S} T_{s^{\prime}}(s, a) V\left(s^{\prime}, \pi\right) .
$$

The $Q$ value is the expected discounted reward for choosing action $a$ in state $s$ and following policy $\pi$ thereafter. Let $Q^{*}$ be the action values that satisfy the system of linear equations (2.II) for an optimal policy $\pi^{*}$. It follows from (2.6), that

$$
\begin{aligned}
V^{*}(s) & =\max _{a \in A}\left[R(s, a)+\gamma \sum_{s^{\prime} \in S} T_{s^{\prime}}(s, a) V^{*}\left(s^{\prime}\right)\right] \\
& =\max _{a \in A} Q^{*}(s, a) .
\end{aligned}
$$


Furthermore, the optimal policy $\pi^{*}$ is determined by choosing the action with the highest $Q$ value in each state:

$$
\pi^{*}=\underset{a \in A}{\arg \max } Q^{*}(s, a), \quad \forall s \in S
$$

Hence, if the agent can learn the unique $Q^{*}$ values, an optimal policy is easily obtained.

\section{Update rule and action selection}

$Q$-learning uses the following update rule to iteratively improve value estimates and eventually learn the optimal $Q^{*}$ values:

$$
Q_{t+1}\left(s_{t}, a_{t}\right)=\left(1-\alpha_{t}\right) Q_{t}\left(s_{t}, a_{t}\right)+\alpha_{t}\left[R\left(s_{t}, a_{t}\right)+\gamma V_{t}\left(s_{t+1}\right)\right],
$$

where

$$
V_{t}\left(s_{t+1}\right)=\max _{a^{\prime} \in A} Q_{t}\left(s_{t+1}, a^{\prime}\right) .
$$

Watkins and Dayan (I992) proof that $Q(s, a) \rightarrow Q^{*}(s, a)$ as $t \rightarrow \infty, \forall s \in S, a \in A$, given that each state-action pair $(s, a)$ is visited infinitely often.

Note that the update rule (2.I4) is independent of the policy. $Q$ values are updated according to the optimal action based on current estimates $Q_{t}$ in (2.II). Therefore, $Q$ learning belongs to the class of off-policy learners. The $Q$ values are not affected by the policy used for action sampling. In contrast, SARSA (Sutton and Barto, I998) is an example for on-policy learning. As such, the value of the policy $\pi$ in use is learned. SARSA factors the action selection into the update rule, i.e., $Q$ values are updated using the quintuple $\left\langle s_{t}, a_{t}, R_{t}, s_{t+1}, a_{t+1}\right\rangle$, where $a_{t+1}$ is sampled from the same policy as the original action:

$$
Q_{t+1}\left(s_{t}, a_{t}\right)=\left(1-\alpha_{t}\right) Q_{t}\left(s_{t}, a_{t}\right)+\alpha_{t}\left[R_{t}+\gamma Q_{t}\left(s_{t+1}, a_{t+1}\right)\right] .
$$

The SARSA state value estimation at stage $t$ is:

$$
V_{t}\left(s_{t}\right)=\sum_{a \in A} \pi\left(s_{t}, a\right) Q\left(s_{t}, a\right)
$$

where $\pi(s, a)$ is the probability of taking action $a$ in state $s$. Action $a_{t}$ is drawn from the non-deterministic policy $\pi$, i.e. $a_{t} \sim \pi\left(s_{t}, \cdot\right)$. Any non-deterministic policy $\pi$ satisfies the following two constraints:

$$
\begin{aligned}
\forall a \in A, s \in S: & \pi(s, a)>0, \\
\forall s \in S: & \sum_{a \in A} \pi_{t+1}(s, a)=1 .
\end{aligned}
$$


Since $Q$-learning is an off-policy learner, various action sampling schemes can be used without loss of asymptotical convergence as long as each state is visited infinitely often. Two common strategies are $\epsilon$-greedy action selection and Boltzmann exploration. In $\epsilon$-greedy action selection, the action $a_{t}^{*}=\arg \max _{a} Q_{t}\left(s_{t}, a\right)$ in stage $t$ and state $s_{t}$ is selected with probability $\operatorname{Pr}\left(a_{t}=a^{*} \mid Q_{t}\right)=1-\epsilon$. All other actions are sampled with uniform probability $\frac{\epsilon}{\left|A_{s}\right|-1}{ }^{3} Q$ value estimates are expected to be inaccurate in early stages of the learning process. As a direct consequence, a pure greedy policy that only exploits current but incorrect knowledge never samples seemingly inferior actions. A policy with $\epsilon$-greedy action selection is always exploring and thus less likely to get stuck in local minima. However, exploring does come at a cost. By choosing random actions with $\epsilon$ probability, the agent risks the regret of a low immediate reward. Determining the right trade-off between exploration and exploitation remains far from straightforward and is thus known as the exploration-exploitation dilemma (Kaelbling et al., I996; Maes et al., 20I2).

The Boltzmann exploration scheme, also called softmax action selection, uses a Boltzmann distribution to generate action probabilities:

$$
\pi\left(s_{t}, a\right)=\operatorname{Pr}\left(a_{t}=a \mid Q_{t}\right)=\frac{e^{Q_{t}(s, a) / \tau}}{\sum_{a^{\prime} \in A} e^{Q_{t}\left(s, a^{\prime}\right) / \tau}},
$$

where $\tau \in \mathbb{R}^{+}$is called the temperature parameter, used to balance exploitation and exploration. Temperature may also be some function of $t$, in this case we speak of Boltzmann exploration with varying temperature. In the limit $\tau \rightarrow 0$, Boltzmann exploration becomes equivalent to pure greedy action selection.

It should be noted that neither method is clearly superior to the other. Both $\epsilon$ greedy action selection and Boltzmann exploration (with fixed temperature) require tuning of one parameter to the problem at hand. While $\epsilon$ in $\epsilon$-greedy is independent of the range of values that $Q$ may take on, the set of possible rewards must be considered when setting the temperature $\tau$ for the Boltzmann distribution.

Q-learning as well as SARSA use only one step of temporal-difference backup. In other words, the update rule uses only information of the immediately following state $s_{t+1} \cdot \operatorname{TD}(\lambda)$ (Sutton, I988) uses multiple subsequent states to update the current value estimates. As future states, beyond $s_{t+1}$, are not known to the agent, on-line $\operatorname{TD}(\lambda)$ uses eligibility-traces to approximate multi-step temporal-difference backup (Sutton and Barto, 1998). Each state is associated with an eligibility trace $e_{t}(s) \in \mathbb{R}^{+}$which marks the state as "eligible" for the ongoing learning process. At each stage $t$, all traces are decayed by the factor $\gamma \lambda$ and only trace $e_{t}\left(s_{t}\right)$ is increased by 1 . Hence, $\lambda \in[0,1]$ is

\footnotetext{
${ }^{3}$ In some works, the agent selects an action (including the optimal) at random with $\epsilon$ probability instead of only from the remaining set $A_{s} \backslash a^{*}$.
} 
referred to as the trace-decay parameter. $\operatorname{In} \operatorname{TD}(\lambda)$ learning, value changes are proportional to the eligibility traces and all states with a nonzero trace are updated at each stage:

$$
V_{t+1}(s)=V_{t}(s)+\alpha e_{t}(s)\left[R_{t}+\gamma V_{t}\left(s_{t+1}\right)-V_{t}\left(s_{t}\right)\right], \forall s \in S .
$$

Eligibility traces can also be used in combination with $Q$-learning and SARSA, e.g., $Q(\lambda)$ and $\operatorname{SARSA}(\lambda)$. For more information consult the works by Watkins (I989) and Sutton and Barto (I998) respectively.

\subsection{Learning Automata}

A learning automaton is a simple adaptive control mechanism operating in unknown stochastic environments. The study of learning automata dates back to the work by Bush and Mosteller (I955) in the context of mathematical psychology; engineering applications are first explored by Tsetlin (I962). The term learning automata appears to have been coined later by Narendra and Thathachar (I974). Learning automata are mostly applied as adaptive decision making units in the engineering domain (Najim and Poznyak, I994; Narendra and Thathachar, I989; Thathachar and Sastry, 2002) but also studied as a basis for multi-agent reinforcement learning (Nowé et al., 2006).

In the context of reinforcement learning, learning automata follow the policyiteration scheme. An initially random policy is used to explore the environment; by monitoring the reinforcement signal the policy is gradually improved in order to maximize the expected reward. The analysis in this thesis is restricted to finite action-set learning automata. For continuous action reinforcement learning automata see, for example, Santharam et al. (I994), or Howell and Gordon (200I). Furthermore, only environments with real-valued reward functions are considered. Narendra and Thathachar (I989) classify environments into three models based on the type of reinforcement signal. The P-model corresponds to binary reward signals, the $Q$-model describes environments with a finte-set of reward values, while real-valued rewards fall under the $S$-model.

\section{Finite Action Set Learning Automata}

The class of finite action-set learning automata considers only automata that optimize their policies over a finite action-set $A=\{1, \ldots, k\}$ with $k$ some finite integer. One optimization step, called epoch from here on, is divided into two steps: action selection and policy update. At the beginning of an epoch $t$ the automaton draws a random action $a_{t}$ according to the probability distribution $\pi_{t}$. Based on the action $a_{t}$ the environment responds with a reinforcement signal $R_{t}$. Hereafter the automaton uses the reward $R_{t}$ to update $\pi_{t}$ to the new policy $\pi_{t+1}$. The update rule for finite action set 
learning automata is given below:

$$
\pi_{t+1}(a)=\pi_{t}(a)+\left\{\begin{array}{cl}
\alpha R_{t}\left(1-\pi_{t}(a)\right)-\beta\left(1-R_{t}\right) \pi_{t}(a) & \text { if } a=a_{t} \\
-\alpha R_{t} \pi_{t}(a)+\beta\left(1-R_{t}\right)\left[(k-1)^{-1}-\pi_{t}(a)\right] & \text { if } a \neq a_{t},
\end{array}\right.
$$

where $\alpha \in[0,1]$ and $\beta \in[0,1]$ are the reward and penalty parameters respectively. If $\alpha=\beta$, the update scheme is referred to as linear reward-penalty $\left(L_{R-P}\right)$; for $\beta=0$, it is called linear reward-inaction $\left(L_{R-I}\right)$; if $\beta$ is chosen to be small compared to $\alpha$, it is called linear reward- $\epsilon$-penalty $\left(L_{R-\epsilon P}\right)$. With $R_{t} \in[0,1]$, the update rule (2.2I) does indeed result in a valid probability distribution $\pi_{t+1}$ satisfying the constraints (2.I8).

\section{Networks of Learning Automata}

A finite action set learning automaton operates by receiving the reinforcement signal and is not aware of any state information. In Markov decision processes the optimal choice of action clearly depends on the current environment state. The work by Wheeler and Narendra (I986) extends finite action set learning automata in order to enable sequential decision making in Markov processes.

In networks of automata control is passed on from one automaton to another. An agent associates a dedicated learning automata to each state of the environment. This automaton optimizes the policy in that state using the standard update rule given in (2.2I). At each stage $t$ only a single automaton is active and selects the action $a_{t}$. However, the immediate reward from the environment is not directly fed back to the learning automaton. Instead, when the automaton becomes active again, i.e., next time the same state occurs, it is informed about the cumulative reward gathered since the last activation and the time that has passed. The delayed policy update is performed before a new action is sampled. The modified reward feedback $\bar{R}_{t}(s)$ for automaton $\mathscr{A}(s)$ associated with state $s$ is given by:

$$
\bar{R}_{t}(s)=\frac{\Delta r}{\Delta t}=\frac{\sum_{i=l(s)}^{t-1} R_{i}}{t-l(s)}
$$

where $R_{t}$ is the immediate reward in stage $t$ and $l(s)$ is the last occurrence function that determines in which stage state $s$ was visited last. The reward feedback in stage $t$ equals the cumulative reward $\Delta r$ divided by time-frame $\Delta t$. The cumulative reward $\Delta r$ is the sum over all immediate rewards gathered in all states beginning with epoch $l(s)$ and including the last epoch $t-1$. The time-frame $\Delta t$ measures the number of epochs that have passed since automaton $\mathscr{A}(s)$ has been active last. This means the state policy is updated using the previous action in this state $a_{l(s)}$ and the average stage reward over the interim immediate rewards. 


\subsection{Stochastic Games}

The work by Shapley (I953) is the first to introduce games played by a finite number of players, on a finite state space with probabilistic state changes. In each stage of the game, each player plays one of finitely many actions. The simultaneously played actions form the joint action, or action profile, which determines the reward for each player. A transition function maps action profiles to a distribution over states, which drives the probabilistic state changes. The payoff of the game is a function of the sequence of rewards, i.e., the discounted reward, limit average reward or total reward. In other words, stochastic games generalize the framework of Markov decision processes to multiple agents.

The relationship between stochastic games and Markov decision processes as well as Markov chains is treated in great detail in the work by Neyman (2003). We highlight two important observations:

I. The dynamics of the states of a stochastic game form a Markov chain whenever the players' policies are stationary.

2. The decision problem faced by a player in a stochastic game when all other players choose a fixed profile of stationary strategies is equivalent to a Markov decision process.

Definition 3. The tuple $\left\langle n, S, A^{1}, \ldots, A^{n}, R^{1}, \ldots, R^{n}, T\right\rangle$ defines a stochastic game $G$ with $n$ players. At each stage $t$, player $i$ perceives the current state $s_{t} \in S$ and chooses an action $a_{t}^{i}$ from the admissible action set $A^{i}$. The payoff function $R^{i}: S \times A^{1} \times \cdots \times A^{n} \mapsto \mathbb{R}$ maps the joint action $a_{t}=\left\langle a_{t}^{1} \ldots a_{t}^{n}\right\rangle$ to an immediate payoff value for player $i$. The transition function $T: S \times A^{1} \times \cdots \times A^{n} \mapsto \triangle(S)$ determines the probabilistic state change to the next state $s_{t+1}$.

In this work we restrict our consideration to irreducible stochastic games, i.e., the set of games where there is only one ergodic class independent of the strategies. The motivation for this restriction is two-fold. In the presence of more than one ergodic class one could analyze the corresponding sub-games separately. Furthermore, the restriction ensures that the game has no absorbing states. Games with absorbing states are of no particular interest with respect to evolution or learning since any type of exploration will always lead to absorption. A formal definition of an ergodic class is given below. 
Definition 4. In the context of a stochastic game $G=\left\langle n, S, A^{1}, \ldots, A^{n}, R^{1}, \ldots, R^{n}, T\right\rangle$, $E \subseteq S$ is an ergodic class if and only if the following conditions hold.

(a) $\forall s \in E: \operatorname{Pr}\left(s_{t+1} \in E \mid s_{t}=s\right)=1$

(b) $\forall E^{\prime} \subset E$, (a) does not hold.

We recall that a stochastic game, where all players employ stationary policies, reduces to a Markov chain. It directly follows that an irreducible stochastic game has a stationary distribution under stationary policies.

Definition 5. An irreducible stochastic game has a stationary distribution over states $x \in$ $\Delta(S)$ under stationary policies $\pi$ with:

$$
x_{s^{\prime}}=\sum_{s \in S} x_{s} p_{s, s^{\prime}}, \quad \forall s^{\prime} \in S,
$$

where $p_{s, s^{\prime}}$ is the transition probability from state $s$ to $s^{\prime}$ :

$$
p_{s, s^{\prime}}=\sum_{a \in A^{i} \times \ldots \times A^{n}}\left[T_{s^{\prime}}(s, a) \prod_{i=1}^{n} \pi^{i}\left(s, a^{i}\right)\right] .
$$

Note, that $x^{\top} P=x^{\top}$, where $P=\left[p_{i, j}\right]_{i \in S ; j \in S}$ is the transition matrix.

Besides irreducible stochastic games, another special case treated in this thesis are stateless games, i.e. $S=\emptyset$. In this specific class of games, state transition mappings are deprecated. Alternatively, one could also say that there is exactly one state and the game resides in this state during all stages, i.e. $S=\left\{s^{0}\right\}$ and $\operatorname{Pr}\left(s_{t}=s^{0}\right)=1, \forall t$. Stateless stochastic games are also called repeated games.

\subsection{Multiagent Learning Algorithms}

Single-agent reinforcement learning algorithms can be used in multiagent settings, like repeated and stochastic games, in a straightforward manner. Single-agent learners in multiagent games are also called individual and independent learners, i.e. they are not aware of the existence of other agents. As convergence guarantees do no longer hold, one has to be cautious with regard to their performance. However, single-agent reinforcement learning techniques have been applied successfully in multiagent domains, e.g., Matarić (1997); Yang and Gu (2005). In addition to this naïve approach, many multiagent reinforcement learning algorithms have been devised over the years. A brief survey is provided below. Being fairly comprehensive, the description is necessarily succinct; the reader is referred to selected sources for further details and examples (Panait and Luke, 2005; Shoham et al., 2007; Buşoniu et al., 2008; Tuyls and Weiss, 20I2). 


\section{Value Iteration in Stochastic Games}

The concept of value iteration can be extended to two player zero-sum stochastic games. In a zero-sum stochastic game the payoffs of all players sum up to zero (or some other constant) for each joint action in each stage game. If there are only two players the gain of one player becomes the other's loss. Let us assume the payoff function $R$ determines the amount player two has to pay player one. It follows that $V^{*}(s)$ denotes the value of the game, i.e. the amount player one receives in the long run given the game started in state $s$. Optimal $Q^{*}$ values for such a game are defined as:

$$
Q^{*}(s, a)=R(s, a)+\gamma \sum_{s^{\prime} \in S} T_{s^{\prime}}(s, a) V^{*}\left(s^{\prime}\right)
$$

where $Q^{*}$ is a function of the state $s$ and joint action $a=\left\langle a^{1}, a^{2}\right\rangle$. In Markov decision processes we can determine $V^{*}$ from $Q^{*}$ values according to (2.I2). However, games with multiple players require a more involved method than the max operator maximizing over action values. Shapley's algorithm (Shapley, I953; Bowling and Veloso, 2000) computes the stage game value in each iteration by solving a linear program. Stage game payoffs are represented by the $Q$ value for each joint action. The value for the stage game in state $s$ is:

$$
V^{*}(s)=\max _{\pi \in \triangle\left(A^{1}\right)} \min _{a^{2} \in A^{2}} \sum_{a^{1} \in A^{1}} \pi_{a^{1}} Q^{*}\left(s,\left\langle a^{1}, a^{2}\right\rangle\right) .
$$

Player one maximizes its strategy $\pi$ under the assumption that player two plays a best response. The algorithm is proven to return equilibrium strategy profiles for two player zero-sum stochastic games with discounting (Shapley, I953). Fictitious play (Brown, I95I; Vrieze, I987) and its extension smooth fictitious play (Fudenberg and Levine, I999; Gerding et al., 2008) fall in the same value iteration category. Pollatschek and Avi-Itzhak (1969) give a similar generalization to zero-sum stochastic games for the policy iteration scheme. It should be noted that all of the above algorithms require that the transition and payoff model are known and as such compute equilibrium policies rather than learn to play optimal.

\section{Minimax-Q Learning}

Minimax-Q, introduced by Littman (1994), is a reinforcement learning algorithm for two player zero-sum stochastic games. As such, the transition and reward functions are not required to be known by the agent. Leveraging the fact that payoffs balance out in zero-sum games, the agent only needs to observe its own payoff and can ignore the one of the opponent. However, in order to build a $Q$ value table as defined by (2.23), the full joint action profile is required information as well. 
Definition 6 (Claus and Boutilier (1998)). A joint action learner (JAL) is an agent that learns $Q$-values for joint actions as opposed to individual actions.

Without loss of generality, we can assume that player one is the agent under consideration and $R_{t}$ is the payoff to player one in stage $t$. The agent updates its state-jointaction estimates according to:

$$
Q_{t+1}\left(s_{t},\left\langle a_{t}^{1}, a_{t}^{2}\right\rangle\right)=\left(1-\alpha_{t}\right) Q_{t}\left(s_{t},\left\langle a_{t}^{1}, a_{t}^{2}\right\rangle\right)+\alpha_{t}\left[R_{t}+\gamma V_{t}\left(s_{t+1}\right)\right] .
$$

This learning rule is a straightforward extension of the original $Q$ learning update (2.I4) to two player zero-sum games. The value of the next state $V\left(s_{t+1}\right)$ follows from (2.24):

$$
V_{t+1}(s)=\max _{\pi \in \Delta\left(A^{1}\right)} \min _{a^{2} \in A^{2}} \sum_{a^{1} \in A^{1}} \pi_{a^{1}} Q_{t}\left(s,\left\langle a^{1}, a^{2}\right\rangle\right) .
$$

Littman (I994) proves that minimax- $Q$ converges to the game's Nash equilibrium under the same assumptions listed in Section 2.3. Minimax-SARSA, devised by Banerjee et al. (200I), is the on-policy equivalent of minimax-Q.

\section{Nash-Q Learning}

Nash-Q (Hu and Wellman, 1998,2003$)$ is an extension of $Q$-learning to non-cooperative multiagent settings based on the framework of general-sum stochastic games. Hu and Wellman (1998) define optimal Nash-Q values as the sum of immediate reward and discounted future rewards under the condition that all agents play a specified Nash equilibrium from the next stage onward. Just as minimax- $Q$, the algorithm estimates state-joint-action values, i.e. $Q$ is a function of states and joint-actions rather than simple state-action pairs. As there is no restriction on the payoff structure in generalsum games, each individual agent has to estimate $Q$ values for all other agents as well. The Nash-Q update rule is similar to (2.25), however, it is general in the number of agents and uses a different next-state value estimator $N a s h V_{t}^{i}$ :

$$
\begin{aligned}
Q_{t+1}^{i}\left(s_{t},\left\langle a^{1}, \ldots, a^{n}\right\rangle\right) & =\left(1-\alpha_{t}\right) Q\left(s_{t},\left\langle a^{1}, \ldots, a^{n}\right\rangle\right) \\
& +\alpha_{t}\left[R_{t}+\gamma \operatorname{Nash} V_{t}^{i}\left(s_{t+1}\right)\right] .
\end{aligned}
$$

A Nash equilibrium is computed for each stage game $\left(Q_{t}^{1}\left(s_{t+1}, \cdot\right), \ldots, Q_{t}^{n}\left(s_{t+1}, \cdot\right)\right)$ and results in the equilibrium payoff $\operatorname{Nash}_{t}^{i}\left(s_{t+1}, \cdot\right)$ to agent $i$. Agent $i$ updates its own $Q^{i}$ values according to (2.26) and furthermore uses the update rule to estimate $Q$ values for all other agents, i.e., $Q^{j} \forall j \in\{1, \ldots, n\} \backslash i$. Joint-action values $\left(Q^{1}(s, \cdot), \ldots, Q^{n}(s, \cdot)\right)$ form the payoff matrix of the normal form game played in state $s$. 
$\mathrm{Hu}$ and Wellman (I998) show that for two-player general-sum games the series $\left(Q_{t}^{1}, Q_{t}^{2}\right)$ updated by (2.26) converges to the Nash equilibrium $Q$ values under a set of assumptions. Each stage game that is encountered during the learning process either has a global optimal Nash equilibrium, or a saddle point. If one player deviates from a saddle point equilibrium, this player is worse off while all other players benefit (Hu and Wellman, I998). Bowling (2000) proofs that the convergence conditions are actually stronger and argues that all Nash equilibria must be optimal, or alternatively, all equilibria must be saddle points. In addition, the standard $Q$-learning assumptions must hold, i.e. each state-joint-action value is updated infinitely often. It should be noted that under these strong assumptions, convergence does not depend on the agents' action choices (Hu and Wellman, 2003), and therefore, action selection methods outlined in Section 2.3 may be used.

\section{Other Equilibrium Learning Algorithms}

Littman's Friend-or-Foe Q-learning has stronger convergence guarantees than Nash-Q, however, the method requires that other players are identified as being either "friend" or "foe" a priori (Littman, 20or). For two-player games, fully cooperative or fully competitive (i.e., zero-sum) learning is used as appropriate. For games with more than two players, a team of "friends" and a team of "foes" are formed. As "friends" are assumed to work together to maximize, and "foes" work together to minimize the agent's reward, the game can be reduced to a two-player zero-sum game with extended action sets.

Correlated-Q learning (CE-Q) (Greenwald and Hall, 2003) generalizes both Nash$Q$ and Friend-and-Foe $Q$-learning using the correlated equilibrium concept (Aumann, I959). In a correlated equilibrium, a coordinator chooses strategies for all players. The chosen strategies are stable, meaning no player has an incentive to deviate from the recommended strategy. Correlated equilibria of stage games can be computed efficiently by means of linear programming. However, only empirical convergence of Correlated$Q$ learning to equilibrium policies is shown.

As general-sum games do not feature balanced payoffs, each agent has to observe the immediate payoffs obtained by other players as well as its own for all of the above approaches. A further limitation of all equilibrium learning algorithms is that all agents have to adhere to the same equilibrium concept and if multiple equilibria exist in one stage game, coordination is required. Nash bargaining solutions solve the equilibrium selection problem. Qiao et al. (2006) propose NBS-Q learning and show empirical convergence to Nash bargaining solutions.

Optimal adaptive learning (OAL) (Wang and Sandholm, 2002) converges to an optimal Nash equilibrium in identical-interest stochastic games. In these fully cooperative games all agents share the same payoff function and the team objective is to find a joint policy so as to maximize the expected sum of discounted payoffs. 
Asymmetric-Q learning (Könönen, 2003) adopts the Stackelberg equilibrium concept. In Stackelberg games the strategy of the first player (leader) can be observed by the second player (follower), thus always playing a best response. Furthermore, the leader is aware that its strategy is observed and responded to rationally. This asymmetric information structure allows for stronger convergence proofs and requires less computation. However, this specific game model is not applicable in the general case.

\section{Gradient Ascent Optimization}

Singh et al. (2000) study a gradient ascent optimization algorithm in two-player, twoaction repeated games. More precisely, the dynamics of gradient ascent with an infinitesimal small step size are examined, thus giving rise to the name infinitesimal gradient ascent (IGA). The gradient ascent step in stage $t$ is defined as follows:

$$
\Delta \pi_{t}^{i}=\eta \frac{\partial V^{i}\left(\pi_{t}\right)}{\partial \pi_{t}^{i}},
$$

where $V^{i}$ are the expected payoffs for player $i$ under the strategy profile $\pi_{t}=\left\langle\pi_{t}^{1}, \pi_{t}^{2}\right\rangle$. Parameter $\eta$ is the policy-learning rate; in infinitesimal gradient ascent $\eta$ approaches 0 in the limit. The next stage policy is given by:

$$
\pi_{t+1}^{i}=W\left(\pi_{t}^{i}+\Delta \pi_{t}^{i}\right)
$$

where $W$ is a projection back onto agent $i$ 's valid policy space, i.e. $W: \mathbb{R}^{\left|A^{i}\right|} \rightarrow \triangle\left(A^{i}\right)$. The projection ensures that the updated policy remains valid even if the gradient step would drive it outside the simplex.

Singh et al. (2000) prove that infinitesimal gradient ascent either converges to a Nash equilibrium or average payoffs converge to the payoffs of the equilibrium. Bowling and Veloso (2002) provide a stronger convergence guarantee, i.e. universal convergence to Nash strategies, using a variable learning rate. Their algorithm WoLF-IGA uses the "Win or Learn Fast" principle: while winning, cautious strategy changes are taken - while loosing, a larger step size is used to quickly escape from the unfavorable situation. Losing and winning are defined with respect to the payoff of the Nash equilibrium solution which must be known a priori.

Further extensions of this work are generalized infinitesimal gradient ascent (GIGA) (Zinkevich, 2003) and GIGA-WoLF (Bowling, 2005). The former is an generalization to repeated games with more than two players, each with a finite action set. GIGA uses the same gradient step as defined in (2.27), however, the projection $W$ is generalized. GIGA-WoLF guarantees at most zero average regret, however policy convergence is only proven in a limited setting. The weighted policy learner (WPL) (Abdallah and Lesser, 2008) also uses an adaptive learning rate similar to (G)IGA-WoLF, how- 
ever, agents are not required to know the Nash equilibrium payoffs. The probability of choosing a good action increases by a rate that decreases as the probability approaches one (or the boundary of the simplex). Similarly, the probability of choosing a bad action decreases at a rate that also decreases as the probability approaches zero (Abdallah and Lesser, 2008).

All variants of gradient ascent require the computation of the partial derivative of the expected payoff with respect to the played strategy. The agent's expected payoff $V^{i}$ is a function of $\pi_{t}=\left\langle\pi_{t}^{1}, \pi_{t}^{2}\right\rangle$, which in turn results in the requirement for full observability of the opponent's action selection probability distribution, i.e. mixed strategy. However, the current mixed strategy of other agents are hardly observable in a standard multiagent learning task. Abdallah and Lesser (2008) argue that gradient ascent may be applied in a reinforcement learning task if the value function is approximated by a series $V_{t}^{i}$ as follows:

$$
V_{t+1}^{i}(\cdot)=\alpha R_{t}^{i}+(1-\alpha) V_{t}^{i}(\cdot)
$$

where $V_{t}^{i}(\cdot)$ is either a function of agent $i$ 's action $a_{t}^{i}$ or a function of $\pi_{t}$. The former is an oversimplification and invalidates any guarantees for the convergence of (2.28), the latter requires the aforementioned impracticable requirement for full strategy observability. Therefore, infinitesimal gradient ascent as defined in (2.27) and (2.28) may be used to solve a game iteratively but should not be regarded as a learning algorithm itself.

\section{Policy Hill-Climbing}

Policy hill-climbing (Bowling and Veloso, 2002) resembles gradient ascent but does not require direct observability of other players' strategies. The algorithm is an extension of an independent, individual $Q$-learner. In addition to the $Q$ values updated by (2.I4) a state-dependent policy $\pi_{t}^{i}(s, a)$ is maintained. The policy for agent $i$ is initialized as follows:

$$
\pi_{0}^{i}(s, a)=\frac{1}{\left|A^{i}\right|}, \quad \forall a \in A^{i}, s \in S .
$$

In each stage the policy is updated according to:

$$
\pi_{t+1}^{i}\left(s_{t}, a\right)=\pi_{t}^{i}\left(s_{t}, a\right)+\Delta \pi_{t}^{i}\left(s_{t}, a\right), \quad \forall a \in A^{i},
$$

where

$$
\Delta \pi_{t}^{i}\left(s_{t}, a\right)= \begin{cases}-\eta\left(s_{t}, a\right) & \text { if } a \neq \arg \max _{a^{\prime}} Q_{t}\left(s, a^{\prime}\right) \\ \sum_{a^{\prime} \neq a} \eta\left(s_{t}, a^{\prime}\right) & \text { otherwise }\end{cases}
$$




$$
\eta(s, a)=\min \left(\pi_{t}^{i}(s, a), \frac{\eta_{0}}{\left|A^{i}\right|-1}\right) .
$$

The policy $\pi$ is moved a step of at most $\eta_{0} \in(0,1]$ towards the greedy policy while constrained to a valid probability distribution. All actions that are not optimal according to $Q_{t}$ are reduced by (at most) $\eta_{0} /\left(\left|A^{i}\right|-1\right)$, while the optimal action is increased by (at most) $\eta_{0}$. Bowling and Veloso (2002) also introduce WoLF-PHC, the "Win or Learn Fast" variant of policy hill-climbing that uses a variable learning rate $\eta$. As the equilibrium payoff is not known by the agent, the expected value given the current policy is compared to the expected value of the average policy as an approximation for the equilibrium policy. Banerjee and Peng (2003) derive a different WoLF criterion which is accurately computable in two-player two-action games without knowing the equilibrium policies. Their policy dynamics based WoLF (PDWoLF) criterion also shows improvements for larger action spaces, however, in this case it is no longer guaranteed to be accurate.

\section{Other Adaptive Learners}

Conitzer and Sandholm $(2003,2007)$ propose AWESOME, a learning algorithm for repeated games that combines ideas from equilibrium learning (e.g. Nash- $Q$ learning) and adaptive learning (e.g. policy hill climbing). AWESOME follows the prinziple: Adapt when everybody is stationary, otherwise move to equilibrium. The algorithm assumes the structure of the game to be known (i.e. payoff functions for all players) and that the full joint-action is observable in every stage. AWESOME learns a best-response against (eventually) stationary opponents and converges to a Nash equilibrium in selfplay.

Hyper-Q learning (Tesauro, 2003) estimates values of mixed strategy profiles rather than joint-actions. Other agents' strategies are estimated via Bayesian inference. In the original work, a uniform grid discretization for mixed strategies is used, however, the underlying idea also translates to function approximators.

$M$-Qubed (Crandall and Goodrich, 20II) is a joint-action learner for repeated generalsum games. While repeated games are stateless, M-Qubed uses the last $\omega$ joint-actions to define an auxiliary state $s_{t}=\left\langle a_{t-\omega}, \ldots, a_{t-1}\right\rangle$, also called recurrent state (Moody et al., 2004). Recurrent states can help to improve cooperation in the form of indirect signaling through actions (Skyrms, 2003). M-Qubed is an on-policy learner, i.e. it uses the SARSA update rule (2.I6). The policy is chosen to balance best-response, cautious, and optimistic learning biases. M-Qubed has shown robust performance in a series of round-robin and evolutionary tournaments of two-player repeated games with up to 
three actions. However, as the recurrent state depends on the $\omega$ past joint-actions, the state space grows exponentially with $\omega$ and a growth factor of $\left|A^{1} \times, \ldots, \times A^{n}\right|$. It is therefore unclear how well M-Qubed copes in more complex games.

Learning algorithms such as (G)IGA-WoLF and M-Qubed, are designed for repeated games and not applicable in the more general class of stochastic games. Pepper (Crandall, 20I2) is a joint-action learner framework to extend such matrix learning algorithms to stochastic games. Similar to networks of learning automata, a matrix learning algorithm is used in each state $s \in S$ of the game. The learner in state $s$ attempts to maximize the reward given a suitable estimate of the joint-action values in this state. In particular, Pepper uses a combination of on-policy and off-policy state value estimation based on R-max (Brafman and Tennenholtz, 2002). Pepper has been successfully applied to M-Qubed, Salt (Karandikar et al., I998; Stimpson and Goodrich, 2003), fictitious play, and GIGA-WoLF.

\section{Limitations}

Convergence guarantees are mostly restricted to stateless repeated games (Singh et al., 2000; Zinkevich, 2003; Bowling, 2005) or are inapplicable in general-sum games (Littman, I994). Furthermore, many convergence proofs have strong assumptions with respect to the required a-priori knowledge or observability.

Equilibrium learners, e.g. minimax- $Q$ or Nash- $Q$, as well as policy hill-climbers focus on stage-wise solutions. Each state is solved separately and is only indirectly coupled to neighboring states using some form of next-state value estimates. It is unclear if and when such stage-wise solutions are applicable (Panait and Luke, 2005; Shoham et al., 2007; Buşoniu et al., 2008).

Buşoniu et al. (2008) argue that one important research goal is to identify and understand the conditions under which single-agent learners work in general-sum stochastic games. Especially, since these algorithms are predominantly used in reallife applications (Matarić, 1997; Yang and Gu, 2005).

Further limitations of the aforementioned algorithms are finite discrete state and action spaces as well as full state observability. Continuous spaces can be mapped to a tabular representation using tile-coding (Sutton and Barto, 1998). However, function approximation is an alternative that generally scales better than state- or actionspace discretization (Buşoniu et al., 20I0). The model of stochastic games is insufficient whenever the environment state is uncertain, i.e., state observations are noisy. State uncertainty is modeled explicitly in partially observable Markov decision processes and their multiagent complement, Dec-POMDPs (Oliehoek, 20Io). 


\subsection{Summary}

This chapter has introduced the concept of Markov decision processes, a simple model for sequential decision making. We have briefly explored the background of value and policy iteration. Two influential single-agent reinforcement learning algorithms have been introduced: both Q-learning and learning automata find frequent application as individual, independent learners in multiagent settings. Stochastic games deliver the fundamental framework for many multiagent learning algorithms. Finally, this chapter has provided a brief but fairly comprehensive survey of multiagent learning algorithms. 



\section{3 Evolutionary Models of Learning}

The learning performance of contemporary reinforcement learning techniques has been studied in great depth experimentally as well as formally for a diversity of single agent control tasks (Sutton and Barto, I998). Markov decision processes provide a mathematical framework to study single agent learning, however, they are not in general applicable to multi-agent learning. Once multiple adaptive agents simultaneously interact with each other and the environment, the process becomes highly dynamic and non-deterministic, thus violating the Markov property. Evidently, there is a strong need for an adequate theoretical framework modeling multi-agent learning. An evolutionary game theoretic approach has been employed to fill this gap (Tuyls and Parsons, 2007). In particular, Börgers and Sarin (1997) derive a formal relation between multiagent reinforcement learning and the replicator dynamics. This connection between replicators and reinforcement learning has been extended to different reinforcement learning algorithms such as learning automata and Q-learning (Tuyls et al., 2003b).

Exploiting the link between reinforcement learning and evolutionary game theory is beneficial for a number of reasons. The majority of state of the art reinforcement learning algorithms are blackbox models. This makes it difficult to gain detailed insight into the learning process and parameter tuning becomes a cumbersome task. Analyzing the learning dynamics helps to determine parameter configurations prior to actual deployment in the task domain. Furthermore, the possibility to formally analyze multi-agent learning helps to derive and compare new algorithms, which has been successfully demonstrated for lenient learners by Panait et al. (2008).

This chapter is organized as follows. We introduce evolutionary game theory and replicator dynamics in Section 3.I and Section 3.2. In Section 3.3 we discuss the asymmetric learning dynamics of reinforcement learning algorithms, in particular: learning automata, $Q$-learning, lenient $Q$-learning and regret minimization. Finally, we explore the relation between symmetric replicator dynamics and a large population of learning agents in Section 3.4. 


\section{I Evolutionary Game Theory}

Traditional game theory assumes perfectly rational and self-interested players. Consequently, classical game theory aims at finding an optimal strategy that maximizes the expected utility for a player. Adversaries are assumed to be rational as well and their optimal strategies are taken into consideration. Under this assumption, the equilibrium is mutual knowledge and the logical result of the game. ${ }^{\mathrm{I}}$ In contrast, evolutionary game theory is a descriptive approach. A game is played repeatedly by boundedly rational players with little or no knowledge of the game. Two randomly matched individuals play preassigned pure strategies according to their phenotype. In evolutionary game theory, the payoff determines the fitness (value of success) of the represented strategy, or phenotype, and is not interpreted as a preference. The evolutionary process is modeled using biological-inspired operators, i.e., natural selection, replication and mutation.

In nature, selection and replication is the process of survival of the fittest and mutation is caused by genetic defects. These mechanisms translate to economics in a straightforward manner: economical survival, experimentation, innovation and mistakes (Weibull, I997). Evolutionary processes also play an important role in social or cultural learning. In the social context, evolution is often understood as cultural evolution, meaning the dynamic change of concepts, ideas or behavior over time. Boyd and Richerson (1985) define culture as the information affecting the phenotype acquired by individuals through imitation or teaching. Evolution can also mirror the effects of individual learning. An adaptive agent has to choose from a set of possible strategies. Which strategies are favored over others depends on the experience the agent has previously gathered by interacting with the environment and other agents. The pool of possible strategies can be regarded as a population in the evolutionary perspective. The dynamical change of preferences within this set of strategies is driven by the evolution of the population. In general, any process for which the dynamics are described by selection, replication, and mutation, is termed evolutionary and the three mechanisms can be appropriately interpreted for a variety of domains.

We proceed by introducing the two core concepts of evolutionary game theory, evolutionarily stable strategies (Maynard Smith, I974, I982) and replicator dynamics (Taylor and Jonker, 1978). For a detailed discussion of evolutionary game theory, we refer the interested reader to Weibull (I995), Hofbauer and Sigmund (I998), and Sandholm (2OII).

\footnotetext{
${ }^{\mathrm{I}}$ If the game has multiple equilibria, classical game theory still suffers from the equilibrium selection problem.
} 


\section{Evolutionarily Stable Strategies}

In his seminal paper, Maynard Smith (I974) defines the concept of evolutionarily stable strategies in population games. A population game describes the dynamics of a population of individuals playing a game in competition with each other. Each individual uses one of finitely many pure strategies, numbered 1 to $k$. The state of the population is captured by $x=\left(x_{1} \cdots x_{k}\right)^{\top}$, where $x^{i}$ is the fraction of the population playing strategy $i$. The valid state space of the population is given by the $(k-1)$-simplex: $x \in \Delta^{k-1}=\left\{\left(x_{1} \cdots x_{k}\right)^{\top} \in \mathbb{R}^{k} \mid \sum_{i=1}^{k} x_{i}=1 \wedge \forall i: x_{i} \geq 0\right\}$. The payoff to an individual playing strategy $i$ in state $x$ is given by the fitness function $f\left(e_{i}, x\right)$, where $e_{i}$ is the $i$-th unit vector. The fitness of a mixed strategy $y \in \Delta^{k-1}$ in the population $x$ is given by $f(y, x)$.

We call a strategy $x$ evolutionarily stable if it cannot be invaded by any mutant $y \neq x$ provided that the population share of the mutant is sufficiently small. Initially the entire population plays some (mixed) strategy $x$. At some point in time a mutant strategy $y$ occurs and is played by a small fraction $\epsilon$ of the population. The mutant might invade the population (unstable), or it might become extinct due to natural selection (stable). A formal definition of evolutionary stability is given below.

Definition 7. A strategy $x$ is an evolutionarily stable strategy $(E S S)$ iff $\forall y \neq x: \exists \bar{\epsilon}_{y} \in(0,1)$ such that

$$
\forall \epsilon \in\left(0, \bar{\epsilon}_{y}\right): f(x, \epsilon y+(1-\epsilon) x)>f(y, \epsilon y+(1-\epsilon) x) .
$$

A strategy is protected against invasion if and only if the post-entry payoff of the mutant strategy $y$ in the population mixture $\epsilon y+(1-\epsilon) x$ is lower than the payoff of the original strategy $x$.

We can show that the strategy "defect" is evolutionarily stable in the prisoners' dilemma (see Example I). Table I.I shows the payoffs in a single lattice or bimatrix, i.e, the payoff for the row and column player in one cell. Another common representation is:

$$
A=\left(\begin{array}{ll}
3 & 0 \\
5 & 1
\end{array}\right), B=\left(\begin{array}{ll}
3 & 5 \\
0 & 1
\end{array}\right) .
$$

Matrix $A$ defines the payoffs for the row player, while matrix $B$ defines the payoffs for the column player. As the game is symmetric, we have $B=A^{\top}$. Let $x=\left(\begin{array}{ll}0 & 1\end{array}\right)^{\top}$ be a population of only defectors. A mutant $y \neq x$ is a strategy that includes a positive fraction $\delta \in(0,1]$ playing "cooperate", thus we define $y=\left(\begin{array}{ll}\delta & 1-\delta\end{array}\right)^{\top}$. The fitness of 
$x$ against the population mixture $\epsilon y+(1-\epsilon) x$ is computed as follows:

$$
\begin{aligned}
f(x, \epsilon y+(1-\epsilon) x) & =x^{\top} A(\epsilon y+(1-\epsilon) x) \\
& =\left(\begin{array}{ll}
0 & 1
\end{array}\right)\left(\begin{array}{ll}
3 & 0 \\
5 & 1
\end{array}\right)\left(\begin{array}{c}
\epsilon \delta \\
1-\epsilon \delta
\end{array}\right) \\
& =5 \epsilon \delta+1(1-\epsilon \delta) \\
& =4 \epsilon \delta+1 .
\end{aligned}
$$

The fitness for the mutant $y$ against the population mix is computed accordingly:

$$
f(y, \epsilon y+(1-\epsilon) x)=3 \epsilon \delta
$$

For all $\epsilon$ and any mutant $y$ with $\delta \in(0,1]$, we have $4 \epsilon \delta+1>3 \epsilon \delta$ and thus "defect" is an evolutionarily stable strategy. In this example, there is no restriction on the population share of the mutant, "defect" is an ESS for all $\bar{\epsilon}_{y} \in(0,1)$. It directly follows that "defect" is the single ESS in the prisoners' dilemma. Furthermore, we recall that "defect" is also the unique Nash equilibrium in this game. Although both solution concepts predict the same outcome in this example, in general, the set of ESS is a refinement of the set of Nash equilibria in any game. Maynard Smith (1974) gives an alternative definition of evolutionary stability that highlights this relation:

Definition 8. A strategy $x$ is an evolutionarily stable strategy (ESS) iff $\forall y \neq x$ :

$$
\begin{aligned}
& \text { I. } f(x, x)>f(y, x) \text { or } \\
& \text { 2. } f(x, x)=f(y, x) \text { and } f(y, y)<f(x, y) \text {. }
\end{aligned}
$$

Any mutant must not perform better against an evolutionarily stable strategy than the evolutionarily stable strategy does against itself. Furthermore, in case of equal fitness, the evolutionarily stable strategy must perform better against the mutant than the mutant does against itself. Condition I states that an ESS is a symmetric Nash equilibrium, that is $x$ is a best reply against itself.

\subsection{Replicator Dynamics}

Maynard Smith (1974) only defines a static solution concept that does not model the dynamic evolution of the population explicitly. Taylor and Jonker (I978) introduce the replicator dynamics to provide a dynamic model of evolution based on ordinary differential equations. The authors assume a population of individuals, each of a certain phenotype using the same pure strategy throughout their lifetime. The population mix evolves over time according to the reproduction rates of strategies under exponential 
growth or decay. Let us denote the number of individuals playing strategy $i$ by $n_{i}$ and the population size by $N=\sum_{1}^{k} n_{i}$. The state of the population is then $x=\left(x_{1} \cdots x_{k}\right)$, where $x_{i}=n_{i} / N$. Let us assume each individual of type $i$ gives rise to $f_{i}$ individuals of the same type per unit time. Given this exponential growth model, the number of $i$-strategy individuals changes according to $\frac{\partial n_{i}}{\partial t}=f_{i} n_{i}$ and the total population size according to $\frac{\partial N}{\partial t}=\left(\sum_{1}^{k} x_{i} f_{i}\right) N=\bar{f} N$, where $\bar{f}$ is the average growth rate of the population. We can now compute the rate of change of the population share $x_{i}$ as:

$$
\begin{aligned}
\frac{\partial x_{i}}{\partial t}=\frac{\partial}{\partial t}\left(\frac{n_{i}}{N}\right) & =\frac{f_{i} n_{i} N-n_{i} \bar{f} N}{N^{2}} \\
& =\frac{f_{i} n_{i}-n_{i} \bar{f}}{N} \\
& =\frac{n_{i}\left(f_{i}-\bar{f}\right)}{N} \\
& =x_{i}\left(f_{i}-\bar{f}\right) .
\end{aligned}
$$

As notation suggests, the exponential growth rate is estimated by the fitness function $f$ of the population game. We thus rewrite the dynamical system in 3.I as follows:

$$
\frac{\partial x_{i}}{\partial t}=x_{i}\left[f\left(e_{i}, x\right)-f(x, x)\right] \text {, }
$$

where $f(x, x)$ is the average payoff of an individual in the population $x$. Let us now consider a population playing the prisoners' dilemma with payoff matrix:

$$
A=\left(\begin{array}{ll}
3 & 0 \\
5 & 1
\end{array}\right) .
$$

An individual playing the strategy $i=1$, i.e. "cooperate", on average encounters $x_{1}$ individuals also "cooperating" and $x_{2}$ individuals "defecting". This means that the average fitness of an individual playing "cooperate" is $f\left(e_{1}, x\right)=(A x)_{1}=3 x_{1}+0 x_{2}$. Similarly, the average payoff of an individual playing defect is $f\left(e_{2}, x\right)=(A x)_{2}=5 x_{1}+1 x_{2}$. In general we have $f(y, x)=y^{\top} A x$.

Definition 9. The continuous time symmetric replicator dynamics for the infinite population $x$ playing a symmetric game given by payoff matrix $A$ are defined by the following system of differential equation:

$$
\frac{\partial x_{i}}{\partial t}=x_{i}\left[(A x)_{i}-x^{\top} A x\right]
$$

An intuitive explanation of (3.3) goes as follows. The payoff matrix $A$ determines the payoff an individual receives when interacting with others. The state vector $x$ describes the frequencies of all pure strategies within the population. Success of a strategy $i$ is 


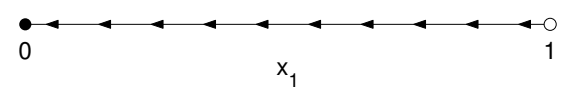

Figure 3.I: Symmetric replicator dynamics of the prisoners' dilemma. State $x_{1}=0$ corresponds to a population of defectors (asymptotically stable attractor), while $x_{1}=1$ corresponds to a population of cooperators (unstable fixed point - repeller).

measured by the difference between its current payoff $(A x)_{i}$ and the average payoff of the entire population $x^{\top} A x$. Hence, strategies that perform better than average grow in population share and those performing worse than average diminish.

Figure 3.I shows the replicator dynamics for the prisoners' dilemma. In the prisoners' dilemma we observe two fixed points at $x_{1}=0$ and $x_{1}=1$. A fixed point is either an attractor, a repeller or a saddle point. Starting from any initial interior point, the population state converges to the unique ESS at $x_{1}=0$. An interior point of the population is a state where $x_{i}>0$ for all population shares $i=1 \ldots k$. We call state $x=\left(\begin{array}{ll}0 & 1\end{array}\right)^{\top}$ an asymptotically stable attractor. The basin of attraction of this attractor consumes the entire interior space. The state $x=\left(\begin{array}{ll}1 & 0\end{array}\right)^{\top}$ is asymptotically unstable and thus called a repeller. In general, we note that any "pure" state $x=e_{i}$ for some $i$ is a fixed point under the replicator dynamics.

Another well known example, studied extensively in evolutionary game theory, is the symmetric zero-sum game rock-paper-scissors.

Example 3 (Rock-paper-scissors). Two players face each other and play by simultaneously raising and lowering one arm three times rapidly and in sync while finally resting their hands in one of three positions: rock, paper, scissors. Rock is represented by a closed fist, paper by a flat hand, while a two fingers gesture is used to symbolize a pair of scissors. Rock smashes scissors, scissors cuts paper, and paper covers rock.

The payoff matrix $A$ of the game is given below:

$$
A=\left(\begin{array}{rrr}
0 & -1 & 1 \\
1 & 0 & -1 \\
-1 & 1 & 0
\end{array}\right)
$$

Figure 3.2 shows the replicator dynamics in the game of rock-paper-scissors. The game has a unique interior Nash equilibrium strategy at $x=\left(\frac{1}{3} \frac{1}{3} \frac{1}{3}\right)^{\top}$ which is also the only interior fixed point. All trajectories starting from any other interior point are cycles. The interior fixed point and all "pure" population states are saddle points in the game of rock-paper-scissors. For further examples and the discussion of replicator dynamics of symmetric games, see Weibull (1997). 


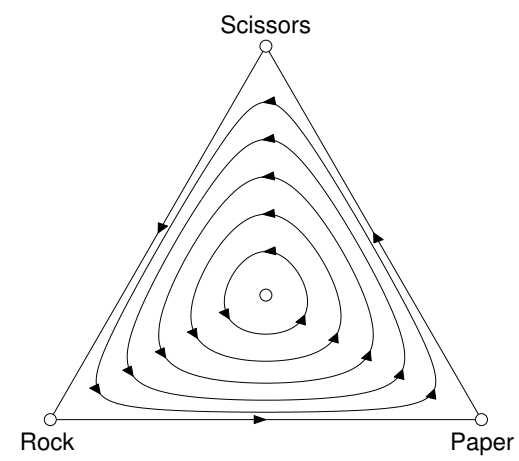

Figure 3.2: Symmetric replicator dynamics in the game of rock-paper-scissors.

\section{Asymmetric Replicator Dynamics}

The single population replicator dynamics are only applicable to symmetric games. In this section we cover asymmetric games (e.g. Example 2 - Matching pennies) and by this means introduce the asymmetric replicator dynamics or multi-population dynamics. For the sake of comprehensibility we focus on two player interactions only.

An asymmetric two-player game comprises different payoff tables for the two players and possibly different action sets. Likewise we need two separate populations to describe the dynamics. At each time step a random individual from one population interacts with a randomly matched individual from the other population. Let the game be defined by the $m \times n$ payoff matrices $A$ and $B$, where $m$ is the number of actions the row player can choose from and $n$ is the number of actions the column player can choose from. We denote the state vectors of population I and 2 by the probability distributions $x$ and $y$ respectively. Hence, the dynamics of the game are specified by a dynamical system with $m+n$ differential equations, $m$ for the replicators of $x$ and $n$ for the replicators of $y$. Equation (3.3) is adapted for the multi-population case as follows.

The fitness of an individual in population $x$ playing strategy $i$ against population $y$ is $f\left(e_{i}, y\right)=(A y)_{i}$, while the expected fitness of a random individual in $x$ against $y$ is $f(x, y)=x^{\top} A y$. The analog translation applies for population $y$.

Definition Io. The continuous time asymmetric replicator dynamics for populations $x$ and $y$ in the 2-player game $(A, B)$ are defined by the following system of differential equations:

$$
\begin{aligned}
& \frac{\partial x_{i}}{\partial t}=x_{i}\left[(A y)_{i}-x^{\top} A y\right] \\
& \frac{\partial y_{i}}{\partial t}=y_{i}\left[\left(x^{\top} B\right)_{i}-x^{\top} B y\right]
\end{aligned}
$$




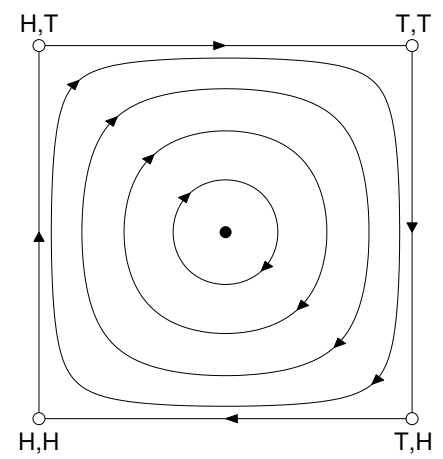

(a) Matching pennies

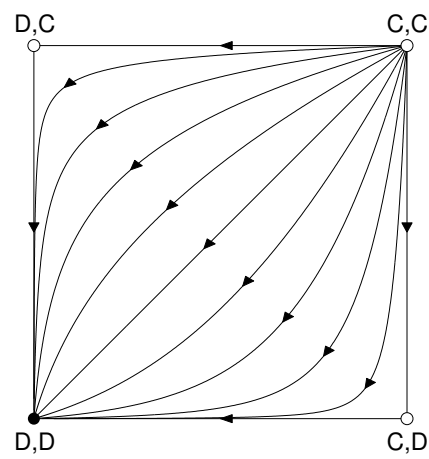

(b) Prisoners' dilemma

Figure 3.3: (a) Asymmetric replicator dynamics of the matching pennies game with pure strategies $\mathrm{H}$ (Heads) and T (Tails). (b) Asymmetric replicator dynamics of the prisoners' dilemma.

Figure 3.3(a) shows the continuous time asymmetric replicator dynamics of the game of matching pennies.

Depending on which evolutionary process is modeled, also symmetric games might be modeled using the asymmetric or multi-population replicator dynamics. For example, the evolutionary analogy of individual learning gives rise to the following interpretation. Two players play a (possibly) symmetric game. Each player is represented by a population, where the evolving population describes the dynamic change in behavior of the represented player. Therefore, population shares of pure strategies determine the current policy of a player. Both populations co-evolve and thus represent the adaptive process of the two learners pitted agains each other. Figure 3.3(b) shows the dynamics of two populations co-evolving in the prisoners' dilemma.

\subsection{Learning Dynamics}

In this section we show the mathematical relation between learning automata and the continuous time asymmetric replicator dynamics, followed by a concise survey of evolutionary models of various reinforcement learning algorithms: $Q$-learning, lenient $Q$-learning and regret minimization.

\section{Learning Automata}

We restrict the consideration to the linear reward-inaction $\left(L_{R-I}\right)$ scheme where $\beta=0$. This simplifies (2.2I) to the following update rule for action probabilities $\pi_{i}$.

$$
\pi_{t+1, i}=\pi_{t, i}+\Delta \pi_{t, i}
$$


with

$$
\Delta \pi_{t, i}=\left\{\begin{array}{cc}
\alpha R_{t}\left(1-\pi_{t, i}\right) & \text { if } i=a_{t}, \\
-\alpha R_{t} \pi_{t, i} & \text { if } i \neq a_{t} .
\end{array}\right.
$$

The policy change $\Delta \pi_{t}$ depends on the specific action $a_{t}$ selected in epoch $t$. We now assume that an agent receives an immediate reward for each possible action rather than just the feedback for a single specific action $a$. Furthermore, we assume the reward $\bar{R}_{i}$ for action $i$ is the average reward that action $i$ yields given that all other agents play according to their current policies. The action probability change in (3.7) is proportional to $\pi_{i}$ since $\pi_{i}$ determines the frequency of action $i$. Consequently, (3.8) describes the average policy change in a given epoch $t$. In the following derivation we omit $t$ for the sake of readability.

$$
\begin{aligned}
\Delta \pi_{i} & =\pi_{i}\left[\alpha \bar{R}_{i}\left(1-\pi_{i}\right)+\sum_{j \neq i}\left(-\alpha \bar{R}_{j} \pi_{j}\right)\right] \\
& =\alpha \pi_{i}\left[\bar{R}_{i}-\bar{R}_{i} \pi_{i}-\sum_{j \neq i}\left(\bar{R}_{j} \pi_{j}\right)\right] \\
& =\alpha \pi_{i}\left[\bar{R}_{i}-\sum_{j}\left(\bar{R}_{j} \pi_{j}\right)\right]
\end{aligned}
$$

If we apply (3.8) to the setting of a 2-player normal form game, the connection between automata games and replicator dynamics becomes apparent. We consider a matrix game where $A$ is the payoff for agent I (row player) and $B$ the payoff for agent 2 (column player); $\pi^{(1)}$ and $\pi^{(2)}$ are the two action probability distributions or mixed strategies for agent I and 2 respectively. Agent I receives an average payoff of $\bar{R}_{i}^{(1)}=$ $\sum_{j}\left[\operatorname{Pr}\left(a_{2}=j \mid a_{2} \sim \pi^{(2)}\right) A(i, j)\right]=\left(A \pi^{(2)}\right)_{i}$ for action $i$ against agent 2's strategy $\pi^{(2)}$. Hence, (3.8) can be rewritten as:

$$
\begin{aligned}
\Delta \pi_{i}^{(1)} & =\alpha \pi_{i}^{(1)}\left[\bar{R}_{i}^{(1)}-\sum_{j}\left(\bar{R}_{j}^{(1)} \pi_{j}^{(1)}\right)\right] \\
& =\alpha \pi_{i}^{(1)}\left[\left(A \pi^{(2)}\right)_{i}-\sum_{j}\left(\left(A \pi^{(2)}\right)_{j} \pi_{j}^{(1)}\right)\right] \\
& =\alpha \pi_{i}^{(1)}\left[\left(A \pi^{(2)}\right)_{i}-\pi^{(1)^{\top}} A \pi^{(2)}\right]
\end{aligned}
$$

Similarly, we can derive:

$$
\Delta \pi_{i}^{(2)}=\alpha \pi_{i}^{(2)}\left[\left(B \pi^{(1)}\right)_{i}-\pi^{(2)^{\top}} B \pi^{(1)}\right]
$$

for agent 2. Note that (3.9) and (3.10) in the limit $\Delta t \rightarrow 0$ correspond to the multipopulation replicator equations given in Definition Io with $x \equiv \pi^{(1)}$ and $y \equiv \pi^{(2)}$, 
scaled by the learning rate $\alpha$ :

$$
\begin{aligned}
& \frac{\partial x_{i}}{\partial t}=\alpha x_{i}\left[(A y)_{i}-x^{\top} A y\right] \\
& \frac{\partial y_{i}}{\partial t}=\alpha y_{i}\left[\left(x^{\top} B\right)_{i}-x^{\top} B y\right] .
\end{aligned}
$$

For the full proof we refer to Börgers and Sarin (1997). In particular, Börgers and Sarin prove the link between cross learning and the replicator dynamics. Cross learning is a reinforcement learning algorithm similar to the $L_{R-I}$ update scheme of learning automata. The relation between the two algorithms is explicitly shown in Tuyls (2004).

\section{Q-learning}

Tuyls et al. (2003b, 2005) derive a selection-mutation model of independent $Q$-learning in normal form games. Here, $Q$ values are interpreted as Boltzmann probabilities for action selection with time-independet temperature $\tau$, see (2.I9). The learning rate $\alpha$ is assumed to be constant. The continuous time limit of the $Q$-learning model for a 2-player normal form game with payoff tables $A$ and $B$ is captured by the following system of differential equations:

$$
\begin{aligned}
& \frac{\partial x_{i}}{\partial t}=\alpha x_{i}\left(\tau^{-1}\left[(A y)_{i}-x^{\top} A y\right]-\ln x_{i}+\sum_{j} x_{j} \ln x_{j}\right) \\
& \frac{\partial y_{i}}{\partial t}=\alpha y_{i}\left(\tau^{-1}\left[\left(x^{\top} B\right)_{i}-x^{\top} B y\right]-\ln y_{i}+\sum_{j} y_{j} \ln y_{j}\right)
\end{aligned}
$$

The first term of (3.12) is equivalent to the selection model of the asymmetric replicator dynamics scaled by factor $\tau^{-1}$. The second term describes mutation, calculated as the difference in entropy of strategy $i$ and the whole population (Tuyls et al., 2003b). Natural selection maps to exploitation in reinforcement learning (i.e. greedy action selection), while mutation provides variety and thus exploration.

The dynamical system (3.I2) predicts the expected behavior of Q-learning agents in normal form games. However, Kaisers and Tuyls (2010) show that the actual learning behavior deviates from the predicted behavior and that in case of deviation, the predicted behavior of the model is in fact more desirable. Deviations from the predicted behavior are caused by false initial estimations of the $Q$ values. When actions are initially over-estimated, the dominant action receives more updates due to being selected more often and thus quickly decreases in value, at which point the inferior action will be selected more often due to its over-estimated initial value and thus decrease in value. This process repeats and causes undesired oscillations until the action 
values are sufficiently close to the corresponding expected rewards. Based on these insights, the authors propose a modification of the learning algorithm, called FAQlearning, which is short for frequency adjusted $Q$-learning. This variation complies with the predictions of the evolutionary model for an arbitrarily large part of the policy space.

The derivation of (3.I2), see Tuyls et al. (2003b), is based on the assumption that all $Q$ values are updated in every iteration. However, the $Q$-learning update rule is only applied to the last selected action. The frequency of a particular action-value update thus depends on the policy and the employed exploration-scheme. In FAQ-learning, the update rule is modified to weight the learning rate inversely proportional to the action probability in order to match the behavior of the dynamical model:

$$
Q_{t+1}\left(a_{t}\right)=\frac{1}{\pi_{a_{t}}} \alpha\left[R_{t}+\gamma \max _{a^{\prime} \in A} Q_{t}\left(a^{\prime}\right)-Q_{t}\left(a_{t}\right)\right] .
$$

However, this version of the update rule cannot be applied numerically. As $\pi$ approaches a face of the policy simplex, the term $\pi_{a_{t}}^{-1} \alpha$ grows and eventually becomes larger than 1 for any $\alpha$ larger then the infinitesimal limit. This in turn would cause $Q$ values to be driven outside the convex hull of previously sampled rewards and thus break the learning algorithm (Kaisers and Tuyls, 20I0). The authors propose the following update rule for FAQ-learning:

$$
Q_{t+1}\left(a_{t}\right)=\min \left(\frac{\beta}{\pi_{a_{t}}}, 1\right) \alpha\left[R_{t}+\gamma \max _{a^{\prime} \in A} Q_{t}\left(a^{\prime}\right)-Q_{t}\left(a_{t}\right)\right] .
$$

The factor $\beta$ limits the area of effect of frequency adjustment. Close to the a face of the policy simplex, we have $x_{i}<\beta$ and thus (3.14) reduces to the regular $Q$-learning update rule.

\section{Lenient Q-learning}

Leniency in learning is an idea sparked by research in cooperative multi-agent learning. Reinforcement learners adapt the value of a particular action based on the expected reward received as the result of taking that action. As in a multi-agent setting the reward depends on the actions of other agents as well, the signal to noise ratio of the perceived reward is lowered. Poor rewards are not necessarily the result of an inferior action but can just as well be caused by other agents' actions. A lenient learner shows leniency to its teammates by ignoring low rewards due to actions chosen by teammates that are poor matches to the agent's current action (Panait and Luke, 2006).

Lenient multi-agent $Q$-learning collects multiple rewards for a particular stateaction pair before an update is performed. In the simplest case the $Q$ value update 
is performed using the maximum of the observed rewards. Panait et al. (2008) have derived the dynamics of lenient $Q$-learning in normal form games. Leniency changes the selection term of (3.I2). Suppose the expected rewards for action $i$ are denoted by $u_{i}$ and $w_{i}$ for players one and two respectively. It follows that the average reward of the two populations can be written as $x^{\top} u$ and $y^{\top} w$. The $Q$-learning dynamics (3.12) can be expressed in terms of $u$ and $w$ :

$$
\begin{aligned}
& \frac{\partial x_{i}}{\partial t}=\alpha x_{i}\left(\tau^{-1}\left[u_{i}-x^{\top} u\right]-\ln x_{i}+\sum_{j} x_{j} \ln x_{j}\right) \\
& \frac{\partial y_{i}}{\partial t}=\alpha y_{i}\left(\tau^{-1}\left[w_{i}-y^{\top} w\right]-\ln y_{i}+\sum_{j} y_{j} \ln y_{j}\right) .
\end{aligned}
$$

The expected reward vectors $u$ and $w$ are affected by leniency. The expected maximum payoff for action $i$ over $K$ interactions is computed as follows:

$$
\begin{aligned}
u_{i} & =\sum_{j} \frac{A_{i j} y_{j}\left[\left(\sum_{k: A_{i k} \leq A_{i j}} y_{k}\right)^{K}-\left(\sum_{k: A_{i k}<A_{i j}} y_{k}\right)^{K}\right]}{\sum_{k: A_{i k}=A_{i j}} y_{k}} \\
w_{i} & =\sum_{j} \frac{B_{j i} x_{j}\left[\left(\sum_{k: B_{k i} \leq B_{j i}} x_{k}\right)^{K}-\left(\sum_{k: B_{k i}<B_{j i}} x_{k}\right)^{K}\right]}{\sum_{k: B_{k i}=B_{j i}} x_{k}}
\end{aligned}
$$

For a discussion of lenient frequency adjusted $Q$-learning, see Bloembergen et al. (2oIob).

\section{Regret Minimization}

Klos et al. (2010) have derived the evolutionary dynamics of the polynomial weight regret minimization algorithm. Every reinforcement learning agent incurs a loss when (initially) following a suboptimal policy. In hindsight, the agent regrets not having taken the optimal action at every time step. Regret minimization adapts the agent's policy in order to minimize the expected regret.

Polynomial weight regret minimization uses full information and external regret. External regret means that the loss is computed with respect to the optimal action in hindsight. If the agent knows the value of all actions in hindsight, it is said to have full information.

The dynamics of two player polynomial weight regret minimization in normal form games are described by the following dynamical system: 


$$
\begin{aligned}
\frac{\partial x_{i}}{\partial t} & =\frac{\alpha x_{i}\left[(A y)_{i}-x^{\top} A y\right]}{1-\alpha\left[\max _{k}(A y)_{k}-x^{\top} A y\right]} \\
\frac{\partial y_{i}}{\partial t} & =\frac{\alpha y_{i}\left[\left(x^{\top} B\right)_{i}-x^{\top} B y\right]}{1-\alpha\left[\max _{k}\left(x^{\top} B\right)_{k}-x^{\top} B y\right]}
\end{aligned}
$$

\section{Learning Dynamics in 2x2 Games}

The family of two player two strategy normal form games, or 2x2 games, can be classified in three general subclasses. Suppose we have the following payoff matrices $A$ and $B$ for the row and column player respectively:

$$
A=\left(\begin{array}{ll}
a_{11} & a_{12} \\
a_{21} & a_{22}
\end{array}\right), \quad B=\left(\begin{array}{ll}
b_{11} & b_{12} \\
b_{21} & b_{22}
\end{array}\right) .
$$

The three subclasses are characterised by the payoff matrices as follows:

Class I: $\left(a_{11}-a_{21}\right)\left(a_{12}-a_{22}\right)>0$ or $\left(b_{11}-b_{12}\right)\left(b_{21}-b_{22}\right)>0$ :

Games with one pure equilibrium; at least one player has a dominated strategy.

Class 2: $\left(a_{11}-a_{21}\right)\left(a_{12}-a_{22}\right)<0$ and $\left(b_{11}-b_{12}\right)\left(b_{21}-b_{22}\right)<0$ and $\left(a_{11}-a_{21}\right)\left(b_{11}-b_{12}\right)>0$ : Games with two pure equilibria and one mixed equilibrium; neither player has a dominated strategy but both players receive maximum payoff by playing either their first or second strategy.

Class 3: $\left(a_{11}-a_{21}\right)\left(a_{12}-a_{22}\right)<0$ and $\left(b_{11}-b_{12}\right)\left(b_{21}-b_{22}\right)<0$ and $\left(a_{11}-a_{21}\right)\left(b_{11}-b_{12}\right)<0$ : Games with one mixed equilibrium; neither player has a dominated strategy and both players do not receive maximum payoff by playing either their first or second strategy.

The prisoners' dilemma falls in class C.I: Strategy "defect" is a dominant strategy for both players and thus mutual defection is the unique Nash equilibrium. Matching pennies is an example of class C.3: The unique mixed-strategy equilibrium is playing "heads" and "tails" with 0.5 probability each. We now introduce a third example that belongs to class C.2:

Example 4 (Bach or Stravinsky game). In this strategic situation the two players want to visit a concert together. They can chose between "Bach" or "Stravinsky" but no communication is allowed. The row player prefers Bach whereas the column player has a preference for Stravinsky. However, players only enjoy the event when both select the same concert. The game is also known as the battle of the sexes, with strategies "Ballet" and "Soccer". 
Table 3.I: Payoff table of the Bach or Stravinsky game.

\begin{tabular}{c|c|c|}
\multicolumn{1}{c}{} & \multicolumn{1}{c}{ Bach } & \multicolumn{1}{c}{ Stravinsky } \\
\cline { 2 - 3 } Bach & 2,1 & 0,0 \\
\cline { 2 - 3 } Stravinsky & 0,0 & 1,2 \\
\cline { 2 - 3 } & &
\end{tabular}

Table 3.I shows the payoffs for the Bach or Stravinsky game. The two pure equilibria are realized when the two players coordinate, i.e. both play strategy "Bach" or both play "Stravinsky". The players are in a mixed equilibrium if both play their preferred action with $\frac{1}{3}$ probability. Figure 3.4 shows the replicator dynamics of the game of Bach or Stravinsky.

We now illustrate the learning behavior of learning automata for this selection of $2 \times 2$ games and compare results to the prediction of the corresponding evolutionary model. All automata games use the linear reward-inaction $\left(L_{R-I}\right)$ scheme with a small learning rate $\alpha=0.005$. Initial policies for automata and replicator trajectory plots are generated randomly.

A straightforward way of displaying evolving policies are trajectory plots. In a $2 \times 2$ matrix game the action probabilities for first and second action add up to one, i.e., $\pi_{2}^{(1)}=1-\pi_{1}^{(1)}$ and $\pi_{2}^{(2)}=1-\pi_{1}^{(2)}$. Therefore the strategy profile $\pi=\left\langle\pi^{(1)}, \pi^{(2)}\right\rangle$ can be reduced to the pair $\left\langle\pi_{1}^{(1)}, \pi_{1}^{(2)}\right\rangle$ without loss of information. The trajectory of this pair is recorded during one or multiple runs and plotted in a two dimensional space. To indicate the direction and speed of convergence, gray scales are used for the trajectory plots. With increasing number of epochs $t$ the brightness of the trajectory changes from light to dark. In addition to the trajectory of a pair of learners we can also compute the trajectory of the reduced state $\left\langle x_{1}, y_{1}\right\rangle$ of the dynamical system (3.II).

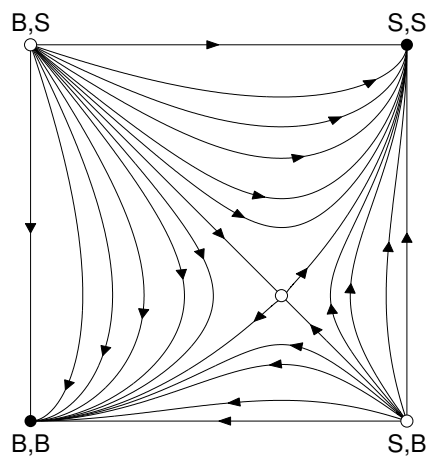

Figure 3.4: Asymmetric replicator dynamics of the Bach or Stravinsky game. The two strategies are indicated by B (Bach) and S (Stravinsky). 

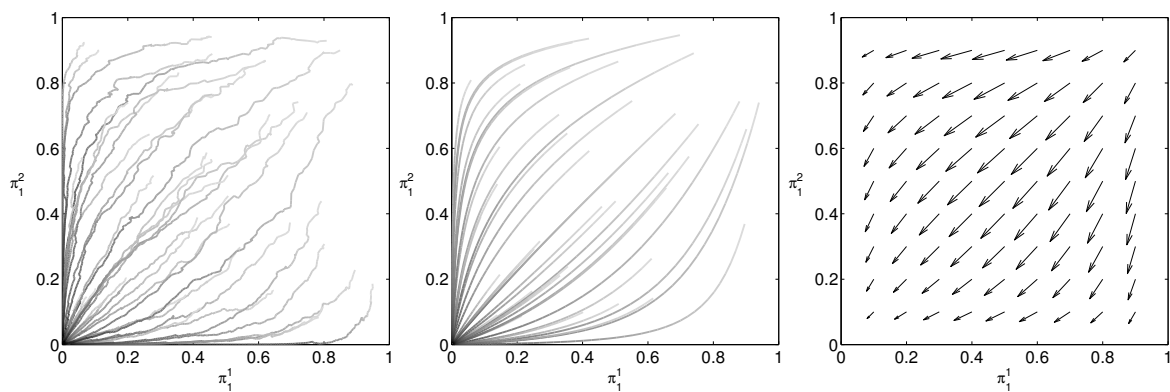

Figure 3.5: Dynamics in the single state prisoners' dilemma. From left to right: trajectory plot of the automata game, trajectory plot for the corresponding dynamics and vector field plot.

Another visual method to analyze learning dynamics are vector field plots. Vector field plots are often used to examine dynamic systems and are an excellent tool to identify basins of attraction. Arrows starting at regular grid points indicate the direction and velocity of movement through the state space of the dynamic system. For grid points $\left\langle x_{1}, y_{1}\right\rangle \in[0,1] \times[0,1]$ over the reduced strategy profile of a $2 \times 2$ game the vector field is denoted by:

$$
\langle v, u\rangle=\frac{\partial}{\partial t}\left(\left\langle x_{1}, y_{1}\right\rangle\right)=\left\langle\frac{\partial x_{1}}{\partial t}, \frac{\partial y_{1}}{\partial t}\right\rangle
$$

where $\frac{\partial x_{1}}{\partial t}$ and $\frac{\partial y_{1}}{\partial}$ are the asymmetric-population replicator equations (3.II). The vectors are displayted by arrows pointing in the direction of $\langle v, u\rangle$, while the velocity $\|\langle v, u\rangle\|$ is normalized and indicated by the length of an arrow originating from that point in the state space.

Figure 3.5 shows the dynamics in the prisoners' dilemma. The automata game as well as the corresponding dynamics show similar evolution toward the equilibrium strategy of mutual defection. Action probabilities are plotted for action I (in this case cooperate); $\mathrm{x}$ - and $\mathrm{y}$-axis correspond to the action of player $\mathrm{I}$ and 2 respectively. Hence, the Nash equilibrium point is located at the origin $(0,0)$.

Figure 3.6 illustrates the dynamics in the Bach or Stravinsky game. Again, automata games and corresponding dynamics show similar policy evolution and eventually converge to either one of the pure equilibrium points $(0,0)$ or $(1,1)$. The mixed Nash equilibrium is a strange attractor, meaning that policy trajectories will initially move toward this point but fail to converge and eventually become subjected to the influence of either one of the two strong attractors.

The Matching Pennies game shows cyclic behavior for automata games and corresponding dynamics alike. The dynamics are plotted in Figure 3.7. Bloembergen et al. (20I0a) present an extensive comparative analysis of learning dynamics in self-play and mixed-play in 2x2 normal-form games. 

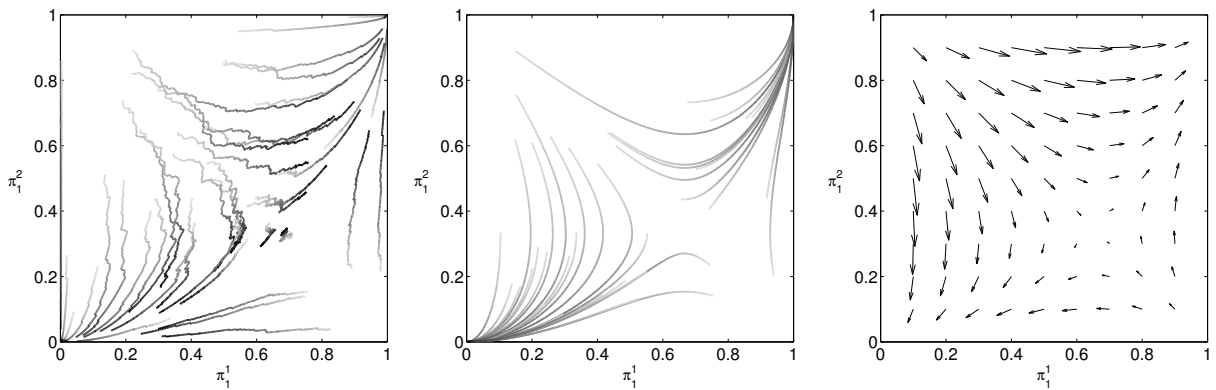

Figure 3.6: Dynamics in the Bach or Stravinsky game. From left to right: trajectory plot of the automata game, trajectory plot for the corresponding dynamics and vector field plot.
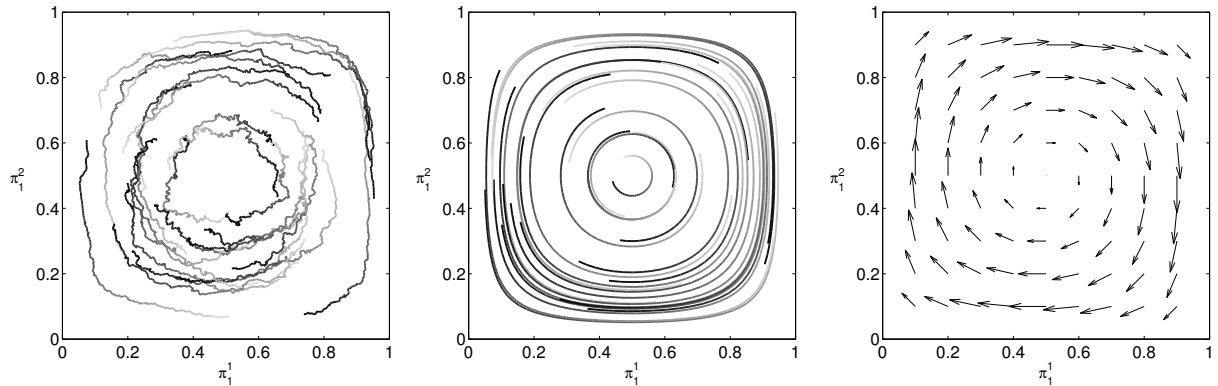

Figure 3.7: Dynamics in the matching pennies game. From left to right: trajectory plot of the automata game, trajectory plot for the corresponding dynamics and vector field plot.

\section{Generalizing beyond two-player games}

In previous sections we have introduced a variety of modifications of the replicator dynamics mapping to various reinforcement learners in two-player normal form games. We now give a more general formulation for $n$ populations (or players).

Let $X=\left\langle x^{(1)}, \ldots, x^{(n)}\right\rangle$ be the full system state, where $x^{(j)}$ is the state of population $j$. The fitness function for population $j$ is denoted by $f^{(j)}(\cdot, X)$. The average reward $\Phi^{(j)}$ of population $j$ is computed as follows:

$$
\Phi^{(j)}(X)=\sum_{i} x_{i}^{(j)} f^{(j)}\left(e_{i}, X\right)
$$

The general $n$-population continuous time replicator dynamics are written as the following dynamical system: 


$$
\frac{\partial x_{i}^{(j)}}{\partial t}=x_{i}^{(j)}\left[f^{(j)}\left(e_{i}, X\right)-\Phi^{(j)}(X)\right], \quad j=1, \ldots, n
$$

\subsection{Dynamics of a Population of Learners}

We have seen that population games offer different ways of interpretation. On the one hand, a population is a collection of individuals, each representing a certain phenotype, i.e., a pure strategy. An individual never changes its phenotype during the course of its lifetime. Individuals are randomly matched and play the game according to their predetermined phenotypes; subsequently, phenotypes replicate according to the realized payoffs. Thus phenotypes compete with each other - fitter strategies prevail while inferior strategies eventually die out.

On the other hand, a population might also represent the behavior of a particular agent. The population shares reflect the current preferences over different strategies and thus defines the agent's policy. The asymmetric replicator dynamics provide a model for two learning agents pitted against each other and thus, two populations co-evolving. In particular, we have also studied the asymmetric population dynamics in games with symmetric payoff tables which is motivated by the same interpretation.

We will now derive the dynamics of a large population of learners which are randomly matched to play a symmetric game. This is a combination of both former interpretations: a large population of individuals randomly engage in an interaction, where each individual is an adaptive agent maintaining a population of possible strategies encoding its policy.

For a population of $n$ agents, let $X=\left\langle x^{(1)}, \ldots, x^{(n)}\right\rangle$ be the full system state with $x^{(j)} \in \Delta^{k-1}$ for all $j$. Furthermore, let us define the overall average population share of strategy $i$ as:

$$
\bar{x}_{i}=\frac{1}{n} \sum_{j=1}^{n} x_{i}^{(j)}
$$

For a symmetric game the same fitness function applies to all agents, thus:

$$
\forall_{j}: f^{(j)}(\cdot, \cdot)=f(\cdot, \cdot)
$$

Suppose the game has the payoff matrix $A$. For some population $p \in \Delta^{k-1}$ we have the following fitness in the current state $X$ :

$$
f(p, X)=p^{\top} A \bar{x}
$$


Therefore with (3.20) and (3.2I), system (3.19) can be rewritten as:

$$
\begin{aligned}
\frac{\partial x_{i}^{(j)}}{\partial t} & =x_{i}^{(j)}\left[f\left(e_{i}, X\right)-\sum_{i} x_{i}^{(j)} f\left(e_{i}, X\right)\right] \\
& =x_{i}^{(j)}\left[(A \bar{x})_{i}-x^{(j)^{\top}} A \bar{x}\right] .
\end{aligned}
$$

We can now derive the change in overall population share $\bar{x}_{i}$ as follows:

$$
\begin{aligned}
\frac{\partial \bar{x}_{i}}{\partial t} & =\frac{\partial\left(\frac{1}{n} \sum_{j=1}^{n} x_{i}^{(j)}\right)}{\partial t} \\
& =\frac{1}{n} \sum_{j=1}^{n} \frac{\partial x_{i}^{(j)}}{\partial t} \\
& =\frac{1}{n} \sum_{j=1}^{n} x_{i}^{(j)}\left[(A \bar{x})_{i}-x^{(j)^{\top}} A \bar{x}\right] \\
& =\frac{1}{n} \sum_{j=1}^{n} x_{i}^{(j)}(A \bar{x})_{i}-\frac{1}{n} \sum_{j=1}^{n} x_{i}^{(j)}\left(x^{(j)^{\top}} A \bar{x}\right) \\
& =\bar{x}_{i}(A \bar{x})_{i}-\sum_{j=1}^{n} x_{i}^{(j)}\left(x^{(j)^{\top}} A \bar{x}\right) .
\end{aligned}
$$

We see that the second term in (3.23) is dependent on the particle population shares of populations $x^{(j)}$ and cannot be abstracted in terms of the overall population shares $\bar{x}$. Although the system assumes a large number of learners that engage in interactions at random, the information encoded in the behavior of each learner is important to the learning process.

Alternatively to the assumption that players are randomly matched with equal probability, we can assume that the population average $\bar{x}$ assigns a weight $\omega_{j}$ to each population $x^{(j)}$ :

$$
\bar{x}=\sum_{j=1}^{n} \omega_{j} x^{(j)} .
$$

In the previous approach, the weights are $\omega_{j}=\frac{1}{n}$ for all $j$. Now, populations that have a higher weight $\omega_{j}$ are more likely to be selected to play the game. This raises the question of how to assign weights to the populations. In the replicator dynamics model, each pure strategy $i$ is represented by individuals of a the same fixed phenotype $i$. Therefore, the population share of $i$ determines the probability of strategy $i$ being played in a random interaction. 
Let us assume the weights $\omega$ are dynamic and the rate of change is defined similar to the replicator equations:

$$
\frac{\partial \omega_{j}}{\partial t}=\omega_{j}\left[x^{(j)^{\top}} A \bar{x}-\bar{x}^{\top} A \bar{x}\right] .
$$

Each population has a weight that changes at the rate of $x^{(j)^{\top}} A \bar{x}-\bar{x}^{\top} A \bar{x}$ which is the average fitness of population $x^{(j)}$ compared to the overall average fitness $\bar{x}^{\top} A \bar{x}$. We can now derive the rate of change of the overall average population share $\bar{x}_{i}$ for strategy $i$ as follows:

$$
\begin{aligned}
\frac{\partial \bar{x}_{i}}{\partial t} & =\frac{\partial\left(\sum_{j=1}^{n} \omega_{j} x_{i}^{(j)}\right)}{\partial t}=\sum_{j=1}^{n} \frac{\partial\left(\omega_{j} x_{i}^{(j)}\right)}{\partial t} \\
& =\sum_{j=1}^{n}\left(x_{i}^{(j)} \omega_{j}\left[x^{(j)^{\top}} A \bar{x}-\bar{x}^{\top} A \bar{x}\right]+\omega_{j} x_{i}^{(j)}\left[(A \bar{x})_{i}-x^{\left.\left.(j)^{\top} A \bar{x}\right]\right)}\right.\right. \\
& =\sum_{j=1}^{n}\left(x_{i}^{(j)} \omega_{j}\left[x^{(j)^{\top}} A \bar{x}-\bar{x}^{\top} A \bar{x}+(A \bar{x})_{i}-x^{(j)^{\top}} A \bar{x}\right]\right) \\
& =\left(\sum_{j=1}^{n}\left(x_{i}^{(j)} \omega_{j}\right)\right)\left[(A \bar{x})_{i}-\bar{x}^{\top} A \bar{x}\right] \\
& =\bar{x}_{i}\left[(A \bar{x})_{i}-\bar{x}^{\top} A \bar{x}\right] .
\end{aligned}
$$

For dynamic weights $\omega$ that change according to (3.24), the overall population average $\bar{x}$ as given in (3.25) moves independent of the behavior of individual learners.

This is of particular interest as techniques like leniency, see Section 3.3, or "Win or Learn Fast", see Section 2.6, change the learning rate to accommodate for the fact that low rewards are often caused by poor behavior of other agents and not necessarily by an inferior action choice. Similarly, if the frequency of interactions between learners is dynamically changed according to their performance, poor behaving agents have less effect on the evolution of the population.

\subsection{Summary}

This chapter has aimed to highlight the benefits of an evolutionary game theoretic approach to multi-agent learning.

For all three subclasses of $2 \times 2$ matrix games we observe similar dynamics for automata games and replicators. This affirms the previously made statement that replicator dynamics indeed relate to learning automata as mathematically indicated in Sec- 
tion 3.3. Exploiting this link between reinforcement learning and evolutionary game theory is beneficial for several reasons (Tuyls, 2004). The majority of contemporary reinforcement learning algorithms are blackbox models, i.e. it is not explicit why we observe certain outcomes. Hence, it is difficult to predict the learning processes and this becomes even more intricate once the learning task increases in complexity as multiple agents are involved. Making the link between multi-agent learning and evolutionary game theory explicit adds a new view point and therefore facilitates the understanding of learning dynamics.

The same line of argumentation applies to parameter tuning in learning algorithms. Many learning algorithms depend on a set of parameters and show only desired behavior for well chosen configurations. Analyzing the learning dynamics formally helps to determine parameter configurations before learners are actually deployed in the task domain.

Finally, the evolutionary game theoretic approach to multi-agent learning allows to inverse the problem and design learning algorithms that show predefined learning dynamics (Tuyls, 2004; Panait and Tuyls, 2007; Kaisers and Tuyls, 2010; Hennes et al., 2010). 


\section{4 Stochastic Game Dynamics}

The previous chapter illustrates that multi-agent learning can be modeled by the replicator dynamics and that exploiting this link offers a variety of benefits. The main limitation of this approach lies in its restriction to one subclass of dynamic games: stateless repeated games. Even though real-life tasks might be modeled statelessly, the majority of such problems naturally relates to multi-state situations. Vrancx et al. (2008) make the first attempt to extend replicator dynamics to multi-state games. More precisely, the authors combine replicator dynamics and piecewise dynamics, called piecewise replicator dynamics, to model the learning behavior of agents in stochastic games.

Piecewise models are a methodology in the area of dynamical systems theory. The core concept is to partition the state space of a dynamical system into cells. The behavior of a dynamical system can then be described as the state vector movement through this collection of cells. Dynamics within each cell are determined by the presence of an attractor or repeller. Piecewise linear systems make the assumption that each cell is reigned by a specific attractor and that the induced dynamics can be approximated linearly. Piecewise linear systems are especially suited to be analyzed mathematically due to their reduced complexity. However, they are often still capable of demonstrating the essential qualitative features of many non-linear dynamical systems. Applications include for example the analysis of regulatory networks in the area of biology and genomics (de Jong et al., 200I).

In this chapter, we show how the approach of piecewise models can be translated to replicator dynamics by developing a rigorous formalization of the work by Vrancx et al. (2008). Furthermore, we identify shortcomings of piecewise modeling in the domain of replicator dynamics and subsequently propose a new set of stochastic game dynamics, named state-coupled replicator dynamics. Grounded on the formalization of piecewise replicators, the new model describes the direct coupling between states and thus overcomes the problem of anomalies induced by approximation.

In Section 4.I we formally introduce piecewise replicator dynamics. Section $\mathbf{4 . 2}$ presents the new state-coupled model and delivers a comparative study of piecewise 
and state-coupled replicator dynamics and networked learning automata. In Section 4.3 we derive symmetric state-coupled replicator dynamics and Section 4.4 introduces mutation to the multi-state population model.

\section{I Piecewise Replicator Dynamics}

Multi-state learning dynamics are significantly more complex than single state dynamics. Agents maintain a policy for each state and optimizing these policies is a very high dimensional problem. Piecewise replicator dynamics analyze the dynamics per state in order to cope with this problem (Vrancx et al., 2008). For each state of a stochastic game a so called average reward game is computed. An average reward game determines the expected reward for each joint action in a given state, assuming fixed strategies in all other states. This method projects the limit average reward of a stochastic game onto a stateless normal-form game, which then can be analyzed using the continuous time asymmetric replicator dynamics (see Section 3.2).

In general we can not assume that strategies are fixed in all but one state. Agents adapt their policies in all states in parallel and therefore the average reward game along with the linked replicator dynamics are changing as well. The core idea of piecewise replicator dynamics is to partition the strategy space into cells, where each cell corresponds to a set of attractors in the average reward game. This approach is based on the methodology of piecewise dynamical systems.

In piecewise dynamical systems theory, the state vector of a system eventually enters an area of attraction or repulsion and becomes subject to the influence of this attractor or repeller. In case of piecewise replicator dynamics the state vector is an element of the strategy space and attractors resemble equilibrium points in the average reward game. It is assumed that the dynamics in each cell are reigned by a set of equilibria and therefore we can qualitatively describe the dynamics of each cell by a set of replicator equations.

We can use this approach to model learning dynamics in stochastic games as follows. For each state of a stochastic game we derive the average reward game and consider the strategy space over all joint actions for all other states. This strategy space is then partitioned into cells, where each cell corresponds to a set of equilibrium points in the average reward game. We sample the strategy space of each cell and compute the corresponding limit average reward for each joint action, eventually leading to a set of replicator equations for each cell. More precisely, each state features a number of cells, each related to a set of replicator dynamics. For each state, a single cell is active and the associated replicator equations determine the dynamics in that state, while the active cell of a particular state is exclusively determined by the strategies in all other states. Strategy changes occur in all states in parallel and hence mutually induce cell switching. 


\section{Average Reward Game}

Let us assume for a repeated $n$-player game, the objective of player $i$ at stage $t=t_{0}$ is to maximize the limit average reward $\bar{R}^{(i)}$ given by:

$$
\bar{R}^{(i)}=\liminf _{T \rightarrow \infty} \frac{1}{T-t_{0}} \mathbb{E}\left[\sum_{t=t_{0}}^{T-1} R^{(i)}\left(s_{t}, a_{t}\right)\right],
$$

where $R^{(i)}\left(s_{t}, a_{t}\right)$ is the immediate payoff to player $i$ for joint action $a_{t}$ in state $s_{t}$. The scope of this thesis is restricted to irreducible stochastic games where the sequence of game states $s_{0}, s_{1}, s_{2}, \ldots, s_{T}$ is ergodic (see Section 2.5), i.e., each state $s$ occurs infinitely often with probability 1 . Hence, there exists a stationary distribution $\chi \in \Delta(S)$ over all states, where fraction $\chi_{s}$ determines the frequency of state $s$ in the sequence. Therefore, we can rewrite (4.I) as

$$
\bar{R}^{(i)}=\sum_{s \in S} \chi_{s} \mathbb{E}\left[R^{(i)}(s) \mid \pi\right]
$$

where $\mathbb{E}\left[R^{(i)}(s) \mid \pi\right]$ is the expected payoff of player $i$ in state $s$ given that players play according to the strategy profile $\pi=\left\langle\pi^{(1)}, \ldots, \pi^{(n)}\right\rangle$.

In piecewise replicator dynamics, states are analyzed separately to cope with the high dimensionality. Thus, let us assume the game is in state $s$ at stage $t_{0}$ and players play a given joint action $a$ in $s$ and fixed strategies $\pi\left(s^{\prime}\right)$ in all states but $s$. Then the limit average reward becomes

$$
\begin{aligned}
\bar{R}^{(i)}(s, a)= & \sum_{s^{\prime} \in S-\{s\}}\left[\chi_{s^{\prime}} \mathbb{E}\left[R^{(i)}\left(s^{\prime}\right) \mid \pi\right]\right]+\chi_{s} R^{(i)}(s, a) \\
= & \sum_{s^{\prime} \in S-\{s\}}\left[\chi_{s^{\prime}} \sum_{a^{\prime} \in \prod_{i=1}^{n} A^{(i)}\left(s^{\prime}\right)}\left(R^{(i)}\left(s^{\prime}, a^{\prime}\right) \prod_{i=1}^{n} \pi_{a^{\prime(i)}}^{(i)}\left(s^{\prime}\right)\right)\right] \\
& +\chi_{s} R^{(i)}(s, a) .
\end{aligned}
$$

An intuitive explanation of (4.2) goes as follows. At each stage players consider the infinite horizon of payoffs under current strategies. We untangle the current state $s$ from all other states $s^{\prime} \neq s$ and the limit average payoff $\bar{R}^{(i)}$ becomes the sum of the immediate payoff for joint action $a$ in state $s$ and the expected payoffs in all other states. Payoffs are weighted by the frequency of corresponding state occurrences. Thus, if players invariably play joint action $a$ every time the game is in state $s$ and their fixed strategies $\pi\left(s^{\prime}\right)$ for all other states, the limit average reward for $T \rightarrow \infty$ is expressed by (4.2). 
Since a specific joint action $a$ is played in state $s$, the stationary distribution $\chi$ depends on $s$ and $a$ as well. A formal definition is given below.

Definition II. For a stochastic game $G=\left\langle n, S, A^{(1)}, \ldots, A^{(n)}, R^{(1)}, \ldots, R^{(n)}, T\right\rangle$ and strategy profile $\pi=\left\langle\pi^{(1)}, \ldots, \pi^{(n)}\right\rangle$ where $S$ itself is the only ergodic set in $S=\left\{s_{1}, \ldots, s_{k}\right\}$, we say $\chi(s, a)$ is a stationary distribution if and only if:

$$
\sum_{z \in S} \chi_{z}(s, a)=1
$$

and

$$
\begin{aligned}
\chi_{z}(s, a)= & \sum_{s^{\prime} \in S-\{s\}}\left[\chi_{s^{\prime}}(s, a) \sum_{a^{\prime} \in \prod_{i=1}^{n} A^{(i)}\left(s^{\prime}\right)}\left(T_{z}\left(s^{\prime}, a^{\prime}\right) \prod_{i=1}^{n} \pi_{a^{\prime}(i)}^{i}\left(s^{\prime}\right)\right)\right] \\
& +\chi_{s}(s, a) T_{z}(s, a) .
\end{aligned}
$$

Based on this notion of stationary distribution and limit average reward (4.2) we can define the average reward game as follows:

Definition I2. For a stochastic game $G=\left\langle n, S, A^{(1)}, \ldots, A^{(n)}, R^{(1)}, \ldots, R^{(n)}, T\right\rangle$ and strategy profile $\pi=\left\langle\pi^{(1)}, \ldots, \pi^{(n)}\right\rangle$ where $S$ itself is the only ergodic set in $S=\left\{s_{1}, \ldots, s_{k}\right\}$, we define the average reward game for some state $s \in S$ as the normal-form game:

$$
\bar{G}\left(s,\left\langle\pi^{(1)}, \ldots, \pi^{(n)}\right\rangle\right)=\left\langle n, A^{(1)}(s), \ldots, A^{(n)}(s), R^{(1)}, \ldots, R^{(n)}, \pi^{1}(s), \ldots, \pi^{n}(s)\right\rangle,
$$

where each player i plays a fixed strategy $\pi^{(i)}\left(s^{\prime}\right)$ in all states $s^{\prime} \neq s$. The payoff function $\bar{R}$ is given by:

$$
\begin{aligned}
\bar{R}^{(i)}(s, a)= & \sum_{s^{\prime} \in S-\{s\}}\left[\chi_{s^{\prime}} \sum_{a^{\prime} \in \prod_{i=1}^{n} A^{(i)}\left(s^{\prime}\right)}\left(R^{(i)}\left(s^{\prime}, a^{\prime}\right) \prod_{i=1}^{n} \pi_{a^{\prime(i)}}^{i}\left(s^{\prime}\right)\right)\right] \\
& +\chi_{s} R^{(i)}(s, a) .
\end{aligned}
$$

This formalization of the average reward game has laid the basis for the definition and analysis of pure equilibrium cells.

\section{Equilibrium Cells}

The average reward game projects the limit average reward for a given state onto a stateless normal-form game. This projection depends on the fixed strategies in all other states. In this section we explain how this strategy space can be partitioned into discrete cells, each corresponding to a set of equilibrium points of the average reward game. 
First, we introduce the concept of a pure equilibrium cell. Such a cell is a subset of all strategy profiles under which a given joint action specifies a pure equilibrium in the average reward game. In a Nash equilibrium situation, no player can improve its payoff by unilateral deviation from its own strategy $\pi^{(i)}$. In the context of an average reward game, all strategies including $\pi^{(i)}\left(s^{\prime}\right)$ are fixed for all states $s^{\prime} \neq s$. Therefore, the payoff $\bar{R}^{(i)}(s, a)$, see (4.2), depends only on the joint action $a$ in state $s$. Hence, the equilibrium constraint translates to:

$$
\forall_{i} \forall_{a^{\prime} \in A^{(i)}(s)}: \bar{R}^{(i)}(s, a) \geq \bar{R}^{(i)}\left(s,\left\langle a^{(i)}, \ldots, a^{(i-1)}, a^{\prime}, a^{(i+1)}, \ldots, a^{(n)}\right\rangle\right)
$$

Consequently, this leads to the following definition of pure equilibrium cells:

Definition I3. We call $C(s, a)$ a pure equilibrium cell of a stochastic game $G$ if and only if $C(s, a)$ is the subset of all strategy profiles under which the following condition holds:

$$
\forall_{i} \forall_{a^{\prime}}: \bar{R}^{(i)}(s, a) \geq \bar{R}^{(i)}\left(s, a^{\prime}\right)
$$

where $\bar{R}$ is the payoff function of the average reward game $\bar{G}\left(s,\left\langle\pi^{(1)}, \ldots, \pi^{(n)}\right\rangle\right)$; joint actions $a$ and $a^{\prime}$ satisfy $\forall_{j \neq i}: a^{(j)}=a^{(j)}$. Thus, $a$ is a pure equilibrium in state $s$ for all strategy profiles $\pi \in C(s, a)$.

Note that $\bar{R}$ is independent of the players' strategies in $s$. Hence, we can express the cell boundaries in state $s=s_{i}$ as a function of the profile:

$$
\left\langle\pi\left(s_{1}\right), \ldots, \pi\left(s_{i-1}\right), \pi\left(s_{i+1}\right), \ldots, \pi\left(s_{k}\right)\right\rangle
$$

i.e., players' strategies in all but state $s$. However, pure equilibrium cells might very well overlap for certain areas of this strategy space (Vrancx et al., 2008). Therefore, we consider all possible combinations of equilibrium points within one state and partition the strategy space of all other states into corresponding discrete cells.

\section{Strategy Space Sampling}

The partitioned strategy space is sampled in order to compute particular average reward game payoffs that in turn are used to obtain the set of replicator equations. For each state and each discrete cell, the corresponding strategy space is scanned using an equally spaced grid. Each grid point defines a specific joint strategy of all states but the one under consideration. Average reward game payoffs are averaged over all grid points and the resulting payoffs are embedded in the set of replicator equations $R D_{c_{s}}$ for a specified cell $c$ in state $s$. 


\section{Definition}

Now we link the concepts of average reward game, pure equilibrium cells and strategy space sampling and give a definition of piecewise replicator dynamics.

For each state $s=s_{i}$ the strategy space in all remaining states is partitioned into discrete cells. Each cell $c_{s} \subset A\left(s_{1}\right) \times \ldots \times A\left(s_{i-1}\right) \times A\left(s_{i+1}\right) \times \ldots \times A\left(s_{k}\right)$ refers to some combination of pure equilibria. Note that each $A(s)$ is the set of joint actions $A(s)=A^{(1)} \times \ldots \times A^{(n)}$. This combination might as well resemble only a single equilibrium point or the empty set, i.e., no pure equilibrium in the average reward game. As explained in the previous section, the strategy subspace of each cell is sampled. As a result, we obtain payoff matrices that in turn lead to a set of replicator equations $R D_{c_{s}}$ for each cell. However, the limiting distribution over states under the strategy profile $\pi$ has to be factored into the system. This means that different strategies result in situations where certain states are visited more frequently than others. Since we model each cell in each state with a separate set of replicator dynamics, we need to scale the change of $\pi(s)$ with frequency $\chi_{s}$. The frequency $\chi_{s}$ determines the expected fraction of upcoming stages played in state $s$. Finally, we recall from Section 3.3 that a strategy profile can be captured by a system of populations $X=\left\langle x^{(1)}, \ldots, x^{(n)}\right\rangle$ where each population represents the mixed strategy $\pi^{(i)}$ of corresponding player $i$. In piecewise replicator dynamics we have one such system for each state of the game, i.e., $X(s)=\left\langle x^{(1)}(s), \ldots, x^{(n)}(s)\right\rangle$.

Definition I4. The piecewise replicator dynamics are defined by the following system of differential equations:

$$
\frac{\partial X(s)}{\partial t}=R D_{c_{s}}(X(s)) \chi_{s}
$$

where $c_{s}$ is the active cell in state $s$ and $R D_{c_{s}}$ is the set of replicator equations that reign in cell $c_{s}$. Furthermore, $\chi$ is the stationary distribution over all states $S$ under strategy profile $\pi$, with $\sum_{s \in S} \chi_{s}(\pi)=1$ and

$$
\chi_{s}(\pi)=\sum_{z \in S}\left[\chi_{z}(\pi) \sum_{a \in \prod_{i=1}^{n} A^{i}(s)}\left(T_{s}(z, a) \prod_{i=1}^{n} x_{a^{(i)}}^{(i)}(s)\right)\right] .
$$

Note that $\chi_{s}$ is defined similarly to Definition II. However, here $\chi_{s}$ is independent of joint action $a$ in $s$ but rather assumes strategy $\pi(s)$ to be played instead.

\section{Example Games}

In this chapter we study three abstract examples of stochastic games: a two-state variant of the prisoners' dilemma, a common interest game, as well as a two-state variant of matching pennies. For a definition of stochastic games see Section 2.5. 


\section{Two-State Prisoners' Dilemma}

The two-state prisoners' dilemma is a stochastic game for two players. The payoff matrices are given by:

$$
\left(A\left(s_{1}\right), B\left(s_{1}\right)\right)=\left(\begin{array}{rl}
3,3 & 0,10 \\
10,0 & 2,2
\end{array}\right),\left(A\left(s_{2}\right), B\left(s_{2}\right)\right)=\left(\begin{array}{rl}
4,4 & 0,10 \\
10,0 & 1,1
\end{array}\right) .
$$

Where $A(s)$ determines the payoff for the row player (player I) and $B(s)$ for the column player (player 2) in state $s$. The first action of each player is cooperate and the second is defect. The row player receives payoff $R^{(1)}(s, a)=A(s)_{a^{(1)}, a^{(2)}}$ while the column player receives $R^{(2)}(s, a)=B(s)_{a^{(1)}, a^{(2)}}$ for a given joint action $a=\left\langle a^{(1)}, a^{(2)}\right\rangle$ in state $s$. Similarly, the transition probabilities are given by the matrices $Q^{s \rightarrow s^{\prime}}$ where $T_{s^{\prime}}(s, a)=Q_{a^{(1)}, a^{(2)}}^{s \rightarrow s^{\prime}}$ is the probability for a transition from state $s$ to state $s^{\prime}$ when performing joint action $a$.

$$
Q^{s^{1} \rightarrow s^{2}}=\left(\begin{array}{ll}
0.1 & 0.9 \\
0.9 & 0.1
\end{array}\right), Q^{s^{2} \rightarrow s^{1}}=\left(\begin{array}{ll}
0.1 & 0.9 \\
0.9 & 0.1
\end{array}\right)
$$

The probabilities to continue in the same state after the transition are:

$$
T_{s_{1}}\left(s_{1}, a\right)=Q_{a^{(1)}, a^{(2)}}^{s_{1} \rightarrow s_{1}}=1-Q_{a^{(1)}, a^{(2)}}^{s_{1} \rightarrow s_{2}} \quad \text { and } \quad T_{s_{2}}\left(s_{2}, a\right)=Q_{a^{(1)}, a^{(2)}}^{s_{2} \rightarrow s_{2}}=1-Q_{a^{(1)}, a^{(2)}}^{s_{2} \rightarrow s_{1}}
$$

Essentially a prisoners' dilemma is played in both states, and if regarded separately defect is still a dominating strategy. One might assume that the Nash equilibrium strategy in this game is to defect at every stage. However, the only pure stationary equilibria in this game reflect strategies where one of the players defects in one state while cooperating in the other and the second player does exactly the opposite. Hence, a player betrays his opponent in one state while being exploited himself in the other state.

\section{Common Interest Game}

Another two-player, two-action and two-state game is the common interest game. Payoff and transition matrices are given below. Note that both players receive identical immediate rewards:

$$
\begin{gathered}
A\left(s_{1}\right)=B\left(s_{1}\right)=\left(\begin{array}{ll}
5 & 6 \\
6 & 7
\end{array}\right), A\left(s_{2}\right)=B\left(s_{2}\right)=\left(\begin{array}{cc}
0 & 10 \\
5 & 0
\end{array}\right), \\
Q^{s^{1} \rightarrow s^{2}}=\left(\begin{array}{ll}
0.9 & 0.9 \\
0.9 & 0.1
\end{array}\right), Q^{s^{2} \rightarrow s^{1}}=\left(\begin{array}{ll}
0.1 & 0.1 \\
0.1 & 0.9
\end{array}\right) .
\end{gathered}
$$


In this game the highest payoff is gained in state $s_{2}$ under joint action $\langle 1,2\rangle$, which is associate with a low state transition probability $Q_{1,2}^{s^{2} \rightarrow s 1}=0.1$. If however the state is switched, players are encouraged to play either joint action $\langle 1,2\rangle$ or $\langle 2,1\rangle$ in order to transition back to state $s_{2}$ with a high probability. While joint action $\langle 2,2\rangle$ maximizes immediate payoff in state $s_{1}$, the low transition probability $Q_{2,2}^{s^{1} \rightarrow s^{2}}=0.1$ hinders the return to state $s_{2}$.

\section{Two-State Matching Pennies game}

Our final example is a two-state variant of the matching pennies game. This game has a mixed Nash equilibrium with strategy profile $\pi^{(1)}=(.75, .25), \pi^{(2)}=(.5, .5)$ in state $s_{1}$ and $\pi^{(1)}=(.25, .75), \pi^{(2)}=(.5, .5)$ in state $s_{2}$. Payoff and transition matrices are given below:

$$
\begin{gathered}
\left(A\left(s_{1}\right), B\left(s_{1}\right)\right)=\left(\begin{array}{ll}
1,0 & 0,1 \\
0,1 & 1,0
\end{array}\right),\left(A\left(s_{2}\right), B\left(s_{2}\right)\right)=\left(\begin{array}{ll}
0,1 & 1,0 \\
1,0 & 0,1
\end{array}\right), \\
Q^{s^{1} \rightarrow s^{2}}=\left(\begin{array}{ll}
1 & 1 \\
0 & 0
\end{array}\right), Q^{s^{2} \rightarrow s^{1}}=\left(\begin{array}{ll}
0 & 0 \\
1 & 1
\end{array}\right) .
\end{gathered}
$$

\section{Anomalies of Piecewise Replicator Dynamics}

This section shows the shortcomings of piecewise replicators by comparing the dynamics of learning automata to predictions from the piecewise model. The layered sequence plot in Figure 4.I (page 66) and Figure 4.2 (page 67) is used to observe and describe the different dynamics. Each still image consists of three layers, the learning trace of automata $\left(L_{R-I}\right.$ with $\left.a=0.001\right)$, cell partitioning and a vector field. The learning trace in state $\mathrm{I}$ is plotted together with the cell boundaries for state 2 and vice versa. Depending on the current end-point location of the trajectory in state I we illustrate the dynamics in state 2 by plotting the vector field of the corresponding set of replicator equations. For the particular example in Figure 4.2, the trajectory in state 2 does not cross any cell boundaries and therefore the vector field in state I remains unchanged during the sequence. The learning trace in state I follows this vector field and eventually converges to joint action cooperate-defect $(C, D)$ resembling point $(1,0)$ in the reduced strategy space. During this process, the trajectory intersects multiple cell boundaries and the vector field in state 2 changes accordingly. We consult Figure 4.3 to identify cell labels for the partitioning plotted in the strategy space of state I.

The first row of Figure 4.I shows that the current end-point of the learning trajectory in state $\mathrm{I}$ is within the boundaries of cell $(C, D)$. This means, cell $(C, D)$ is active in state 2 and the dynamics are reigned by this attractor. In fact, we see that the policies 
of learning automata are attracted by this equilibrium point and approximately follow the vector field toward $(1,0)$.

Let us now consider the third row of Figure 4.I and the second row of Figure 4.2. Here, the current end-points of the trajectories in state I correspond to the cells $(D, C)$ and $(C, D)$ respectively. In the former case, the vector field plot shows arrows near the end of the trace that point toward $(0,1)$. However, the automata policies continue on an elliptic curve and therefore approximately progress into the direction of $(1,1)$ rather than $(0,1)$. The latter case shows even greater discrepancies between vector field and policy trajectory. The vector field predicts movement toward $(0,0)$, while the trajectory trace continues convergence to $(0,1)$. We now attempt to given an explanation for these artifacts by performing a sensitivity analysis of vector field plots.

Figure 4.3 (page 68) displays the strategy space partitioning for state 2 depending on strategies in state I (left) as well as a magnification of the subspace near the origin (right). We specifically focus on this subspace and compute the average reward games for three strategy profiles, i.e., $(0.015,0.015),(0.06,0.01)$ and $(0.01,0.06)$. Each strategy profile corresponds to one of three cells, $(C, C),(C, D)$ and $(D, C)$ respectively, as indicated in the clipped section to the right in Figure 4.3. The computed average reward game payoff matrices are used to derive the replicator dynamics and visualize the vector field plots. Figure 4.4 compares the field plots of the three specific average reward games with the dynamics for the corresponding cells obtained by sampling. On the highest level, i.e., presence and convergence to strong attractors, all pairs match. This is clear, since average reward games for specific points within a cell sustain the same equilibrium combination. However, in order to examine the anomaly in Figure $\mathbf{4 . 2}$ (second row), we consider especially the direction of field plots in the area around the trajectory end-point, which in this case is circa $(0.15,0.4)$. In this area dynamics for cells $(C, D)$ and $(D, C)$ show clear qualitative differences from the field plots for corresponding specific strategy profiles $(0.06,0.01)$ and $(0.01,0.06)$. Furthermore, the field plots to the left in Figure 4.4 are consistent with the learning trajectory sequence displayed in Figure 4.2.

The vector field plots of piecewise replicator dynamics predict the learning dynamics only on the highest level (see Figure 4.4, page 69). However, due to the coupling of states, qualitative anomalies occur. Furthermore, piecewise replicators show discontinuities due to discrete switching of dynamics each time a cell boundary is crossed. These discontinuities are neither intuitive nor reflected in real learning behavior. Additionally, these artifacts potentially yield profound impact if the number of states is increased.

The next section aims to alleviate the shortcomings of piecewise replicators by proposing a new approach to model the learning dynamics in stochastic games more accurately. 

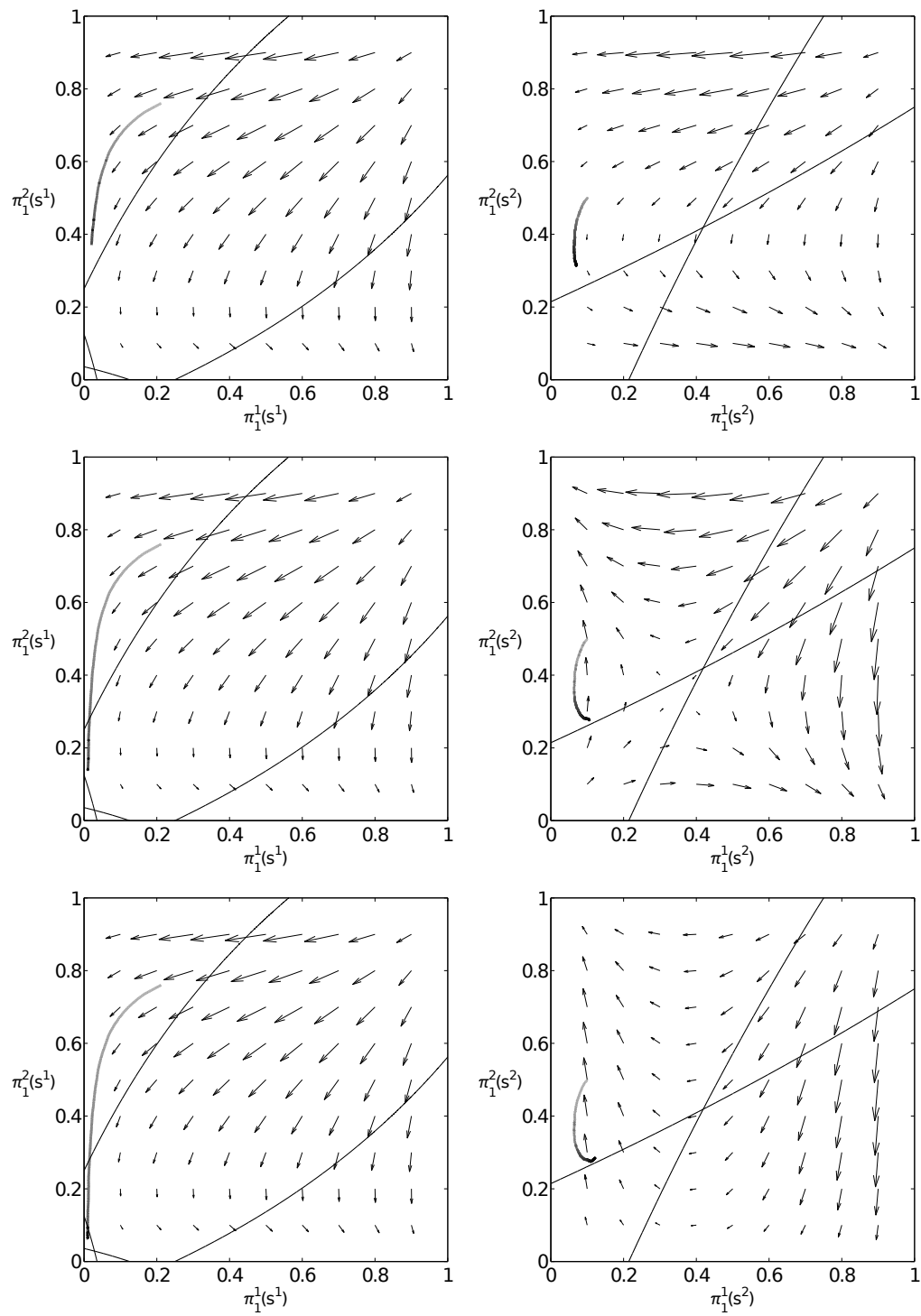

Figure 4.I: Trajectory plot for learning automata in the two-state prisoners' dilemma. Each boundary intersection in state I (left column) causes a qualitative change of dynamics in state 2 (right column). 

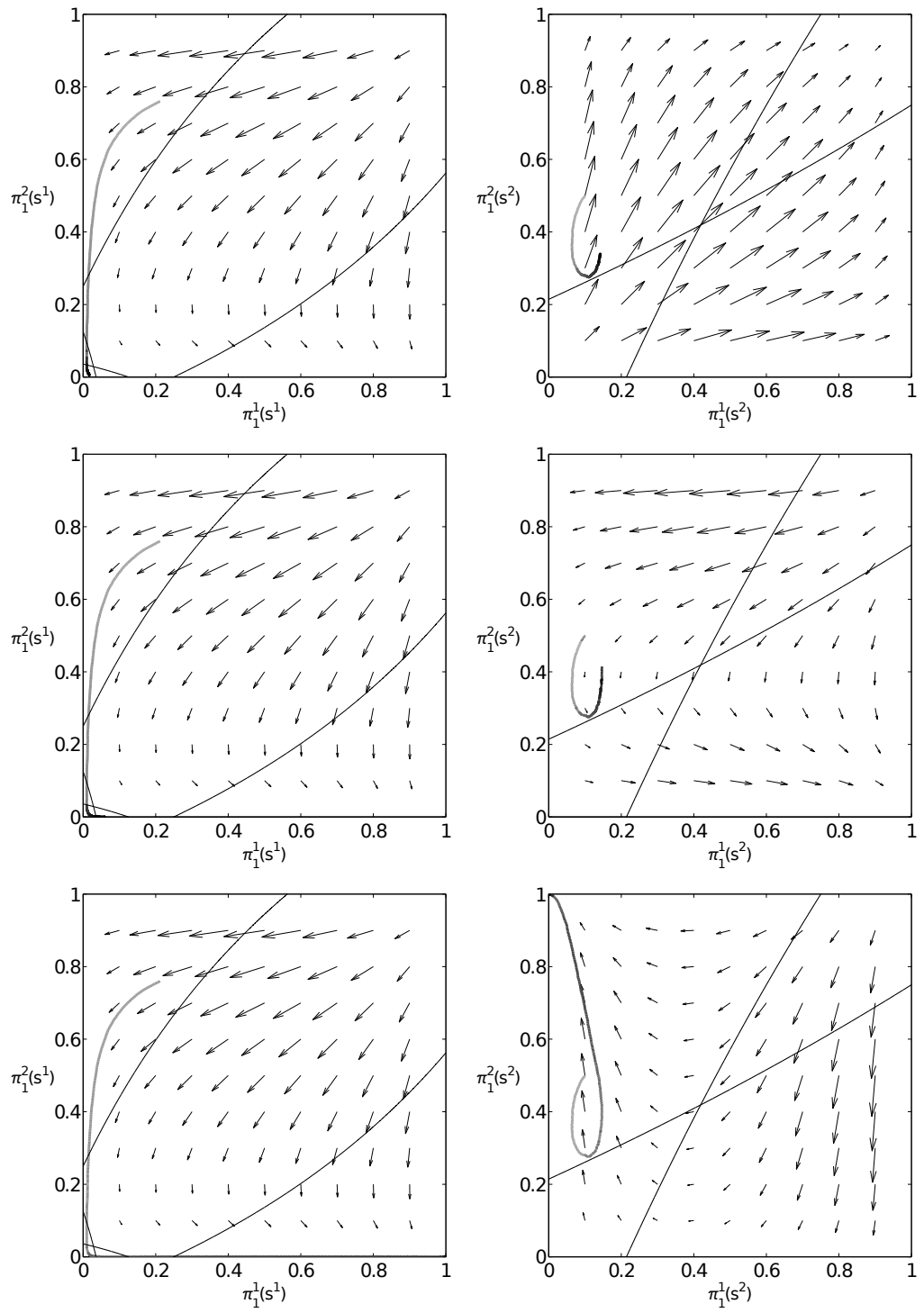

Figure 4.2: Continuation of trajectory sequence in Figure 4.I. The second row shows a clear anomaly: the learning trajectory does not approximate the dynamics given by the corresponding cell replicator equations. 

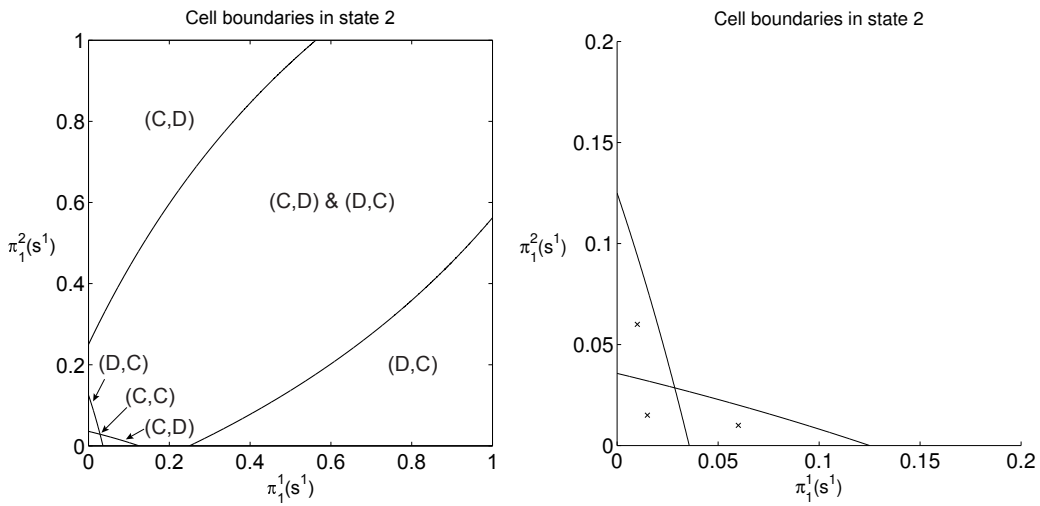

Figure 4.3: Cell partitioning and location of specific strategy profiles, i.e., $(0.015,0.015)$, $(0.06,0.01)$ and $(0.01,0.06)$, for vector field sensitivity analysis in the two-state prisoners' dilemma.

\subsection{State-Coupled Replicators Dynamics}

Piecewise replicators are an implementation of the paradigm of piecewise dynamical systems and therefore inherently limited to a qualitative approximation of the underlying, intrinsic behavior. Anomalies occur if this approximation is deficient for some subspace of the strategy space. This observation directly suggest either

a) to refine the cell partitioning, or

b) to strive for direct state coupling, discarding the piecewise model.

Refining the cell partitioning is not straightforward. One might consider to separate the disjointed parts of the strategy subspace, previously covered by a single cell. More precisely, this would lead to two separate cells each for pure equilibria $(C, D)$ and $(D, C)$ in state 2 of the two-state prisoners' dilemma. However, this approach is not in line with the argument that a cell should reflect the subspace in which a certain attractor reigns.

The second option is most promising since it eliminates undesired discontinuities induced by discrete cell switching and furthermore avoids approximation anomalies. Accordingly, in the next section we derive a new model for state-coupled learning dynamics in stochastic games. We call this approach state-coupled replicator dynamics.

\section{Definition}

We reconsider the replicator equations for population $x$ as given in (3.2):

$$
\frac{\partial x_{i}}{\partial t}=x_{i}\left[(A y)_{i}-x^{\top} A y\right]
$$



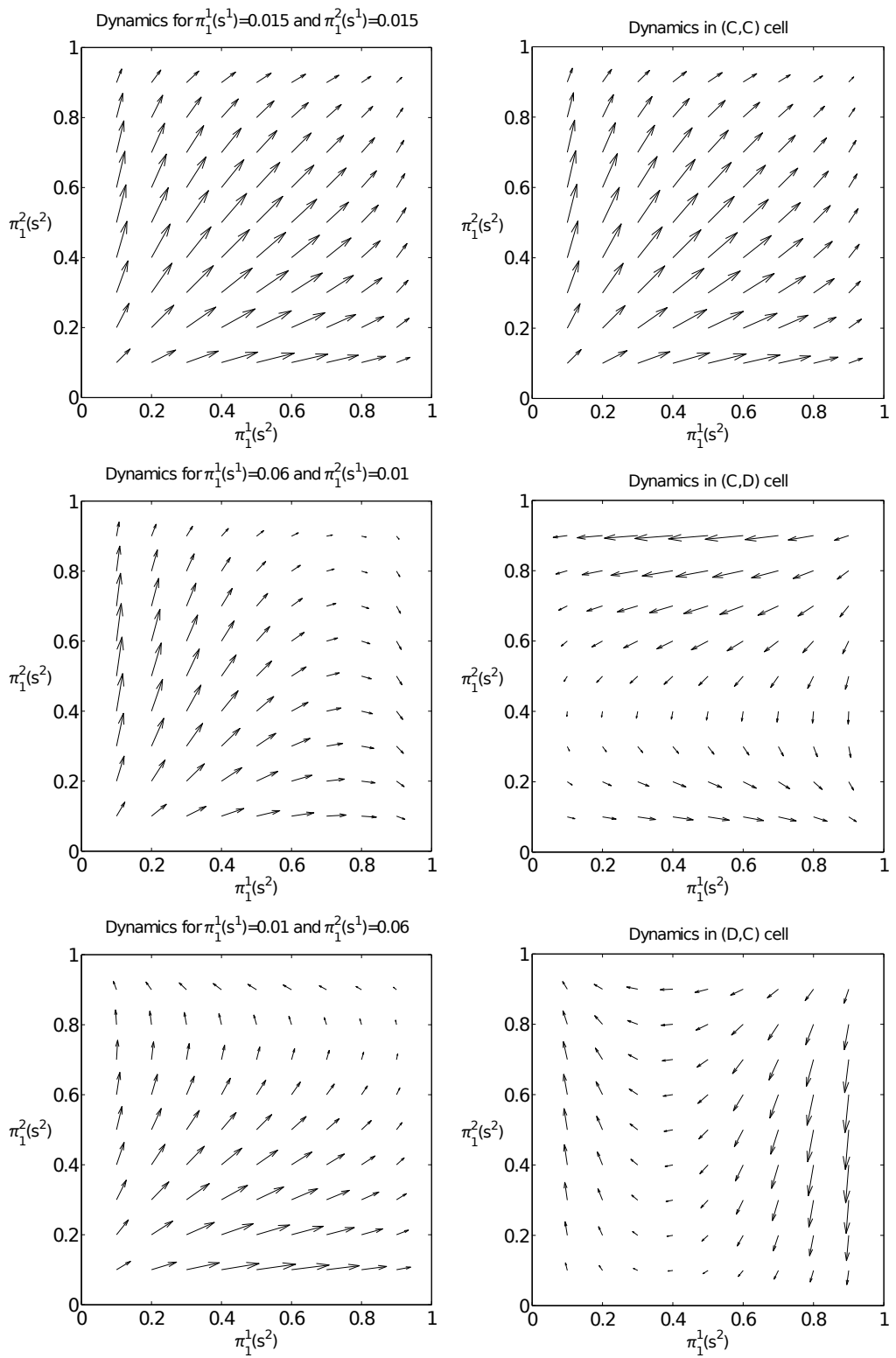

Figure 4.4: Vector field sensitivity analysis for piecewise replicator dynamics. Cell replicator equations are compared to the dynamics of specific average reward games for the following strategy profiles: $(0.015,0.015),(0.06,0.01)$ and $(0.01,0.06)$. 
Essentially, the payoff of an individual in population $x$, playing pure strategy $i$ against population $y$, is compared to the average payoff of population $x$. In the context of an average reward game $\bar{G}$ with payoff function $\bar{R}$ the expected payoff for player $i$ and pure action $j$ is given by:

$$
f_{j}^{(i)}(s)=\sum_{a \in \prod_{l \neq i} A^{(l)}(s)}\left(\bar{R}^{(i)}\left(s, a^{\prime}\right) \prod_{l \neq i} x_{a^{(l)}}^{(l)}(s)\right),
$$

where $a^{\prime}=\left(a^{(1)}, \ldots, a^{(i-1)}, j, a^{(i)}, \ldots, a^{(n)}\right)$. This means that we enumerate all possible joint actions $a$ with fixed action $j$ for agent $i$. In general, for some mixed strategy $p$, agent $i$ receives an expected payoff of:

$$
f^{(i)}(s, p)=\sum_{j \in A^{(i)}(s)}\left[p_{j} \sum_{a \in \prod_{l \neq i} A^{(l)}(s)}\left(\bar{R}^{(i)}\left(s, a^{\prime}\right) \prod_{l \neq i} x_{a^{(l)}}^{(l)}(s)\right)\right] .
$$

If each player $i$ is represented by a population $x^{(i)}$, we can set up a system of differential equations, each similar to (4.3), where the payoff matrix $A$ is substituted by the average reward game payoff $\bar{R}$. Furthermore, $y$ now represents all remaining populations $x^{(l)}$ where $l \neq i$.

Definition 15. The multi-population state-coupled replicator dynamics are defined by the following system of differential equations:

$$
\frac{\partial x_{j}^{(i)}(s)}{\partial t}=x_{j}^{(i)} \chi_{s}(X)\left[f^{(i)}\left(s, e_{j}\right)-f^{(i)}\left(s, x^{(i)}(s)\right)\right],
$$

where $e_{j}$ is the $j^{\text {th }}$-unit vector. $f^{(i)}(s, p)$ is the expected payoff for an individual of population i playing some strategy $p$ in state s. Fitness $f^{(i)}$ is defined as

$$
f^{(i)}(s, p)=\sum_{j \in A^{(i)}(s)}\left[p_{j} \sum_{a \in \prod_{l \neq i} A^{(l)}(s)}\left(\bar{R}^{(i)}\left(s, a^{\prime}\right) \prod_{l \neq i} x_{a^{(l)}}^{(l)}(s)\right)\right],
$$

where $\bar{R}$ is the payoff function of $\bar{G}(s, X)$ with $X=\left\langle x^{(1)}, \ldots, x^{(n)}\right\rangle$ and

$$
a^{\prime}=\left(a^{(1)}, \ldots, a^{(i-1)}, j, a^{(i)}, \ldots, a^{(n)}\right) .
$$

Furthermore, $\chi(X)$ is the stationary distribution over all states $S$ under strategy profile $X$, with

$$
\sum_{s \in S} \chi_{s}(X)=1
$$


and

$$
\chi_{s}(X)=\sum_{z \in S}\left[x_{z}(X) \sum_{a \in \prod_{i=1}^{n} A^{(i)}(s)}\left(T_{s}(z, a) \prod_{i=1}^{n} x_{a_{(i)}}^{(i)}(s)\right)\right] .
$$

In total this system has $m=\sum_{s \in S} \sum_{i=1}^{n}\left|A^{(i)}(s)\right|$ replicator equations.

The piecewise replicator dynamics rely on a cell partitioning, where the dynamics in each cell are approximated by a static set of replicator equations. In contrast, the state-coupled replicator dynamics use direct state-coupling by incorporating the expected payoff in all states under current strategies, weighted by the frequency of state occurrences.

\section{Results and Discussion}

This section sets the newly proposed state-coupled replicator dynamics in perspective by comparing their dynamics with learning automata and piecewise replicators.

Figure 4.5 (page 72) plots a single trace each for learning automata as well as piecewise and state-coupled replicator dynamics in the two-state prisoners' dilemma. All three trajectories converge to the same equilibrium point. The piecewise replicator dynamics clearly show discontinuities due to switching dynamics, triggered at each cell boundary intersection. Furthermore, the trace in state 2 enters the subspace for cell $(D, D)$ in state I, while both trajectories for learning automata and state-coupled replicators remain in cell $(C, D)$. This comparison clearly shows that state-coupled replicators model the learning dynamics more precisely.

In Figure 4.6 (page 73) we compare multiple trajectory traces originating from one fixed strategy profile in state I and a set of randomly chosen strategies in state 2 . This allows to judge the predictive quality of piecewise- and state-coupled replicator dynamics with respect to the learning curves of automata games.

Finally, Figure 4.7 (page 74) presents trajectory plots for the common interest game. Automata games and learning dynamics modeled by state-coupled replicator dynamics are compared. The strong alignment between model and real learning traces are evident in this game just as for the two-state prisoners' dilemma.

The new proposed state-coupled replicator dynamics directly describe the coupling between states and hence no longer rely on an additional layer of abstraction like piecewise cell dynamics. We observe in a variety of results that state-coupled replicator dynamics model multi-agent reinforcement learning in stochastic games by far better than piecewise replicators. 

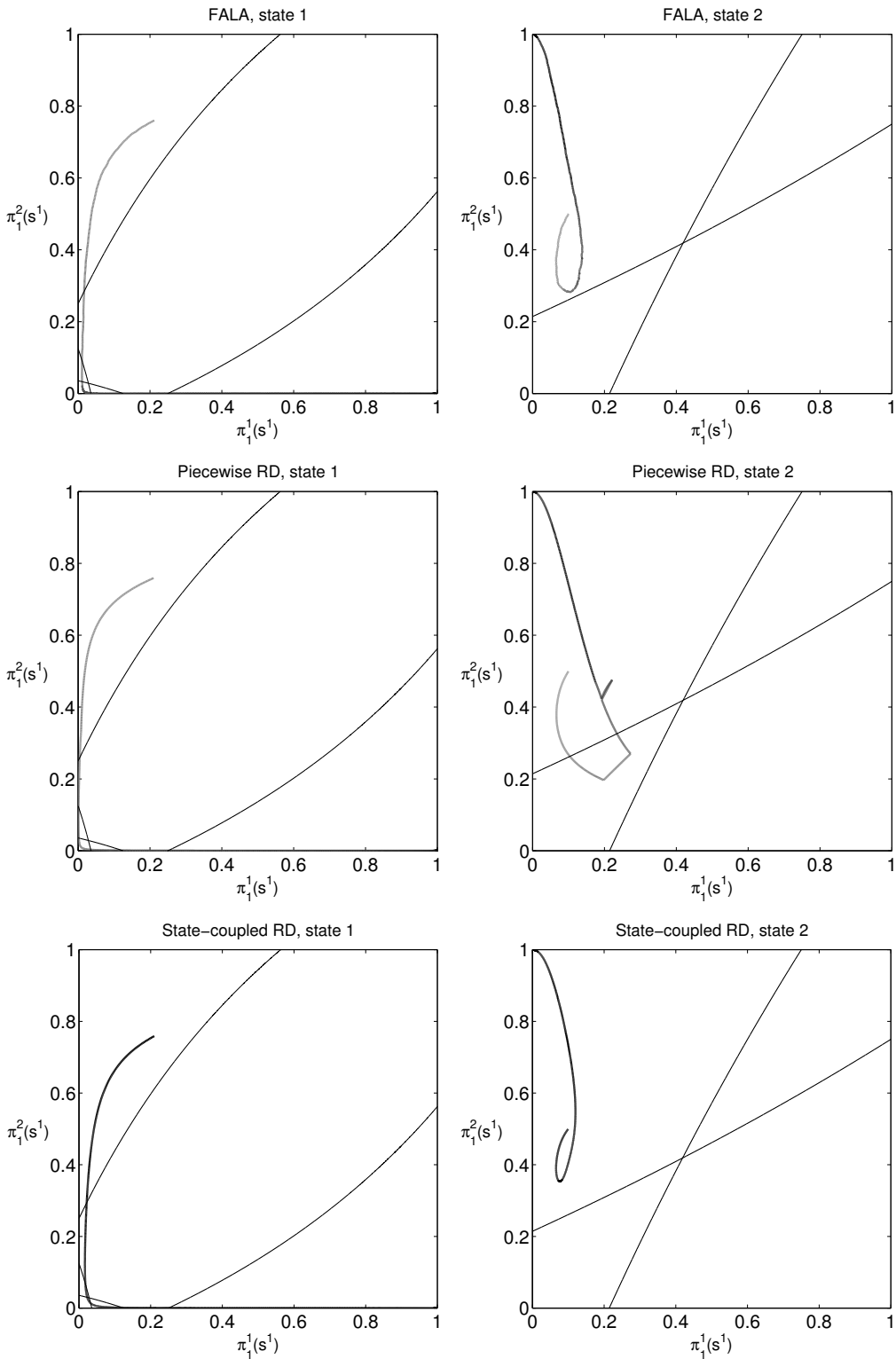

Figure 4.5: Comparison between a single trajectory trace of learning automata, piecewise and state-coupled replicator dynamics in the two-state prisoners' dilemma. The piecewise replicator dynamics clearly show discontinuities, while the state-coupled replicators model the learning dynamics more accurately. 

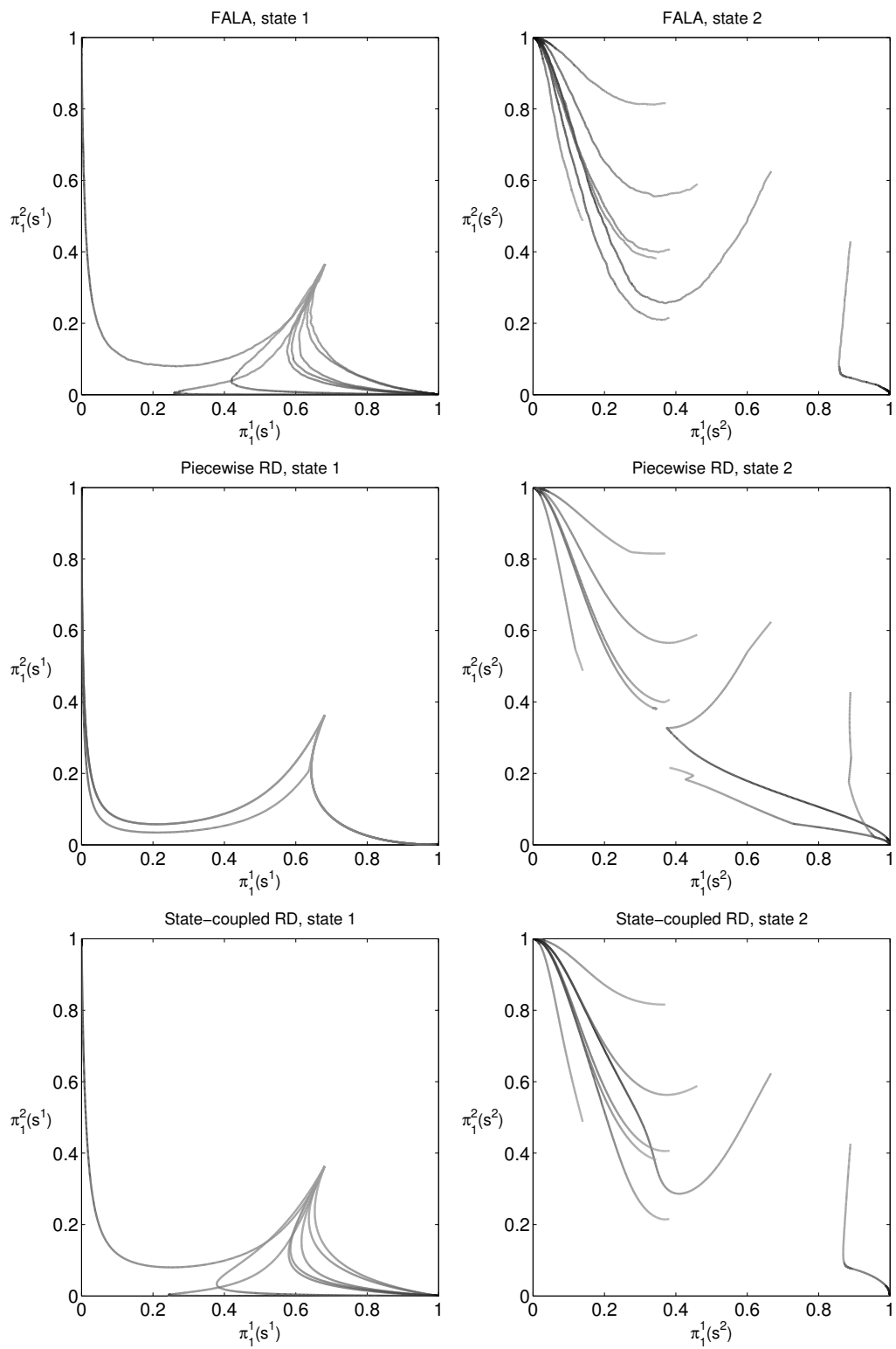

Figure 4.6: Comparison between trajectory traces of learning automata, piecewise and state-coupled replicator dynamics in the two-state prisoners' dilemma. Initial action probabilities are fixed in state I while a set of 8 random strategy profiles is used in state 2. Wrong convergence and discontinuities are observed for piecewise replicators. 

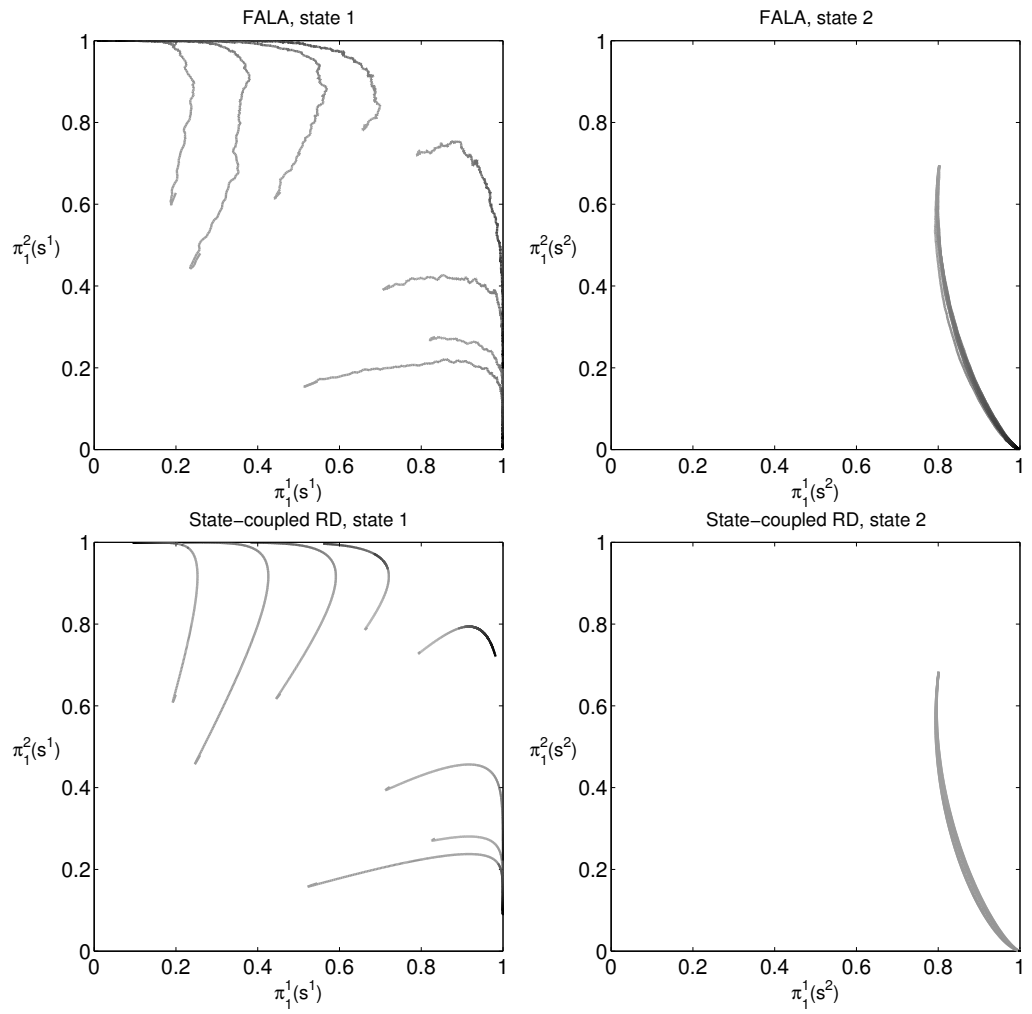

Figure 4.7: Comparison between trajectory traces of learning automata and statecoupled replicator dynamics in the common interest game. Initial action probabilities are fixed in state 2 while a set of 8 random strategy profiles is used in state $\mathrm{I}$.

\subsection{Symmetric State-Coupled Replicator Dynamics}

In the previous section we have derived the general continuous-time multi-population state-coupled replicator equations. Similar to the stateless case, for a symmetric twoplayer stochastic game we can define population dynamics that choose two individuals at random from the same population in each state.

Assume we have a symmetric two-player stochastic game with $k$ states $S=\left\{s_{1}, \ldots, s_{k}\right\}$ and corresponding payoff matrices $A(s)$ for $s \in S$. The game is symmetric if and only if $A(s)=A(s)^{\top}$ for all states $s \in S$. The full system state consists of $k$ populations, one in each state:

$$
X=\left\langle x\left(s_{1}\right), \ldots, x\left(s_{k}\right)\right\rangle .
$$

The average payoff in state $s$ given $X$ is:

$$
\bar{f}(s, X)=x(s)^{\top} A(s) x(s)
$$




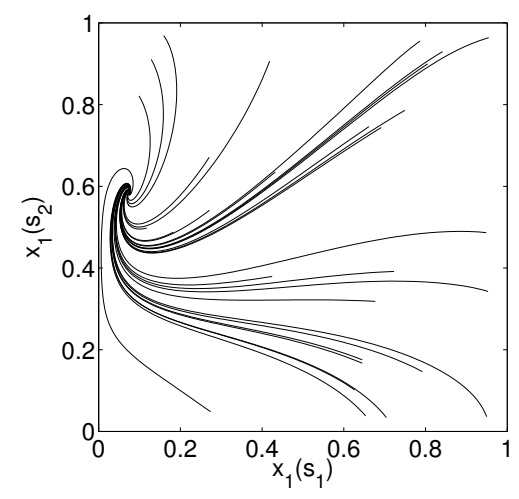

Figure 4.8: Symmetric replicator dynamics in the two-state prisoners' dilemma. The graph plots trajectories of cooperation in state $s_{1}$ against cooperation in state $s_{2}$.

The fitness of a population $p$ in state $s$ and $x\left(s^{\prime}\right)$ in all other states $x^{\prime} \in S \backslash\{s\}$ is computed according to the average reward game payoff formulation:

$$
f(s, p, X)=\chi_{s} p^{\top} A(s) x(s)+\sum_{s^{\prime} \in S\{s\}} \chi_{s^{\prime}} \bar{f}\left(s^{\prime}, X\right),
$$

with fixed populations in all state $s^{\prime} \neq s$ and population $p$ in state $s$.

What is the stationary distribution $\chi$ for a symmetric stochastic game? Suppose the game is in state $s$, two individuals are drawn at random from the population $x(s)$ and play according to their phenotypes. The probability of drawing an individual of type $i$ and an individual of type $j$ from the population is $x(s)_{i} x(s)_{j}$. Therefore, we can compute the transition probability from state $s$ to $s^{\prime}$ as follows:

$$
p_{s, s^{\prime}}=\sum_{i, j} T_{s^{\prime}}(s,\langle i, j\rangle) x_{i}^{(s)} x_{j}^{(s)}
$$

where $T_{s^{\prime}}(s,\langle i, j\rangle)$ is the probability of transitioning from state $s$ to $s^{\prime}$ given joint action $\langle i, j\rangle$.

The symmetric state-coupled replicator dynamics for an irreducible stochastic game with stationary distribution $\pi$ and fitness function $f$ are defined as:

$$
\frac{\partial x(s)_{i}}{\partial t}=x(s)_{i} \chi_{s}\left[f\left(s, e_{i}, X\right)-f(s, x(s), X)\right] .
$$

Figure 4.8 shows the symmetric replicator dynamics in the 2 state prisoners' dilemma. Trajectories show the evolution of $\left\langle x_{1}\left(s_{1}\right), x_{1}\left(s_{2}\right)\right\rangle$, i.e., cooperation in state $s_{1}$ against cooperation in state $s_{2}$. There is one interior fixed point at $(0.074,0.582)$ with $\chi_{s_{1}}=$ 0.7 and $\chi_{s_{2}}=0.3$. 


\subsection{State-Coupled Replicator Dynamics with Mutation}

So far we have focused on deriving predictive models for the learning dynamics of existing multi-agent reinforcement learners. These models help to gain deeper insight and allow to tune parameter settings. In this section we demonstrate the inverse approach, designing a dynamical system that converges to pure and mixed Nash equilibria in stochastic games and "reverse engineering" that system, resulting in a new multi-agent reinforcement learning algorithm, called RESQ-learning.

Results for stateless games provide evidence that exploration is the key to prevent cycling around attractors Tuyls et al. (2003b). Hence, we aim to combine the exploration-mutation term of FAQ-learning dynamics with state-coupled replicator dynamics.

\section{Linking Learning Automata and Q-learning Dynamics}

First, we link the dynamics of learning automata and $Q$-learning for the stateless case. We recall from Section 3.3 that the learning dynamics of LA correspond to the standard multi-population replicators scaled by the learning rate $\alpha$ :

$$
\frac{\partial x_{i}}{\partial t}=\alpha x_{i}\left[(A y)_{i}-x^{\top} A y\right]
$$

The FAQ-learning replicator dynamics contain a selection part equivalent to the multipopulation replicator dynamics, and an additional mutation part originating from the Bolzmann exploration scheme:

$$
\begin{aligned}
\frac{\partial x_{i}}{\partial t} & =\beta x_{i}\left(\tau^{-1}\left[(A y)_{i}-x^{\top} A y\right]-\ln x_{i}+\sum_{j} x_{j} \ln x_{j}\right) \\
& =\beta x_{i} \tau^{-1}\left[(A y)_{i}-x^{\top} A y\right]-\beta x_{i}\left(\ln x_{i}+\sum_{j} x_{j} \ln x_{j}\right)
\end{aligned}
$$

The learning rate of FAQ-learning is now denoted by $\beta$. Let us assume $\alpha=\beta \tau^{-1} \Rightarrow$ $\beta=\alpha \tau$. Note that from $\beta \in[0,1]$ follows: $0 \leq \alpha \tau^{-1} \leq 1$. Then we can rewrite the FAQ-learning replicator equations (4.5) as follows:

$$
\frac{\partial x_{i}}{\partial t}=\alpha x_{i}\left[(A y)_{i}-x^{\top} A y\right]-\alpha \tau x_{i}\left(\ln x_{i}+\sum_{j} x_{j} \ln x_{j}\right) .
$$

Note that, in the limit $\tau \rightarrow 0$ the mutation term collapses and the dynamics reduce again to (4.4). 


\section{State-Coupled Replicator Dynamics with Mutation}

Now that we have established the connection between the learning dynamics of FAQlearning and learning automata, extending this link to multi-state games is straightforward. The mutation term $-\tau\left(\ln x_{i}+\sum_{j} x_{j} \ln x_{j}\right)$ in (4.6) is solely dependent on the population $x$ and thus independent of any payoff computation. Therefore, the average reward game remains the sound measure for the limit of the average of stage rewards under the assumptions made in Section 4.I. The equations of the dynamical system in (4.2) are complemented with the mutation term, resulting in the following continuous time state-coupled replicator equations with mutation:

$$
\begin{aligned}
\frac{\partial x_{j}^{(i)}(s)}{\partial t}=x_{j}^{(i)} \chi_{s}(X)[ & {\left[f^{(i)}\left(s, e_{j}\right)-f^{(i)}\left(s, x^{(i)}(s)\right)\right] } \\
& \left.-\tau\left(\ln x_{j}^{(i)}(s)+\sum_{k} x_{k}^{(i)}(s) \ln x_{k}^{(i)}(s)\right)\right]
\end{aligned}
$$

In the next section we introduce the corresponding RESQ-learning algorithm.

\section{RESQ-Learning}

In Section 4.I we have shown that maximizing the expected average stage reward over interim immediate rewards relates to the average reward game played in state-coupled replicator dynamics. We reverse this result to obtain a learner equivalent to statecoupled replicator dynamics with mutation.

Analogous to the description in Section 2.4, a network of learners is used for each agent. The reward feedback signal is equal to (2.22) while the update rule now incorporates the same exploration term as in (4.7). If $a=a_{t}$ :

$$
\pi_{t+1}(a)=\pi_{t}(a)+\alpha\left[R_{t}\left(1-\pi_{t}(a)\right)-\tau\left(\ln \pi_{t}(a)+\sum_{k} \pi_{t}(k) \ln \pi_{t}(k)\right)\right],
$$

otherwise:

$$
\pi_{t+1}(a)=\pi_{t}(a)+\alpha\left[-R_{t} \pi_{t}(a)-\tau\left(\ln \pi_{t}(a)+\sum_{k} \pi_{t}(k) \ln \pi_{t}(k)\right)\right] .
$$

Hence, RESQ-learning is essentially a multi-state policy iterator using exploration equivalent to the Boltzmann policy generation scheme. 


\section{Results and Discussion}

This section sets the newly proposed RESQ-learning algorithm in perspective by examining the underlying dynamics of state-coupled replicator dynamics with mutation and traces of the resulting learning algorithm.

First, we explore the behavior of the dynamical system, as derived in (4.7), and verify the desired convergence behavior, i.e., convergence to pure and mixed Nash equilibria. Figure 4.9 shows multiple trajectory traces in the two-state prisoners' dilemma, originating from random strategy profiles in both states. Analysis reveals that all trajectories converge close to either one of the two pure Nash equilibrium points described in Section 4.I. As mentioned before for the stateless case, constant temperature prohibits full convergence. Figure 4.Io shows trajectory traces in the two-state matching pennies game. Again, all traces converge close to Nash, thus affirming the statement that exploration-mutation is crucial to prevent cycling and to converge in games with mixed optimal strategies.

Figure 4.II (page 80) shows a comparison between state-coupled replicator dynamics (SC-RD), the RESQ-dynamics as in (4.7), and an empirical learning trace of RESQlearners. As mentioned above, "pure" state-coupled replicator dynamics without the exploration-mutation term fail to converge. The trajectory of this dynamical system exhibits cycling behavior around the mixed Nash equilibrium. RESQ-dynamics successfully converge $\epsilon$-near to the Nash-optimal joint policy. Furthermore, we present the learning trace of two RESQ-learners in order to judge the predictive quality of the corresponding state-coupled dynamics with mutation. Due to the stochasticity involved in the action selection process, the learning trace is more noisy. However, we clearly observe that RESQ-learning indeed successfully inherits the convergence behavior of state-coupled replicator dynamics with mutation. 

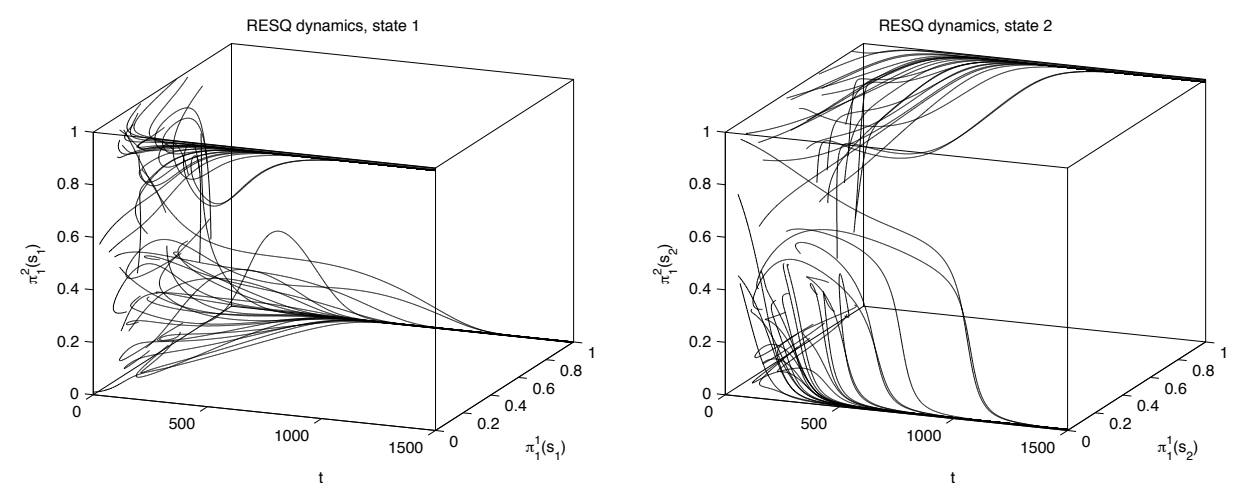

Figure 4.9: RESQ-learning dynamics $(\alpha=0.004, \tau=0.02)$ in the two-state prisoners' dilemma.
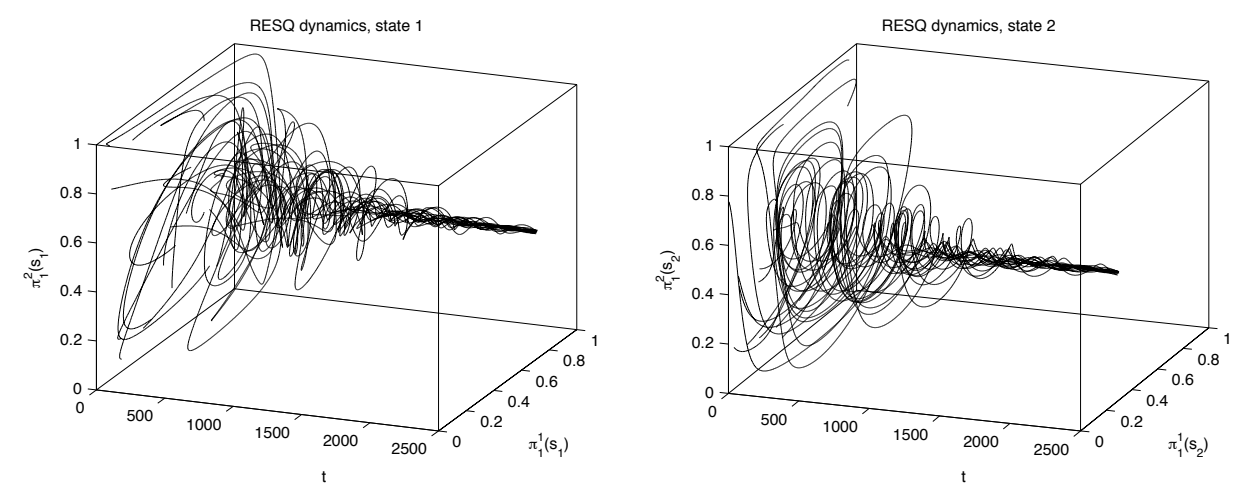

Figure 4.Io: RESQ-learning ( $\alpha=0.004, \tau=0.04$ ) in the two-state matching pennies game. 

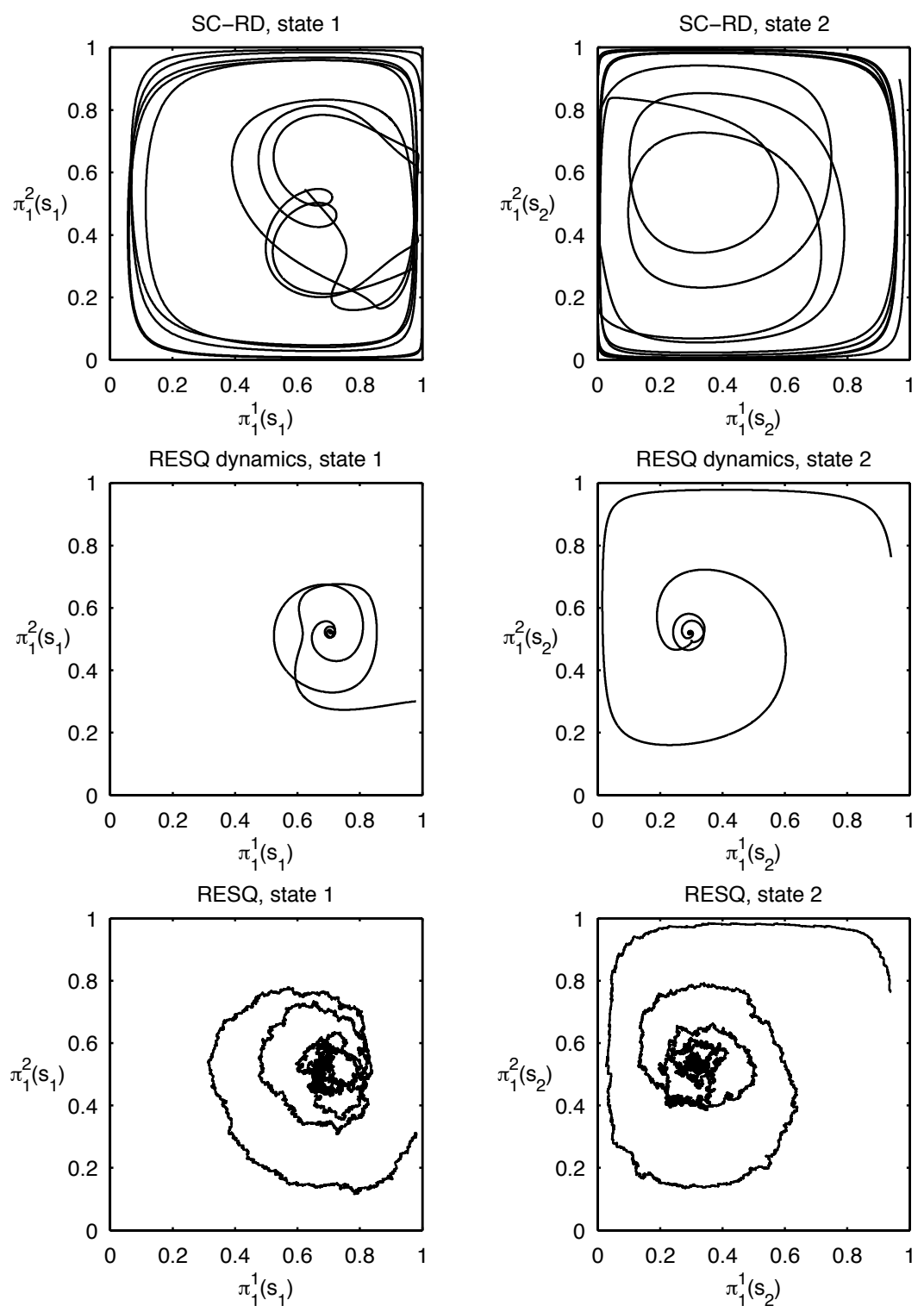

Figure 4.II: Comparison between SC-RD dynamics, RESQ dynamics and RESQ-learning $(\alpha=0.004, \tau=0.04)$ in the two-state matching pennies game. 


\subsection{Summary}

This chapter has extended the link between evolutionary game theory and multi-agent reinforcement learning to stochastic games. We have formalized and generalized the piecewise replicator dynamics, resulting in a list of definitions that includes average reward games, pure equilibrium cells, and piecewise replicator dynamics. We identified shortcomings of piecewise replicator dynamics, i.e., discontinuities and occurrences of qualitative anomalies. State-coupled replicator dynamics were proposed to alleviate these disadvantages. The derived findings and the preceding formalization of piecewise replicator dynamics are incorporated in the design of this new model of learning dynamics in stochastic games. In particular, the continuous time population dynamics for both symmetric and general stochastic games are defined. In addition, we present a comparative study of networks of learning automata to piecewise and state-coupled replicator dynamics. Results show that state-coupled replicators model learning dynamics in multi-state games more accurately than their predecessor, the piecewise approach. Furthermore, RESQ-learning has been introduced by reverse engineering state-coupled replicator dynamics injected with the Q-learning Boltzmann mutation scheme. We have provided empirical confirmation that RESQ-learning successfully inherits the convergence behavior of its evolutionary counterpart. Results show that RESQ-learning provides convergence to pure as well as mixed Nash equilibria in a selection of stochastic multi-agent games. 

Part II

Applications 



\section{5 \\ Take-it or Leave-it Negotiation}

The focus of this chapter is on the analysis of computational strategies for task settings where there is an interaction among goals, tasks required to achieve these goals, and resources needed for completing tasks. Despite their prevalence in the real world, the analysis of players' strategies in such task settings is challenging due to the large strategy space and bounded rationality that characterizes human behavior. We provide a method to reduce complex bargaining settings to a set of smaller games that make basic assumptions about the strategies employed by players in the games in a way that significantly facilitates this analysis. These heuristics, called "metastrategies" have been shown to reduce the analysis of large-scale interactions such as auctions or poker (Walsh et al., 2002; Phelps et al., 2007; Ponsen et al., 2009).

Our empirical study is based on a commonly used and freely available test-bed for modeling task settings called the Colored Trails (CT) framework (Gal et al., 20Io). CT provides an analogue to the ways in which goals, tasks and resources interact in realworld settings, but abstracts away the complexities of real-world domains. CT includes a family of games that are parameterized to allow for increasing complexity along a number of dimensions, such as the number of players, the availability of and access to information, the dependency relationships that hold between players, and the communication protocol. It supports comparisons of the performance of different computational strategies for interacting in groups comprising people and computer agents as well as solely computer agents. The framework has been used extensively in settings as diverse as repeated negotiation, interruption management, team formation and behavior analysis in space-flight simulations (Gal and Pfeffer, 2007; Kamar et al., 2009; Hennes et al., 2009a). ${ }^{\mathrm{I}}$

This chapter extends the notion of metastrategies to players' bargaining behavior in general CT settings. Specifically, it provides a mapping between a particular CT task setting and normal-form games in a way that preserves much of the strategic qualities of the original setting. We define metastrategies for each player that are domain-

\footnotetext{
${ }^{\text {I }}$ Colored Trails is free software and is available for download at: http://www.eecs.harvard.edu/ai/ct
} 
independent and make minimal assumptions about the way other players make decisions. These metastrategies allow a reduction to canonical bilateral social dilemma games such as prisoners' dilemma, stag hunt, and ultimatum games. The mapping from CT game instances to well-known social dilemmas allows to compare participants' behavior in CT with prior results from these smaller, more traditional settings.

We demonstrate this approach in a three-player CT configuration in which two proposer players make take-it-or-leave-it offers to a responder player. We define several metastrategies for proposers in this game that make varying assumptions about how responders make decisions. We lay down a set of criteria that make generated CT game instances worthwhile (or "strategically interesting") for human players. Results from simulation experiments that sample thousands of such strategically interesting CT game instances confirm that players' outcomes from playing metastrategies in the original game instances correspond to the outcomes from playing the same strategies in the reduced canonical form games. We also show how to extend this approach to other CT variants that include a varying number of proposers and responders. An evolutionary game-theoretic analysis supports this extension.

\section{Related Work}

Our work relates to past studies spanning the empirical and behavioral game theoretic literature. Work in the empirical game theoretic literature has facilitatated equilibria computation by abstracting a large strategy space to a small number of metastrategies (Walsh et al., 2002, 2004). The methodology allows to compute expected payoffs to players as a function of the joint strategy space, identify equilibria, and analyze the evolutionary dynamics. This approach has been implemented in several domains in which expert knowledge is available to assist in the establishment of suitable heuristics. Ponsen et al. (2009) have used heuristics in the game of Poker to represent players' aggression-factors (Rock, Shark, Gambler and Fish) and to facilitate learning during their play (Ponsen et al., 2008). In auctions, heuristic payoff tables have been used to represent common trading strategies such as generating random bids subject to minimal constraints as well as adaptive strategies that consider the history of past trades or have access to varying levels of information (Gjerstad and Dickhaut, I998; Phelps et al., 2006, 2007; Kaisers et al., 2009; Hennes et al., 2012a). Our work extends these studies in defining metastrategies in multiplayer task settings that do not depend on expert knowledge, and in demonstrating this approach using empirical simulation.

Our work also relates to a series of canonical studies in behavioral economics on ultimatum games (Güth et al., I982; Fehr and Schmidt, I999; Henrich et al., 2004; Oosterbeek et al., 2004). Surprisingly little work exists on extending the ultimatum game beyond two players. Grosskopf (2003) has studied ultimatum game settings with three responders and one proposer. If all three responders reject the offer, all players receive 
nothing. Otherwise a random draw determines, which of the accepting responders is matched with the proposer. Because responders compete for a single proposal, the acceptable share for the proposer is significantly higher than in the traditional game setting. Another example is an n-player ultimatum game (de Jong et al., 2008) in with each player takes a share of the reward one by one until the last player has the deciding power to accept what ever is left or to reject - thus leaving everybody with nothing. A multiplayer ultimatum graph game is studied by Chang et al. (2010). Players are connected by an interaction graph and each round choose a partner to play a bilateral ultimatum game. In particular, the stability conditions for the survival of a "fair" society of agents are investigated.

\section{I Colored Trails}

Our study is based on the Colored Trails (CT) framework, a family of games that are played by two or more participants on a board of colored squares. The basic CT board includes players' icons and goal squares, but configurations may also include traps or other features needed to model a task situation. We begin our study with a threeplayer CT configuration that includes a board of 4x4 squares, colored in one of five colors. Each player possesses a piece located on the board and a set of colored chips. A colored chip can be used to move a player's piece to an adjacent square (diagonal movement is not allowed) of the same color. The general goal is to position pieces onto or as close as possible to a goal location indicated by a flag. Each player receives points purely based on its own performance. There are distinct roles in the game: two proposer(s) (termed $P 1$ and $P 2$ ) and a single responder (termed $R$ ). Figures 5.I(a) and 5.I(c) show two examples of game instances that include game boards showing the goal and player locations, as well as the chip sets that have been allocated to each player. The CT game is divided into the following sequence of four phases.

a) Initial phase. The game board and the chip sets are allocated to the players. This initial phase allows participants to locate their own piece on the board and reason about the game. For example, in Figure 5.I(a), proposer $P 1$ is missing a green chip to get to the goal (by moving left-up-up), proposer $P 2$ is missing a gray or green chip (moving up-up-right or up-right-up) to get to the goal, and responder $R$ is missing a gray chip and a blue chip to get to the goal (moving right-3up-right). The game state is fully observable at this point, except that proposers cannot see each other's chips.

b) Proposal phase. The two proposers can make chip exchange offers to the responder. Both proposers make offers to the responder simultaneously; they cannot observe each other's offer. 


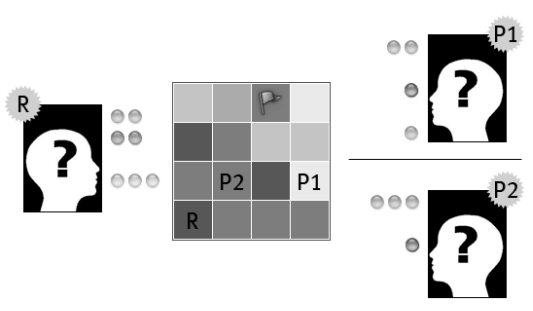

(a) First Colored Trails example instance.

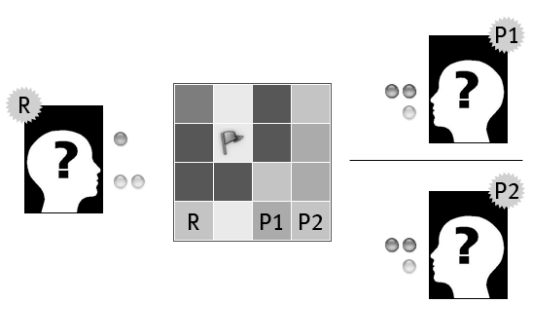

(c) Second Colored Trails example instance.
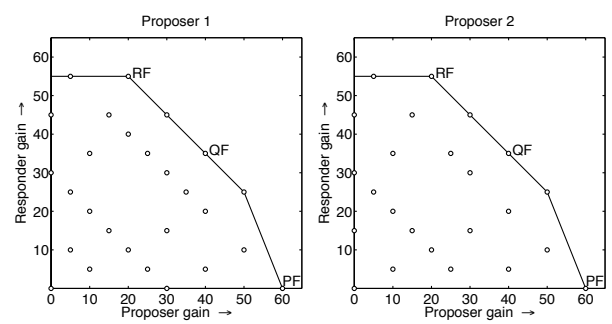

(b) Gain graph of the game instance presented in (a).
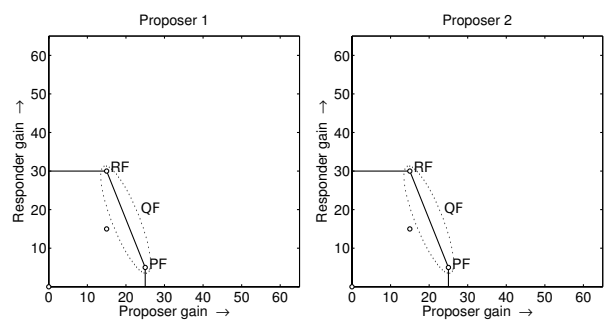

(d) Gain graph of the game instance presented in (c).

Figure 5.I: Example Colored Trails game instances and gain graphs. In (a) and (c), the three players $(R, P 1$ and $P 2)$ are shown, along with their chip sets. The two proposers cannot observe each others' chip sets. All players can see the board, on which their locations are indicated, as well as the goal state (a yellow flag). In (b) and (d), we show the gain graphs for both proposers. These graphs plot proposer gain versus responder gain for each possible proposal with non-zero benefit. The convex hull in this graph denotes the Pareto-front. The metastrategies PF, RF and QF are located on this front, as indicated. In (b), QF is a pure metastrategy; in (d), it is a mixed metastrategy (of PF and RF), since there is no proposal on the convex hull between PF and RF.

c) Response phase. The responder is presented with the two proposals. It can only accept one or reject both proposals and is not allowed to make a counter-proposal.

d) Termination and scoring phase. In this phase, players automatically exchange chips if they have reached agreement, and the icon of each player is advanced as close as possible towards its goal (using the Manhattan path with the shortest distance) given the result of the negotiation. The game ends and scores are automatically computed for each player: for every step between the goal and the player's position, 25 penalty points are subtracted. For every chip the player has not used, it receives Io extra points.

We use the following terminology associated with scores. First, the base score for a player $p \in\{R, P 1, P 2\}$ is the score the player receives when there is no agreement. ${ }^{2}$ Sec-

\footnotetext{
${ }^{2}$ Whenever we are not referring to one specific proposer, we will use the general notation ' $P$ ' when we imply ' $P 1$ and/or $P 2$ '.
} 
ond, the gain for a player $p$ and a chip exchange proposal $s$ denotes the difference of the final score given that $s$ is realized and the base score, and is denoted as $G_{p}(s)$. The base score for $p$, that is, the gain when there is no agreement, is denoted as $G_{p}(\emptyset)$.

For example, in Figure 5.I(a), the gain for the first proposer is $G_{P 1}(\emptyset)=-20$. If there is no agreement, the player can only move one square to the left by using its red chip. It is still two squares away from the goal, yielding $2 \times 25=50$ penalty points. It has 3 remaining chips, yielding $3 \times 10=30$ points. In this particular game, $G_{P 2}(\emptyset)=-20$ as well, with the optimal move being one to the right, using one red chip. The responder has a base score of -25 ; it can spend two blue chips to go right and upward, yielding a distance of 3 to the goal (75 penalty points) and 5 remaining chips (50 extra points). One possible proposal for $P 1$ is to offer a red and a grey chip for a blue chip, a green chip, and three yellow chips from the responder. In this case the proposer can get to the goal, and receives a gain of 6o. The responder can now reach a location only one square away from the goal at the expense of all its chips; thus resulting in a zero gain.

\subsection{Proposer Metastrategies}

Although the rules of the CT game are simple, it is not trivial to analyze. Both proposers need to reason about the tradeoff between making beneficial offers to the responder and offers that are beneficial for themselves Moreover, the number of possible strategies is large. In the example instance presented in Figure 5.I(a), the number of unique proposals for $P 1$ is 240 , while $P 2$ can choose from 144 unique proposals. ${ }^{3}$ The responder can choose to accept or reject any of these offers, so the size of the strategy space for the responder is $240 \times 144 \times 3=103,680$. The size of the combined strategy space makes it difficult to analyze this game in a principled way. In this section we show how to reduce this large setting to smaller interactions in a way that preserves the strategic flavor of the original CT scenario.

The analysis presented in this section is not specifically tailored to (three-player) CT. Any multi-agent one-shot negotiation setting may be analyzed in the manner presented here; as with CT, agents may each have a large number of actions to choose from, making straightforward game-theoretic analysis very hard. We will discuss this after outlining the analysis.

\section{Initial Assumptions}

We first describe two assumptions we make about the various players in the game. We will relax the first assumption later when we discuss social factors such as inequity aversion.

\footnotetext{
${ }^{3}$ Two proposals are unique if they do not use the same chips.
} 
Rational responder: The responder $R$ has three possible actions, i.e., to accept the proposal of $P 1$, to accept the proposal of $P 2$, or to accept neither of them. For the responder, the game is thus similar to an ultimatum game with proposer competition, see e.g., Hauert (20I0). Initially, in our analysis, we assume that the responder plays according to a rational strategy. If both proposals do not provide the responder with a positive gain, both proposals are rejected; if both proposals yield an equal gain, one proposal is accepted at random; if one proposal is strictly better then the other, it is accepted.

Semi-rational proposers: A strategy for the proposer player consists of an offer made to the responder. To choose a strategy, proposers have to take into account the gain resulting from each proposal for themselves as well as the responder. For our analysis, we assume that proposer $P$ limits the set of possible proposals to those that (a) lead to a non-negative personal gain, i.e., $G_{P}(s) \geq 0$, and (b) have a chance of being accepted by the responder, i.e., $G_{R}(s) \geq 0$. For example, in Figure 5.I(a), $P 1(P 2)$ has $79(50)$ valid proposals given this limitation.

\section{Analysis of Scenario}

In the two-proposer setting we consider, proposers are in competition with each other, which means proposers have to take into account the gain of the responder. To facilitate analysis, we plot the gains $G_{R}(s)$ against $G_{P}(s)$ for each possible proposal $s$ in a gain graph. Gain graphs for the two example games are given in Figures 5.I(b) and 5.I(d). In the interaction between a proposer and the responder, the Pareto-dominant proposals are located on the convex hull, as indicated in the figures. We note the following proposals located on the convex hull.

Proposer focus (PF): $P F$ is the strategy in which the proposer first maximizes its own gain, and then finds the maximum gain for the responder.

$$
P F_{P}=\underset{s^{\prime}}{\arg \max } G_{R}\left(s^{\prime}\right), s^{\prime} \in \underset{s}{\arg \max } G_{P}(s), s \in \mathscr{S}
$$

Responder focus (RF): $R F$ is the strategy in which the proposer first maximizes the responder's gain, and then finds the maximum gain for itself.

$$
R F_{P}=\underset{s^{\prime}}{\arg \max } G_{P}\left(s^{\prime}\right), s^{\prime} \in \underset{s}{\arg \max } G_{R}(s), s \in \mathscr{S}
$$

We call these proposals metastrategies, as their definition does not depend on the actual CT setting. The proposals corresponding to the metastrategies PF and RF for the example CT games appear in Figures 5.I(b) and (d). In the example instance of Figure 


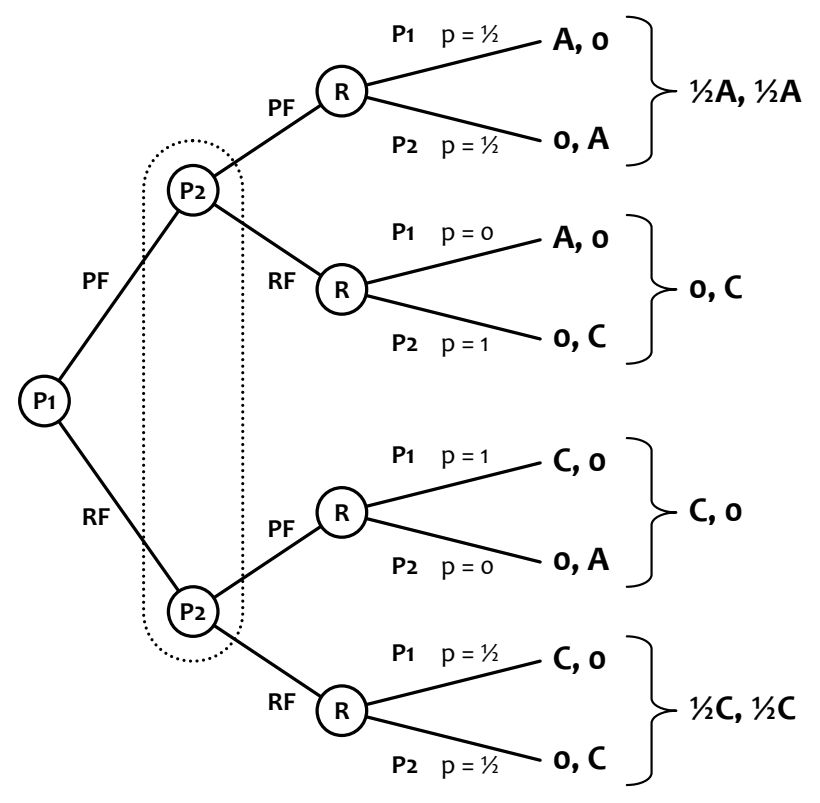

Figure 5.2: Extensive-form representation of the three-player negotiation variant of CT with two proposer metastrategies. The payoff for the rational responder $\mathrm{R}$ is not shown.

5.I(b), the strategy PF for PI corresponds to the chip exchange we mentioned before (in which PI offers one red chip and one gray chip in exchange for a blue, a green, and three yellow chips, leading to a gain, if accepted, of 60 for PI and 0 for R), while RF corresponds to giving a blue chip, a red chip, and a gray chip in exchange for two green chips (leading to a gain of 20 for PI and 55 for R here).

\section{Interactions between Metastrategies}

Suppose that proposers play only the metastrategies PF and RF. We show an extensiveform representation of the resulting CT scenario in Figure 5.2 (for proposer 2). We do not list the payoff for the responder from playing its rational strategy. In the figure, the two decision nodes of $P 2$ are grouped into one information set, because the players make their proposals simultaneously. Once $P 2$ has chosen, the static and rational strategy of the responder (which is indicated in the figure) leads to certain expected gains. ${ }^{4}$ Here, $A$ denotes the gain that a proposer receives when playing $\mathrm{PF}$ and being accepted; $C$ denotes the gain for RF being accepted. Clearly, this extensive-form game

\footnotetext{
${ }^{4}$ When calculating these expected gains, we assume that metastrategy pairs (e.g., $P F_{P 1}$ and $P F_{P 2}$ ) yield the same gain for the responder, so the responder is indifferent to the two proposals. In other words, we assume the CT game instance is symmetric. A CT game instance may generally not be (fully) symmetric, unless we explicitly generate only symmetric games. As we will discuss in our experimental section, using our set of criteria that lead to strategically interesting games, we find games that are symmetric in expectation, even though symmetry is not a criterium.
} 
Table 5.I: Induced two-proposer game with two metastrategies.

\begin{tabular}{ccc|}
\multicolumn{1}{c}{} & PF & RF \\
\cline { 2 - 3 } $\mathbf{P F}$ & $\frac{1}{2} A, \frac{1}{2} A$ & $0, C$ \\
$\mathbf{R F}$ & $C, 0$ & $\frac{1}{2} C, \frac{1}{2} C$ \\
\cline { 2 - 3 } & &
\end{tabular}

can be represented in a $2 \times 2$ matrix game, which omits the responder's strategy. The gain matrix of the symmetric game between the two proposers is shown in Table 5.I. Since the game between the two proposers is a $2 \times 2$ matrix game, it is straightforward to analyze. The game depends on the relationship between $A$ and $C$, as follows. For $A<2 C$, the game is a prisoners' dilemma, with one Nash equilibrium at (RF, RF) and a Pareto-optimal outcome at (PF, PF). For $A \geq 2 C$, we obtain a stag hunt game, with two pure Nash equilibria; the RF-equilibrium (shorthand notation) is risk-dominant, while the PF-equilibrium is payoff-dominant. The stag hunt game is another well-known social dilemma and differs from the prisoners' dilemma in that mutual cooperation is also a Nash equilibrium (Messick and Brewer, 1983).

The strategic qualities of the original CT game are preserved in the $2 \times 2$ matrix game played between metastrategies. In the original CT game, the RF metastrategy corresponds to offering the best possible offer to the responder. RF is therefore also the risk-dominant proposal in the original game, because it guarantees a positive gain to the proposer. Even if both proposers play RF, the expected gain for each proposer will be positive. In contrast, the PF metastrategy is payoff-dominant but risky, because it provides a low (or even zero) gain to the responder. It will yield the most positive possible gain (payoff) for the proposer if the other proposer also plays PF, but will yield no gain at all otherwise. The proposers' dilemma in the original game (favoring themselves or the responder) is thus accurately reflected in the reduced $2 \times 2$ matrix game.

In the example CT instance shown in Figure 5.I(a) and (b), we find that the PF strategy yields a gain to the proposer of 60 and a gain of 0 to the responder if accepted, while the RF strategy yields a gain of 20 to the proposer and 55 to the responder. Hence, $A=60$ and $C=20$ here, and $A>2 C$; the game played between the two proposers is thus a stag hunt. In a similar manner, we can conclude that the CT game of Figure 5.I(c) and (d) is a prisoners' dilemma, because $A=25$ and $C=15$ yields $A<2 C$.

\section{Introducing a Third Metastrategy}

While we distinguish only two metastrategies thus far, a proposer's actual strategy $s$ may be mixed, yielding (in theory) infinitely many possible (mixed) strategies $s$ based on the two metastrategies. 

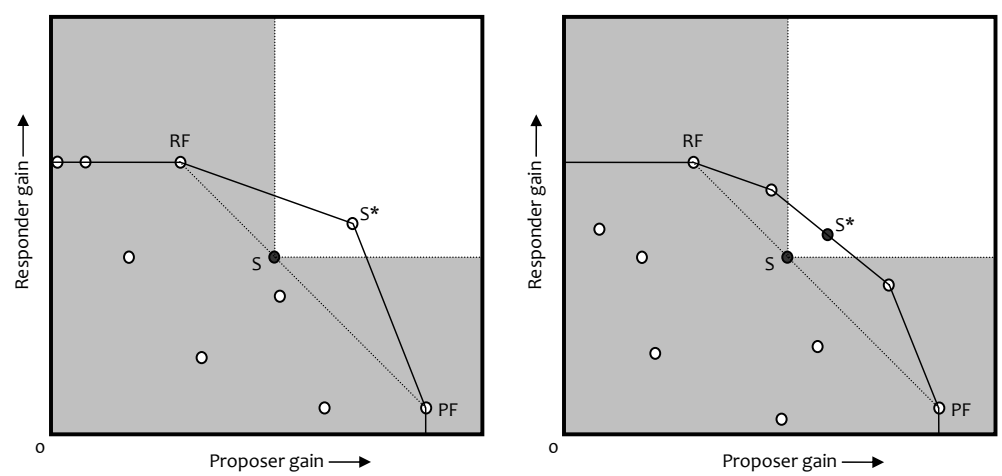

Figure 5.3: Pure or mixed strategies on the convex hull may strictly dominate a mixed strategy of PF and RF. In the example on the left, we find a pure strategy $s^{*}$ on the convex hull that strictly dominates a mixed strategy $s$ of PF and RF. On the right, a mixed strategy $s^{*}$ strictly dominates $s$.

We now demonstrate that a proposer can benefit from employing a metastrategy other than such a mixed strategy $s$. This is illustrated in Figure 5.3 (left and right). In the gain graph, all mixed strategies of PF and RF are located on the straight line connecting PF and RF. From the proposer's perspective, any mixed strategy $s$ is strictly dominated by a strategy $s^{*}$ for which $G_{P}\left(s^{*}\right)>G_{P}(s)$. This constraint is met by all points to the right of $s$ in the plot. Given that the responder behaves rationally, we say that $s^{*}$ strictly dominates $s$ if and only if $G_{R}\left(s^{*}\right)>G_{R}(s)$. All points above $s$ in the plot meet this constraint. Thus, strategies that lie in the unshaded area of the graphs in Figure 5.3 strictly dominate the mixed strategy $s$.

In some cases, the convex hull may lie on the straight line between PF and RF, as for instance in the second example game, see Figure 5.I(d); then, there is no strategy that strictly dominates $s$. In other cases however, as in Figures 5.3 and 5.I(b), the convex hull may be located above the line PF-RF. In these cases, we can always find a strategy $s^{*}$ that strictly dominates $s$, except if $s$ is a pure strategy itself, i.e., if $s$ assigns a probability of 1 to a certain metastrategy. For instance, in Figure 5.3 (left), we find a pure strategy $s^{*}$ on the convex hull that dominates $s$. In Figure 5.3 (right), a mixed strategy $s^{*}$ on the convex hull dominates $s$. In Figure 5.I(b), both proposers have three pure strategies (and an infinite number of mixed strategies involving one or more of these pure strategies) that dominate a mixed strategy of PF and RF.

Thus, proposers indeed may benefit from employing additional metastrategies, since these additional metastrategies may dominate (mixed strategies of) the two metastrategies we already defined. We therefore introduce a third metastrategy, named QF (where $\mathrm{Q}$ is chosen simply because it is between $\mathrm{P}$ and $\mathrm{R}$ ), which is to play the median proposal on the convex hull. Note that the median may be defined for any number of 
Table 5.2: Induced two-proposer CT game with three metastrategies.

\begin{tabular}{c|ccc|}
\multicolumn{1}{c}{} & $\mathbf{P F}$ & $\mathbf{Q F}$ & $\mathbf{R F}$ \\
\cline { 2 - 4 } $\mathbf{P F}$ & $\frac{1}{2} A, \frac{1}{2} A$ & $0, B$ & $0, C$ \\
$\mathbf{Q F}$ & $B, 0$ & $\frac{1}{2} B, \frac{1}{2} B$ & $0, C$ \\
$\mathbf{R F}$ & $C, 0$ & $C, 0$ & $\frac{1}{2} C, \frac{1}{2} C$ \\
\cline { 2 - 4 }
\end{tabular}

proposals on the convex hull; for an even number, we probabilistically select a proposal from the two median ones. Thus, proposals corresponding to the third metastrategy may again be found in any CT game.

Figures 5.I(b) and 5.I(d) show the mixed metastrategy QF for the two example CT instances. We see that the first instance has a pure QF metastrategy that dominates a mixed strategy of PF and RF. The gain graph shows that QF yields a proposer (responder) gain of 40 (35) here. The second instance has no strategies on the convex hull that dominate a mixed strategy of PF and RF; still, QF may be defined by choosing probabilistically from PF and RF. The (expected) gain for QF is then the average of the gains for $P F$ and RF, i.e., 20 for the proposers and 17.5 for the responder.

With three metastrategies, the game between the two proposers becomes a $3 \times 3$ matrix game, see Table 5.2. Note that $A \geq B \geq C$. It is straightforward to observe that potential equilibria are located on the diagonal of the matrix. Moreover, as in the twostrategy game, (RF, RF) is an equilibrium. Depending on the values of $A, B$, and $C$, we may distinguish four different games. For all games in which $A<2 B<4 C$, (RF, RF) is the sole equilibrium. For $A \geq 2 B \geq 4 C$, all three diagonal strategies are equilibria. For $A<2 B$ and $B \geq 2 C$, the equilibria are (RF, RF) and (QF, QF). For $A \geq 2 B$ and $B<2 C$, we find equilibria at (RF, RF) and (PF, PF).

In the example of Figure 5.I(a), we find $B=40$ ( $A=60$ and $C=20$ still holds); thus, $A<2 B$ and $B=2 C$, meaning the $3 \times 3$ matrix game has two equilibria, i.e., the RF- and the QF-equilibrium. In Figure 5.I(c), we find $A=25, B=20$ and $C=15$, so $A<2 B$ and $B<2 C$, yielding a single equilibrium at $(\mathrm{RF}, \mathrm{RF})$.

\section{Social Factors}

Thus far we have assumed the responder to be rational. Empirical evidence (in ultimatum game settings) suggests that human responders are actually not fully rational (Gintis, 200o). One of the most well-known alternative models for ultimatumgame responder behavior is inequity aversion (Fehr and Schmidt, I999). The responder does not act directly on its gain $G_{R}(s)$, but instead on a utility function $U_{R}(s)$, which depends on its own gain, but also on how it compares to the gain of the proposer, $G_{P}(s)$. The original model distinguishes two components in the utility function, namely greed 

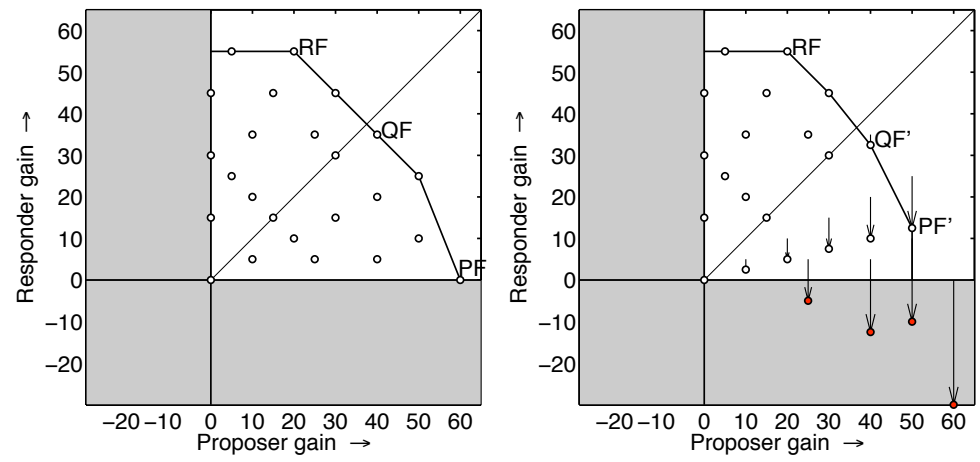

Figure 5.4: Effect of inequity aversion on the utility (perceived gain) of the responder: the convex hull and the metastrategies change. PF will no longer be accepted by the responder, which means proposers need to offer $\mathrm{PF}^{\prime}$ instead.

and compassion, both of which decrease the responder's utility in comparison to the actual gain. The greed component is generally far stronger (in humans); we do not consider the compassion component here. Translated to our settings, the responder's utility function may be then defined as follows.

$$
U_{R}(s)= \begin{cases}G_{R}(s) & G_{R}(s) \geq G_{P}(s) \\ G_{R}(s)-\alpha_{R}\left(G_{P}(s)-G_{R}(s)\right) & \text { otherwise }\end{cases}
$$

There is one parameter, $\alpha_{R}$, which determines how strongly the responder dislikes a proposal that gives a proposer more gain than the responder.

To illustrate the effect of inequity aversion, we apply it to the gain graph of proposer I in the first example game, see Figure 5.I(a) and (b). The effect for $\alpha_{R}=0.5$ is visualized in Figure 5.4. For proposals that give the proposer a higher gain than the responder (i.e., below the diagonal), the utility (perceived gain) for the responder is lower than the actual gain. As a result, some proposals that may be accepted by a rational responder are not accepted by an inequity-averse responder. As is clearly visible from Figure 5.4, the convex hull changes, as does the location of the PF metastrategy. If the responder is inequity-averse and the proposers do not take this into account, they may coordinate (without communication) to offer the PF proposal, expecting that one of them will be accepted, while in reality, the responder will reject both proposals.

Instead of offering PF, proposers should offer $\mathrm{PF}^{\prime}$, yielding a gain of 50 instead of 60 in the example. They are able to find $\mathrm{PF}^{\prime}$ if they are aware of the inequity aversion present in the responder (i.e., the value of $\alpha_{R}$ ), which implies they can calculate the modified gain graph. We deal with both unaware as well as aware proposers in our empirical evaluation (see Section 5.4). 


\subsection{Generalizing the Analysis}

In this section we show that our analysis may be generalized to more than three metastrategies and other variants of the CT game.

\section{Arbitrary Numbers of Metastrategies}

We can introduce additional metastrategies in a similar way to introducing a third metastrategy. For example, it is easy to include five metastrategies that correspond to the minimal, first quartile, median, third quartile, and maximal proposals on the convex hull. Generally speaking, for $n$ metastrategies, we obtain an $n \times n$ matrix game. The $n$ diagonal strategies may each be equilibria or not, except the ever-present RFequilibrium. Depending on the gains for each metastrategy pair, there are $2^{n-1}$ different possibilities for equilibria in this $n \times n$ game.

\section{Generalizing to Multiple Players}

The analysis above is specifically performed on the three-player negotiation variant of CT. However, results generalize to other games within the CT framework as chip exchanges are a vital part of the framework (Grosz et al., 2004).

I-proposer, I-responder: Another common variant of CT is a simpler two-player negotiation game, i.e., one proposer and one responder, potentially with multiple phases and/or alternating roles (Haim et al., 20Io). The one-shot two-player game also allows us to construct the gain graph and identify the metastrategies. Since the dilemma (and the associated competition) between the proposers is missing, the single proposer may get away with offering PF every time. The game is highly similar to the canonical ultimatum game. The optimal strategy $s$ for the proposer $P$ against a rational responder maximizes its gain while providing a non-negative gain for the responder (i.e., the optimal strategy is $\left.\arg \max _{s} G_{P}(s)\right)$.

If there is only one rational responder the outcome of the game is deterministic. The responder has full information about the state of the game and maximizes her reward. Lets assume accepting a RF offer results in payoff $X$ for the responder, accepting a PF offer results in payoff $Y<X$. The resulting payoff matrix is shown in Table 5.3 The rational responder always accepts and we clearly see that (PF, Accept) is the only Nash equilibrium. In games with an inequity averse responder the utility $U_{R}=Y-\alpha(X-Y)$ of the responder might drop below zero and thus may result in 'Reject' becoming the best response.

I-proposer, m-responders: In a game with multiple responders we can still construct gain graphs between pairs of proposer(s) and responders, with an ultimatum game with 
Table 5.3: Payoff matrix for induced CT game with one proposer and one responder.

\begin{tabular}{ccc|}
\multicolumn{1}{c}{} & PF & RF \\
\cline { 2 - 3 } Accept & $A, Y^{*}$ & $C, X$ \\
Reject & 0,0 & 0,0 \\
\cline { 2 - 3 } & &
\end{tabular}

Table 5.4: Payoff matrix for induced CT game with one proposer and $m$ responders.

\begin{tabular}{c|cc|}
\multicolumn{1}{c}{} & $\mathbf{P F}$ & $\mathbf{R F}$ \\
\cline { 2 - 3 } Accept & $A, \geq \frac{1}{m} Y$ & $C, \geq \frac{1}{m} X$ \\
Reject & 0,0 & 0,0 \\
\cline { 2 - 3 } & &
\end{tabular}

responder competition (Grosskopf, 2003) taking place between these pairs. Analytical and experimental studies in the ultimatum game clearly indicate that players benefit from an increased number of opponents in the opposite role, e.g., responders fare well with more proposers (Grosskopf, 2003; Hauert, 2010).

If there are multiple responders the game state is not fully observable. If a responder accepts a proposal she is no longer guaranteed to receive it as other responders might have accepted it as well. For $k$ responders that have accepted a proposal, the probability of a successful chip exchange for each responder is thus $\frac{1}{m}$. If the proposer offers $\mathrm{RF}$ each responder has an expected payoff of at least $\frac{1}{m} X$ (if all other responders accept as well, i.e. $k=m$ ) for accept and 0 for reject. If the proposer offers PF, each responder has an expected payoff of at least $\frac{1}{m} Y$ for accept and 0 for reject. The resulting game is shown in Table 5.4. Similarly, a rational responder prefers any guaranteed non-zero payoff over rejecting and thus accepts any offer. The Nash equilibrium for any I-proposer, m-responder game is thus (PF, Accept,...,Accept).

n-proposer, I-responders: The situation can be reversed to a game with multiple proposers and a single responder. This constitutes a social dilemma between the proposers, which can be modeled as a public goods game (Gintis, 200o) with ultimatumtype interactions between each proposer and the responder.

If all proposers play $\mathrm{PF}$, proposals are accepted with uniform probability resulting in expected payoffs $\frac{1}{n} A$ for proposers and $Y$ for the responder. If $C>\frac{1}{n} A$ then there is an incentive to deviate and proposers switch to RF. If there is at least one proposer playing $\mathrm{RF}$, all PF proposers receive $\mathrm{o}$ and thus have an incentive to switch to RF resulting in $\frac{1}{k} C>0$ where $k$ is the number of RF proposals. This behavior cascades and thus all proposers play RF.

For $A \geq n C$ this class of games has two equilibria, i.e. (PF,..,PF, Accept) and (RF,..,RF, Accept), and relates to a stag hunt game. The PF-equilibrium is payoff-dominant, while 
the RF-equilibrium is risk-dominant. For $A<n C$, games relate to a prisoners' dilemma with a single Nash equilibrium at (RF,...,RF, Accept). Note that the 3-player negotiation variant, as discussed extensively above, falls in this class of games.

n-proposers, m-responders: Outcomes for games with multiple proposers and multiple responders depend strongly on the rules that are established for assigning offers to players. For example, in a two-proposer, two-responder game, what happens if PI offers PF, P2 offers RF, RI accepts every positive offer, and R2 accepts only PF? Are offers then matched at random, or is there an algorithm that determines the optimal assignment? In the latter case, how do we define such an optimal assignment? Does fairness play a role here, too? In other words, establishing sensible rules for large-scale CT games is far from trivial, and these rules strongly influence the expected payoffs for certain (meta-)strategies. For this reason, we do not present an analysis of games with multiple proposers as well as multiple responders here.

\subsection{Empirical Evaluation}

In this section, we outline how strategically interesting instances of the CT game may be generated. We then discuss how players that perform actions according to the metastrategies may be heuristically implemented. Finally, we generate a large number of games that are played by these heuristic players. We evaluate the resulting empirical payoff tables and compare the results with the preceding analytical analysis.

\section{Strategically Interesting Games}

Strategically interesting games, i.e., games that require strategic thinking from their players, are fair games that require negotiation and mutual dependence.

Fair baseline scores: The initial board state (positions and chip sets) should yield baseline scores that are comparable for all three players. We generate games that limit the difference in baseline scores to be less than a certain $\epsilon$.

$$
\begin{aligned}
& \max \left\{G_{P 1}(\emptyset), G_{P 2}(\emptyset), G_{R}(\emptyset)\right\} \\
- & \min \left\{G_{P 1}(\emptyset), G_{P 2}(\emptyset), G_{R}(\emptyset)\right\}<\epsilon
\end{aligned}
$$

Negotiation requirement: No player should be able to reach the goal location on its own without engaging in a chip trade. We define isSolution $(P, C)=$ true iff player $P$ can reach the goal given a chip set $C$. The initial chip set of a player $P$ is given by $\operatorname{chips}(P)$. 
The negotiation requirement is:

$$
\begin{aligned}
& \neg i s \text { Solution }(P 1, \operatorname{chips}(P 1)) \wedge \\
& \neg i s \text { Solution }(P 2, \operatorname{chips}(P 2)) \wedge \\
& \neg i s \operatorname{Solution}(R, \operatorname{chips}(R))
\end{aligned}
$$

Mutual dependence: Due to the negotiation requirement, both proposers depend on a subset of the responder's chip set. In turn, the responder relies on a subset of either proposer I or proposer 2. A one-sided proposal (i.e. asking for all chips or dispensing of all chips) may not lead to a chip set allowing both the proposer and the responder to reach the goal.

$$
\begin{aligned}
& \exists C_{P 1}, C_{R} \in \operatorname{chips}(P 1) \cap \operatorname{chips}(R) \text { s.t. } \\
& \quad C_{P 1} \cap C_{R}=\emptyset \wedge \text { isSolution }\left(P 1, C_{P 1}\right) \wedge \operatorname{isSolution}\left(R, C_{R}\right) \\
& \exists C_{P 2}, C_{R} \in \operatorname{chips}(P 2) \cap \operatorname{chips}(R) \text { s.t. } \\
& \quad C_{P 2} \cap C_{R}=\emptyset \wedge \text { isSolution }\left(P 2, C_{P 1}\right) \wedge \operatorname{isSolution}\left(R, C_{R}\right)
\end{aligned}
$$

We implement these three criteria by generating many pseudo-random games and checking them against the criteria, keeping only those games that match. In a similar manner, we may introduce additional criteria, such as symmetry (see the discussion following).

\section{Experimental Setup}

For the empirical evaluation of the proposed metastrategies we generate a database of 10,000 games that adhere to the criteria listed above (we chose $\epsilon=20$ ). Below, we discuss how the metastrategies are implemented in heuristic players and how empirical payoffs are computed from games played between these players.

Heuristic players: We implemented three heuristic players, each following one of the three metastrategies, i.e. PF, QF and RF. All three heuristic players start by enumerating all possible chip exchange proposals. Proposals that yield negative gains for either the proposer or the responder are neglected. Heuristic players following metastrategies PF and RF are a straightforward implementation of the definitions given earlier. Metastrategy QF requires to compute the PF and RF strategy points in the gain graph, as well as the convex hull connecting both.The median proposal on the convex hull is then selected. For an even number, the heuristic player probabilistically selects a proposal from the two median ones. 
Table 5.5: Empirical payoff matrix for the CT game with two metastrategies.

\begin{tabular}{|c|c|c|}
\hline & PF & $\mathrm{RF}$ \\
\hline PF & $21.0,20.8$ & $2.9,11.8$ \\
\hline RF & $11.8, \quad 2.9$ & $6.5,6.3$ \\
\hline
\end{tabular}

Table 5.6: Empirical payoff matrix for the CT game with three metastrategies.

\begin{tabular}{|c|c|c|c|}
\hline & PF & QF & $\mathrm{RF}$ \\
\hline $\mathrm{PF}$ & $21.0,20.8$ & $5.7,24.3$ & $2.9,11.8$ \\
\hline $\mathrm{QF}$ & $24.5, \quad 5.6$ & $14.8,14.2$ & $6.0, \quad 9.8$ \\
\hline $\mathrm{RF}$ & $11.8, \quad 2.9$ & $10.0, \quad 5.7$ & $6.5, \quad 6.3$ \\
\hline
\end{tabular}

Computing empirical payoffs: A single entry of the empirical payoff matrix is computed as follows. The row determines the metastrategy played by PI, while the column determines the metastrategy for $\mathrm{P}_{2}$. For each game in the database, chip exchanges proposed by the players are evaluated by the responder and if a proposal is excepted, chips are exchanged and scores evaluated. The resulting payoff is the difference between final and baseline scores (i.e. gain) averaged over all 10,000 games and Io runs. This process leads to a full empirical payoff table for the game as a whole.

\section{Results}

In this section, empirical payoff tables obtained by the metastrategies are presented and compared to the predicted payoff tables.

With two metastrategies PF and RF, we obtain an empirical payoff table as shown in Table 5.5. The empirical payoffs yield a stag hunt game, with $A \approx 42$ and $C \approx 12$. When we compare the empirical payoff table to the analytical one, we note the following: First, the game is nearly, but not completely symmetric. This may be explained by the relatively small size of the board, which leads to relatively large differences (i.e. possible imbalances) between the two proposers. On the small board we use, symmetry arises from repeated play. The game is guaranteed to be symmetric in expectation, since proposers' positions are randomized. Second, there are (small) positive values where we expected values of 0 . In some instances, a certain proposer's PF proposal is preferred by the responder over the other proposer's RF proposal. Once again, this issue may be dealt with by using larger boards, which would reduce the probability that PF 'wins' from RF. However, larger boards are (even) more difficult for human players. 
Table 5.7: Empirical payoff matrix with inequity aversion (unaware proposers).

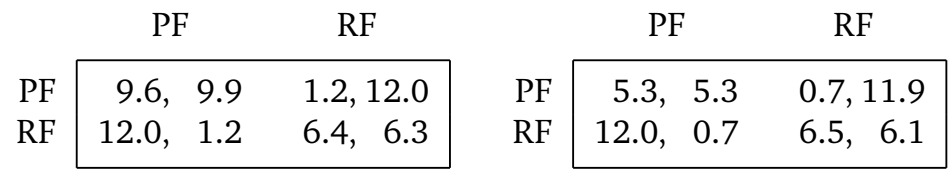

$$
\begin{aligned}
& \alpha_{R}=0.5 \quad \alpha_{R}=1.0
\end{aligned}
$$

Table 5.8: Empirical payoff matrix with inequity aversion (aware proposers).

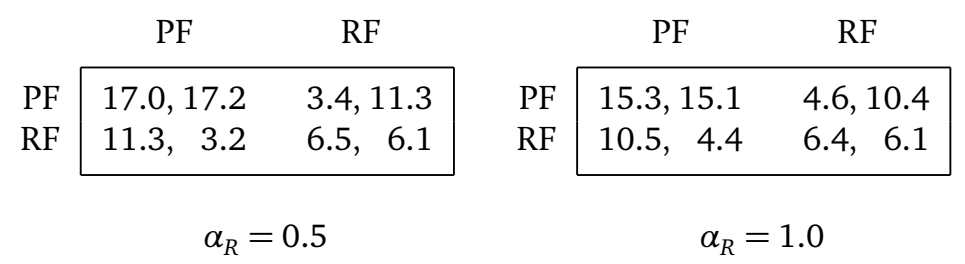

The empirical payoff table for the three-strategy game is given in Table 5.6. The values in the corners of the table are identical to those in the two-strategy game. We see that $B \approx 25$. It is interesting to consider the interactions between the 'neighboring' metastrategies. Looking at the interaction between PF and QF, we find a prisoners' dilemma. The QF metastrategy is very strong against PF, giving proposers a strong incentive to defect. Between QF and RF, we find a stag hunt. The payoff table thus yields a game with two pure equilibria, namely the QF- and the RF-equilibrium.

We now determine the effect of introducing social considerations (inequity aversion) in the responder's decision-making, without the proposers being aware of this. We provide the empirical payoff matrices for two reasonable values of $\alpha_{R}$ in Table 5.7, restricting ourselves to the two-strategy game. The second equilibrium (PF, PF) disappears, because proposers expect their PF proposal to be accepted more than it actually is. The game thus turns into a prisoners' dilemma with one Pareto-dominated equilibrium at (RF, RF) for $\alpha_{R}=0.5$. The higher the value of $\alpha_{R}$, the weaker strategy PF gets. For $\alpha_{R}=1.0, \mathrm{RF}$ is Pareto-efficient.

We also investigate what happens if the proposers do know that the responder is inequity-averse. The payoff matrices for the same values of $\alpha_{R}$ are given in Table 5.8. The second equilibrium is back again; proposers appropriately adjust their PF proposals to the expectations of the responder. The payoff for PF is (sensibly) lower against itself than in the original game with a rational responder. PF does increasingly well against RF, simply because PF is (slightly) more similar to RF when proposers take into account the responder's expectation. 


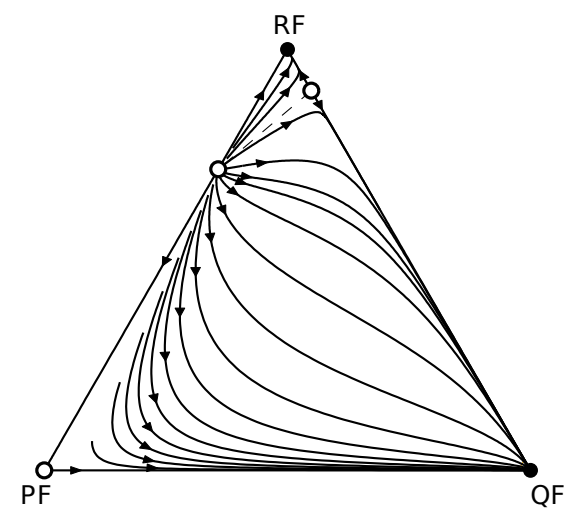

Figure 5.5: Single population dynamics in the three-strategy game. Evolutionarily stable strategy (ESS) attractors are depicted by filled circles; unstable rest points are shown as open circles. The dashed line marks the boundary between the basins of attraction.

\subsection{Evolutionary Analysis}

We will now proceed to analyze the empirical payoffs reported in the previous section. In particular we will take a closer look at the three-strategy game using tools from evolutionary game theory.

The replicator dynamics for the three-strategy game can be best visualized by a 2 simplex, this makes it possible to graphically and analytically study the dynamics of the game. Figure 5.5 shows the three dimensional strategy space projected on a equilateral triangle (2-simplex); the dimensionality is reduced by one as the state $\pi$ of the system is a probability vector.

Trajectories show the evolution of strategies according to the replicator equations: an equilibrium to which trajectories converge is known as an attractor; rest points that show diverging trajectories are called repellers; rest points with some trajectories approaching and others diverging are named saddle points. The strategy space that is consumed by an attractor is called the basin of attraction.

The game has two evolutionarily stable attractors corresponding to the pure strategy profiles RF and QF. The Nash equilibrium mixing between RF and PF is a repeller, while the mixed equilibrium between $\mathrm{RF}$ and $\mathrm{QF}$ is a saddle point. $\mathrm{PF}$ is a saddle point as well; the trajectory from the mixed Nash equilibrium between RF and PF converges to PF and PF itself is clearly dominated by QF. The basin of attraction of the QF attractor consumes by far more strategy space than the RF attractor. This result does not come as a surprise as we see a stag hunt game between QF and RF with QF yielding higher payoffs. While PF is Pareto efficient, it is dominated by QF. 

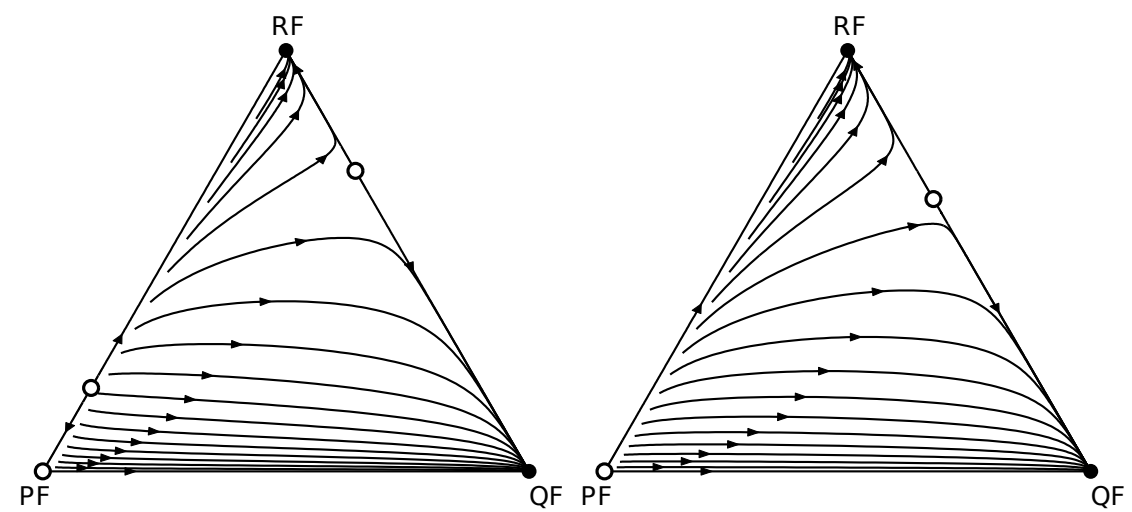

Figure 5.6: Single population dynamics in the three-strategy game with inequity aversion (responder only); $\alpha_{R}=0.5$ (left), $\alpha_{R}=1.0$ (right). Evolutionarily stable strategy (ESS) attractors are depicted by filled circles; unstable rest points are shown as open circles. The dashed line marks the boundary between the basins of attraction.

For games with inequity averse responders, the basin of the RF-equilibrium increases, as shown in Figure 5.6. If $\alpha$ is increased to I, the mixed equilibrium between RF and PF disappears and PF becomes a repeller. Even with inequity aversion QF still performs strong agains RF while dominating PF.

\subsection{Discussion}

Adding social factors to the responder allows this player to enforce a higher payoff essentially, the proposers are driven to defection in the stag hunt or prisoners' dilemma game they play, because the responder is better at exploiting the proposer competition in the ultimatum game component. This increased power for the responder may be countered by introducing multiple responders, as in an ultimatum game with responder competition (Grosskopf, 2003).

As noted, our analysis of a single game instance assumes that responders are indifferent between the gains from the two proposals resulting from any pair of metastrategies, i.e., for the metastrategies $P F_{P 1}$ and $P F_{P 2}$, we have that $G_{R}\left(P F_{P 1}\right)=G_{R}\left(P F_{P 2}\right)$ (and similar for $\mathrm{QF}$ and RF). If this condition does not hold, the responder will favor one of the metastrategy proposals over the other, which means the actual game instance does not reduce to a stag hunt or prisoners' dilemma. We observed that approximately $25 \%$ of the 10,000 games we generated (and that met our three criteria) were actually games in which the responder is indifferent between metastrategy pairs. Of these $25 \%$, approximately one-fifth are prisoners' dilemmas, and four-fifth are stag hunts. The remaining games (i.e., 75\%) were not symmetric, meaning that one proposer has 
a strategic advantage over the other proposer. Even though our symmetry assumption thus does not hold for a majority of generated game instances, our empirical results show that, even for games in which the assumption does not hold, the expected gains to proposers from playing metastrategies do in fact correspond to stag hunt and prisoners' dilemma games.

In case a certain experiment requires all games to be stag hunts or prisoners' dilemmas (i.e., not only in expectation), the assumption of responder indifference can be enforced during game generation. We note that, for the case in which responders are assumed to be rational, we do not need to make assumptions about the gains to proposers from pairs of metastrategies (a rational responder does not consider those gains), while for inequity-averse responders, the gains to proposers for every metastrategy pair must also be equal, i.e., $G_{P 1}\left(P F_{P 1}\right)=G_{P 2}\left(P F_{P 2}\right)$ (and similar for $\mathrm{QF}$ and $\mathrm{RF})$.

\subsection{Summary}

In this chapter we have shown how CT games can be decomposed into a set of multiple normal-form games that are characterized by social dilemmas, i.e., prisoners' dilemma, stag hunt and ultimatum games, using a number of metastrategies defined on the chip exchange proposals in the game. We show that the metastrategy that favors the responder (RF) is always an equilibrium and ESS in the reduced normal form game, regardless of the number of metastrategies. This is because the responder has an advantage in the CT setting we consider, in that no player receives a gain if it does not accept an offer. An empirical analysis of a large set of game instances illustrates that, in expectation, two metastrategies yield two equilibria, reducing it to a stag hunt game. We may also find game instances that are prisoners' dilemmas, i.e., with only the equilibrium that favors the responder. Using three metastrategies in the same set of game instances also yields two equilibria (both ESS), namely those two metastrategies that are most favorable for the responder ( $\mathrm{QF}$ and $\mathrm{RF}$ ). The evolutionary game-theoretic analysis of the three-strategy game corresponds with the analytical findings and agree with our claim that metastrategies allow us to decompose the CT game into bilateral social dilemmas. 


\section{6 \\ The Value of Information}

Markets play a central role in today's society, and range from stock markets to consumerto-consumer e-commerce (Angel, 2002; Bajari and Hortacsu, 2003). Economic theory often starts from perfect competition as an idealized assumption about markets. It relies, among other characteristics, strongly on a symmetric information structure. All traders have access to the same information about price and quality of goods. Many, if not all, of todays markets do not meet this utopian assumption and thus valorize the access to information. Undoubtedly, information is an important factor that has influence on trading success or losses. Insiders are clearly able to use their information to outperform the market. However, the relation between information level and success is not trivial.

Market forecasters and fund managers are generally assumed to be well informed, though for the most part they perform below market average. Cowles (I933) has been the first to study this phenomenon and reports that a group of trained forecasters performed $4 \%$ below market average during a period of 4.5 years. These findings have since been confirmed by multiple studies; for an overview we refer to Kirchler (2010) and Tóth et al. (2007), in particular we like to highlight that Malkiel (2003) reports on returns of actively managed funds over a period of 30 years - less then $15 \%$ of the funds outperformed the market.

Tóth et al. (2007) study the relation between information and performance for traders with various information levels both in simulation and in human experiments. Average-informed traders perform below market level, while non-informed traders reach the market average; highly informed traders beat the market. These results suggest that if a trader has no inside information, trading based on current market prices (non-informed) is most sensible. Relying on outdated or average information has a negative impact on returns.

Huber et al. (2008) and Kirchler (2010) investigate whether this negative impact can be explained by behavioral patterns. In particular, the authors test the hypothesis that low performance of average-informed traders is the result of overconfidence, i.e. over- 
estimating the value of (possibly outdated) information. Results show that traders do not exhibit overconfidence and low returns are caused by the asymmetric information structure itself. Huber (2007) offers the following explanation: during trends, foresight is clearly advantageous. When the trend reverses, the averagely informed trader trusting its information performs worst due to outdated information. Non-informed (random) traders are safe from these systematic mistakes.

The vast body of previous work (Huber, 2007; Huber et al., 2008; Kirchler, 2010; Tóth and Scalas, 2007; Tóth et al., 2006, 2007) has evaluated the advantage of information in markets from various perspectives. However, only markets with a limited number of traders and uniformly distributed information levels have been considered, and information was assumed to be free. Our work investigates several more realistic information distributions in larger markets. Furthermore, we extend the analysis by studying an infinite population of traders using an evolutionary model and demonstrate the influence of the price of information on market dynamics.

The remainder of this chapter is structured as follows: The following section provides background information on auctions, the value of information in auctions and existing evolutionary models of auctions. Section 6.2 introduces the market model and evaluation method used in the experiments. Evolutionary simulations and results are described and discussed in Section 6.3. Finally, Section 6.4 concludes the chapter.

\section{I Auctions and the Value of Information}

\section{Auctions}

Auctions are highly efficient match making mechanisms for trading goods or services. As such, they are employed by a number of real markets, such as telecommunication spectrum rights auctions or the New York Stock Exchange (NYSE) (Angel, 2002; McMillan, I994). In practice, there is a variety of rules that may be used to conduct an auction. Each set of rules may result in different transaction volumes, transaction delays, or allocative market efficiency. One sided auctions, especially with one seller and many potential buyers, are popular in consumer-to-consumer e-commerce (Bajari and Hortacsu, 2003; Barrot et al., 2010). Here, we focus on double auctions, which essentially provide a platform for buyers and sellers to meet and exchange a commodity against money. A taxonomy of double auctions especially tailored to automated mechanism design can be found in (Niu et al., 20I2).

Double auctions maintain an open book of bids (offers to buy at a specified price) and asks (offers to sell at a specified price). Two principle forms are the clearing house auction and continuous operation auction. In a clearing house auction, orders are collected for a trading period (e.g., one day) and matched, or cleared, after the trading 
period is closed. This mode of operation allows for high allocative efficiency, but incurs delays in the transactions. In contrast, continuous operation immediately establishes a transaction as soon as some buyer is willing to pay more than a seller is asking for. This mode allows higher transaction rates at the cost of some allocative efficiency. Experiments in this chapter will use continuous operation mode, since it reflects the day-time operation mode of the NYSE (Angel, 2002).

\section{Value of information}

It is common sense that training and additional information should increase performance for any task. However, the value of information in markets is non-monotonic, i.e., having some information may be worse than having none.

In order to measure the value of information, experiments in auctions measure revenue. Since revenue is heavily dependent on market conditions dictated by the price signal, it is normalized to reflect the relative return. Assume trader $i$ receives revenue $r_{i}$. The average profit $r_{a v g}=\frac{1}{n} \sum_{i} r_{i}$ in an $n$-trader market is used to compute the relative market return $u_{i}=\frac{r_{i}}{r_{\text {avg }}}-1$ for each trader.

\section{Dividend Discount Model}

The different information levels are implemented by varying the amount of knowledge that traders have about future dividends. In general, a trader with information level $I_{j}$ knows the dividend of this and the next $(j-1)$ periods.

Let us assume that we require a certain rate of return $r>0$ on our investment. For example, if $r=0.005$, a share must return $0.5 \%$ per trading period for it to be a worthwhile investment. Rate $r$ is also called the discount rate. A future dividend $D_{t}$ at time $t$ has a current discounted value of $\frac{D_{t}}{(1+r)^{t}}$. If we intend to hold the share indefinitely, the value of a share is equal to the sum of future discounted dividends:

$$
V=\sum_{t=1}^{\infty} \frac{D_{t}}{(1+r)^{t}}
$$

Gordon's growth model Gordon (I962) assumes that dividends grow at a constant rate $g$. If $D_{0}$ is the current dividend payout, the current stock value can be computed as follows:

$$
V=\sum_{t=1}^{\infty} D_{0} \frac{(1+g)^{t}}{(1+r)^{t}}=D_{0} \frac{1+g}{r-g}
$$

Let us assume the dividends are constant over time, i.e. $\forall i: D_{t}=D$ and $g=0$. The 
stock value simplifies to:

$$
V=\sum_{t=1}^{\infty} \frac{D}{(1+r)^{t}}
$$

The infinite series (6.2) converges to $\frac{D}{r}$ as $\frac{1}{|1+r|}<0$ with $r>0$. For example, a stock that pays a constant dividend of 0.2 per share has a current value of $V=0.2 / 0.005=40$.

\subsection{Market Model}

In order to analyze the advantage of foresight, we simulate a stock market in which traders with different amounts of information on future prices, or information levels, trade a certain asset. We closely follow the market model as described by Huber et al. (2008) and Tóth and Scalas (2007) in order to be comparable. The market is based on a continuous double auction with open order book, in which all traders can place bids and asks for shares. The intrinsic value of the shares is determined by a dividend stream that follows Brownian motion:

$$
D_{t}=D_{t-1}+\epsilon
$$

where $D_{t}$ denotes the dividend in period $t$ with $D_{0}=0.2$, and $\epsilon$ is a normally distributed random term with $\mu=0$ and $\sigma=0.01$, i.e., $\epsilon \sim \mathscr{N}(0,1)$. Figure 6.I shows an example dividend stream.

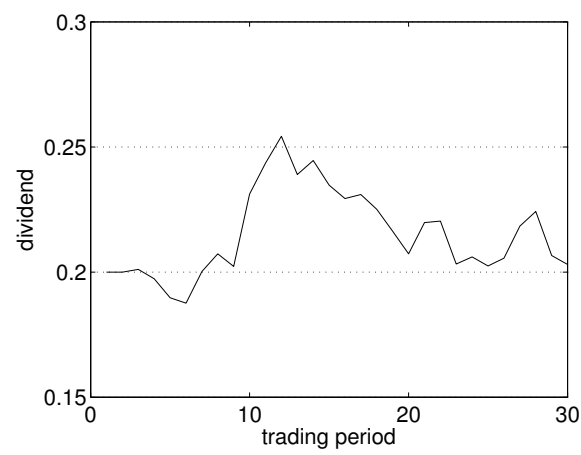

Figure 6.I: A Brownian motion dividend stream.

We simulate the market over 30 trading periods, each lasting $10 \cdot n$ time steps, where $n$ is the number of traders present. All traders start with r6oo units cash and 40 shares, each worth 40 in the beginning. At the beginning of each period, all traders can put a bid or ask in the book (opening call). Hereafter, at every time step a trader is selected at random who can then place a bid or ask according to its trading strategy (see below). 


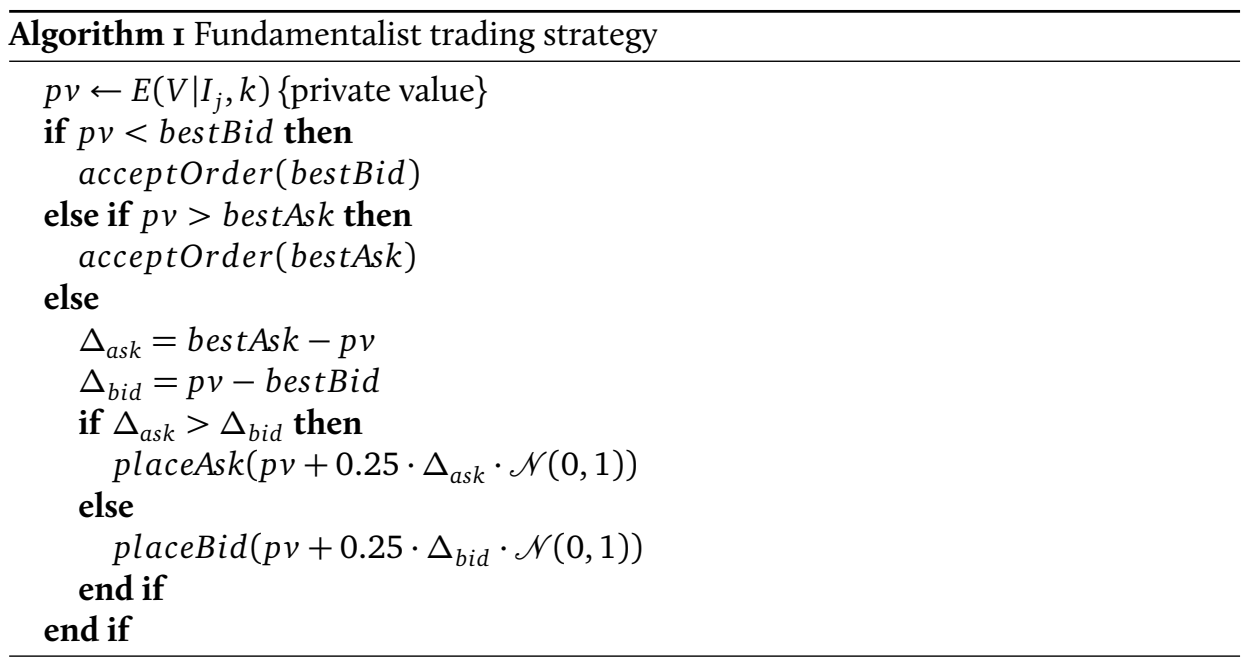

At the end of each period, dividend is paid based on the shares owned, and risk free interest rate (o.I\%) is paid over cash. The performance of the traders is measured as their total wealth after the 30 periods, i.e., each share is valued according to the discounted future dividends (see below) and added to the cash reserves.

The different information levels are implemented by varying the amount of knowledge that traders have about the future dividends. In general, a trader with information level $I k$ knows the dividend of this and the next $(k-1)$ periods. Traders with information level $I 0$ have no information about dividends and can only observe the current market price. This results in a cumulative information structure, where insiders know at least as much as averagely informed traders. The information that the traders receive each period is the conditional present value of the shares, conditioned on their information level. This value can be calculated using the dividend discount model (Gordon growth model) as

$$
E\left(V \mid I_{j}, k\right)=\frac{D_{k+j-1}}{\left(1+r_{e}\right)^{j-2} r_{e}}+\sum_{i=k}^{k+j-2} \frac{D_{i}}{\left(1+r_{e}\right)^{i-k}}
$$

where $V$ denotes the value, $I_{j}$ is the information level, $k$ the period, and $r_{e}$ the riskadjusted interest rate (set to $0.5 \%$ in our experiments).

\section{Trading strategies}

We use two different trading strategies in our experiments. Traders that have at least some information about the dividend stream (I1 and higher) use the fundamentalist strategy, that takes this information into account. Traders without any information (I0) use the random strategy, in which their bids and asks are based purely on the cur- 


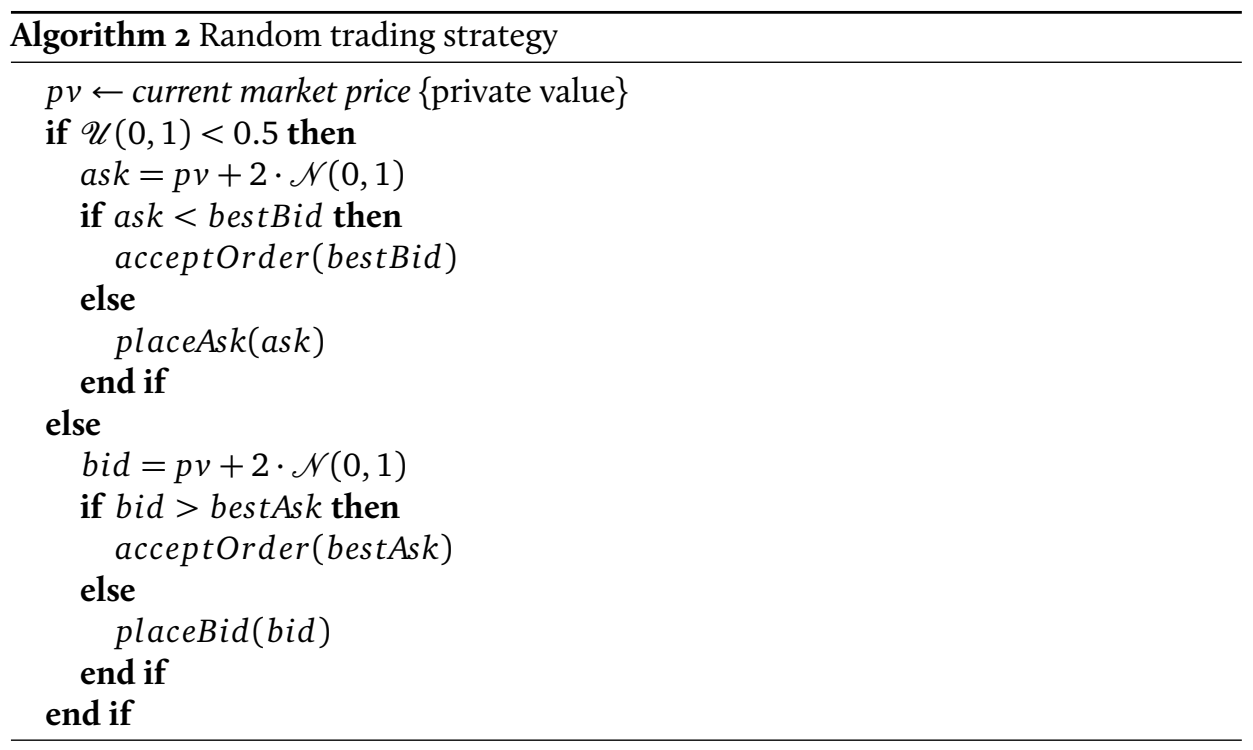

rent market price of the shares.

\section{Fundamentalists}

Fundamentalists completely rely on the information they receive. The fundamentalist strategy is explained in Algorithm I (see also (Tóth and Scalas, 2007; Tóth et al., 2006)). In essence, they compare their estimated present value $E\left(V \mid I_{j}, k\right)$ with the current best bid and ask in the book. If they find a bid (ask) with a higher (lower) value than their estimate, they accept the offer. Otherwise, they place a new order between the current best bid and ask prices. Naturally, the trader should own enough shares or cash to accept or place an order.

\section{Random traders}

The random trading strategy only takes the current market price into account when deciding whether to accept or place an order. With equal probability the trader sells or buys shares. The random trading strategy is explained in Algorithm 2 (see also (Tóth and Scalas, 2007; Tóth et al., 2006)).

\section{Simulations and results}

We simulate the market with varying numbers of traders for each information level, in order to analyze the relative performance of traders with different amounts of foresight. To reduce the effect of randomness we run Ioo sessions of Ioo simulations each; the dividend stream is fixed for each session. Results are given as the relative performance with respect to the market average plotted against the information levels. 


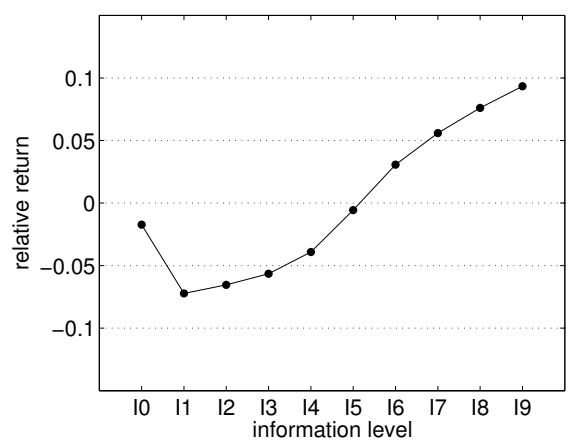

Figure 6.2: Relative market return over information level. Io traders with information level o to 9 (I trader for each level).

Figure 6.2 shows the results for a market of Io traders in Io information levels: one random trader, $I 0$, and 9 fundamentalists, $I 1 \ldots I 9$. As can be seen, performance does not necessarily increase with more information: the random trader performs at market average, whereas traders with limited amounts of information do significantly worse. Only highly informed traders are able to beat the market.

This result is in line with related work, where a similar shaped J-curve was reported (Kirchler, 2010; Tóth and Scalas, 2007). This relation between information level and performance, where more information is not always better, has also been observed in market experiments involving human traders (Huber et al., 2008). A possible explanation is that random traders are by definition not predictable, and therefore hard to exploit by insiders. On the other hand, experts can more easily predict and exploit traders with limited or average information levels.

Previous work has mainly focussed on small scale markets, with uniform and static distributions of traders over information levels (Huber, 2007; Huber et al., 2008; Kirchler, 20Io; Tóth and Scalas, 2007; Tóth et al., 2006, 2007). We believe that this may lead to an overly simplified model of reality, which may in turn influence the reported findings. For example, a market will be more likely to contain only a small number of insiders, and a large group of averagely informed traders. Furthermore, having only one trader per information level rules out within-group trading, which could bias results.

We extend the experiments of (Kirchler, 20I0; Tóth and Scalas, 2007) by looking at markets with more traders and non-uniform distributions of traders over information levels. An overview of relative market returns for a selection of information distributions is given in Figure 6.3. Next to the uniform distribution used in previous work, we run simulations with a normal distribution and a power-law distribution over information levels. These distributions are chosen to reflect information distributions that 

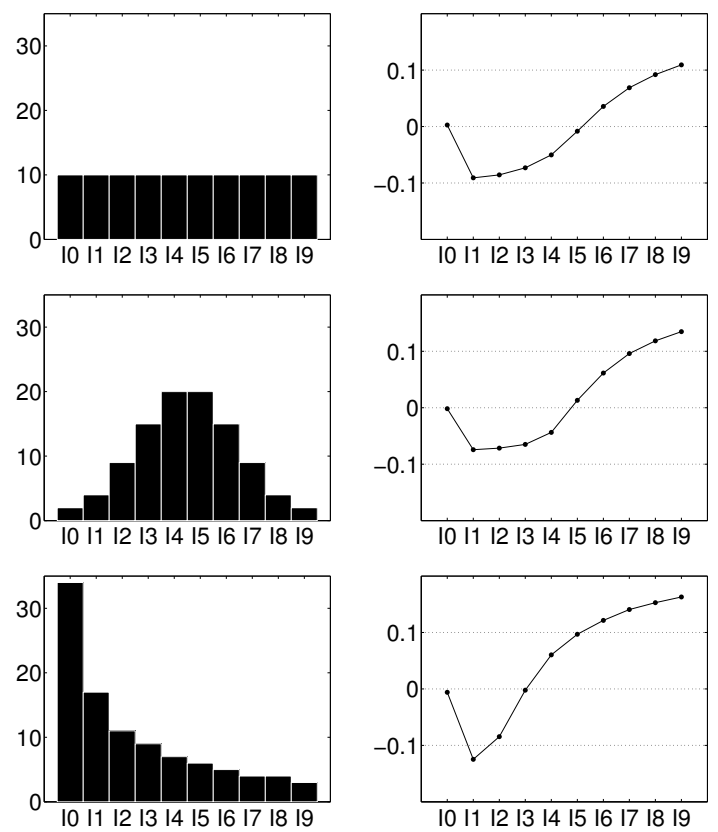

Figure 6.3: Relative market return over information level (right) for various information distributions (left) given a finite population of 100 traders.

are likely to be found in real markets. It is impossible to observe these distributions directly as the information level is private to the trader (Huber et al., 2008). The two chosen distributions follow from natural assumptions: (I) normal distributions arise if access to information is cumulative based on independent and identically distributed bits of information; (2) the power-law distribution is motivated by an information flow in scale-free social networks where every trader has access to information of his social ties.

As can be seen from this figure, random traders perform at market average under all three distributions, and traders with limited information underperform the market. However, the shape of the curve does change considerably depending on the information distribution. Where in the uniform scenario only traders with information level I6 or higher outperform the market, for the normal distribution this is the case for I5 and for the power-law distribution for I4. However, we can conclude that the J-curve is relatively insensitive to changing information distributions and numbers of traders. Only in extreme cases (not shown here) does the curve change drastically.

Figure 6.4 shows that relative market returns follow the J-curve even in small markets with only three information levels: random traders, averagely informed traders, and insiders. Again, this is in line with previous work, where a similar setup was shown 


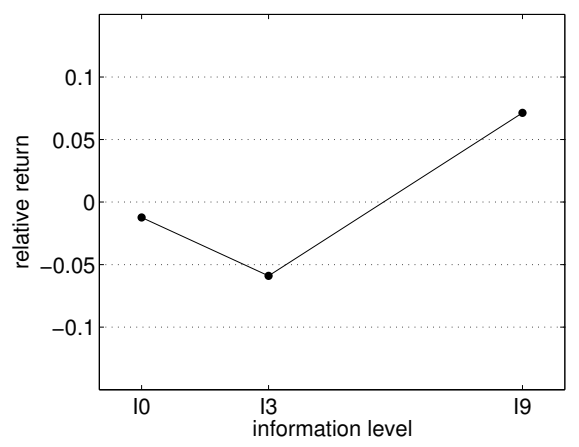

Figure 6.4: Relative market return over information level; I trader for each of the information levels 0,3 , and 9 .

to reflect stylized facts such as autocorrelation observed within real markets (Tóth et al., 2007). Note that the obtained curve does not change qualitatively when varying the information level of average-informed traders: any choice between $\{I 0, I 1, I 9\}$ and $\{I 0, I 8, I 9\}$ results in a J-curve.

\section{Discussion}

We have reproduced the J-curve of relative market returns over information levels that has been observed in previous work. Furthermore, we have shown that the specific shape of this curve prevails if the distribution over information levels changes. This indicates that the conclusions drawn from this may hold under more realistic settings as well.

This perspective still assumes that the distribution over information levels does not change over time. There are several ways this assumption may be violated in practice: First, traders may choose to acquire more information. For example, traders may or may not subscribe to financial news sources, which in turn determines their information level - possibly at a cost. The effect of having traders choose between trading strategies is investigated in (Kirchler, 2010; Tóth and Scalas, 2007), with the conclusion that only highly informed traders will choose their fundamental strategy, taking their information into account. Second, traders may take over a larger market share due to their financial success while others are driven out of the market. This motivates the evolutionary analysis that accommodates evolving distributions over information levels, and elicits the market dynamics. 


\subsection{Evolutionary Analysis}

The previous section provides a method to compute expected relative market revenues for selected information distributions. This views information distributions as isolated and fixed in time. However, the market revenue can be interpreted as Darwinian fitness, such that traders performing below market average should be driven out of the market, while those with higher returns prevail. This section will first introduce the evolutionary analysis methodologically, list the results and discuss their implication.

\section{Method}

The evolutionary model assumes an infinite population. We cannot compute the payoff for such a population directly, but we can approximate it from evaluations of a finite population.

All possible distributions over $k$ information levels can be enumerated for a finite population with $n$ individuals. Let $N$ be a matrix, where each row $N_{i}$ contains one discrete distribution. The matrix will yield $\left(\begin{array}{c}n+k-1 \\ n\end{array}\right)$ rows. Each distribution over information levels can be simulated with the market model, returning a vector of average expected relative market revenues $u\left(N_{i}\right)$. Let $U$ be a matrix which captures the revenues corresponding to the rows in $N$, i.e., $U_{i}=u\left(N_{i}\right)$. A heuristic payoff table $H=(N, U)$ is proposed in Walsh et al. (2002) to capture the payoff information for all possible discrete distributions in a finite population.

In order to approximate the payoff for an arbitrary mix of strategies $x$ in an infinite population distributed over the phenotypes according to $x, n$ individuals are drawn randomly from the infinite distribution. The probability for selecting a specific row $N_{i}$ can be computed from $x$ and $N_{i}$ :

$$
P\left(N_{i} \mid x\right)=\left(\begin{array}{c}
n \\
N_{i, 1}, N_{i, 2}, \ldots, N_{i, k}
\end{array}\right) \prod_{j=1}^{k} x_{j}^{N_{i, j}}
$$

The expected payoff $f_{i}(x)$ is computed as the weighted combination of the payoffs given in all rows, compensating for payoff that cannot be measured. If a discrete distribution features zero traders of a certain information type, its payoffs cannot be measured and $U_{j, i}=0$.

$$
f_{i}(x)=\frac{\sum_{j} P\left(N_{j} \mid x\right) U_{j, i}}{1-\left(1-x_{i}\right)^{k}}
$$

This expected payoff can be used in (3.2) to compute the evolutionary change according to the replicator dynamics. 

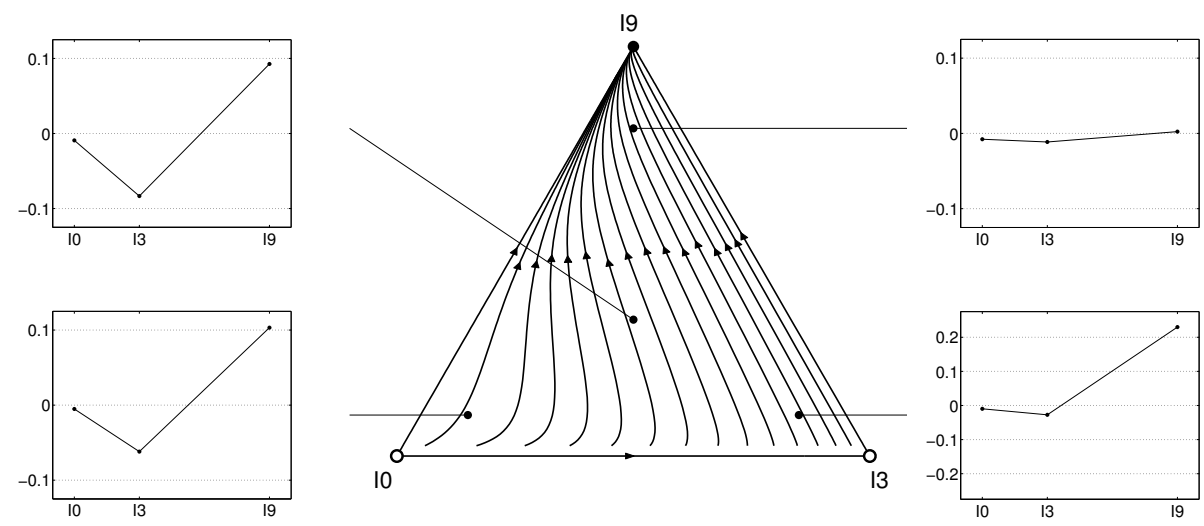

Figure 6.5: The central simplex shows the evolutionary dynamics of an infinite population mixing between the information levels 0,3 and 9 . Relative market revenue over information levels is given for four selected finite distributions: top-left $(33,33,33)$ which reflects a uniform distribution, bottom-left (80, IO, Io), top-right (Iо, Iо, 80), bottomright (Iо, 80, Iо).

\section{Experimental setup and results}

The experiments of this section comprise two elements. An evolutionary analysis of an infinite population is performed to elicit the dependence of revenue on the presence of other information levels. In addition, selected population distributions are approximated with a finite population and illustrate revenue distributions for interesting points. The evolutionary analysis is based on the market model described in Section 6.2 and uses the method described in the previous section to compute payoffs and the replicator dynamics for an infinite population of traders with arbitrary and evolving information distributions. The heuristic payoff tables are computed for $n=12$ traders distributed over the information levels $I 0, I 3$ and $I 9$, leading to 91 rows.

Figure 6.5 shows the evolutionary dynamics of the market model. Four representative population distributions are evaluated in more detail in a finite population of $n=100$ traders to illustrate the revenue structure for the information levels. The evolutionarily stable state is a global attractor, where only insiders prevail. The relative market performance for the four selected finite distributions of traders highlights again the J-curve observed before. Even though non-informed traders perform close to market average, insiders take advantage of their knowledge and take over the market. However, their competitive advantage is vanishing as they are facing more and more competitors of the same information level (see top-right revenue graph of Figure 6.5).

Note that up till now, information was freely available to all traders. However, it 

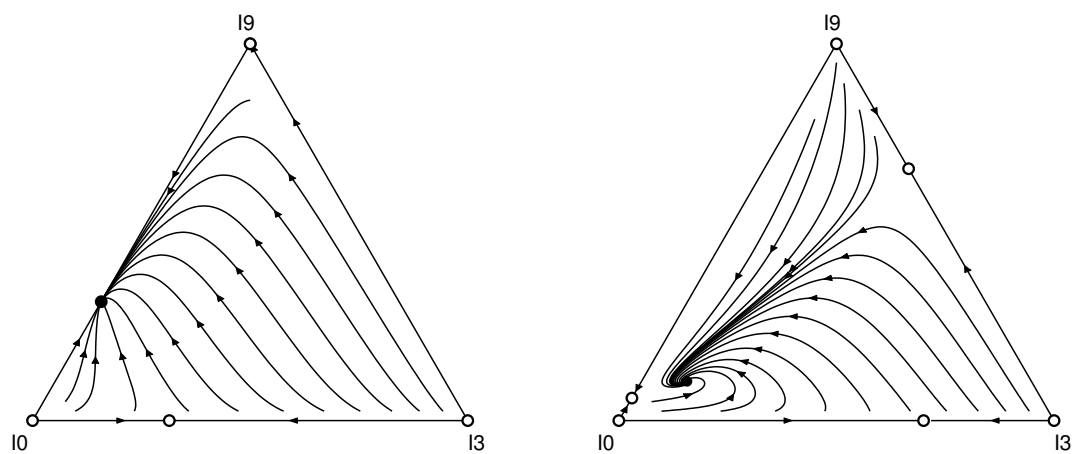

Figure 6.6: Evolutionary market dynamics using a fixed cost (left) and quadratic cost function (right) for information levels 0,3 and 9 .

is reasonable to assume that gathering more information is costly. In the most simple case, a fixed cost for information might be incurred leading to a possible advantage of non-informed traders as they do not have to pay this price. More realistically, costs could also increase with the amount of information gathered, for example using a quadratic cost function such that average-informed traders pay only a little whereas insiders pay the full price. This relates to a real-world scenario where average traders only subscribe to financial newspapers or magazines, whereas insiders may need to hire experts to gain advantage.

Figure 6.6 shows the market dynamics in both cost scenarios. The fixed cost is set to 5 units cash per trading period for information levels $I 3$ and $I 9$, non-informed traders pay nothing. The quadratic cost function used is

$$
\frac{i^{2}}{9^{2}} \cdot 15
$$

where $i$ is the information level, resulting in a maximum cost of 15 units cash for insiders per trading period. As can be observed, introducing cost leads to significantly different and more complex dynamics. In the constant cost scenario, the evolutionary advantage of insiders decreases in favor of non-informed traders, leading to an equilibrium state where insiders and non-informed traders co-exist. Using a quadratic cost function gives rise to an interior equilibrium, mixing between all information levels.

\section{Discussion}

Information does come at a cost in real markets, which has been neglected in much of the related work (Huber, 2007; Huber et al., 2008; Kirchler, 2010; Tóth and Scalas, 
2007; Tóth et al., 2006, 2007). Evolutionary analysis under different cost functions indicates that costs can significantly alter the market dynamics and allow less-informed traders to prevail.

Our results contribute to the ongoing debate about the strong-form efficient-market hypothesis, which has a large following and growing number of critics (Fox, 20II). It states that prices in financial markets instantly reflect all information available to participating traders, including insider information. We found that evolutionary pressure drives a market toward an information distribution at which the market is strongform efficient, possibly driving some information levels extinct in the process. However, the evolutionary process will only end in equilibrium for an isolated system; in real markets, traders that enter the market with information and money from other sources continuously perturb the system. As a result, real markets may be found offequilibrium almost all the time. It is up to future experiments to quantify the influence of arriving traders on perturbation from the equilibrium.

\subsection{Summary}

Literature has established a link between human traders and a market model that can be rigorously analyzed in simulation. In this chapter, we have exploited this link and contributed in the following ways: (I) The value of information in markets has been confirmed to follow a J-curve for several more realistic information distributions. (2) The evolutionary advantage of information makes insiders drive less-informed traders out of the market, with a diminishing competitive edge. (3) If information comes at a cost, less-informed traders may prevail in the market. 



\section{7 \\ Collision Avoidance}

Collision avoidance is relevant for a variety of domains and applications, e.g., crowd dynamics as well as autonomous or assisted control of automobile, aircraft, vessel, or multi-robot systems. Each of the above is of high importance in everyday-life, and of great theoretical and practical interest. Crowd dynamics (Helbing and Johansson, 2009) is the study of pedestrian motion and how individuals affect each others' movements locally. A better understanding of crowd dynamics makes it possible to accurately simulate emergency and evacuation scenarios, resulting in improved guidelines to prevent blockages and jamming in case of a panic stampede.

Driver assistance or fully autonomous cars are further examples of applications requiring collision avoidance systems. Aircraft and vessel traffic follows predefined sets of rules to avoid collisions or resolve such situations. Automobile, aircraft and vessel traffic are application areas of collision avoidance where a game-theoretic analysis has been applied (Lachner et al., 2000; Shi et al., 2007; Xiaohui et al., 2012).

Finally, with the expected increase in the number of robots deployed in factories as well as in home environments, there is a need for methods to avoid collision situations in a variety of multi-robot systems (Rebollo et al., 2007; van den Berg et al., 2008).

In this chapter we analyze the evolutionary stability of various local collision avoidance methods in simulation. Local collision avoidance is the task of steering free of collisions with static and dynamic obstacles, while following a global plan to navigate towards a goal location. Static obstacles can be avoided using traditional planning algorithms whereas dynamic obstacles pose a tough challenge. An intuitive approach is to observe consecutive obstacle positions in order to extrapolate the future trajectory. The velocity obstacle (Fiorini and Shiller, I998) is a geometric representation of all velocities that will eventually result in a collision given that the dynamic obstacle maintains the observed velocity. Velocity obstacles find application in robotics (van den Berg et al., 2008; Snape et al., 20Ir; Hennes et al., 20I2b; Claes et al., 20I2a,b) and have also been applied to the study of crowd dynamics (Guy et al., 20I2). 
Table 7.I: Payoff table of the game of chicken.

\begin{tabular}{c|c|c|}
\multicolumn{1}{c}{} & \multicolumn{1}{c}{ Swerve } & \multicolumn{1}{c}{ Straight } \\
\cline { 2 - 3 } Swerve & 0,0 & $-1,1$ \\
\cline { 2 - 3 } Straight & $1,-1$ & $-10,-10$ \\
\cline { 2 - 3 } & &
\end{tabular}

\section{I The Game of Chicken}

We first consider a stylized example that captures the situation of collision avoidance: the game of chicken (Rapoport and Chammah, I966).

Example 5 (The game of chicken). Two drivers are headed at high speeds for a narrow passage from opposite directions. If both drivers continue to drive straight the result is a catastrophic head-on collision. Whoever swerves is considered a "chicken" and yields the way to the other driver. The best outcome for a driver is thus continuing straight while the other swerves and becomes the "chicken" thereby successfully avoiding a collision.

We assume identical driver reaction times and turning radii of the cars. Therefore, this strategic situation occurs in the last instant before a crash is unavoidable. Each driver faces the choice of continuing straight or swerving to the side. The decisions are taken simultaneously and can not be revoked. The payoffs for the game of chicken are shown in Table 7.I. There are two pure equilibria in the game of chicken, "straightswerve" with payoffs $1,-1$ and "swerve-straight" with payoffs: $-1,1$. Neither player has a dominant strategy as each player's best strategy depends on the strategy played by the adversarial. In addition, there is one symmetric mixed Nash equilibrium where both players play "straight" with probability $\frac{9}{10}$ and "swerve" with probability $\frac{1}{10}$.

A variant of the game of chicken, called the "hawk-dove" game, is studied in the early evolutionary game theoretic literature (Maynard Smith, I974). The strategy "hawk" represents an aggressive player and the strategy "dove" a peaceful contestant. Players stand in competition for a resource. If two aggressive players meet the value of the resource is shared as is the cost of the fight (the cost of fighting is usually assumed to be much higher than the value of the resource). If a "hawk" meets a "dove", the aggressive player receives the full value of the resource, while the other player goes away emptyhanded. Two peaceful players share the resource without the fight. In our example of the game of chicken, the aggressive "hawk" strategy is driving straight. The resource is the narrow road passage and the cost of fighting is the cost of an impact.

Figure 7.I shows the symmetric continuous-time replicator dynamics in the game of chicken with payoff values as shown in Table 7.I. All interior points converge to the mixed Nash equilibrium, which is an evolutionarily stable strategy. The symmet- 


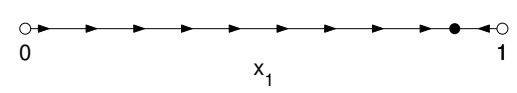

Figure 7.I: Symmetric replicator dynamics of the game of chicken. State $x_{1}=0$ corresponds to a population of "swerve-only", while $x_{1}=1$ corresponds to a population of "straight-only". State $x=\left(\frac{9}{10}, \frac{1}{10}\right)$ is the unique asymptotically stable fixed point of the dynamics.

ric continuous-time replicator dynamics model a population of individuals that are matched up at random and play a one-shot game, see Chapter 3. The dynamics shown in Figure 7.I are thus the result of the same collision avoidance interaction occurring over and over with different random drivers.

\subsection{Velocity Obstacles}

Clearly, the game of chicken presents a very abstract (and radical) situation. Collision avoidance in real domains requires more elaborate decision making than simply choosing between driving straight or swerving to the side. One approach to collision avoidance in continuous spaces is the velocity obstacle paradigm. The velocity obstacle (VO) was first introduced by Fiorini and Shiller (I998) for local collision avoidance and navigation in dynamic environments with multiple moving objects. The subsequent definition of the VO assumes planar motions, though the concept extends to three dimensional motions in a straightforward manner.

Let us assume a workspace configuration with two agents on a collision course as shown in Figure 7.2(a). If the position and speed of the moving object (agent $R_{B}$ ) is known to $R_{A}$, we can mark a region in the agent's velocity space that leads to collision under current velocities and is thus unsafe. This region resembles a cone with the apex at $R_{B}$ 's velocity $v_{B}$, and two rays that are tangential to the convex hull of the Minkowski sum of the footprints of the two agents. The Minkowski sum for two sets of points $A$ and $B$ is defined as:

$$
A \oplus B=\{a+b \mid a \in A, b \in B\}
$$

We define the $\oplus$ operator to denote the convex hull of the Minkowski sum such that $A \oplus B$ results in the points on the convex hull of the Minkowski sum of $A$ and $B$. In the example, the two agents have circular footprints with radii $r_{A}$ and $r_{B}$ respectively. The direction of the left and right ray is then defined as:

$$
\begin{aligned}
& \theta_{\text {left }}=\max _{p_{i} \in \mathscr{F}_{A} \oplus \mathscr{F}_{B}} \operatorname{atan} 2\left(\left(p_{\text {rel }}+p_{i}\right)^{\perp} \cdot p_{\text {rel }},\left(p_{\text {rel }}+p_{i}\right) \cdot p_{\text {rel }}\right) \\
& \theta_{\text {right }}=\min _{p_{i} \in \mathscr{F}_{A} \oplus \mathscr{F}_{B}} \operatorname{atan} 2\left(\left(p_{\text {rel }}+p_{i}\right)^{\perp} \cdot p_{\text {rel }},\left(p_{\text {rel }}+p_{i}\right) \cdot p_{\text {rel }}\right)
\end{aligned}
$$




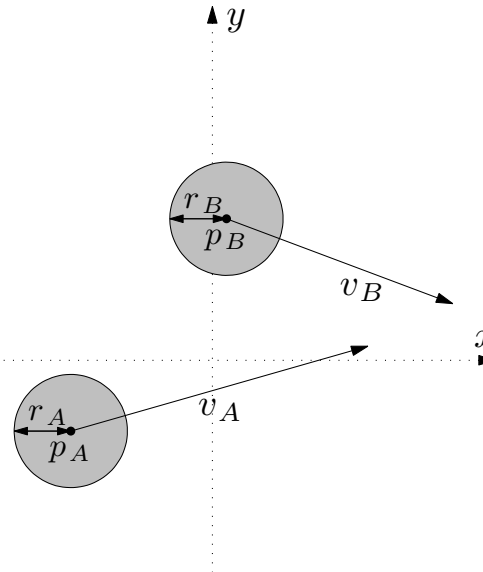

(a) Workspace configuration

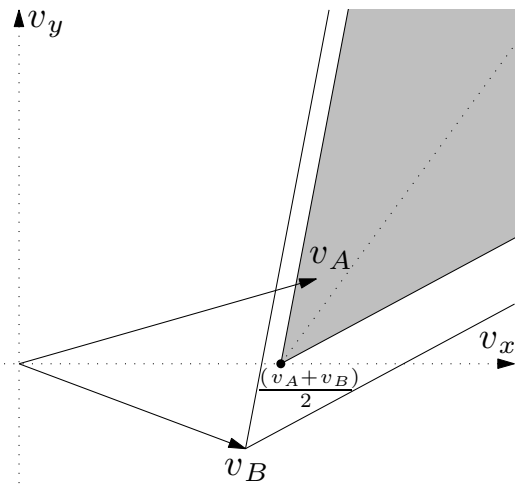

(c) RVO

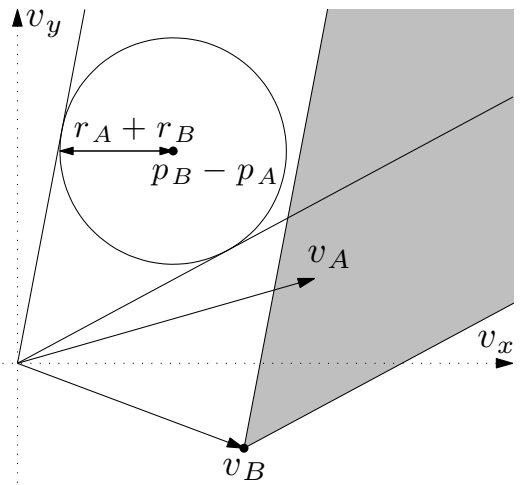

(b) $\mathrm{VO}$

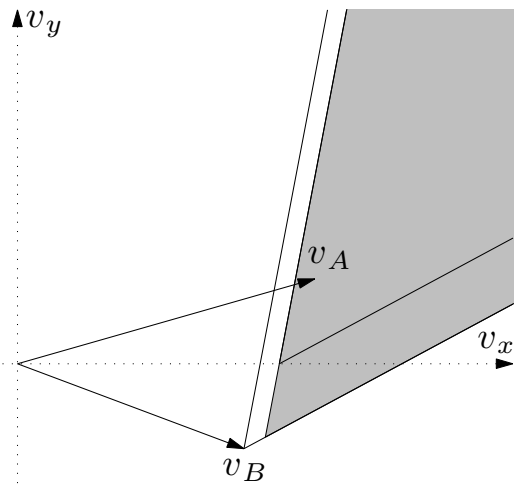

(d) HRVO

Figure 7.2: Creating the different velocity obstacles out of a workspace configuration. (a) A workspace configuration with two agents $R_{A}$ and $R_{B}$. (b) Translating the situation into the velocity space and the resulting velocity obstacle (VO) for $R_{A}$. (c) Translating the VO by $\frac{v_{A}+v_{B}}{2}$ results in the reciprocal velocity obstacle (RVO), i.e., each agent has to take care of half of the collision avoidance. (d) Translating the apex of the RVO to the intersection of the closest leg of the RVO to the own velocity, and the leg of the VO that corresponds to the leg that is furthest away from the own velocity. This encourages passing the agent on a preferred side, i.e., in this example passing on the left. The resulting cone is the hybrid velocity obstacle (HRVO). 
where $p_{\text {rel }}=p_{B}-p_{A}$ is the relative position of the two agents and $\mathscr{F}_{A} \oplus \mathscr{F}_{B}$ is the convex hull of the Minkowski sum of the footprints of the two agents. The atan2 expression computes the signed angle between two vectors. The resulting angles $\theta_{\text {left }}$ and $\theta_{\text {right }}$ are left and right of $p_{\text {rel }}$.

In the example in Figure 7.2, agent $R_{A}$ 's velocity vector $v_{A}$ points into the VO, thus we know that $R_{A}$ and $R_{B}$ are on collision course. Each agent computes a VO for each of the other agents. If all agents at any given time step select velocities outside of the VOs, the trajectories are guaranteed to be collision free. However, oscillations can still occur when the agents are on collision course. Since all agents select a new velocity outside of all velocity obstacles independently, at the next time step, the old velocities pointing towards the goal will become available again. Hence, all agents select their old velocities, which will be on collision course again after the next time step.

To overcome these oscillations, the reciprocal velocity obstacle (RVO) was introduced by van den Berg et al. (2008). The surrounding moving obstacles are in fact also pro-active agents and thus aim to avoid collisions too. Assuming that each agent takes care of half of the collision avoidance, the apex of the VO can be translated to $\frac{v_{A}+v_{B}}{2}$. Furthermore, this leads to the property that if every agent chooses a velocity outside of the RVO closest to the current velocity, the agents will pass on the same side. However, each agent optimizes its commanded velocity with respect to a preferred velocity in order to make progress towards its goal location. This can lead to reciprocal dances, i.e., where both agents first try to avoid to the same side and then to the other side. In a situation with perfect symmetry and sensing, this behavior continues indefinitely.

To counter these situations, the hybrid velocity obstacle (HRVO) was introduced by Snape et al. (2OII). Figure 7.2(d) shows the construction of the HRVO. To encourage the selection of a velocity towards the preferred side, e.g., left in this example, the other leg of the RVO is substituted with the corresponding leg of the VO. The new apex is the intersection of the line of the one leg from RVO and the line of the other leg from the VO. This reduces the chance of selecting a velocity on the "wrong" side of the velocity obstacle and thus the chance of a reciprocal dance, while not overconstraining the velocity space. The agent might still try to pass on the "wrong" side, e.g., another agent induces a HRVO that blocks the whole side, but then soon all other agents will adapt to the new side too.

\section{Truncation}

When the workspace is cluttered with many agents that do not move or only move slowly, the apices of the HRVOs are close to the origin in velocity space; thus rendering agents immobile. This problem can be solved using truncation. The idea of a truncated hybrid velocity obstacle can be best explained by imagining a static obstacle. 


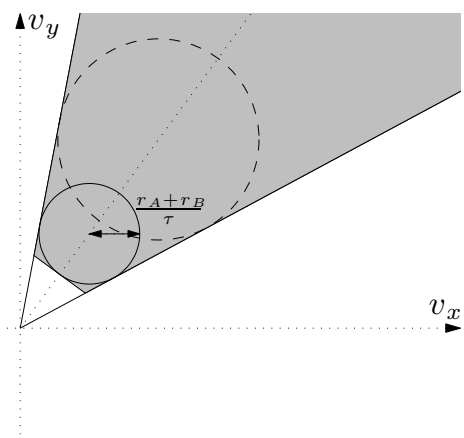

(a) Truncated VO

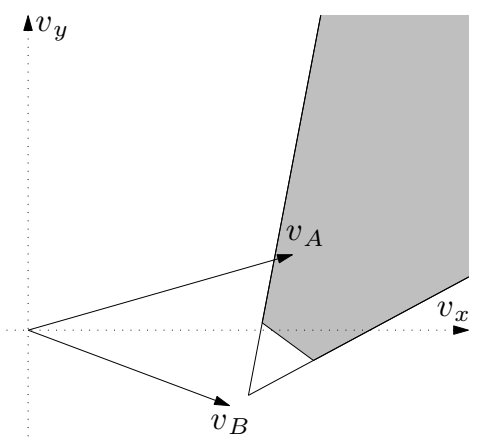

(b) Truncated HRVO

Figure 7.3: (a) Truncation of a $\mathrm{VO}$ of a static obstacle at $\tau=2$ and approximating the truncation by a line. (b) Translating the truncated cone according to the HRVO method to get a truncated HRVO.

A velocity in the direction of the obstacle will eventually lead into collision, but not directly. Hence, we can define an area in which the selected velocities are safe for at least $\tau$ time steps. The truncation is then in the shape of the Minkowski sum of the two footprints, shrunk by the factor $\tau$. If the footprints are discs, the shrunken disc that still fits in the truncated cone has a radius of $\frac{r_{A}+r_{B}}{\tau}$, see Figure 7.3(a). The truncation can be closely approximated by a line perpendicular to the relative position and tangential to the shrunken disk. Applying the same method to create a HRVO from a $\mathrm{VO}$, we can create a truncated HRVO out of the truncated VO by translating the apex accordingly, see Figure 7.3(b). The same applies to RVOs.

\section{ClearPath}

To efficiently compute collision free velocities, we employ the ClearPath algorithm introduced by Guy et al. (2009). The algorithm is applicable to many variations of velocity obstacles (VO, RVO or HRVO) represented by line segments or rays. ClearPath follows the general idea that the collision free velocity that is closest to preferred velocity is: (a) on the intersection of two line segments of any two velocity obstacles, or (b) the projection of the preferred velocity onto the closest leg of each velocity obstacle. All points that are within another obstacle are discarded and from the remaining set the one closest to the preferred velocity is selected. Figure 7.4 shows the graphical interpretation of the algorithm.

\subsection{Evolutionary Analysis}

We have introduced three variations of the velocity obstacle approach, namely VO, RVO, and HRVO. Evaluating the performance of these methods, especially in a het- 


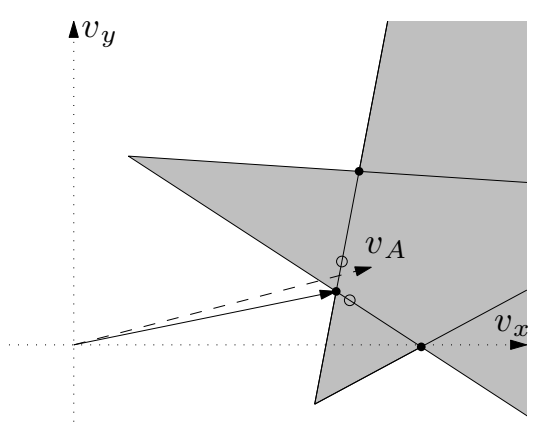

Figure 7.4: ClearPath enumerates intersection points for all pairs of VOs (solid dots). In addition the preferred velocity $v_{A}$ is projected on the closest leg of each $\mathrm{VO}$ (open dots). The point closest to the preferred velocity (dashed line) and outside of all VOs is selected as new velocity (solid line).

erogenous setting, is the aim of this chapter. We perform an evolutionary analysis based on heuristic payoff tables (Walsh et al., 2002) to approximate an infinite population. The heuristic payoff table $H$ captures the payoff information for all possible discrete distributions $N_{i}$ for a finite population with $n$ individuals. The payoff for an arbitrary continuous population state $x$ is computed as the weighted average over all rows of the heuristic payoff table, where payoffs are weighted by the probability that the discrete distribution of a particular row $N_{i}$ is the result of drawing $n$ individuals according to $x$. For further details, see Section 6.3.

\section{Experimental Setup}

To compute the payoffs corresponding to each finite population $N_{i}$ we consider the following scenario. All agents have a circular footprint with a radius of $0.2 \mathrm{~m}$ and move with a maximum speed of $0.5 \mathrm{~m} / \mathrm{s}$. Agents are initially located on a circle (equally spaced) with a radius of $10 \mathrm{~m}$ and the goal locations are set to the antipodal positions, i.e., each agent's shortest path is through the center of the circle. Figure 7.5 shows example trajectories for 6 agents. The goal is assumed to be reached when the agent's center is within a $0.01 \mathrm{~m}$ radius of the goal location. The performance of agent $i$ is the negative value of its time of arrival, denoted as $-T_{i}$. Heuristic payoff tables are computed for $n=12$ agents, leading to 91 rows. Payoffs for each discrete distribution $N_{i}$ are averaged over (a maximum of) 20 random permutations on the initial positions of agents. ${ }^{\mathrm{I}}$

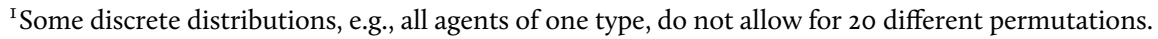




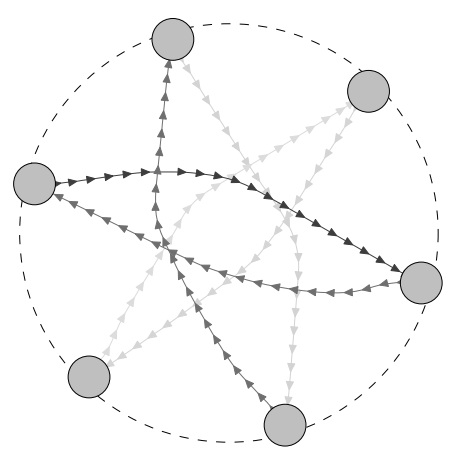

Figure 7.5: Trajectories of 6 RVO agents initially positioned on a circle with goal locations set to the antipodal positions.

\section{Results and Discussion}

Figure 7.6 shows the evolutionary dynamics of a population with agents of types VO, $\mathrm{RVO}$, and HRVO using no truncation of the velocity obstacles, i.e., $\tau=\infty$. All "pure" population states are asymptotically stable fixed points under the replicator dynamics. However, the strategy space is not partitioned equally between all attractors, the basin of attraction for RVO is considerably smaller. Between each pair of strategies, there is one repeller at the uniform mixture. In addition, there is one saddle point at $(0.29,0.49,0.22)$.

We do not see any dominant strategy for a heterogeneous setting including all three variations of the velocity obstacle. Also for pairwise comparison between two strategies, along the faces of the simplex, no strategy is inferior. All three strategies are evolutionarily stable.

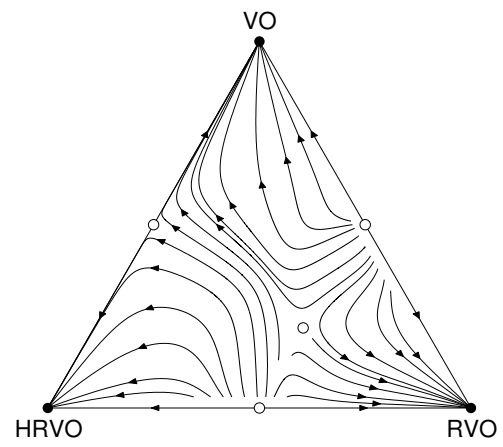

Figure 7.6: Evolutionary dynamics of a population mixing between the avoidance strategies: velocity obstacle (VO), reciprocal velocity obstacle (RVO) and hybrid-velocity obstacle (HRVO). Asymptotically stable attractors are depicted by solid circles; unstable rest points are shown as open circles. 


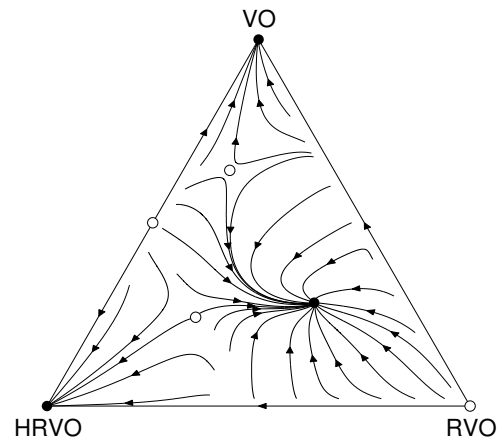

(a) Truncated VO, RVO and HRVO with $\tau=10$.

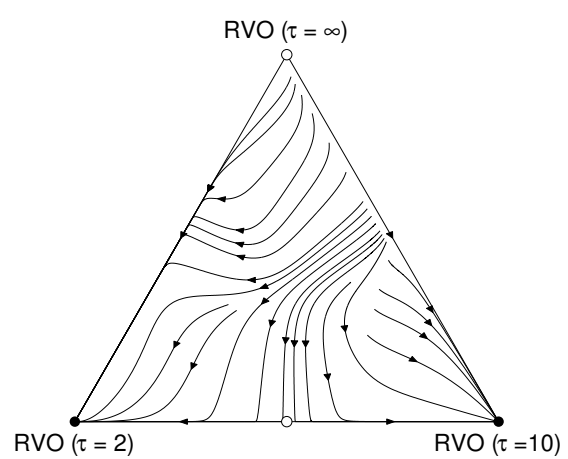

(b) Truncated RVO.

Figure 7.7: Evolutionary dynamics of collision avoidance with truncation. Asymptotically stable attractors are depicted by solid circles; unstable rest points are shown as open circles.

Figure 7.7(a) shows the dynamics for the same strategies with truncation $\tau=10$. Strategies VO and HRVO are still asymptotically stable, while RVO is a repeller. The interior stable fixed point at $(0.23,0.49,0.28)$ has the largest basin of attraction amounting to more than half of the strategy space.

Introducing truncation leads to significantly different and more complex dynamics. In a pairwise comparison (faces of the simplex), RVO is dominated by VO as well as HRVO. However, the reciprocal velocity obstacle is most robust in the presence of all three strategies (interior of the simplex). Considering Figure 7.2, we see that RVO is the most "aggressive" or least restricting velocity obstacle. The collision space of a RVO is always a subset of the corresponding VO for moving obstacles. This is due to the assumption that other agents take care of half of the collision avoidance. VO and HRVO are both more conservative and thus restrict the admissible velocity space more.

Finally, Figure 7.7(b) shows a comparison of different levels of truncation for the reciprocal velocity obstacle. In particular, we use $\tau=\infty$ (no truncation), $\tau=2$, and $\tau=10$. In this comparison agents using RVOs with no truncation $(\tau=\infty)$ are strictly dominated; both "pure" population states using truncation are asymptotically stable. Truncation with $\tau=2$ has the largest basin of attraction.

Truncation with low values for $\tau$ is less restrictive and agents continue on a straight path until the truncated velocity obstacle takes affect. As such, the average time of arrival is shorter and the performance increases. In the presence of agents employing velocity obstacles with less truncation, distinct use of truncation leads to situations where agents are "trapped" near the center. In particular, agents with $\tau=2$ drive straight towards the center, while agents with $\tau=10$ see affect of the velocity obstacles sooner and enter in a spiralling motion. 


\subsection{Summary}

We have studied three variations of the velocity obstacle approach in competition, i.e., the velocity obstacle, the reciprocal velocity obstacle and the hybrid velocity obstacle. Without truncation all three types perform equally well and we do not find a dominant strategy. With the use of truncated velocity obstacles the dynamics become more complex; the reciprocal velocity obstacle is most robust in the heterogenous system, however, in pairwise comparison this strategy is inferior. 


\section{8 \\ Conclusion}

In this final chapter, we summarize and discuss the main contributions of this thesis. In particular, we present answers to the questions that were set forward in Chapter I and guided this research. Finally, we discuss limitations and illustrate several perspectives for future research.

\section{I Contributions and Answers to the Research Questions}

In Section I.3 we have put forward six research questions, three questions concerning the theoretical framework of evolutionary models of learning, and three questions concerned with the application of this framework to complex real world problems. Each research question is answered and the corresponding contributions are summarized below.

Question I: What is the multiagent learning interpretation of a single population under the symmetric continuous time replicator dynamics?

In Section 3.4 we have derived the dynamics of a large population of learners which are randomly matched to play a symmetric game. This combines two evolutionary interpretations: (I) a large population of individuals randomly engaging in an interaction and (2) the learning analogy of an adaptive agent maintaining a population of possible strategies encoding its policy. If learners are selected uniformly to play the game, the information encoded in the behavior of each learner is important to the learning process even though the system assumes a large number of learners. If fitness proportionate selection is used, we observe that the overall population average moves independently of the behavior of individual learners. We thus conclude that the symmetric continuous time replicator dynamics are a valid model for large multiagent systems of independent reinforcement learners under fitness proportionate selection. 
Question 2: To what extent does the relation between asymmetric continuous time replicator dynamics and individual reinforcement learning generalize to stochastic games?

In Chapter 4 we have provided a formalization of piecewise replicator dynamics, a first approach to model multi-state learning dynamics. In particular, we have extended previous work by providing general definitions for the concepts of average reward games, pure equilibrium cells and piecewise replicators. Consecutively, we have identified shortcomings of piecewise replicator dynamics, i.e., discontinuities and occurrences of qualitative anomalies. State-coupled replicator dynamics were proposed in Section 4.2 to alleviate these disadvantages. The preceding formalization of piecewise replicators was deliberately factored into the new approach. We have delivered a comparative study of networks of finite action-set learning automata as well as piecewise and state-coupled replicator dynamics. State-coupled replicators have been shown to predict learning dynamics in stochastic games more accurately than their predecessor, the piecewise model. Finally, we have derived the symmetric state-coupled replicator dynamics in Section 4.3. For a symmetric two-player stochastic game the state-coupled replicator dynamics select two individuals at random from the same population in each state.

Question 3: What is the stochastic game generalization of the selection-mutation model of Q-learning by Tuyls et al. (2003b)?

We have continued our analysis of the stochastic game generalization by introducing an exploration-mutation term in the state-coupled dynamics in Section 4.4. More precisely, we have linked the stateless dynamics of learning automata to $Q$-learning. We have found that the exploration-mutation term of the $Q$-learning dynamics solely depends on the population that encodes the policy. As such, the average reward game as defined previously remains the sound measure for the fitness computation and the state-coupled replicator dynamics can be extended with the mutation term. Finally, we have shown that state-coupled replicator dynamics with mutation converge in games with mixed optimal strategies and we have provided an independent reinforcement learning algorithm that matches the predicted learning behavior.

Question 4: How can the evolutionary model of learning be applied to complex real-world problems such as multiagent bargaining situations?

In Chapter 5 we have investigated complex bargaining situations modeled in the Colored Trails test-bed. We have specified metastrategies that characterize players' bargaining behavior and allow us to map a complex three player take-it or leave-it bargaining game to a set of bilateral normal-form games that are characterized by well-known 
social dilemmas, i.e., prisoners' dilemma, stag hunt and ultimatum games. The definition of metastrategies has facilitated the theoretical analysis and has also allowed us to apply the methodology of evolutionary game theory and thus evolutionary models of learning to this complex domain. We have provided an empirical analysis of a large set of game instances and have analyzed the evolutionary dynamics under different assumptions on rationality and social factors. Under the assumption of a purely rational responder, results show a mixed equilibrium between the two strategies that favor either the proposer or the responder. For games with inequity averse responders, the basin of the responder focused attractor increases. In general, we observe that a proposal situated on the Pareto front balancing the interests of the proposer and the responder performs best and constitutes the largest basin of attraction in the corresponding population dynamics.

Question 5: What is the value of future price signal information; and in particular: is there an evolutionary advantage of foresight in markets?

In Chapter 6 we have studied the competitive advantage of price signal information for traders in a simulated continuous double auction. Previous work has established that more information about the price development does not guarantee higher performance. In particular, traders with limited information perform below market average and are outperformed by random traders; only insiders beat the market. However, this result has only been shown in markets with few traders and a uniform distribution over information levels. We have presented additional simulations of several more realistic information distributions that confirm previous findings. In addition, we have analyzed the market dynamics with an evolutionary model of competing information levels. Results have shown that the highest information level will dominate if information comes for free. If information is costly, less-informed traders may prevail reflecting a more realistic distribution over information levels.

Question 6: What are the evolutionary dynamics of collision avoidance; and in particular, is reciprocity in collision avoidance an evolutionarily stable strategy?

In Chapter 7 we have studied three variations of the velocity obstacle approach in competition, i.e., the velocity obstacle, the reciprocal velocity obstacle and the hybrid velocity obstacle. Velocity obstacles offer a geometrical representation of the velocity space that results in a collision if all agents maintain current velocities. The reciprocal velocity obstacle assumes that other agents also react to prevent collisions and takes this into account. The hybrid velocity obstacle is a combination of the former two methods. Results show that without truncation all three obstacle types perform equally well and we have not found a dominant strategy. With the use of truncated velocity obstacles 
the dynamics become more complex. The reciprocal velocity obstacle is most robust in the heterogenous system, however, in pairwise comparison this strategy is inferior to both the velocity obstacle and hybrid reciprocal velocity obstacle.

Overall, this dissertation addressed the central problem statement and contributed to a better understanding of multiagent learning dynamics as follows: Question I through 3 investigated the replicator dynamics as a formal framework to study the dynamics of reinforcement learning in multiagent systems and in particular extended the framework to include multi-state games. Question 4 through 6 applied this framework to complex real world problems, ranging from applications in multiagent bargaining and market analysis to collision avoidance.

\subsection{Limitations and Future Research Perspectives}

The aim of this dissertation was to use the replicator dynamics as a tool and formal framework to study the dynamics of reinforcement learning in multiagent systems. The two main limitations of this approach were (a) its restriction to stateless repeated two-player games and (b) limited application to complex real world problems. We addressed both problems in Part I and Part II of this thesis and our research lead to a number of promising contributions. However, learning in multiagent systems in general, and in particular evolutionary models of such systems, remains a very interesting yet challenging research domain. We list a number of possible extensions to the presented work and perspectives for future research below.

Chapter 3: We have seen that under fitness proportionate selection the overall population average of a population of learners moves independently of the behavior of individual learners. Some domains, such as markets, show the property of fitness proportionate selection as traders that perform better have access to more assets and can thus trade higher volumes (or at a higher frequency). However, fitness proportionate selection is not a given in any large multiagent system. Future research should investigate the possibilities to transfer the idea of fitness proportionate selection to such systems in which selection for bilateral iterations is driven by a deterministic or a non-proportional random process, e.g., uniform selection. This could be implemented through adaptive learning rates similar to the ideas of "Win or Learn Fast" or leniency, see Section 2.6 and 3.3.

Chapter 4: The state-coupled replicator equations in Chapter 4 use the limiting average expected reward criterion. Many value iteration based algorithms such as $Q$ learning use discounted rewards. Deriving the corresponding state-coupled dynamics for the $\beta$-discounted expected reward criterion is an open research question. The 
games used in Chapter 4 are small two-state examples. Although in principle the definition of state-coupled replicator dynamics is general in the number of players and states, the visual approach to analyze basins of attractions remains limited to at most three dimensions or a suitable projection. An interesting future direction of this line of research is thus exploring the connection to finite-element analysis (Hughes, 2000), both for efficient computation and the representation of spacial problems. A third direction is the extension to continuous state- or action-spaces. Although there has been some work on extending the replicator dynamics to continuous action spaces, e.g. by Tuyls and Westra (2009), in particular the link to continuous reinforcement learning using function approximation remains to be investigated. The combination of both continuous action- and state-spaces relates naturally to the framework of differential game theory rather than stochastic games. Differential games (Isaacs, I999) model the iteration between players as a system of differential equations, often equations of motion. Integrating both the evolutionary model, itself a system of differential equations, and a dynamical representation of the game through differential game theory, is a very interesting future research direction.

Chapter 5: Colored Trails is an interesting test-bed to study human behavior and decision making under bounded rationality. The metastrategies as identified in Chapter 5 are the result of a game theoretic analysis under different assumptions on the behavior of the responder. Studies in the area of behavioral economics or behavioral game theory are necessary in order to determine if human players indeed play according to these metastrategies. Two of the many questions that arise are: Do humans play strategies that lie on the convex hull of the gain graph? And if so, does the learning process over repeated interactions lead to the dynamics as predicted by the evolutionary analysis? The predictive quality of evolutionary game theory on human learning in strategic interactions has been studied in small matrix games (Erev and Roth, 1998; Hopkins, 2002) but if these results translate to more complex situations is unclear.

Chapter 6: In Chapter 6 we have studied the market dynamics of fundamental traders with varying amounts of information on future prices. However, there are two main types of trading strategies in todays markets: fundamentalists and chartists (Taylor and Allen, I992; Gehrig and Menkhoff, 2006). Fundamentalists use a forecasting model that fits the actual economy and correctly identify the fundamental driving forces of the market. Technical analysts, also called chartists, use an autoregressive process to predict future price developments based on recent trends. One might be tempted to conjecture that fundamentalists eventually drive chartists out of the market. After all, chartists try to exploit an autocorrelation structure in the price series, which in turn is mainly a result of their own trading behavior - not an underlying feature of the market. Rational fundamentalists must surely be superior as they base trading decisions 
on actual fundamental facts. However, fundamentalists are not strictly rational. Future fundamental values (e.g. earnings or dividends) of a company are not known at present time and must be predicted using a model. The model must match the economy that drives the market and model parameters must be adjusted accordingly. A mismatch in model choice, or uncertainty in parameter estimates that deviate from the ones that determine the underlying process, inevitably cause bounded rationality and thus the risk for false decisions. An intriguing and promising area for future research is thus to analyze the market dynamics in the presence of fundamentalists, chartists and zero information traders. In particular, an interesting research question is to what extend chartists can survive in a market and coexist with fundamentalists or if indeed chartists are eventually driven out of the market.

Chapter 7: The evaluation in Chapter 7 is based on a scenario commonly used in literature to showcase the velocity obstacle approach, i.e., agents are initially located on a circle with their goal locations set to the antipodal positions (van den Berg et al., 2008; Snape et al., 20II; Hennes et al., 20I2b; Claes et al., 20I2a). A natural extension is to consider various other scenarios, e.g., agents moving in a free space or in the presence of obstacles with randomly generated goal locations. However, it must be taken into account that such a setting requires a global navigation strategy, which might have an effect on the performance of the local collision avoidance. Furthermore, the extension to a less symmetric and stylized configuration also allows to evaluate aggressive "straight" driving agents that do not adhere to any of the collision avoidance obstacles as suggested in the game of chicken. 


\section{References}

Abdallah, S. and Lesser, V. (2008). A Multiagent Reinforcement Learning Algorithm with Non-linear Dynamics. Journal of Artificial Intelligence Research, 33:52 I-549.

Akchurina, N. (2009). Multiagent reinforcement learning: algorithm converging to Nash equilibrium in general-sum discounted stochastic games. In Proceedings of the Eighth International Joint Conference on Autonomous Agents and Multiagent Systems, volume 2, pages $725-732$.

Angel, J. J. (2002). Market mechanics: A guide to U.S. stock markets. Technical report, The Nasdaq Stock Market Educational Foundation, Inc.

Aumann, R. J. (1959). Acceptable points in general cooperative n-person games. In Contributions to the Theory of Games, Volume IV., pages 287-324. Princeton University Press.

Axelrod, R. (1984). The Evolution of Cooperation. Basic Books, New York.

Axelrod, R. and Hamilton, W. (I98I). The evolution of cooperation. Science, 2II:I390I396.

Bajari, P. and Hortacsu, A. (2003). The winner's curse, reserve prices, and endogenous entry: empirical insights from ebay auctions. RAND Journal of Economics, pages $329-355$.

Banerjee, B. and Peng, J. (2003). Adaptive policy gradient in multiagent learning. In Proceedings of the Second International Joint Conference on Autonomous Agents and Multiagent Systems, pages 686-692.

Banerjee, B., Sen, S., and Peng, J. (200I). Fast concurrent reinforcement learners. In Proceedings of the Seventeenth International Joint Conference on Artificial Intelligence, pages 825-832, Seattle, WA. Morgan Kaufmann.

Barrot, C., Albers, S., Skiera, B., and Schäfers, B. (20I0). Vickrey vs. ebay: Why secondprice sealed-bid auctions lead to more realistic price-demand functions. International Journal of Electronic Commerce, I4(4):7-38.

Bellman, R. E. (1957). Dynamic Programming. Princeton Landmarks in Mathematics and Physics, 20Io reprint. Princeton University Press. 
Bloembergen, D., Kaisers, M., and Tuyls, K. (2010a). A comparative study of multiagent reinforcement learning dynamics. In Proc. of 22nd Benelux Conf. on Artificial Intelligence (BNAIC 20IO). University of Luxembourg.

Bloembergen, D., Kaisers, M., and Tuyls, K. (20Iob). Lenient frequency adjusted qlearning. In Proceedings of 22nd Benelux Conf. on Artificial Intelligence (BNAIC 2OIO), pages i9-26. University of Luxembourg.

Börgers, T. and Sarin, R. (I997). Learning through reinforcement and replicator dynamics. Journal of Economic Theory, 77(I).

Bowling, M. (2005). Convergence and no-regret in multiagent learning. In Advances in Neural Information Processing Systems I7, pages 209-216. MIT Press.

Bowling, M. and Veloso, M. (200o). An analysis of stochastic game theory for multiagent reinforcement learning. Technical report, Computer Science Department, Carnegie Mellon University.

Bowling, M. and Veloso, M. (2002). Multiagent learning using a variable learning rate. Artificial Intelligence, 136:215-250.

Bowling, M. H. (2000). Convergence problems of general-sum multiagent reinforcement learning. In Proceedings of the Seventeenth International Conference on Machine Learning, pages 89-94. Morgan Kaufmann Publishers Inc.

Boyd, R. and Richerson, P. J. (1985). Culture and the Evolutionary Process. University of Chicago Press, Chicago.

Brafman, R. I. and Tennenholtz, M. (2002). R-max - a general polynomial time algorithm for near-optimal reinforcement learning. Journal of Machine Learning Research, 3:213-23I.

Brooks, R. A. (I99I). Intelligence without reason. In Proceedings of the International Joint Conference on Artificial Intelligence (IJCAI).

Brown, G. (I95I). Iterative solutions of games by fictitious play. In Koopmans, T. C., editor, Activity Analysis of Production and Allocation, pages 374-376, New York. John Wiley \& Sons, Inc.

Bush, R. R. and Mosteller, F. (I955). Stochastic models for learning. John Wiley \& Sons, Inc.

Buşoniu, L., Babuška, R., and De Schutter, B. (2008). A comprehensive survey of multiagent reinforcement learning. IEEE Transactions on Systems, Man, and Cybernetics, Part C: Applications and Reviews, 38(2):156-I72. 
Buşoniu, L., Babuška, R., De Schutter, B., and Ernst, D. (20I0). Reinforcement Learning and Dynamic Programming Using Function Approximators. CRC Press, Boca Raton, Florida.

Chang, Y.-H., Maheswaran, R., and Levinboim, T. (2010). The social ultimatum game. In NIPS Workshop on Decision Making with Imperfect Decision Makers.

Claes, D., Hennes, D., Meeussen, W., and Tuyls, K. (2012a). CALU: Multi-robot collision avoidance with localization uncertainty (Demonstration). In Proceedings of IIth International Conference on Adaptive Agents and Multi-agent Systems (AAMAS 20I2), Valencia, Spain.

Claes, D., Hennes, D., and Tuyls, K. (20II). Real-world multi-robot collision avoidance with localization uncertainty. In Proceedings of gth European Workshop on Multiagent Systems (EUMAS 2OII), Maastricht, Netherlands.

Claes, D., Hennes, D., Tuyls, K., and Meeussen, W. (20I2b). Collision avoidance under bounded localization uncertainty. In Proceedings of IEEE/RSJ International Conference on Intelligent Robots and Systems (IROS 2OI2), Vilamoura, Portugal.

Claus, C. and Boutilier, C. (I998). The dynamics of reinforcement learning in cooperative multiagent systems. In Proceedings of National Conference on Artificial Intelligence (AAAI'98), pages 746-752.

Conitzer, V. and Sandholm, T. (2003). Awesome: A general multiagent learning algorithm that converges in self-play and learns a best response against stationary opponents. In Proceedings of the Twentieth International Conference on Machine Learning, pages 83-90. AAAI Press.

Conitzer, V. and Sandholm, T. (2007). Awesome: A general multiagent learning algorithm that converges in self-play and learns a best response against stationary opponents. Machine Learnearning, 67(I-2):23-43.

Cowles, A. (1933). Can stock market forecasters forecast? Econometrica, I(3):209-324.

Crandall, J. W. (20I2). Just add pepper: extending learning algorithms for repeated matrix games to repeated markov games. In Proceedings of the IIth International Conference on Autonomous Agents and Multiagent Systems - Volume I, pages 399-406, Richland, SC. International Foundation for Autonomous Agents and Multiagent Systems.

Crandall, J. W. and Goodrich, M. A. (20II). Learning to compete, coordinate, and cooperate in repeated games using reinforcement learning. Machine Learning, 82(3):28I3I4. 
de Jong, H., Page, M., Hernandez, C., and Geiselmann, J. (200I). Qualitative simulation of genetic regulatory networks: Method and application. In Proceedings of the International Joint Conferences on Artificial Intelligence (IJCAI).

de Jong, S., Hennes, D., Tuyls, K., and Gal, Y. (20Ira). Meta-strategies in the colored trails game. In Proceedings of the Ioth International Conference on Autonomous Agents and Multi-Agent Systems (AAMAS 2OII), pages 55I-558, Taipei, Taiwan.

de Jong, S., Hennes, D., Tuyls, K., and Gal, Y. (20IIb). Meta-strategies in the colored trails game. In Proceedings of 23 rd Benelux Conference on Artificial Intelligence (BNAIC 20II), pages 383-384, Gent, Belgium.

de Jong, S., Tuyls, K., and Verbeeck, K. (2008). Fairness in multi-agent systems. Knowledge Engineering Review, 23:153-180.

Erev, I. and Roth, A. E. (1998). Predicting how people play games: Reinforcement learning in experimental games with unique, mixed strategy equilibria. American Economic Review, 88(4):848-8I.

Fehr, E. and Schmidt, K. (I999). A Theory of Fairness, Competition and Cooperation. Quart. J. of Economics, II4:8I7-868.

Feigenbaum, J., Fortnow, L., Pennock, D. M., and Sami, R. (2005). Computation in a distributed information market. Theoretical Computer Science, 343(I-2):II4 - I32.

Filar, J. and Vrieze, K. (I996). Competitive Markov Decision Processes. Springer.

Fiorini, P. and Shiller, Z. (I998). Motion planning in dynamic environments using velocity obstacles. International Journal of Robotics Research, I7:760-772.

Fox, J. (20II). The myth of the rational market: a history of risk, reward, and delusion on Wall Street. Harper Paperbacks.

Fudenberg, D. and Levine, D. (1998). The Theory of Learning in Games. MIT Press series on economic learning and social evolution. MIT Press.

Fudenberg, D. and Levine, D. K. (1999). The Theory of Learning in Games. MIT Press.

Fudenberg, D. and Tirole, J. (I99I). Game Theory. MIT Press.

Gal, Y., Grosz, B., Kraus, S., Pfeffer, A., and Shieber, S. (20I0). Agent decision-making in open-mixed networks. Artificial Intelligence, I74:I460-I480.

Gal, Y. and Pfeffer, A. (2007). Modeling reciprocity in human bilateral negotiation. In National Conference on Artificial Intelligence (AAAI). 
Gehrig, T. and Menkhoff, L. (2006). Extended evidence on the use of technical analysis in foreign exchange. International Journal of Finance \& Economics, II(4):327-338.

Gerding, E. H., Rabinovich, Z., Byde, A., Elkind, E., and Jennings, N. R. (2008). Approximating mixed Nash equilibria using smooth fictitious play in simultaneous auctions. In Proceedings of the International Conference on Autonomous Agents and Multiagent Sytems, pages I577-I580.

Gibbons, R. (1992). A Primer in Game Theory. Pearson Higher Education.

Gintis, H. (200o). Game Theory Evolving. A Problem-Centered Introduction to Modelling Strategic Interaction. Princeton University Press, Princeton.

Gjerstad, S. and Dickhaut, J. (1998). Price formation in double auctions. Games and economic behavior, 22(I):I-29.

Gordon, M. (I962). The Investment, Financing, and Valuation of the Corporation. Irwin Series in Economics. Greenwood Press.

Govindan, S. and Wilson, R. (2008). Refinements of Nash equilibrium. In Durlauf, S. N. and Blume, L. E., editors, The New Palgrave Dictionary of Economics. Palgrave Macmillan, Basingstoke.

Greenwald, A. and Hall, K. (2003). Correlated Q-learning. In Proceedings of the Twentieth International Conference on Machine Learning, pages 242-249.

Grosskopf, B. (2003). Reinforcement and directional learning in the ultimatum game with responder competition. Experimental Economics, 6(2):I4I-I58.

Grosz, B. J., Kraus, S., Talman, S., Stossel, B., and Havlin, M. (2004). The Influence of Social Dependencies on Decision-Making. Initial investigations with a new game. In Proceedings of the Third International Joint Conference on Autonomous Agents and Multiagent Systems (AAMAS).

Güth, W., Schmittberger, R., and Schwarze, B. (I982). An experimental analysis of ultimatum bargaining. Journal of Economic Behavior \& Organization, 3(4):367 - 388.

Guy, S. J., Chhugani, J., Kim, C., Satish, N., Lin, M. C., Manocha, D., and Dubey, P. (2009). Clearpath: Highly parallel collision avoidance for multi-agent simulation. In Symposium on Computer Animation.

Guy, S. J., van den Berg, J., Liu, W., Lau, R., Lin, M. C., and Manocha, D. (2012). A statistical similarity measure for aggregate crowd dynamics. ACM Transactions on Graphics, 3I(6):I90:I-I90:II. 
Haim, G., Gal, Y., Kraus, S., and Blumberg, Y. (20I0). Learning Human Negotiation Behavior Across Cultures. In Proceedings of the Second International Working Conference on Human Factors and Computational Models in Negotiation.

Hauert, C. (20I0). Ultimatum game with proposer competition. GameLab experiments.

Helbing, D. and Johansson, A. (2009). Pedestrian, crowd and evacuation dynamics. In Meyers, R. A., editor, Encyclopedia of Complexity and Systems Science, pages 64766495. Springer.

Hennes, D., Bloembergen, D., Kaisers, M., Tuyls, K., and Parsons, S. (20I2a). Evolutionary advantage of foresight in markets. In Proceedings of the Genetic and Evolutionary Computation Conference (GECCO 20I2), pages 943-949, Philadelphia, United States.

Hennes, D., Claes, D., Meeussen, W., and Tuyls, K. (20I2b). Multi-robot collision avoidance with localization uncertainty. In Proceedings of IIth International Conference on Adaptive Agents and Multi-agent Systems (AAMAS 20I2), Valencia, Spain.

Hennes, D., Claes, D., and Tuyls, K. (2013). Evolutionary advantage of reciprocity in collision avoidance. In Proceedings of the AAMAS 2013 Workshop on Autonomous Robots and Multirobot Systems (ARMS 2013), St. Paul, United States.

Hennes, D., Kaisers, M., and Tuyls, K. (20I0). RESQ-learning in stochastic games. In Proceedings of the AAMAS Workshop on Adaptive and Learning Agents (ALA 20IO) Workshop, Toronto, Canada.

Hennes, D., Tuyls, K., Neerincx, M., and Rauterberg, M. (2009a). Micro-scale social network analysis for ultra-long space flights. In Proceedings of the IJCAI Workshop on Artificial Intelligence in Space (IJCAI 2009), Pasadena, United States.

Hennes, D., Tuyls, K., and Rauterberg, M. (2008). Formalizing multi-state learning dynamics. In IEEE/WIC/ACM International Conference on Web Intelligence and Intelligent Agent Technology (IAT 2008), volume 2, pages 266-272.

Hennes, D., Tuyls, K., and Rauterberg, M. (2009b). State-coupled replicator dynamics. In Proceedings of the 8th International Conference on Autonomous Agents and Multiagent Systems (AAMAS 2009), volume 2, pages 786-796, Budapest, Hungary.

Henrich, J., Boyd, R., Bowles, S., Camerer, C., Fehr, E., and Gintis, H., editors (2004). Foundations of Human Sociality: Economic Experiments and Ethnographic Evidence from Fifteen Small-Scale Societies. Oxford University Press. 
Hoen, P. et al. (2006). An overview of cooperative and competitive multiagent learning. In Proceedings of the Learning and Adaption in Multi-Agent Systems Symposium (LAMAS).

Hofbauer, J. and Sigmund, K. (1998). Evolutionary Games and Population Dynamics. Cambridge University Press.

Hopkins, E. (2002). Two Competing Models of How People Learn in Games. Econometrica, 70(6):2I4I-2I66.

Howard, R. (1960). Dynamic programming and Markov processes. Technology Press of Massachusetts Institute of Technology.

Howell, M. N. and Gordon, T. J. (200I). Continuous action reinforcement learning automata and their application to adaptive digital filter design. Engineering Applications of Artificial Intelligence, I4(5):549-56r.

Hu, J. and Wellman, M. P. (1998). Multiagent reinforcement learning: Theoretical framework and an algorithm. In Proceedings of the Fifteenth International Conference on Machine Learning, pages 242-250. Morgan Kaufmann.

$\mathrm{Hu}$, J. and Wellman, M. P. (2003). Nash Q-learning for general-sum stochastic games. Journal of Machine Learning Research, 4:I039-1069.

Huber, J. (2007). 'J'-shaped returns to timing advantage in access to information - Experimental evidence and a tentative explanation. Journal of Economic Dynamics and Control, 3I(8):2536 - 2572.

Huber, J., Kirchler, M., and Sutter, M. (2008). Is more information always better? Experimental financial markets with cumulative information. Journal of Economic Behavior and Organization, 65(I):86 - I04.

Hughes, T. (2000). The finite element method: linear static and dynamic finite element analysis. Dover Civil and Mechanical Engineering Series. Dover Publications.

Isaacs, R. (1999). Differential Games: A Mathematical Theory With Applications to Warfare and Pursuit, Control and Optimization. Dover Books on Mathematics Series. Dover Publ.

Kaelbling, L., Littman, M., and Moore, A. (1996). Reinforcement learning: A survey. Journal of Artificial Intelligence Research, 4:237-285.

Kaelbling, L. P., Littman, M. L., and Cassandra, A. R. (1998). Planning and acting in partially observable stochastic domains. Artificial Intelligence, IOI(I-2):99-I34. 
Kahneman, D., Slovic, P., and Tversky, A. (I982). Judgment under Uncertainty: Heuristics and Biases. Cambridge University Press.

Kaisers, M. and Tuyls, K. (2010). Frequency adjusted multi-agent Q-learning. In Proceedings of the Ninth International Joint Conferenced on Autonomous Agents and Multiagent Systems, pages 310-316.

Kaisers, M., Tuyls, K., Thuijsman, F., and Parsons, S. (2009). Auction analysis by normal form game approximation. In Web Intelligence and Intelligent Agent Technology, 2008. WI-IAT'O8. IEEE/WIC/ACM International Conference on, volume 2, pages 447-450. IEEE.

Kamar, E., Gal, Y., and Grosz, B. (2009). Modeling user perception of interaction opportunities for effective teamwork. In Symposium on Social Intelligence and Networking, IEEE Conference on Social Computing.

Karandikar, R., Mookherjee, D., Ray, D., and Vega-Redondo, F. (I998). Evolving aspirations and cooperation. Journal of Economic Theory, 80(2):292-33I.

Khan, S. G., Herrmann, G., Lewis, F. L., Pipe, T., and Melhuish, C. (2012). Reinforcement learning and optimal adaptive control: An overview and implementation examples. Annual Reviews in Control, 36(I):42-59.

Kiekintveld, C., Wellman, M. P., and Singh, S. (2006). Empirical game-theoretic analysis of chaturanga. In Proceedings of the AAMAS-o6 Workshop on Game-Theoretic and Decision-Theoretic Agents.

Kirchler, M. (20I0). Partial knowledge is a dangerous thing - On the value of asymmetric fundamental information in asset markets. Journal of Economic Psychology, 2r:643-658.

Klos, T., Van Ahee, G. J., and Tuyls, K. (2010). Evolutionary dynamics of regret minimization. In Proceedings of the 20Io European conference on Machine learning and knowledge discovery in databases: Part II, ECML PKDD 20I0, pages 82-96, Berlin, Heidelberg. Springer-Verlag.

Kolobov, A. (2012). Planning with Markov Decision Processes: An AI Perspective. Morgan \& Claypool.

Könönen, V. (2003). Asymmetric multiagent reinforcement learning. In Proceedings of the IEEE/WIC International Conference on Intelligent Agent Technology, pages 336-. IEEE Computer Society. 
Koutsoupias, E. and Papadimitriou, C. (1999). Worst-case equilibria. In In Proceedings of the I6th annual Symposium on Theoretical Aspects of Computer Science, pages 404$4 \mathrm{I} 3$.

Lachner, R., Breitner, M., and Pesch, H. (2000). Real-time collision avoidance: Differential game, numerical solution, and synthesis of strategies. In Filar, J., Gaitsgory, V., and Mizukami, K., editors, Advances in Dynamic Games and Applications, volume 5 of Annals of the International Society of Dynamic Games, pages II5-I35. Birkhäuser Boston.

Littman, M. L. (1994). Markov games as a framework for multi-agent reinforcement learning. In Proceedings of the Eleventh International Conference on Machine Learning, pages I57-I63. Morgan Kaufmann.

Littman, M. L. (200I). Friend-or-Foe Q-learning in general-sum games. In Proceedings of the Eighteenth International Conference on Machine Learning, pages 322-328. Morgan Kaufmann.

Maes, F., Ernst, D., and Wehenkel, L. (2012). Meta-learning of exploration/exploitation strategies: The multi-armed bandit case. In Proceedings of the 4 th International Conference on Agents and Artificial Intelligence.

Malkiel, B. (2003). The efficient market hypothesis and its critics. Journal of Economic Perspectives, $\mathrm{I} 7(\mathrm{I}): 59-82$.

Matarić, M. J. (1997). Reinforcement learning in the multi-robot domain. Autonomous Robots, 4(I):73-83.

Maynard Smith, J. (1974). The theory of games and the evolution of animal conflicts. Journal of Theoretical Biology, 47(I):209-22I.

Maynard Smith, J. (1982). Evolution and the Theory of Games. Cambridge University Press, Cambridge, UK.

McMillan, J. (1994). Selling spectrum rights. The Journal of Economic Perspectives, 8(3):I45-162.

Messick, D. and Brewer, M. (1983). Solving social dilemmas: A review. Review of personality and social psychology, 4:II-44.

Mitchell, T. (1997). Machine Learning. McGraw-Hill series in Computer Science. McGraw-Hill.

Moody, J., Saffell, M., and Youn, K. (2004). Stochastic direct reinforcement. In AAAI Spring Symposium on Artificial Multiagent Learning, Washington, DC. 
Najim, K. and Poznyak, A. S. (1994). Learning Automata: Theory and Applications. Pergamon, New York.

Narendra, K. and Thathachar, M. (I974). Learning automata: A survey. IEEE Transactions on Systems, Man, and Cybernetics, SMC-I4:323-334.

Narendra, K. and Thathachar, M. (1989). Learning Automata An Introduction. PrenticeHall, Inc., New Jersey.

Nash, J. F. (I950). Equilibrium points in n-person games. Proceedings of the National Academy of Sciences of the United States of America, 36(I):48-49.

Nash, J. F. (I95I). Non-cooperative games. The Annals of Mathematics, 54(2):286-295.

Neyman, A. (2003). From markov chains to stochastic games. In Neyman, A. and Sorin, S., editors, Stochastic Games and Applications, volume 570 of Nato Science Series C: Mathematical and Physical Sciences. Springer.

Nisan, N. and Ronen, A. (1999). Algorithmic mechanism design. In Games and Economic Behavior, pages I29-I40.

Nisan, N., Roughgarden, T., Tardos, E., and Vazirani, V. (2007). Algorithmic Game Theory. Cambridge University Press.

Niu, J., Cai, K., Parsons, S., Fasli, M., and Yao, X. (2012). A grey-box approach to automated mechanism design. Electronic Commerce Research and Applications.

Nowé, A., Verbeeck, K., and Peeters, M. (2006). Learning automata as a basis for multi agent reinforcement learning. In Proceedings of Learning and Adaption in Multi-Agent Systems (LAMAS).

Oliehoek, F. A. (2010). Value-Based Planning for Teams of Agents in Stochastic Partially Observable Environments. PhD thesis, Informatics Institute, University of Amsterdam.

Oosterbeek, H., Sloof, R., and van de Kuilen, G. (2004). Cultural differences in ultimatum game experiments: Evidence from a meta-analysis. Experimental Economics, 7:I7I-I88.

Osborne, M. and Rubinstein, A. (1994). A Course in Game Theory. MIT Press.

Panait, L. and Luke, S. (2005). Cooperative multi-agent learning: The state of the art. Autonomous Agents and Multi-Agent Systems, II(3):387-434. 
Panait, L. and Luke, S. (2006). Selecting informative actions improves cooperative multiagent learning. In Proceedings of the Fifth International Joint Conference on Autonomous Agents and Multiagent Systems. ACM.

Panait, L. and Tuyls, K. (2007). Theoretical advantages of lenient Q-learners: An evolutionary game theoretic perspective. In Proceedings of the International Conference on Autonomous Agents and Multiagent Systems.

Panait, L., Tuyls, K., and Luke, S. (2008). Theoretical advantages of lenient learners: An evolutionary game theoretic perspective. Journal of Machine Learning Research, 9:423-457.

Peeters, M. (2003). A study of reinforcement learning techniques for cooperative multiagent systems. Master's thesis, Vrije Universiteit Brussel.

Phelps, S., Cai, K., McBurney, P., Niu, J., Parsons, S., and Sklar, E. (2007). Auctions, evolution, and multi-agent learning. In Proceedings of the Symposium on Adaptive Learning Agents and Multi-Agent Systems.

Phelps, S., Marcinkiewicz, M., and Parsons, S. (2006). A novel method for automatic strategy acquisition in n-player non-zero-sum games. In Proceedings of the Fifth International Joint Conference on Autonomous Agents and Multiagent Systems, pages 705712. Springer.

Pollatschek, M. and Avi-Itzhak, B. (1969). Algorithms for stochastic games with geometrical interpretation. Management Science, I5(7):399-415.

Ponsen, M., Ramon, J., Croonenborghs, T., Driessens, K., and Tuyls, K. (2008). Bayesrelational learning of opponent models from incomplete information in no-limit poker. In Twenty-third conference of the Association for the Advancement of Artificial Intelligence (AAAI-08).

Ponsen, M., Tuyls, K., Kaisers, M., and Ramon, J. (2009). An evolutionary gametheoretic analysis of poker strategies. Entertainment Computing, I:39-45.

Puterman, M. and Shin, M. (1978). Modified policy iteration algorithms for discounted markov decision problems. Management Science, 24(II):II27-II37.

Puterman, M. L. (2005). Markov decision processes: discrete stochastic dynamic programming. Wiley Series in Probability and Statistics. Wiley-Interscience.

Qiao, H., Rozenblit, J. W., Szidarovszky, F., and Yang, L. (2006). Multi-agent learning model with bargaining. In Proceedings of the Winter Simulation Conference, pages 934-940. 
Rapoport, A. and Chammah, A. M. (I966). The Game of Chicken. American Behavioral Scientist, IO(3):I0-28.

Rebollo, J., Maza, I., and Ollero, A. (2007). Collision avoidance among multiple aerial robots and other non-cooperative aircrafts based on velocity planning. In Proceedings of the 7 th Conference On Mobile Robots And Competitions, Paderne, Portugal.

Roughgarden, T. (2005). Selfish Routing and the Price of Anarchy. MIT Press.

Russell, S. and Norvig, P. (20I0). Artificial Intelligence: A Modern Approach. Prentice Hall Series in Artificial Intelligence. Prentice Hall.

Sandholm, W. (20II). Population Games and Evolutionary Dynamics. Economic Learning and Social Evolution. MIT Press.

Santharam, G., Sastry, P., and Thathachar, M. (1994). Continuous action set learning automata for stochastic optimization. Journal of the Franklin Institute, 33I(5):607 628.

Sen, S. and Weiss, G. (I999). Multiagent learning, chapter 6, pages 259-298. MIT Press, Cambridge, MA, USA.

Shapley, L. S. (1953). Stochastic games. Proceedings of the National Academy of Sciences, 39:1095-IIO0.

Shi, C., Zhang, M., and Peng, J. (2007). Vessel collision avoidance in close-quarter situation using differential games. In Proceedings of the International Conference on Transportation Engineering.

Shoham, Y. (2008). Computer science and game theory. Communications of the Association for Computing Machinery, 5I(8):74-79.

Shoham, Y., Powers, R., and Grenager, T. (2007). If multi-agent learning is the answer, what is the question? Journal of Artificial Intelligence, I7I(7):365-377.

Singh, S., Kearns, M., and Mansour, Y. (2000). Nash convergence of gradient dynamics in general-sum games. In Proceedings of the Sixteenth Conference on Uncertainty in Artificial Intelligence, pages 54I-548. Morgan Kaufmann Publishers Inc.

Skyrms, B. (2003). The Stag Hunt and the Evolution of Social Structure. Cambridge University Press.

Snape, J., van den Berg, J. P., Guy, S. J., and Manocha, D. (20II). The hybrid reciprocal velocity obstacle. IEEE Transactions on Robotics. 
Stimpson, J. L. and Goodrich, M. A. (2003). Learning to cooperate in a social dilemma: A satisficing approach to bargaining. In Proceedings of the Twentieth International Conference on Machine Learning, pages 728-735.

Stone, P. (2007). Multiagent learning is not the answer. It is the question. Artificial Intelligence, I7I:402-05.

Sutton, R. and Barto, A. (1998). Reinforcement Learning: An Introduction. MIT Press, Cambridge, MA.

Sutton, R. S. (I988). Learning to predict by the methods of temporal differences. $M a$ chine Learning, 3(I):9-44.

Sutton, R. S., Barto, A. G., and Williams, R. J. (I99I). Reinforcement learning is direct adaptive optimal control. In Proceedings of the American Control Conference, pages 2143-2146.

Szepesvári, C. (2010). Algorithms for Reinforcement Learning. Synthesis Lectures on Artificial Intelligence and Machine Learning. Morgan \& Claypool.

Tambe, M. (20II). Security and Game Theory: Algorithms, Deployed Systems, Lessons Learned. Cambridge University Press.

Taylor, M. P. and Allen, H. (I992). The use of technical analysis in the foreign exchange market. Journal of International Money and Finance, II(3):304 - 3I4.

Taylor, P. D. and Jonker, L. (1978). Evolutionary stable strategies and game dynamics. Mathematical Biosiences, 40:145-I56.

Tesauro, G. (2003). Extending q-learning to general adaptive multi-agent systems. In Advances in Neural Information Processing Systems I6.

Thathachar, M. and Phansalkar, V. (I995). Learning the global maximum with parameterized learning automata. IEEE Transactions on Neural Networks, 6(2):398-406.

Thathachar, M. and Sastry, P. (2002). Varieties of learning automata: An overview. IEEE Transactions on Systems, Man, and Cybernetics-Part B: Cybernetics, 32(6):7II-722.

Thrun, S. and Littman, M. L. (2000). A review of reinforcement learnaing. Al Magazine, 2I(I):I04-IO5.

Thuijsman, F. (I992). Optimality and equilibria in stochastic games. Centrum voor Wiskunde en Informatica. 
Tóth, B. and Scalas, E. (2007). The value of information in financial markets: An agentbased simulation. In Huber, J. and Hanke, M., editors, Information, Interaction, and (In)Efficiency in Financial Markets. Linde Verlag.

Tóth, B., Scalas, E., Huber, J., and Kirchler, M. (2006). Agent-based simulation of a double-auction market with heterogeneously informed agents. In Potentials of Complexity Science for Business, Governments, and the Media 2006.

Tóth, B., Scalas, E., Huber, J., and Kirchler, M. (2007). The value of information in a multi-agent market model. Eur. Phys. J. B, 55:115-I20.

Tsetlin, M. (I962). On the behavior of finite automata in random media. Autom. Remote Control, 22:1210-I2I9.

Tsitsiklis, J. N. and Roy, B. V. (2002). On average versus discounted reward temporaldifference learning. Machine Learning, 49:I79-I9I.

Tumer, K. and Agogino, A. (2007). Distributed agent-based air traffic flow management. In Proceedings of the 6th International Joint Conference on Autonomous Agents and Multiagent Systems, pages 255:I-255:8, New York, NY, USA. ACM.

Tuyls, K. (2004). Learning in Multi-Agent Systems - An Evolutionary Game Theoretic Approach. PhD thesis, Vrije Universiteit Brussel, Belgium.

Tuyls, K., Heytens, D., Nowe, A., and Manderick, B. (2003a). Extended replicator dynamics as a key to reinforcement learning in multi-agent systems. In Proceedings of the I4th European Conference on Machine Learning (ECML 2003), LNAI Volume: 2837 , Cavtat-Dubrovnik, Croatia.

Tuyls, K. and Parsons, S. (2007). What evolutionary game theory tells us about multiagent learning. Artificial Intelligence, I7I(7):II5-I53.

Tuyls, K., 't Hoen, P., and Vanschoenwinkel, B. (2005). An evolutionary dynamical analysis of multi-agent learning in iterated games. Autonomous Agents and MultiAgent Systems, I2:II5-I53.

Tuyls, K., Verbeeck, K., and Lenaerts, T. (2003b). A selection-mutation model for Qlearning in multi-agent systems. In Proceedings of the International Conference of Autonomous Agents and Multiagent Systems (AAMAS).

Tuyls, K. and Weiss, G. (20I2). Multiagent learning: Basics, challenges, prospects. AI Magazine. 
Tuyls, K. and Westra, R. (2009). Replicator dynamics in discrete and continuous strategy spaces. In Uhrmacher, A., Uhrmacher, A., and Weyns, D., editors, Multi-Agent Systems: Simulation and Applications, Computational Analysis, Synthesis, and Design of Dynamic Systems Series. CRC PressiNC.

Vamvoudakis, K. G. and Lewis, F. L. (20II). Multi-player non-zero-sum games: Online adaptive learning solution of coupled Hamilton-Jacobi equations. Automatica, $47(8): 1556-1569$.

van den Berg, J., Lin, M., and Manocha, D. (2008). Reciprocal velocity obstacles for real-time multi-agent navigation. In ICRA 2008, pages 1928 -I935.

Vohra, R. V. and Wellman, M. P. (2007). Special issue on the foundations of multiagent learning. Artificial Intelligence, $\mathrm{I} 7 \mathrm{I}(\mathrm{I})$.

von Neumann, J. (1928). Zur Theorie der Gesellschaftsspiele. Mathematische Annalen, I00:295-320.

von Neumann, J. and Morgenstern, O. (1944). Theory of games and economic behavior. Princeton University Press, Princeton, NJ.

Vrancx, P., Tuyls, K., Westra, R., and Nowé, A. (2008). Switching dynamics of multiagent learning. In Proceedings of the International Conference on Autonomous Agents and Multiagent Systems (AAMAS).

Vrieze, O. J. (1987). Stochastic games with finite state and action spaces. CWI tract - 33 . Centrum voor Wiskunde en Informatica.

Walsh, W., Parkes, D., and Das, R. (2004). Choosing samples to compute heuristicstrategy Nash equilibrium. Agent-Mediated Electronic Commerce, V:Io9-I23.

Walsh, W. E., Das, R., Tesauro, G., and Kephart, J. O. (2002). Analyzing complex strategic interactions in multi-agent systems. In Proceedings of the Workshop on Game Theoretic and Decision Theoretic Agents, pages I09-II8.

Wang, X. and Sandholm, T. (2002). Reinforcement learning to play an optimal Nash equilibrium in team markov games. In Advances in Neural Information Processing Systems, pages 157I-I578. MIT Press.

Watkins, C. (1989). Learning with Delayed Rewards. PhD thesis, Cambridge University.

Watkins, C. and Dayan, P. (1992). Technical Note: Q-Learning. Machine Learning, 8(3):279-292.

Weibull, J. W. (1995). Evolutionary Game Theory. MIT Press. 
Weibull, J. W. (1997). What have we learned from evolutionary game theory so far? Research Institute of Industrial Economics Working Papers 487, Research Institute of Industrial Economics (IFN).

Weiss, G. (1999). Mutiagent Systems: A Modern Approach to Distributed Artificial Intelligence. Intelligent Robotics and Autonomous Agents Series. Mit Press.

Wheeler, R. and Narendra, K. (1986). Decentralized learning in finite markov chains. IEEE Transactions on Automatic Control, 31:519-526.

White, D. J. (I993). A survey of applications of markov decision processes. The Journal of the Operational Research Society, 44(II):I073-1096.

Xiaohui, J., Xuejun, Z., and Xiangmin, G. (20I2). A collision avoidance method based on satisfying game theory. In Intelligent Human-Machine Systems and Cybernetics (IHMSC), 2012 4th International Conference on, volume 2, pages 96 -99.

Yang, E. and Gu, D. (2005). A survey on multiagent reinforcement learning towards multi-robot systems. In Proceedings of the 2005 IEEE Symposium on Computational Intelligence and Games (ClGo5).

Zinkevich, M. (2003). Online convex programming and generalized infinitesimal gradient ascent. In Proceedings of the Twentieth International Conference on Machine Learning, pages 928-936. AAAI Press. 


\section{Publications}

van den Herik, J. H., Hennes, D., Kaisers, M., Tuyls, K., and Verbeeck, K. (2007). Multiagent learning dynamics: A survey. In Cooperative Information Agents XI (CIA 2007), pages 36-56. Springer.

Hennes, D., Tumer, K., and Tuyls, K. (2008a). A multiagent approach to hyperredundant manipulators. In Proceedings of the AAMAS Workshop on Adaptive Learning Agents and Multi-Agent Systems (ALAMAS+ALAg 2008), Estoril, Portugal.

Hennes, D., Tuyls, K., and Rauterberg, M. (2008b). Formalizing multi-state learning dynamics. In IEEE/WIC/ACM International Conference on Web Intelligence and Intelligent Agent Technology (IAT 2008), volume 2, pages 266-272.

Hennes, D., Tuyls, K., Neerincx, M., and Rauterberg, M. (2009a). Micro-scale social network analysis for ultra-long space flights. In Proceedings of the IJCAI Workshop on Artificial Intelligence in Space (IJCAI 2009), Pasadena, United States.

Hennes, D., Tuyls, K., and Rauterberg, M. (2009b). State-coupled replicator dynamics. In Proceedings of the 8th International Conference on Autonomous Agents and Multiagent Systems (AAMAS 2009), volume 2, pages 786-796, Budapest, Hungary.

Hennes, D., Kaisers, M., and Tuyls, K. (2010). RESQ-learning in stochastic games. In Proceedings of the AAMAS Workshop on Adaptive and Learning Agents (ALA 20IO) Workshop, Toronto, Canada.

Alers, S., Bloembergen, D., Hennes, D., Bügler, M., and Tuyls, K. (20IIa). MITRO: an augmented mobile telepresence robot with assisted control (Demostration). In Proceedings of the gth European Workshop on Multi-agent Systems (EUMAS 20II), Maastricht, Netherlands.

Alers, S., Bloembergen, D., Hennes, D., de Jong, S., Kaisers, M., Lemmens, N., Tuyls, K., and Weiss, G. (20IIb). Bee-inspired foraging in an embodied swarm (Demonstration). In Proceedings of the Ioth International Conference on Autonomous Agents and Multi-Agent Systems (AAMAS 20II), pages I3II-I3I2, Taipei, Taiwan.

Alers, S., Bloembergen, D., Hennes, D., and Tuyls, K. (20IIc). Augmented mobile telepresence with assisted control (Demonstration). In Proceedings of 23rd Benelux Conference on Artificial Intelligence (BNAIC 2OII), pages 45I-452, Gent, Belgium. 
Claes, D., Hennes, D., and Tuyls, K. (20Ira). Real-world multi-robot collision avoidance with localization uncertainty. In Proceedings of gth European Workshop on Multiagent Systems (EUMAS 20II), Maastricht, Netherlands.

Claes, D., Hennes, D., and Tuyls, K. (20Irb). Real-world multi-robot collision avoidance with localization uncertainty (Demonstration). In Proceedings of 9 th European Workshop on Multi-agent Systems (EUMAS 2OII), Maastricht, Netherlands.

de Jong, S., Hennes, D., Tuyls, K., and Gal, Y. (20Ira). Meta-strategies in the colored trails game. In Proceedings of the Ioth International Conference on Autonomous Agents and Multi-Agent Systems (AAMAS 20II), pages 55I-558, Taipei, Taiwan.

de Jong, S., Hennes, D., Tuyls, K., and Gal, Y. (20IIb). Meta-strategies in the colored trails game. In Proceedings of 23 rd Benelux Conference on Artificial Intelligence (BNAIC 20II), pages 383-384, Gent, Belgium.

Wurm, K. M., Hennes, D., Holz, D., Rusu, R. B., Stachniss, C., Konolige, K., and Burgard, W. (2OII). Hierarchies of octrees for efficient 3d mapping. In Proceedings of IEEE/RSJ International Conference on Intelligent Robots and Systems (IROS 2OII), pages 42494255, San Francisco, United States.

Alers, S., Bloembergen, D., Bügler, M., Hennes, D., and Tuyls, K. (20I2). MITRO: an augmented mobile telepresence robot with assisted control (Demostration). In Proceedings of IIth International Conference on Adaptive Agents and Multi-agent Systems (AAMAS 20I2), Valencia, Spain.

Claes, D., Hennes, D., Meeussen, W., and Tuyls, K. (2012a). CALU: Multi-robot collision avoidance with localization uncertainty (Demonstration). In Proceedings of IIth International Conference on Adaptive Agents and Multi-agent Systems (AAMAS 20I2), Valencia, Spain.

Claes, D., Hennes, D., and Tuyls, K. (20I2b). COCALU: Convex outline collision avoidance under localization uncertainty (Demonstration). In Proceedings of the 24th Benelux Conference on Artificial Intelligence (BNAIC 20I2), Maastricht, Netherlands.

Claes, D., Hennes, D., Tuyls, K., and Meeussen, W. (20I2c). Collision avoidance under bounded localization uncertainty. In Proceedings of IEEE/RSJ International Conference on Intelligent Robots and Systems (IROS 20I2), Vilamoura, Portugal.

Hennes, D., Bloembergen, D., Kaisers, M., Tuyls, K., and Parsons, S. (2012a). Evolutionary advantage of foresight in markets. In Proceedings of the Genetic and Evolutionary Computation Conference (GECCO 20I2), pages 943-949, Philadelphia, United States. 
Hennes, D., Claes, D., Meeussen, W., and Tuyls, K. (20I2b). Multi-robot collision avoidance with localization uncertainty. In Proceedings of IIth International Conference on Adaptive Agents and Multi-agent Systems (AAMAS 20I2), Valencia, Spain.

Alers, S., Bloembergen, D., Claes, D., Fossel, J., Hennes, D., and Tuyls, K. (2013). Telepresence robots as a research platform for AI. In Proceedings of the AAAI Spring Symposium on Designing Intelligent Robots: Reintegrating AI II.

Fossel, J., Hennes, D., Alers, S., Claes, D., and Tuyls, K. (2013a). OctoSLAM: A 3D mapping approach to situational awareness of unmanned aerial vehicles. In Proceedings of the 2013 International Conference on Unmanned Aircraft Systems (ICUAS 20I3), Atlanta, United States.

Fossel, J., Hennes, D., Alers, S., Claes, D., and Tuyls, K. (2013b). OctoSLAM: A 3D mapping approach to situational awareness of unmanned aerial vehicles (Demonstration). In Proceedings of I2th International Conference on Adaptive Agents and Multiagent Systems (AAMAS 2013), St. Paul, United States.

Hennes, D., Claes, D., and Tuyls, K. (2013). Evolutionary advantage of reciprocity in collision avoidance. In Proceedings of the AAMAS 2013 Workshop on Autonomous Robots and Multirobot Systems (ARMS 20I3), St. Paul, United States. 



\section{List of Figures}

3.I Symmetric replicator dynamics of the prisoners' dilemma $\diamond 42$

3.2 Symmetric replicator dynamics in the game of rock-paper-scissors $\diamond 43$

3.3 Asymmetric replicator dynamics $\diamond 44$

3.4 Asymmetric replicator dynamics of the Bach or Stravinsky game $\diamond 50$

3.5 Dynamics in the single state prisoners' dilemma $\diamond 5 \mathrm{I}$

3.6 Dynamics in the Bach or Stravinsky game $\diamond 52$

3.7 Dynamics in the matching pennies game $\diamond 52$

4.I Trajectory plot for two-state prisoners' dilemma (I) $\diamond 66$

4.2 Trajectory plot for two-state prisoners' dilemma $(2) \diamond 67$

4.3 Cell partitioning for vector field sensitivity analysis $\diamond 68$

4.4 Vector field sensitivity analysis $\diamond 69$

4.5 LA, PWRD, and SCRD in the two-state prisoners' dilemma (I) $\diamond 72$

4.6 LA, PWRD, and SCRD in the two-state prisoners' dilemma (2) $\diamond 73$

4.7 LA, PWRD, and SCRD in the common interest game $\diamond 74$

4.8 Symmetric replicator dynamics in the two-state prisoners' dilemma $\diamond 75$

4.9 RESQ-learning dynamics in the two-state prisoners' dilemma $\diamond 79$

4.Io RESQ-learning in the two-state matching pennies game $\diamond 79$

4.II Comparison between SC-RD, RESQ dynamics and RESQ-learning $\diamond 80$

5.I Example Colored Trails game instances and gain graphs $\diamond 88$

5.2 Extensive-form representation of the three-player negotiation variant $\diamond 9 \mathrm{I}$

5.3 Pure or mixed strategies on the convex hull of the gain graph $\diamond 93$

5.4 Effect of inequity aversion on the utility of the responder $\diamond 95$

5.5 Single population dynamics in the three-strategy game $\diamond$ IO2

5.6 Single population dynamics with inequity aversion $\diamond$ IO3

6.I A Brownian motion dividend stream $\diamond$ Io8

6.2 Relative market return over information level $\diamond$ III

6.3 Relative market return for various information distributions $\diamond \quad$ II2

6.4 Relative market return over information levels 0 , 3, and $9 \diamond \mathrm{II} 3$

6.5 Evolutionary market dynamics $\diamond$ II5

6.6 Evolutionary market dynamics using a fixed and quadratic cost $\diamond$ II6 
7.I Symmetric replicator dynamics of the game of chicken $\diamond$ I2I

7.2 Velocity obstacles $\diamond \mathrm{I} 22$

7.3 Truncation of a velocity obstacles $\diamond \quad$ I24

7.4 ClearPath algorithm $\diamond$ I25

7.5 Trajectories of 6 RVO agents $\diamond$ I26

7.6 Evolutionary dynamics of collision avoidance $\diamond \quad$ I26

7.7 Evolutionary dynamics of collision avoidance with truncation $\diamond \quad$ I27 


\section{List of Tables}

I.I Payoff table of the prisoners' dilemma $\diamond 3$

I.2 Payoff table of the matching pennies game $\diamond 4$

3.I Payoff table of the Bach or Stravinsky game $\diamond 50$

5.I Induced two-proposer game with two metastrategies $\diamond 92$

5.2 Induced two-proposer CT game with three metastrategies $\diamond 94$

5.3 Payoff matrix for CT game with one proposer and one responder $\diamond \quad 97$

5.4 Payoff matrix for CT game with one proposer and $m$ responders $\diamond \quad 97$

5.5 Empirical payoff matrix for the CT game with two metastrategies $\diamond$ Ioo

5.6 Empirical payoff matrix for the CT game with three metastrategies $\diamond$ Ioo

5.7 Empirical payoff matrix with inequity aversion (unaware proposers) $\diamond$ IOI

5.8 Empirical payoff matrix with inequity aversion (aware proposers) $\diamond$ IOI

7.I Payoff table of the game of chicken $\diamond$ I2O 



\section{Summary}

More and more contemporary technological challenges require decentralized solutions. Examples include the coordination of multiple autonomous vehicles and automated control systems for air traffic. The demand for multiagent systems is widespread and originates from a collection of significant advantages. Multiagent systems have built-in redundancies that allow for robustness, i.e., if one or a few components fail the system is still able to operate properly. In addition, different tasks can be assigned to independent agents pursuing these goals in parallel. Multiagent systems are modular per definition and components can be easily added or removed. While a multiagent approach offers an elegant solution paradigm to issues like fault tolerance and load balancing, it also introduces new challenges. The presence of multiple agents results in a highly dynamic and nondeterministic environment. Interactions between agents and with the environment inevitably cause very complex systems, which are not trivial to control. In order to facilitate adaptation to changes in the environment and other agents, learning is crucial.

This dissertation investigates the dynamics of learning in multiagent systems and is organized in two parts. The first part investigates evolutionary game theory as a formal framework to model the dynamics of multiagent learning. The second part focuses on the application of these models to real world problems. Part I commences with the formal concepts and key results of multiagent learning. We define the fundamental models of single- and multiagent learning and give an introduction to two well-known reinforcement learning algorithms, $Q$-learning and learning automata. Subsequently, we survey the most important multiagent learning algorithms. We continue by explaining the relation between evolutionary game theory and multiagent reinforcement learning. In particular, we show the formal relation between games of learning automata and the asymmetric continuous time replicator dynamics, as well as the link between $Q$-learning and the selection-mutation dynamics. This dissertation makes two important contributions to the methodology of modeling multiagent reinforcement learning by evolutionary processes. First, we prove that a large collective of learning agents can be approximated by the symmetric continuous time replicator dynamics. Second, we extend the link between multiagent reinforcement learning and evolutionary game theory to multi-state games.

Part II of this dissertation focuses on the dynamics of multiagent learning in real world domains. In particular, we investigate three domains: complex negotiation scenarios, markets, and collision avoidance. Complex negotiations involve strategic interactions in settings that include multiple agents and multiple actions as well as interrelated goals, tasks and resources. As the complexity of these large-scale strategic inter- 
actions grows exponentially with the number of agents, we introduce metastrategies as a method to reduce the strategy space significantly. We show that we can preserve many of the strategic characteristics in the reduced setting, which makes it possible to use population games to model the dynamics of such interactions. The competitive advantage of price signal information for traders in auctions is the subject of the second domain study. More information does not guarantee higher performance, in particular, traders with limited information perform below market average and are outperformed by random traders; only insiders beat the market. We analyze the market dynamics with an evolutionary model of agents with competing information levels. Results show that the highest information level will dominate if information comes for free. If information is costly, less-informed traders may prevail reflecting a more realistic distribution over information levels. In the third domain, we perform an evolutionary analysis of collision avoidance in multiagent systems based on the velocity obstacle paradigm. The velocity obstacle is a geometric representation of all velocities that will eventually result in a collision given that all dynamic obstacles maintain their observed velocities. Three variants of the velocity obstacle description are compared and the most robust technique under evolutionary pressure is identified.

To conclude, this dissertation contributes to the understanding of multiagent learning dynamics in several ways. First, we provide an alternative interpretation of the single population replicator dynamics. In particular, we derive that if fitness proportionate selection is used, the overall population average of a population of learners moves independently of the adaptation process of individuals; it follows the symmetric continuous time replicator dynamics. Second, we derive a dynamical model of multi-state multiagent learning. The state-coupled replicator dynamics extend the existing evolutionary framework of multiagent learning and allow to predict learning dynamics in stochastic games. Finally, we show how evolutionary models of learning can be used to analyze complex scenarios and predict behavior, thereby linking the theoretical concepts to everyday problems.

About the author: Daniel Hennes was born on July 4th, I984 in Duisburg, Germany. He graduated summa cum laude from Maastricht University with a B.Sc. in Knowledge Engineering and Computer Science (August 2007) and a M.Sc. in Artificial Intelligence (June 2008). In July 2008, he started his Ph.D. research at Eindhoven University of Technology. From September 2009 onwards, he continued his research at the Department of Knowledge Engineering at Maastricht University. From September 2007 to January 2008 and February 2010 to June 2010, he worked at Oregon State University as a visiting research scholar in the area of Dynamics and Control. From September 2010 to December 20Io, he was a research intern at Willow Garage, a robotics research lab and technology incubator in Menlo Park, California. 


\section{Samenvatting}

Steeds meer hedendaagse technologische uitdagingen vereisen decentrale oplossingen. Voorbeelden hiervan zijn onder andere de coördinatie van meerdere autonome voertuigen en geautomatiseerde regelsystemen voor het luchtverkeer. De vraag naar multi-agentsystemen is wijdverbreid en komt voort uit enkele belangrijke voordelen. Multi-agentsystemen hebben ingebouwde redundanties die leiden tot robuustheid; als één of enkele onderdelen falen is het systeem nog steeds in staat om goed te functioneren. Daarnaast kunnen verschillende taken worden toegewezen aan individuele agenten die deze doelen parallel nastreven. Multi-agentsystemen zijn per definitie modulair en componenten kunnen eenvoudig worden toegevoegd of verwijderd. Naast het feit dat multi-agentsystemen een elegante oplossingsmethode bieden voor problemen zoals fouttolerantie en load balancing, worden er ook nieuwe uitdagingen geïntroduceerd. De aanwezigheid van meerdere agenten leidt tot een zeer dynamische en complex omgeving. Interacties tussen agenten en met de omgeving leiden onvermijdelijk tot zeer complexe systemen die niet triviaal te controleren zijn. Om aanpassing aan veranderingen in de omgeving en andere agenten te vergemakkelijken, is leren van groot belang.

Dit proefschrift onderzoekt de dynamiek van leergedrag in multi-agentsystemen en is georganiseerd in twee delen. In het eerste deel onderzoeken we evolutionaire speltheorie als een formeel kader om de dynamica van multiagent learning te modelleren. In het tweede deel richten we ons op de toepassing van deze modellen binnen praktische domeinen. Deel I begint met de formele definities en de belangrijkste resultaten met betrekking tot multiagent learning. We introduceren de fundamentele modellen van single- en multiagent learning en geven een inleiding tot twee bekende reinforcement learning algoritmen, Q-learning en learning automata. Hierna geven we een overzicht van de belangrijkste algoritmen voor multiagent learning. Vervolgens zetten we de relatie tussen evolutionaire speltheorie en multiagent reinforcement learning uiteen. In het bijzonder tonen we de formele relatie aan tussen het leergedrag van learning automata en de asymmetric continuous time replicator dynamics, alsmede het verband tussen $Q$-learning en selectie-mutatie dynamica. Dit proefschrift draagt op twee manieren bij aan de methodiek van het modelleren van multiagent reinforcement learning doormiddel van evolutionaire processen. Ten eerste bewijzen we dat een grote populatie van lerende agenten kan worden benaderd door de symmetric continuous time replicator dynamics. Ten tweede breiden we de relatie tussen multiagent reinforcement learning en evolutionaire speltheorie uit naar multi-state games.

Deel Il van dit proefschrift richt zich op de toepassing van deze dynamische modellen binnen praktische domeinen. We onderzoeken drie domeinen: complexe on- 
derhandelingsscenario's, aandelenmarkten, en collision avoidance. Complexe onderhandelingen zijn strategische interacties in scenario's die meerdere agenten, vele mogelijke acties, en verbanden tussen doelen, taken en middelen bevatten. Omdat de complexiteit van deze strategische interacties exponentieel groeit met het aantal agenten, introduceren we meta-strategies als een methode om de complexiteit beduidend in te perken. We laten zien dat veel van de strategische kenmerken van het originele scenario behouden blijven binnen deze gereduceerde representatie, wat ons in staat stelt om de dynamiek van deze interacties te modelleren met behulp van evolutionaire spellen. Het competitief voordeel van prijssignaal-informatie voor handelaren op de aandelenmarkt is het onderwerp van de tweede praktische toepassing. Het hebben van meer informatie is geen garantie voor betere prestaties, zo presteren met name handelaren met beperkte informatie onder het marktgemiddelde en worden zij overtroffen door handelaren die willekeurig opereren; alleen insiders zijn in staat om de markt te verslaan. We analyseren de dynamiek van de aandelenmarkt met een evolutionair model van agenten met verschillende informatieniveaus. Uit de resultaten blijkt dat het hoogste informatieniveau zal domineren zolang informatie vrij beschikbaar is. Als informatie kostbaar is, kunnen ook minder geïnformeerde handelaren voortbestaan, met een meer realistische verdeling over informatieniveaus tot gevolg. Tot slot voeren we een evolutionaire analyse uit van collision avoidance in multi-agentsystemen op basis van de velocity obstacle methode. Het velocity obstacle is een geometrische weergave van alle snelheden die uiteindelijk tot een botsing zullen leiden, aangenomen dat het dynamische obstakel zijn waargenomen snelheid handhaaft. Drie varianten van de velocity obstacle methode worden vergeleken en de meest robuuste aanpak wordt bepaald middels een aantal experimenten.

Concluderend, dit proefschrift draagt op meerdere manieren bij aan een beter begrip van de dynamiek van multiagent learning. Ten eerste bieden we een alternatieve interpretatie van de single population replicator dynamics. In het bijzonder leiden we af dat, wanneer selectie proportioneel aan prestatie plaatsvindt, het gemiddelde gedrag van een populatie lerende agenten niet afhankelijk is van het gedrag van de individuele agenten, maar de single population replicator dynamics volgt. Ten tweede leiden we een dynamisch model af van multi-state multi-agent learning. De state-coupled replicator dynamics verbreden de evolutionaire methodiek van multiagent learning en stellen ons in staat om leerdynamica in stochastische spellen te voorspellen. Tot slot laten we zien hoe evolutionaire modellen van leergedrag kunnen worden gebruikt om complexe systemen te analyseren en gedrag te voorspellen, waarmee we een brug slaan tussen de theoretische concepten en problemen uit het dagelijks leven. 


\section{Acknowledgements}

First and foremost, I would like to thank Karl Tuyls for his advice, guidance and encouragement as well as his continued support and confidence in me. Karl has been a great mentor, very resourceful and he always made an effort to introduce me to members of the scientific community. It is very reassuring to have someone to turn to whenever advice is needed, professional as well as personal. Second, I would like to thank Kagan Tumer. Oregon State University has become my second academic home, with two prolonged research visits in the past years. I am very grateful for this exceedingly instructive and enjoyable experience. I would also like to thank Gerhard Weiss for his advice, kind encouragements and support in the final phase of this research endeavour. Furthermore, I would like to express my gratitude to the assessment committee members Ralf Peeters, Robert Babuska, Kurt Driessens, Jan Scholtes, and Milind Tambe for their efforts and valuable feedback.

Many thanks to my dear friends Michael Kaisers and Daan Bloembergen. I can always rely on their support, collaboration, critical feedback and great company. My great appreciation goes to Michael, Daan and Jörn Martin Horschig for proofreading this manuscript. I would like to thank Matt Knudson and Atil Iscen for the many interesting discussions and for welcoming me so kindly at Oregon State University. Furthermore, Jesse, Cecile, Anna, Miriam, Christophe, and William deserve a big "thank you!" for making my visit to Oregon such a pleasant experience. I had an amazing time as a research intern at Willow Garage in sunny Menlo Park, California. What better place to soak up some of that Silicon Valley entrepreneurial spirit and get in touch with people that have a tireless drive for innovation? Special thanks to Wim Meeussen, Troy Straszheim, and my fellow interns: Christian Connette, Dirk Holz, Matthew Robards, Hauke Strasdat and Kai Wurm. I also like to thank my colleagues, office mates (present and past), and fellow PhD candidates in Maastricht: Sjriek Alers, Hendrik Baier, Haitham Bou Ammar, Daniel Claes, Joscha Fossel, Steven de Jong, Nyree Lemmens, Mark Ponsen, and Philippe Uyttendaele. Thank you for your friendship, fruitful collaborations, interesting discussions and a very pleasant working environment.

Whatever lies ahead - I will always look back fondly on my time in Maastricht. This is the place where I met some truly great people. I especially thank Gesa, Sam, Marc, Max, Cherie, Kathi, Alejandro, Francesca, Fabian, and Tobias, for all the great memories. I am also very grateful for my friendship with Niels, Martin, Matthias, Caroline and Claudia. Although we do not see each other as much as I would like to, when we do they never fail to put a smile on my face. Finally, I would like to thank my family and Pia. They deserve all my gratitude for their support, encouragement, and joyful presence in my life.

Daniel Hennes, April 2013. 



\section{SIKS Dissertation Series}

2009

I Rasa Jurgelenaite (RUN) Symmetric Causal Independence Models

2 Willem Robert van Hage (VU) Evaluating Ontology-Alignment Techniques

3 Hans Stol (UvT) A Framework for Evidence-based Policy Making Using IT

4 Josephine Nabukenya (RUN) Improving the Quality of Organisational Policy Making using Collaboration Engineering

5 Sietse Overbeek (RUN) Bridging Supply and Demand for Knowledge Intensive Tasks - Based on Knowledge, Cognition, and Quality

6 Muhammad Subianto (UU) Understanding Classification

7 Ronald Poppe (UT) Discriminative Vision-Based Recovery and Recognition of Human Motion

8 Volker Nannen (VU) Evolutionary Agent-Based Policy Analysis in Dynamic Environments

9 Benjamin Kanagwa (RUN) Design, Discovery and Construction of Service-oriented Systems

Io Jan Wielemaker (UVA) Logic programming for knowledge-intensive interactive applications

II Alexander Boer (UVA) Legal Theory, Sources of Law \& the Semantic Web

I2 Peter Massuthe (TUE) Humboldt-Universitaet BerlinOperating Guidelines for Services

I3 Steven de Jong (UM) Fairness in Multi-Agent Systems

I4 Maksym Korotkiy (VU) From ontology-enabled services to service-enabled ontologies (making ontologies work in e-science with ONTO-SOA)

15 Rinke Hoekstra (UVA) Ontology Representation Design Patterns and Ontologies that Make Sense
I6 Fritz Reul (UvT) New Architectures in Computer Chess

I7 Laurens van der Maaten (UvT) Feature Extraction from Visual Data

I8 Fabian Groffen (CWI) Armada, An Evolving Database System

I9 Valentin Robu (CWI) Modeling Preferences, Strategic Reasoning and Collaboration in Agent-Mediated Electronic Markets

20 Bob van der Vecht (UU) Adjustable Autonomy: Controling Influences on Decision Making

2I Stijn Vanderlooy (UM) Ranking and Reliable Classification

22 Pavel Serdyukov (UT) Search For Expertise: Going beyond direct evidence

23 Peter Hofgesang (VU) Modelling Web Usage in a Changing Environment

24 Annerieke Heuvelink (VU) Cognitive Models for Training Simulations

25 Alex van Ballegooij (CWI) RAM: Array Database Management through Relational Mapping

26 Fernando Koch (UU) An Agent-Based Model for the Development of Intelligent Mobile Services

27 Christian Glahn (OU) Contextual Support of social Engagement and Reflection on the Web

28 Sander Evers (UT) Sensor Data Management with Probabilistic Models

29 Stanislav Pokraev (UT) Model-Driven Semantic Integration of Service-Oriented Applications

30 Marcin Zukowski (CWI) Balancing vectorized query execution with bandwidth-optimized storage

3I Sofiya Katrenko (UVA) A Closer Look at Learning Relations from Text

\footnotetext{
Abbreviations: SIKS - Dutch Research School for Information and Knowledge Systems; CWI - Centrum voor Wiskunde en Informatica, Amsterdam; EUR - Erasmus Universiteit, Rotterdam; KUB - Katholieke Universiteit Brabant, Tilburg; KUN - Katholieke Universiteit Nijmegen; RUG - Rijksuniversiteit Groningen; RUL - Rijksuniversiteit Leiden; RUN - Radboud Universiteit Nijmegen; TUD - Technische Universiteit Delft; TU/e - Technische Universiteit Eindhoven; UL - Universiteit Leiden; UM - Universiteit Maastricht; UT - Universiteit Twente; UU - Universiteit Utrecht; UvA - Universiteit van Amsterdam; UvT - Universiteit van Tilburg; VU - Vrije Universiteit, Amsterdam.
} 
32 Rik Farenhorst and Remco de Boer (VU) Architectural Knowledge Management: Supporting Architects and Auditors

33 Khiet Truong (UT) How Does Real Affect Affect Affect Recognition In Speech?

34 Inge van de Weerd (UU) Advancing in Software Product Management: An Incremental Method Engineering Approach

35 Wouter Koelewijn (UL) Privacy en Politiegegevens; Over geautomatiseerde normatieve informatieuitwisseling

36 Marco Kalz (OUN) Placement Support for Learners in Learning Networks

37 Hendrik Drachsler (OUN) Navigation Support for Learners in Informal Learning Networks

38 Riina Vuorikari (OU) Tags and self-organisation: a metadata ecology for learning resources in a multilingual context

39 Christian Stahl (TUE, Humboldt-Universitaet zu Berlin) Service Substitution - A Behavioral Approach Based on Petri Nets

40 Stephan Raaijmakers (UvT) Multinomial Language Learning: Investigations into the Geometry of Language

4I Igor Berezhnyy (UvT) Digital Analysis of Paintings

42 Toine Bogers (UvT) Recommender Systems for Social Bookmarking

43 Virginia Nunes Leal Franqueira (UT) Finding Multi-step Attacks in Computer Networks using Heuristic Search and Mobile Ambients

44 Roberto Santana Tapia (UT) Assessing Business-IT Alignment in Networked Organizations

45 Jilles Vreeken (UU) Making Pattern Mining Useful

46 Loredana Afanasiev (UvA) Querying XML: Benchmarks and Recursion

\section{0}

I Matthijs van Leeuwen (UU) Patterns that Matter

2 Ingo Wassink (UT) Work flows in Life Science

3 Joost Geurts (CWI)A Document Engineering Model and Processing Framework for Multimedia documents
4 Olga Kulyk (UT) Do You Know What I Know? Situational Awareness of Co-located Teams in Multidisplay Environments

5 Claudia Hauff (UT) Predicting the Effectiveness of Queries and Retrieval Systems

6 Sander Bakkes (UvT) Rapid Adaptation of Video Game AI

7 Wim Fikkert (UT) Gesture interaction at a Distance

8 Krzysztof Siewicz (UL) Towards an Improved Regulatory Framework of Free Software. Protecting user freedoms in a world of software communities and eGovernments

9 Hugo Kielman (UL) A Politiele gegevensverwerking en Privacy, Naar een effectieve waarborging

Io Rebecca Ong (UL) Mobile Communication and Protection of Children

II Adriaan Ter Mors (TUD) The world according to MARP: Multi-Agent Route Planning

I2 Susan van den Braak (UU) Sensemaking software for crime analysis

I3 Gianluigi Folino (RUN) High Performance Data Mining using Bio-inspired techniques

I4 Sander van Splunter (VU) Automated Web Service Reconfiguration

I5 Lianne Bodenstaff (UT) Managing Dependency Relations in Inter-Organizational Models

I6 Sicco Verwer (TUD) Efficient Identification of Timed Automata, theory and practice

I7 Spyros Kotoulas (VU) Scalable Discovery of Networked Resources: Algorithms, Infrastructure, Applications

I8 Charlotte Gerritsen (VU) Caught in the Act: Investigating Crime by Agent-Based Simulation

I9 Henriette Cramer (UvA) People's Responses to Autonomous and Adaptive Systems

20 Ivo Swartjes (UT) Whose Story Is It Anyway? How Improv Informs Agency and Authorship of Emergent Narrative

2I Harold van Heerde (UT) Privacy-aware data management by means of data degradation

22 Michiel Hildebrand (CWI) End-user Support for Access to Heterogeneous Linked Data 
23 Bas Steunebrink (UU) The Logical Structure of Emotions

24 Dmytro Tykhonov () Designing Generic and Efficient Negotiation Strategies

25 Zulfiqar Ali Memon (VU) Modelling HumanAwareness for Ambient Agents: A Human Mindreading Perspective

26 Ying Zhang (CWI) XRPC: Efficient Distributed Query Processing on Heterogeneous XQuery Engines

27 Marten Voulon (UL) Automatisch contracteren

28 Arne Koopman (UU) Characteristic Relational Patterns

29 Stratos Idreos (CWI) Database Cracking: Towards Auto-tuning Database Kernels

30 Marieke van Erp (UvT) Accessing Natural History - Discoveries in data cleaning, structuring, and retrieval

3I Victor de Boer (UVA) Ontology Enrichment from Heterogeneous Sources on the Web

32 Marcel Hiel (UvT) An Adaptive Service Oriented Architecture: Automatically solving Interoperability Problems

33 Robin Aly (UT) Modeling Representation Uncertainty in Concept-Based Multimedia Retrieval

34 Teduh Dirgahayu (UT) Interaction Design in Service Compositions

35 Dolf Trieschnigg (UT) Proof of Concept: Conceptbased Biomedical Information Retrieval

36 Jose Janssen (OU) Paving the Way for Lifelong Learning; Facilitating competence development through a learning path specification

37 Niels Lohmann (TUE) Correctness of services and their composition

38 Dirk Fahland (TUE) From Scenarios to components

39 Ghazanfar Farooq Siddiqui (VU) Integrative modeling of emotions in virtual agents

40 Mark van Assem (VU) Converting and Integrating Vocabularies for the Semantic Web

4I Guillaume Chaslot (UM) Monte-Carlo Tree Search

42 Sybren de Kinderen (VU) Needs-driven service bundling in a multi-supplier setting - the computational e3-service approach
43 Peter van Kranenburg (UU) A Computational Approach to Content-Based Retrieval of Folk Song Melodies

44 Pieter Bellekens (TUE) An Approach towards Context-sensitive and User-adapted Access to Heterogeneous Data Sources, Illustrated in the Television Domain

45 Vasilios Andrikopoulos (UvT) A theory and model for the evolution of software services

46 Vincent Pijpers (VU) ezalignment: Exploring InterOrganizational Business-ICT Alignment

47 Chen Li (UT) Mining Process Model Variants: Challenges, Techniques, Examples

48 Jahn-Takeshi Saito (UM) Solving difficult game positions

49 Bouke Huurnink (UVA) Search in Audiovisual Broadcast Archives

50 Alia Khairia Amin (CWI) Understanding and supporting information seeking tasks in multiple sources

5I Peter-Paul van Maanen (VU) Adaptive Support for Human-Computer Teams: Exploring the Use of Cognitive Models of Trust and Attention

52 Edgar Meij (UVA) Combining Concepts and Language Models for Information Access

$20 I I$

I Botond Cseke (RUN) Variational Algorithms for Bayesian Inference in Latent Gaussian Models

2 Nick Tinnemeier (UU) Work flows in Life Science

3 Jan Martijn van der Werf(TUE) Compositional Design and Verification of Component-Based Information Systems

4 Hado van Hasselt (UU) Insights in Reinforcement Learning; Formal analysis and empirical evaluation of temporal-difference learning algorithms

5 Base van der Raadt (VU) Enterprise Architecture Coming of Age - Increasing the Performance of an Emerging Discipline.

6 Yiwen Wang (TUE) Semantically-Enhanced Recommendations in Cultural Heritage

7 Yujia Cao (UT) Multimodal Information Presentation for High Load Human Computer Interaction 
8 Nieske Vergunst (UU) BDI-based Generation of Robust Task-Oriented Dialogues

9 Tim de Jong (OU) Contextualised Mobile Media for Learning

Io Bart Bogaert (UvT) Cloud Content Contention

II Dhaval Vyas (UT) Designing for Awareness: An Experience-focused $\mathrm{HCl}$ Perspective

I2 Carmen Bratosin (TUE) Grid Architecture for Distributed Process Mining

I3 Xiaoyu Mao (UvT) Airport under Control. Multiagent Scheduling for Airport Ground Handling

I4 Milan Lovric (EUR) Behavioral Finance and AgentBased Artificial Markets

I5 Marijn Koolen (UvA) The Meaning of Structure: the Value of Link Evidence for Information Retrieval

I6 Maarten Schadd (UM) Selective Search in Games of Different Complexity

I7 Jiyin He (UVA) Exploring Topic Structure: Coherence, Diversity and Relatedness

I8 Mark Ponsen (UM) Strategic Decision-Making in complex games

I9 Ellen Rusman (OU) The Mind's Eye on Personal Profiles

20 Qing $\mathrm{Gu}(\mathrm{VU})$ Guiding service-oriented software engineering - A view-based approach

2I Linda Terlouw (TUD) Modularization and Specifcation of Service-Oriented Systems

22 Junte Zhang (UVA) System Evaluation of Archival Description and Access

23 Wouter Weerkamp (UVA) Finding People and their Utterances in Social Media

24 Herwin van Welbergen (UT) Behavior Generation for Interpersonal Coordination with Virtual Humans On Specifying, Scheduling and Realizing Multimodal Virtual Human Behavior

25 Syed Waqar ul Qounain Jaffry (VU) Analysis and Validation of Models for Trust Dynamics

26 Matthijs Aart Pontier (VU) Virtual Agents for Human Communication - Emotion Regulation and Involvement-Distance Trade-Offs in Embodied Conversational Agents and Robots

27 Aniel Bhulai (VU) Dynamic website optimization through autonomous management of design patterns
28 Rianne Kaptein (UVA) Effective Focused Retrieval by Exploiting Query Context and Document Structure

29 Faisal Kamiran (TUE) Discrimination-aware Classification

30 Egon van den Broek (UT) Affective Signal Processing (ASP): Unraveling the mystery of emotions

3I Ludo Waltman (EUR) Computational and GameTheoretic Approaches for Modeling Bounded Rationality

32 Nees-Jan van Eck (EUR) Methodological Advances in Bibliometric Mapping of Science

33 Tom van der Weide (UU) Arguing to Motivate Decisions

34 Paolo Turrini (UU) Strategic Reasoning in Interdependence: Logical and Game-theoretical Investigations

35 Maaike Harbers (UU) Explaining Agent Behavior in Virtual Training

36 Erik van der Spek (UU) Experiments in serious game design: a cognitive approach

37 Adriana Birlutiu (RUN) Machine Learning for Pairwise Data, Applications for Preference Learning and Supervised Network Inference

38 Nyree Lemmens (UM) Bee-inspired Distributed Optimization

39 Joost Westra (UU) Organizing Adaptation using Agents in Serious Games

40 Viktor Clerc (VU) Architectural Knowledge Management in Global Software Development

4I Luan Ibraimi (UT) Cryptographically Enforced Distributed Data Access Control

42 Michal Sindlar (UU) Explaining Behavior through Mental State Attribution

43 Henk van der Schuur (UU) Process Improvement through Software Operation Knowledge

44 Boris Reuderink (UT) Robust Brain-Computer Interfaces

45 Herman Stehouwer (UvT) Statistical Language Models for Alternative Sequence Selection

46 Beibei Hu (TUD) Towards Contextualized Information Delivery: A Rule-based Architecture for the Domain of Mobile Police Work 
47 Azizi Bin Ab Aziz (VU) Exploring Computational Models for Intelligent Support of Persons with Depression

48 Mark Ter Maat (UT) Response Selection and Turntaking for a Sensitive Artificial Listening Agent

49 Andreea Niculescu (UT) Conversational interfaces for task-oriented spoken dialogues: design aspects influencing interaction quality

\section{2}

I Terry Kakeeto (UvT) Relationship Marketing for SMEs in Uganda

2 Muhammad Umair (VU) Adaptivity, emotion, and Rationality in Human and Ambient Agent Models

3 Adam Vanya (VU) Supporting Architecture Evolution by Mining Software Repositories

4 Jurriaan Souer (UU) Development of Content Management System-based Web Applications

5 Marijn Plomp (UU) Maturing Interorganisational Information Systems

6 Wolfgang Reinhardt (OU) Awareness Support for Knowledge Workers in Research Networks

7 Rianne van Lambalgen (VU) When the Going Gets Tough: Exploring Agent-based Models of Human Performance under Demanding Conditions

8 Gerben de Vries (UVA) Kernel Methods for Vessel Trajectories

9 Ricardo Neisse (UT) Trust and Privacy Management Support for Context-Aware Service Platforms

Io David Smits (TUE) Towards a Generic Distributed Adaptive Hypermedia Environment

II J.C.B. Rantham Prabhakara (TUE) Process Mining in the Large: Preprocessing, Discovery, and Diagnostics

I2 Kees van der Sluijs (TUE) Model Driven Design and Data Integration in Semantic Web Information Systems

I3 Suleman Shahid (UvT) Fun and Face: Exploring non-verbal expressions of emotion during playful interactions

I4 Evgeny Knutov (TUE) Generic Adaptation Framework for Unifying Adaptive Web-based Systems
I5 Natalie van der Wal (VU) Social Agents. AgentBased Modelling of Integrated Internal and Social Dynamics of Cognitive and Affective Processes.

I6 Fiemke Both (VU) Helping people by understanding them - Ambient Agents supporting task execution and depression treatment

I7 Amal Elgammal (UvT) Towards a Comprehensive Framework for Business Process Compliance

I8 Eltjo Poort (VU) Improving Solution Architecting Practices

I9 Helen Schonenberg (TUE) What's Next? Operational Support for Business Process Execution

20 Ali Bahramisharif (RUN) Covert Visual Spatial Attention, a Robust Paradigm for Brain-Computer Interfacing

2I Roberto Cornacchia (TUD) Querying Sparse Matrices for Information Retrieval

22 Thijs Vis (UvT) Intelligence, politie en veiligheidsdienst: verenigbare grootheden?

23 Christian Muehl (UT) Toward Affective BrainComputer Interfaces: Exploring the Neurophysiology of Affect during Human Media Interaction

24 Laurens van der Werff (UT) Evaluation of Noisy Transcripts for Spoken Document Retrieval

25 Silja Eckartz (UT) Managing the Business Case Development in Inter-Organizational IT Projects: A Methodology and its Application

26 Emile de Maat (UVA) Making Sense of Legal Text

27 Hayrettin Gurkok (UT) Mind the Sheep! User Experience Evaluation \& Brain-Computer Interface Games

28 Nancy Pascall (UvT) Engendering Technology Empowering Women

29 Almer Tigelaar (UT) Peer-to-Peer Information Retrieval

30 Alina Pommeranz (TUD) Designing HumanCentered Systems for Reflective Decision Making

3I Emily Bagarukayo (RUN) A Learning by Construction Approach for Higher Order Cognitive Skills Improvement, Building Capacity and Infrastructure

32 Wietske Visser (TUD) Qualitative multi-criteria preference representation and reasoning

33 Rory Sie (OUN) Coalitions in Cooperation Networks (COCOON) 
34 Pavol Jancura (RUN) Evolutionary analysis in PPI networks and applications

35 Evert Haasdijk (VU) Never Too Old To Learn - Online Evolution of Controllers in Swarm- and Modular Robotics

36 Denis Ssebugwawo(RUN) Analysis and Evaluation of Collaborative Modeling Processes

37 Agnes Nakakawa (RUN) A Collaboration Process for Enterprise Architecture Creation

38 Selmar Smit (VU) Parameter Tuning and Scientific Testing in Evolutionary Algorithms

39 Hassan Fatemi (UT) Risk-aware design of value and coordination networks

40 Agus Gunawan (UvT) Information Access for SMEs in Indonesia

4I Sebastian Kelle (OU) Game Design Patterns for Learning

42 Dominique Verpoorten (OU) Reflection Amplifiers in self-regulated Learning

43 (Withdrawn)

44 Anna Tordai (VU) On Combining Alignment Techniques

45 Benedikt Kratz (UvT) A Model and Language for Business-aware Transactions

46 Simon Carter (UVA) Exploration and Exploitation of Multilingual Data for Statistical Machine Translation

47 Manos Tsagkias (UVA) A Model and Language for Business-aware Transactions

48 Jorn Bakker (TUE) Handling Abrupt Changes in Evolving Time-series Data

49 Michael Kaisers (UM) Learning against Learning Evolutionary dynamics of reinforcement learning algorithms in strategic interactions

50 Steven van Kervel (TUD) Ontologogy driven Enterprise Information Systems Engineering

5I Jeroen de Jong (TUD) Heuristics in Dynamic Sceduling; a practical framework with a case study in elevator dispatching
2013

I Viorel Milea (EUR) News Analytics for Financial Decision Support

2 Erietta Liarou (CWI) MonetDB/DataCell: Leveraging the Column-store Database Technology for Effcient and Scalable Stream Processing

3 Szymon Klarman (VU) Reasoning with Contexts in Description Logics

4 Chetan Yadati (TUD) Coordinating autonomous planning and scheduling

5 Dulce Pumareja (UT) Groupware Requirements Evolutions Patterns

6 Romulo Gonzalves (CWI) The Data Cyclotron: Juggling Data and Queries for a Data Warehouse Audience

7 Giel van Lankveld (UT) Quantifying Individual Player Differences

8 Robbert-Jan Merk (VU) Making Enemies: Cognitive Modeling for Opponent Agents in Fighter Pilot Simulators

9 Fabio Gori (RUN) Metagenomic Data Analysis: Computational Methods and Applications

Io Jeewanie Jayasinghe Arachchige (UvT) A Unified Modeling Framework for Service Design

II Evangelos Pournaras (TUD) Multi-level Reconfigurable Self-organization in Overlay Services

I2 Maryam Razavian (VU) Knowledge-driven Migration to Services

I3 Mohammad Zafiri (UT) Service Tailoring: Usercentric creation of integrated IT-based homecare services to support independent living of elderly

I4 Jafar Tanha (UVA) Ensemble Approaches to SemiSupervised Learning Learning

I5 Daniel Hennes (UM) Multiagent Learning - Dynamic Games and Applications 


\section{Maastricht University}

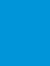

